\title{
Measuring Antineutrino Oscillations with the MINOS Experiment
}

\author{
Justin J. Evans
}

Jesus College, Oxford
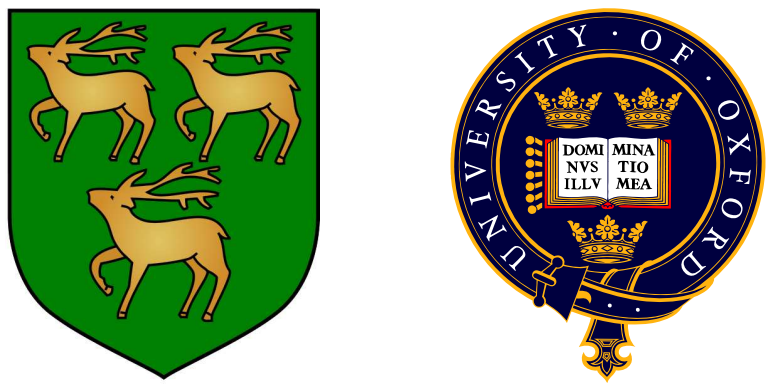

Thesis submitted in partial fulfilment of the requirements for the degree of Doctor of Philosophy at the University of Oxford

Trinity Term, 2008 


\title{
Measuring Antineutrino Oscillations with the MINOS Experiment
}

\author{
Justin J. Evans \\ Jesus College, Oxford \\ Thesis submitted in partial fulfilment of the requirements for the degree of \\ Doctor of Philosophy at the University of Oxford \\ Trinity Term, 2008
}

\begin{abstract}
MINOS is a long baseline neutrino oscillation experiment. A manmade beam of predominantly muon neutrinos is detected both $1 \mathrm{~km}$ and $735 \mathrm{~km}$ from the production point by two functionally identical detectors. A comparison of the energy spectra measured by the two detectors shows the energy-dependent disappearance of muon neutrinos characteristic of oscillations and allows a measurement of the parameters governing the oscillations. This thesis presents work leading to measurements of disappearance in the $6 \% \bar{\nu}_{\mu}$ background in that beam.

A calibration is developed to correct for time-dependent changes in the responses of both detectors, reducing the corresponding uncertainty on hadronic energy measurements from $1.8 \%$ to $0.4 \%$ in the near detector and from $0.8 \%$ to $0.4 \%$ in the far detector. A method of selecting charged current $\bar{\nu}_{\mu}$ events is developed, with purities (efficiencies) of $96.5 \%$ (74.4\%) at the near detector, and $98.8 \%$ $(70.9 \%)$ at the far detector in the region below $10 \mathrm{GeV}$ reconstructed antineutrino energy. A method of using the measured near detector neutrino energy spectrum to predict that expected at the far detector is discussed, and developed for use in the $\bar{\nu}_{\mu}$ analysis. Sources of systematic uncertainty contributing to the oscillation measurements are discussed.

In the far detector, 32 charged current $\bar{\nu}_{\mu}$ events are observed below a reconstructed energy of $30 \mathrm{GeV}$, compared to an expectation of 47.8 for $\Delta \bar{m}_{\text {atm }}^{2}=$ $\Delta m_{\text {atm }}^{2}, \sin ^{2}\left(2 \bar{\theta}_{23}\right)=\sin ^{2}\left(2 \theta_{23}\right)$. This deficit, in such a low-statistics sample, makes the result difficult to interpret in the context of an oscillation parameter measurement. Possible sources for the discrepancy are discussed, concluding that considerably more data are required for a definitive solution. Running MINOS with a dedicated $\bar{\nu}_{\mu}$ beam would be the ideal continuation of this work.
\end{abstract}




\section{Acknowledgements}

When a piece of work takes four years to complete, far too many people play parts in its development to be thanked individually. However, certain people deserve specific mention for contributions without which the completion of this thesis would have been all the more difficult.

First and foremost thanks go to my supervisor Giles Barr who guided me superbly through the process of learning to be a particle physics researcher. His perennially encouraging supervision and infectious enthusiasm for the subject benefited me hugely.

The many members of the Oxford MINOS group who passed through during my time all deserve thanks. Alfons Weber could always be relied on to provide some insightful comments on any histogram. Nathaniel Tagg supervised me through my early work in the MINOS calibration group, his in-depth knowledge in that area proving invaluable. Jeff Hartnell was one of the driving forces behind the antineutrino analysis which forms the main part of this thesis, and our numerous conversations helped crystallise many of the ideas I present.

I also shared my time at Oxford with Chris Backhouse, Anatael Cabrera, Katarzyna Grzelak, Phillip Litchfield, Paul Miyagawa, Robert Pittam, Tobias Raufer, Philip Rodrigues, Alex Sousa, Gemma Tinti and Nick West, all of whom were ready to answer my many questions, suggest new ideas, and engage in some fascinating discussions over coffee.

Thanks go to the many members of the MINOS collaboration who made my time both productive and enjoyable. Particular thanks go to the members of the antineutrino working group for their hard work in making the analysis happen.

Finally thanks go to may family, particularly to my parents, for their constant support over the last four years. 


\section{Contents}

1 Introduction 1

2 The History and Theory of Neutrino Physics 4

2.1 The Need for the Neutrino . . . . . . . . . . . . . . . . 5

2.2 The Neutrino Gains its own Identity . . . . . . . . . . . . . . . 6

2.3 The Discovery of the Neutrino . . . . . . . . . . . . . . . . . . 7

2.4 Parity Violation . . . . . . . . . . . . . . . . . . . . 8

2.5 The Weak Interaction . . . . . . . . . . . . . . . . . . . 10

2.6 Three Types of Neutrino . . . . . . . . . . . . . . . . . . . . . 11

2.7 Neutrino Flavour Change and Mass . . . . . . . . . . . . . . . . 13

2.7.1 Neutrino Oscillations . . . . . . . . . . . . . 13

2.7.2 The Two-Flavour Approximation . . . . . . . . . . . 17

2.8 Evidence for Neutrino Oscillations . . . . . . . . . . . . . . . . . . 19

2.8.1 Solar Neutrinos . . . . . . . . . . . . . . . . . 19

2.8.2 Atmospheric Neutrinos . . . . . . . . . . . . . . . . . . 29

$2.8 .3 \theta_{13} \ldots \ldots \ldots \ldots \ldots$

$2.9 \mathcal{C P}$ Violation . . . . . . . . . . . . . . . . . . . 38

$2.9 .1 \mathcal{C}$ Violation . . . . . . . . . . . . . . . . . 38

$2.9 .2 \quad \mathcal{C P}$ Conjugation . . . . . . . . . . . . . . . . . . 39

2.9.3 The Discovery of $\mathcal{C} \mathcal{P}$ Violation . . . . . . . . . . . . . . . 39

$2.10 \mathcal{C P} \mathcal{T}$ Invariance . . . . . . . . . . . . . . . . . . . . . 40 
$2.11 \mathcal{T}$ Invariance . . . . . . . . . . . . . . . . . . . . . . 40

$2.12 \mathcal{C P}$ Violation in the Neutrino Sector . . . . . . . . . . . . . . . . 41

$2.13 \mathcal{C P} \mathcal{T}$ Violation in the Neutrino Sector . . . . . . . . . . . . . 42

2.14 Looking for $\mathcal{C P} \mathcal{T}$ Violation with MINOS . . . . . . . . 45

2.15 Sterile Neutrinos . . . . . . . . . . . . . . . . 45

2.16 Absolute Neutrino Mass . . . . . . . . . . . . . . . . . 47

2.17 Majorana or Dirac Neutrinos? . . . . . . . . . . . . . . . . . . 48

$2.18 \nu_{\mu} \rightarrow \bar{\nu}_{\mu}$ Transitions in MINOS . . . . . . . . . . 48

3 The MINOS Experiment $\quad 50$

3.1 Neutrino Physics with MINOS . . . . . . . . . . . . . 50

$3.1 .1 \nu_{\mu}$ Disappearance . . . . . . . . . . . 50

3.1.2 A Two-Detector Experiment . . . . . . . . . . . . . . 52

3.1.3 Further Physics Potential of MINOS . . . . . . . . . . . 52

3.2 The NuMI Neutrino Beam . . . . . . . . . . . . . . . . . . 53

3.3 The MINOS Detectors . . . . . . . . . . . . . 56

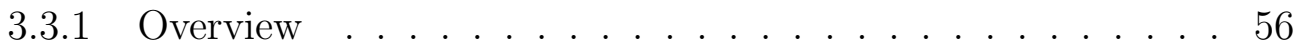

$3.3 .2 \quad$ Steel . . . . . . . . . . . . . . . . . . . . 59

3.3.3 Scintillator . . . . . . . . . . . . . . . . . . . 59

3.3.4 Photomultiplier Tubes . . . . . . . . . . . . . . . 62

3.3.5 Electronics . . . . . . . . . . . . . . . . . . 62

3.4 Light Injection $\ldots \ldots \ldots \ldots$

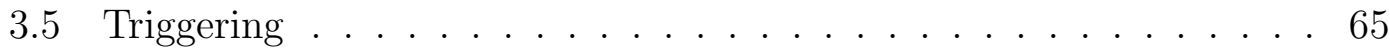

3.6 The Calibration Detector . . . . . . . . . . . . . . . . . 66

3.7 Simulation Software . . . . . . . . . . . . . . . . . 67

3.7 .1 Neutrino Beam . . . . . . . . . . . . . . . . . . . 67

3.7 .2 Detectors . . . . . . . . . . . . . . . . 68

3.8 Neutrino Interactions in the MINOS Detectors . . . . . . . . . 69 
3.8.1 Event Topologies . . . . . . . . . . . . . 69

3.8.2 Interaction Processes and Cross Sections . . . . . . . . . 72

3.8.3 Reconstruction Software . . . . . . . . . . . 75

3.8.4 Neutrino Energy Reconstruction . . . . . . . . . . 76

3.9 Data from the NuMI Beam . . . . . . . . . . . . . . 77

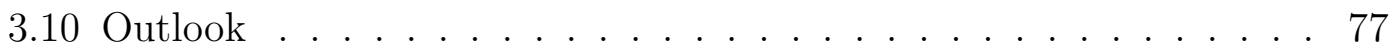

4 Drift Calibration $\quad 79$

4.1 Shower Energy Calibration . . . . . . . . . . . . . . 79

4.2 The Calibration Chain . . . . . . . . . . . . . . . . 81

4.3 The Need for a Drift Calibration . . . . . . . . . . . . . . 83

4.4 Drift Calibration Technique . . . . . . . . . . . . . . . 84

4.4 .1 Cosmic Muons . . . . . . . . . . . . . . . . 84

4.4.2 Path Length Correction . . . . . . . . . . . . . 88

4.4 .3 Hit Positions . . . . . . . . . . . . . . . . . . . . 92

4.4.4 Zero-Plane Reconstruction . . . . . . . . . . . . . . 92

4.4.5 Track Selection . . . . . . . . . . . . . . . 94

4.4.6 Plane Selection . . . . . . . . . . . . . . . . . 95

4.4.7 Tracking Detector Drift . . . . . . . . . . . . 95

4.4 .8 Granularity in Time . . . . . . . . . . . . . 96

4.4 .9 Version Shear . . . . . . . . . . . . . . . . . . 101

4.5 Results of the Drift Calibration . . . . . . . . . . . . . 103

4.5.1 Detector Response ... . . . . . . . . . . 103

4.5.2 Drift Constants . . . . . . . . . . . . 106

4.5.3 Estimating Temperature Dependence in the Far Detector . . 106

4.5.4 Effect on a Physics Analysis . . . . . . . . . . . 108

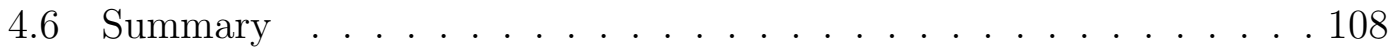


5 Muon Charge Identification

5.1 Introduction . . . . . . . . . . . . . . . . . 111

5.2 Reconstructed Charge .................... 112

5.3 Majority Curvature ...................... 115

5.4 Track Jitter . . . . . . . . . . . . . . . . . . . . . . . . 118

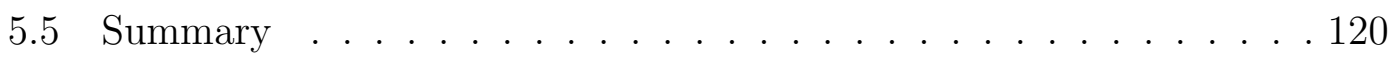

6 Charged Current $\bar{\nu}_{\mu}$ Event Selection $\quad 122$

6.1 Introduction . . . . . . . . . . . . . . . . . . . 122

6.2 Detector Noise . . . . . . . . . . . . . . . . . 123

6.3 Selection in the Near and Far Detectors . . . . . . . . . . . . . 123

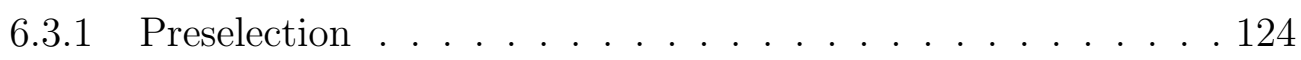

6.3.2 Muon Charge Identification . . . . . . . . . . . . . 125

6.3.3 Neutral Current Background Removal . . . . . . . . . 125

6.3.4 Wrong-Sign Charged Current Background Removal . . . . . 127

6.3.5 Overall Selection ................... 132

6.4 Agreements Between Distributions in the Data and Simulation . . . 134

6.5 Neutrino Event Selection . . . . . . . . . . . . . . . . . . 141

6.6 Summary . . . . . . . . . . . . . . . . . . . . 142

7 Antineutrino Extrapolation $\quad 144$

7.1 Inter-Detector Flux Differences . . . . . . . . . . . . . . 144

7.2 Extrapolation Methods . . . . . . . . . . . . . 146

7.3 Beam Matrix Extrapolation . . . . . . . . . . . . . . . . 147

7.3.1 Near Detector Purity Correction . . . . . . . . . . . . 150

7.3.2 Near Detector Reconstructed to True Energy Matrix . . . . 151

7.3.3 Near Detector Efficiency Correction . . . . . . . . . . . . 153

7.3.4 Calculating the Near Detector Flux . . . . . . . . . . . 153

7.3.5 Calculating the Beam Matrix ............. 154 
7.3.6 Using the Beam Matrix . . . . . . . . . . . . . 163

7.3.7 Far Detector Transformations . . . . . . . . . . . . . . 164

7.4 Cross-Checking the Extrapolation . . . . . . . . . . . . . 173

7.5 Far Detector Fitting . . . . . . . . . . . . . . . . 175

7.6 Extrapolation of Near Detector Data . . . . . . . . . . . 175

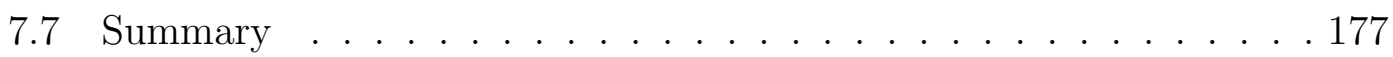

8 Systematic Uncertainties $\quad 179$

8.1 Introduction . . . . . . . . . . . . . . . . . . 179

8.2 Hadronic Energy Measurement . . . . . . . . . . . . . . 179

8.3 Muon Energy Measurement . . . . . . . . . . . . . . . . 181

8.4 Relative Near to Far Detector Spectrum Normalisation . . . . . . . 182

8.5 Flux Uncertainties . . . . . . . . . . . . . . . . . 183

8.5.1 General Beam Modelling . . . . . . . . . . . . . . 183

8.5.2 Antineutrinos from Decay Pipe Parents . . . . . . . . . . . . 184

8.6 Cross Section Uncertainties . . . . . . . . . . . . 186

8.7 Neutral Current Background . . . . . . . . . . . . . . . . . . 189

8.8 Charge Selection . . . . . . . . . . . . . . . . . . 189

8.8.1 Multiple Scattering . . . . . . . . . . . . . 190

8.8.2 Alignment of Detector Planes . . . . . . . . . . . . . 193

8.8.3 Unforeseen Charge Selection Uncertainties . . . . . . . . . . 194

8.9 Effect of Systematic Uncertainties on the Oscillation Analysis . . . 196

8.10 Effect of Systematic Uncertainties on the $\nu_{\mu} \rightarrow \bar{\nu}_{\mu}$ Transition Analysis202

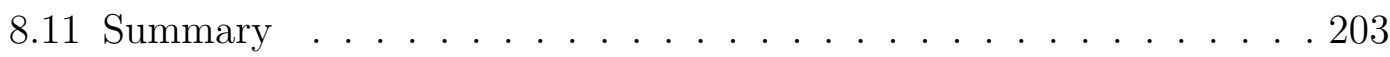

9 Charged Current $\bar{\nu}_{\mu}$ Analysis $\quad 204$

9.1 Introduction . . . . . . . . . . . . . . . . . . 204

9.2 Far Detector Data . . . . . . . . . . . . . . . . 205

9.3 Oscillation Analysis . . . . . . . . . . . . . . . . . 207 
9.3.1 Reducing the Width of the Energy Bins . . . . . . . . . . 210

9.4 Single Parameter Fit to $\Delta \bar{m}_{\text {atm }}^{2} \ldots \ldots$. . . . . . . . . . 210

$9.5 \bar{\nu}_{\mu}$ Appearance . . . . . . . . . . . . . . . . . . . 212

9.6 Summary . . . . . . . . . . . . . . . . . . . 213

10 Discussion $\quad 214$

10.1 Summary . . . . . . . . . . . . . . . . . . . 214

10.2 Antineutrino Analysis . . . . . . . . . . . . . . . . 215

10.3 Outlook . . . . . . . . . . . . . . . . . 220

10.4 Conclusion . . . . . . . . . . . . . . . . . . . 222

$\begin{array}{ll}\text { Bibliography } & 224\end{array}$ 


\section{List of Figures}

2.1 The possible interactions of a neutrino. . . . . . . . . . . 11

2.2 The flux of $\nu_{e}$ and $\nu_{\mu}+\nu_{\tau}$ from the Sun measured with the SNO

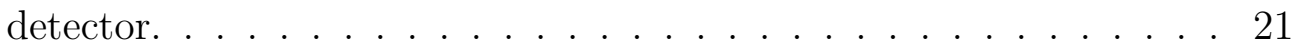

2.3 A summary of the constraints on neutrino oscillation parameters afforded by all data to date. . . . . . . . . . . . . . . 23

2.4 The most recent results of the KamLAND experiment. . . . . . . . 29

2.5 Zenith angle atmospheric neutrino distributions measured by the Super-Kamiokande detector. . . . . . . . . . . . . . . . . 32

2.6 The deficit in atmospheric neutrino events measured by the SuperKamiokande detector as a function of $L / E \ldots \ldots \ldots 33$

2.7 The regions in atmospheric neutrino oscillation parameter space allowed by the most accurate experiments. . . . . . . . . . . 35

2.8 Projected sensitivities for various MINOS exposures. . . . . . . . . 35

2.9 The predicted sensitivity of MINOS to the mixing angle $\theta_{13} \ldots \ldots 38$

2.10 The limits on a difference between $\Delta m_{\text {atm }}^{2}$ and $\Delta \bar{m}_{\text {atm }}^{2}$ set by analysis of atmospheric neutrinos in the MINOS far detector. . . . . . . 44

2.11 The combined world limits on differences between $\Delta m_{\mathrm{atm}}^{2}$ and $\Delta \bar{m}_{\mathrm{atm}}^{2} .44$

2.12 The spectrum of neutral current events observed at the MINOS far

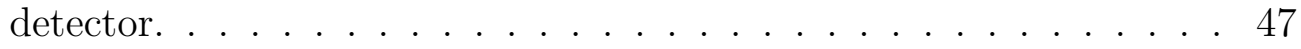

3.1 The most recently published MINOS $\nu_{\mu}$ disappearance measurement. 51 
3.2 The $\nu_{\mu}$ survival probability in the two-flavour model as a function of neutrino energy. . . . . . . . . . . . . . . . . 52

3.3 The components of the NuMI beamline. . . . . . . . . . . . . . 54

3.4 Three possible configurations of the NuMI beam. . . . . . . . . . . 55

3.5 The spectra of CC neutrino interactions in the near detector in the LE-10 beam configuration arising from the various neutrino flavours in the beam. . . . . . . . . . . . . . . 56 56

3.6 The MINOS near and far detectors. . . . . . . . . . . . . . . 57

3.7 A schematic of a MINOS detector. . . . . . . . . . . . . 57

3.8 A near detector plane, showing the partially and fully instrumented regions. . . . . . . . . . . . . . . . . 59

3.9 The structure of a scintillator strip. . . . . . . . . . . . . . 60

3.10 The scintillator module arrangement on a near detector plane. . . . 60

3.11 The scintillator module arrangement on a far detector plane. . . . . 61

3.12 The calorimetric energy resolution of the MINOS detectors to hadronic and electromagnetic showers, as measured by the calibration detector. 67

3.13 The charged current interaction of a $\nu_{\mu}$ with an iron nucleus. . . . . 69

3.14 A typical high energy charged current $\nu_{\mu}$ event in the MINOS simulation. . . . . . . . . . . . . . . . . . . 69

3.15 A typical low energy charged current $\nu_{\mu}$ event in the MINOS simulation. . . . . . . . . . . . . . . . . 70

3.16 A high energy charged current $\nu_{\mu}$ event in the MINOS simulation in which the $\mu^{-}$undergoes a hard scatter. . . . . . . . . . 71

3.17 The neutral current interaction of a neutrino with an iron nucleus. . 71

3.18 A typical neutral current event in the MINOS simulation. . . . . . . 72

3.19 A neutral current event in the MINOS simulation in which a track has been reconstructed across the hadronic shower. . . . . . . . . 73 
3.20 A neutral current event in the MINOS simulation which contains a true track from a hadronic particle. . . . . . . . . . . . 73

3.21 A charged current $\nu_{e}$ event in the MINOS simulation. . . . . . . . . 74

3.22 The data taken by the MINOS detectors up to June 2007. . . . . . 77

4.1 The calibration chain. . . . . . . . . . . . . . . 81

4.2 A typical far detector cosmic muon. . . . . . . . . . . . . . . . . 89

4.3 The stopping power of muons in copper. . . . . . . . . . . . . . . 89

4.4 The energy loss per plane of muons in scintillator. . . . . . . . . . . 90

4.5 Plane-summed pulse height against path length correction factor before and after the path length correction. . . . . . . . . . 91

4.6 The reconstructed pulse-height weighted mean positions of zeroplanes in both detectors. . . . . . . . . . . . . . . 93

4.7 An illustration of how a reconstruction failure can result in detector activity being assigned a position outside the physical detector. . . 94

4.8 The distribution of selected plane-summed pulse heights over a 24hour period in both detectors. . . . . . . . . . . . . 96

4.9 The mean, median, and a Gaussian fit to the peak of the near detector response over a six-month period, and the corresponding detector hall temperature. . . . . . . . . . . . . . . . 97

4.10 The mean, median, and a Gaussian fit to the peak of the far detector response over a six-month period, and the corresponding detector hall temperature. . . . . . . . . . . . . . . . . . . . 98

4.11 The median near detector response for 24-, 8- and 3-hour data periods, superimposed with near detector hall temperatures. . . . . . 99

4.12 The median far detector response for 24-, 8- and 3-hour data periods, superimposed with far detector hall temperatures. . . . . . . 100 
4.13 The mean near detector response for 3-hour time periods, from which has been subtracted the corresponding 24-hour median response. . . . . . . . . . . . . . . . . . . 101

4.14 Near detector response and hall temperature over the full detector lifetime. . . . . . . . . . . . . . . . . . 104

4.15 Far detector response and hall temperature over the full detector lifetime. . . . . . . . . . . . . . . . . 105

4.16 The median plane-summed response of the far detector, corrected according to the temperature of the detector hall. . . . . . . . 107

4.17 The drift constants applicable to the beam data for both detectors, calculated using the median and mean detector response. . . . . . . 109

5.1 The reconstructed charge divided by momentum of tracks passing the preselection. . . . . . . . . . . . . . . . 112

5.2 Events failing and passing the track fit. . . . . . . . . . . . . . 113

5.3 Energy spectra of events attributed a positive charge by the reconstruction. . . . . . . . . . . . . . . . . 114

5.4 The calculation of majority curvature . . . . . . . . . . . . 116

5.5 A wrong-sign kink in a track caused by a change in sign of $x$ or $y$ co-ordinates. . . . . . . . . . . . . . . . . 117

5.6 Majority curvature of events attributed a positive charge by the

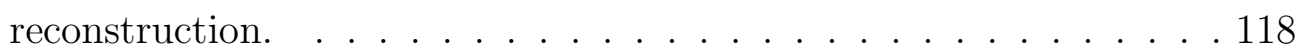

5.7 The fraction of events, as a function of track length, which, having been attributed positive charge by the reconstruction, have negative majority curvature. . . . . . . . . . . . . . . . . . 119

5.8 Track jitter for events with a positive reconstructed charge and majority curvature. . . . . . . . . . . . . . 120 
6.1 The neutral current PID for events with a positive reconstructed charge and majority curvature. . . . . . . . . . . . . 126

6.2 The neutral current PID as a function of reconstructed neutrino energy for events with a positive reconstructed charge and majority curvature. . . . . . . . . . . . . . . . . . . . . 128

6.3 Track jitter as a function of the neutral current PID for events with a positive reconstructed charge and majority curvature. . . . . . . . 129

6.4 Track fit uncertainty for tracks with positive reconstructed charge and majority curvature, passing the neutral current removal. . . . . 130

6.5 Track fit probability for tracks with positive reconstructed charge and majority curvature, passing the neutral current removal and track fit uncertainty cuts. . . . . . . . . . . . . . . 131

6.6 The track length of events passing all other selection cuts. . . . . . 132

6.7 Energy spectra and efficiency and purity graphs of the complete antineutrino selection. . . . . . . . . . . . . . 133

6.8 The near detector data and Monte Carlo distributions of $\frac{q}{p}$ and $C_{M}$ after making only the preselection cuts. . . . . . . . . . . 135

6.9 The near detector data and Monte Carlo distributions of $\frac{q}{p}$ and $C_{M}$ after making all other cuts. . . . . . . . . . . . . . 136

6.10 The near detector data and Monte Carlo distributions of $\sigma\left(\frac{q}{p}\right)$ after making all other cuts. . . . . . . . . . . . . 137

6.11 The near detector data and Monte Carlo distributions of $P_{D}$ and $J$ after making all other cuts. . . . . . . . . . . . . . . 138

6.12 The near detector data and Monte Carlo distributions of $P_{\text {fit }}$ after making all other cuts. . . . . . . . . . . . . . . . . . . . 139

6.13 The near detector data and Monte Carlo distributions of track length after making all other cuts. . . . . . . . . . . . . 140 
6.14 The near detector data and Monte Carlo distributions of reconstructed neutrino energy after making all selection cuts. . . . . . . . 140

6.15 The charged current $\nu_{\mu}$ efficiency and purity achieved by the $k$ nearest-neighbour selection technique. . . . . . . . . . . . . 142

7.1 An illustration of the different solid angles subtended by the near and far detectors at the neutrino parent decay point. . . . . . . 145

7.2 A simulation of the different neutrino energy spectra produced by the same neutrino parents in the near and far detectors. . . . . . . . 145

7.3 The steps involved in the beam matrix extrapolation of the MINOS neutrino oscillation analysis. . . . . . . . . . . . . 147

7.4 The steps involved in the beam matrix extrapolation developed for the analysis of antineutrinos in this thesis. . . . . . . . . . . 149

7.5 The purity correction applied in the near detector. . . . . . . . . . 151

7.6 The reconstructed to true energy conversion matrices applied at the near detector. . . . . . . . . . . . . . . . . . 152

7.7 The near detector efficiency correction. . . . . . . . . . . . . 153

7.8 A cartoon demonstrating how the far detector neutrino flux produced by each partial parent flux is filled into the beam matrix . . . 161

7.9 The beam matrices used to convert near detector neutrino and antineutrino fluxes into far detector fluxes. . . . . . . . . . . . 164

7.10 The far detector efficiency correction. . . . . . . . . . . . 166

7.11 The efficiency for neutrino and antineutrino events to be wrongly reconstructed and selected as antineutrino or neutrino events at the far detector. . . . . . . . . . . . . . . . . . 167

7.12 The true to reconstructed energy conversion matrices applied at the far detector. . . . . . . . . . . . . . . . . . 168 
7.13 The far detector purity correction, relative to an unoscillated energy spectrum. . . . . . . . . . . . . . . . . 168

7.14 The far detector neutral current contamination fraction, relative to an unoscillated energy spectrum. . . . . . . . . . . . . 169

7.15 The true to reconstructed energy conversion matrices for the wrongsign charged current background applied at the far detector. . . . . 171

7.16 The far detector $\stackrel{(-)}{\nu}_{\tau}$ efficiency correction. . . . . . . . . . . . . . . 172

7.17 The true to reconstructed energy conversion matrices applied to the $\nu_{\tau}$ and $\bar{\nu}_{\tau}$ predicted spectra. . . . . . . . . . . . . 173

7.18 The far detector Monte Carlo compared to the prediction obtained by applying the beam matrix extrapolation to the near detector Monte Carlo. . . . . . . . . . . . . . . . . . . . . . . . . . 174

7.19 The far detector predictions obtained through extrapolating the near detector data. . . . . . . . . . . . . . . . 176

7.20 The CC $\bar{\nu}_{\mu}$ far detector prediction obtained through extrapolating the near detector data, on a logarithmic $y$-axis scale in order to show the predicted background contributions. . . . . . . . . 177

8.1 The absolute hadronic energy uncertainty. . . . . . . . . . . . 181

8.2 The error band on the $\bar{\nu}_{\mu}$ reconstructed energy spectrum at the near detector given by the beam reweighting parameters. . . . . . . 183

8.3 A comparison of the near detector $\bar{\nu}_{\mu}$ spectrum in the standard NuMI beam configuration to that with the magnetic focusing horns switched off. . . . . . . . . . . . . . . . . . 185

8.4 The changes in the selected near detector $\bar{\nu}_{\mu}$ spectrum corresponding to the systematic uncertainties in the quasi-elastic and resonance axial masses. . . . . . . . . . . . . . . . . . . 187 
8.5 The changes in the selected near detector $\bar{\nu}_{\mu}$ spectrum corresponding to the $8 \%$ systematic uncertainties in the charged current $\bar{\nu}_{\mu}$ quasi-elastic and resonance cross sections. . . . . . . . . . 188

8.6 The changes in the selected near detector $\bar{\nu}_{\mu}$ spectrum corresponding to the systematic uncertainties in the parameters governing the cross section in the DIS-resonance transition region. . . . . . . . . 188

8.7 A comparison of the angle of bending induced in a single MINOS iron plane from the magnetic field and from multiple scattering, as a function of muon energy. . . . . . . . . . . . . . . . . . 191

8.8 The ratio of the total bending of a track due to magnetic field to that from multiple scattering, for a range of track lengths as a function of initial muon energy. . . . . . . . . . . . . . . . . . 192

8.9 The track energy from curvature for contained events with a track energy measured from range of $<5 \mathrm{GeV}$. . . . . . . . . . . 195

8.10 A graphical representation of the systematic shifts for input oscillation parameters of $\Delta \bar{m}_{\mathrm{atm}}^{2}=2.5 \times 10^{-3} \mathrm{eV}^{2}, \sin ^{2}\left(2 \bar{\theta}_{23}\right)=1.0 \ldots .198$

8.11 A graphical representation of the systematic shifts for input oscillation parameters of $\Delta \bar{m}_{\mathrm{atm}}^{2}=6.0 \times 10^{-3} \mathrm{eV}^{2}, \sin ^{2}\left(2 \bar{\theta}_{23}\right)=1.0 \ldots .200$

8.12 The systematic error band on the $\bar{\nu}_{\mu}$ prediction. . . . . . . . . 203

9.1 The far detector $\mathrm{CC} \bar{\nu}_{\mu}$ data with the $\mathcal{C P} \mathcal{T}$-conserving prediction and systematic error band. . . . . . . . . . . . . . 205

9.2 The energy spectra of the data and predictions at a variety of $\Delta \bar{m}_{\text {atm }}^{2}$ values, shown with the underlying oscillation functions. . . . . . . 208

9.3 The full $\chi^{2}$ surface as a function of the $\bar{\nu}_{\mu}$ oscillation parameters, and the corresponding $68 \%, 90 \%$ and $99 \%$ confidence intervals relative to the global $\chi^{2}$ minimum. . . . . . . . . . . . . . 209 
9.4 The $68 \%, 90 \%$ and $99 \%$ antineutrino oscillation confidence intervals obtained using $0.5 \mathrm{GeV}$ energy bins. . . . . . . . . . . . . . 211

9.5 The $\chi^{2}$ as a function of $\Delta \bar{m}_{\text {atm }}^{2}$ with $\sin ^{2}\left(2 \bar{\theta}_{23}\right)$ fixed to 1.0. . . . . 211

10.1 The far detector data and Monte Carlo CC $\bar{\nu}_{\mu}$ spectra for two alternative selections. . . . . . . . . . . . . . . 218

10.2 The radial position (squared) of the reconstructed track start points for the $\nu_{\mu}$ and $\bar{\nu}_{\mu}$ events. . . . . . . . . . . . . . . . . . 219

10.3 The MINOS sensitivity to the antineutrino oscillation parameters with the $\bar{\nu}_{\mu}$ component in the neutrino beam, compared to a dedicated $\bar{\nu}_{\mu}$ beam. . . . . . . . . . . . . . . . . . . . . 221 


\section{List of Tables}

4.1 The periods of data calibrated using each different reconstruction software version. . . . . . . . . . . . . . . . 102

4.2 Detector response correction factors for differing reconstruction ver-

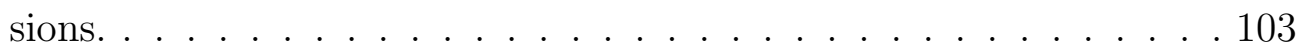

6.1 A summary of the charged current $\bar{\nu}_{\mu}$ selection cuts. . . . . . . . . 132

7.1 Symbols used in the explanation of the beam matrix extrapolation method. . . . . . . . . . . . . . . . . . 178

8.1 The shifts in the measured antineutrino oscillation parameters induced by sources of systematic uncertainty at the estimated $1 \sigma$ level, at input oscillation parameters of $\Delta \bar{m}_{\mathrm{atm}}^{2}=2.5 \times 10^{-3} \mathrm{eV}^{2}$, $\sin ^{2}\left(2 \bar{\theta}_{23}\right)=1.0 \ldots \ldots \ldots$. . . . . . . . . . . . . 197

8.2 The shifts in the measured antineutrino oscillation parameters induced by sources of systematic uncertainty at the estimated $1 \sigma$ level, at input oscillation parameters of $\Delta \bar{m}_{\mathrm{atm}}^{2}=2.5 \times 10^{-3} \mathrm{eV}^{2}$, $\sin ^{2}\left(2 \bar{\theta}_{23}\right)=1.0 \ldots \ldots \ldots$. . . . . . . . . . . . . . . . . . . . . . . . . .

8.3 The shifts in the measured antineutrino oscillation parameters induced by sources of systematic uncertainty at the estimated $1 \sigma$ level, at input oscillation parameters of $\Delta \bar{m}_{\text {atm }}^{2}=6.0 \times 10^{-3} \mathrm{eV}^{2}$, $\sin ^{2}\left(2 \bar{\theta}_{23}\right)=1.0 \ldots \ldots . \ldots \ldots 9$ 
8.4 The shifts in the measured antineutrino oscillation parameters induced by sources of systematic uncertainty at the estimated $1 \sigma$ level, at input oscillation parameters of $\Delta \bar{m}_{\mathrm{atm}}^{2}=6.0 \times 10^{-3} \mathrm{eV}^{2}$, $\sin ^{2}\left(2 \bar{\theta}_{23}\right)=1.0 \ldots \ldots \ldots \ldots$. . . . . . . . . . . . . . . . . . . . . .

9.1 Numbers of events observed and expected in the case of no oscillations in the far detector $\mathrm{CC} \bar{\nu}_{\mu}$ spectrum, along with the corresponding statistical deficit observed in the data. . . . . . . . . 206 


\section{Chapter 1}

\section{Introduction}

The neutrino has proved one of the hardest of fundamental particles to pin down. Despite being one of the most numerous particles in the universe it remained unknown until the 1930s, even then beginning life only as the most tentative of ideas: another twenty years had to pass before it would finally be directly observed.

The difficulties in experimental neutrino physics arise primarily from the incredible weakness with which the neutrino interacts with any other matter, an interaction strength governed by the aptly named weak interaction which itself was a concept born and developed in parallel with the neutrino. Yet despite these inherent challenges, the branch of particle physics that has grown around this area has yielded some of the most exciting and surprising results of the 20th century.

Through our investigations into the weak interaction we have been forced to give up some of our most strongly held beliefs about the underlying symmetries of the universe, one of the last standing of which will be investigated in this thesis. But the neutrino itself has commanded more than its fair share of physicists' attention as, when it was finally observed in detail, it exhibited the unexpected and exotic behaviour of oscillation between flavour states. This was in direct conflict with one of the basic assumptions of the standard model: that of flavour conservation; and it furthermore required the neutrino to have a hitherto unexpected 
non-zero mass, but a mass so much smaller than any other fermion that some more fundamental, as yet undiscovered, process is thought to be responsible.

It is to our knowledge of neutrino oscillations that this thesis contributes. It begins, in chapter 2, with an overview of the history of the neutrino from its inception in the 1930s through to the discovery and investigation of the oscillation phenomenon. Summarising the mathematical model of oscillations and the experimental work leading to our knowledge of the parameters involved, neutrino physics is seen to be moving into a period of precision measurement. The work in this thesis is based one such precision experiment: the MINOS experiment.

MINOS (the Main Injector Neutrino Oscillation Search), introduced in detail in chapter 3 , is a long-baseline experiment, producing a manmade beam of muon neutrinos from the NuMI (Neutrinos at the Main Injector) beam at Fermilab, and letting them propagate over a distance of $735 \mathrm{~km}$. Measuring the neutrino energy spectrum with a detector near the production point, and another after the full $735 \mathrm{~km}$, allows a precision measurement of the so-called atmospheric oscillation parameters.

Such high precision requires accurate measurement of neutrino energies, an important part of which is the measurement of hadronic shower energies. This is facilitated by a chain of calibrations, one of which is developed for this thesis in chapter 4: a method of using cosmic muons to track the change in response of the detectors over time.

The NuMI beam contains a $6 \%$ background of muon antineutrinos. The magnetised MINOS detectors allow separation of charged current $\nu_{\mu}$ and $\bar{\nu}_{\mu}$ events, allowing the first direct measurements of the atmospheric regime oscillation parameters for neutrinos and antineutrinos separately. Any deviation between the two would be evidence of $\mathcal{C P} \mathcal{T}$ violation. Additionally, it is possible to look for $\bar{\nu}_{\mu}$ appearance which, if observed, could indicate that the $\nu_{\mu}$ disappearance indicative of oscillations was happening through an exotic $\nu_{\mu} \rightarrow \bar{\nu}_{\mu}$ channel rather than the 
assumed (but not yet directly observed) $\nu_{\mu} \rightarrow \nu_{\tau}$ channel. In this thesis these antineutrino measurements are made.

A necessary ingredient to an analysis of the antineutrinos is the selection of charged current $\bar{\nu}_{\mu}$ events. Such a selection is developed for this thesis: two new muon charge-identification variables are developed in chapter 5, and these are used to form a complete selection method in chapter 6. A second ingredient is a method of extrapolating the measured energy spectrum from the near to far detectors. One such method (used in previous MINOS $\nu_{\mu}$ analyses) has, as explained in chapter 7 , been further developed to allow the simultaneous extrapolation of $\nu_{\mu}$ and $\bar{\nu}_{\mu}$ spectra, allowing for differing neutrino and antineutrino oscillation parameters. Chapter 8 presents the final ingredient necessary for the analysis: a summary of the sources of systematic uncertainty which affect the measurements.

Chapter 9 presents the results of the $\bar{\nu}_{\mu}$ analysis. Given the low statistics, the constraints on the oscillation parameters are correspondingly weaker than those for the neutrinos; but they represent the first direct measurement of these parameters separate from those of the neutrinos. A limit is also placed on $\bar{\nu}_{\mu}$ appearance.

Finally, chapter 10 summarises the results obtained in the context of our current understanding as has been discussed in chapter 2. It furthermore looks at the future directions the MINOS experiment, and the neutrino physics community at large, may look to go in light of what has been presented. 


\section{Chapter 2}

\section{The History and Theory of Neutrino Physics}

Almost all reviews of the history of neutrino physics begin with a reference to Wolfgang Pauli's letter of 1930 [1] in which a new, light, neutral, weakly interacting particle was first proposed. This is indeed a fitting place to begin as Pauli was the first person with the insight (and the status) to be able to unfashionably increase the complexity of the physicist's model of the world by introducing a seemingly unnecessary (and, worse still, undetectable) new particle.

Yet the 'neutronen' (or neutron) as Pauli called it was not the neutrino we know today: in trying to solve all the problems of atomic theory in one sweep, Pauli had actually predicted a hybrid of the neutron and neutrino. It took the discovery of the neutron by James Chadwick and the formulation of a theory of beta decay by Enrico Fermi for the neutrino to gain the beginnings of an identity that has continued to fascinate physicists for the next 70 years. 


\subsection{The Need for the Neutrino}

The first two decades of the 20th century saw a number of physicists addressing the postulate that the electron produced in the $\beta$-decay of an atom had a unique energy: not a continuous distribution. This proved less than straightforward as the usual spectrographic technique of bending the electrons through a magnetic field and measuring the amount of curvature by the position of arrival on a photographic plate was flawed by the propensity of the plate to show so strongly any lines in the spectrum (such as those from internal conversion) that any underlying continuous distribution was masked.

Two leading proponents of $\beta$-decay studies were Otto Hahn and Lise Meitner who, in 1908, reported that the $\beta$-decay electrons were produced with a unique energy [2]; but after further experimentation they obtained, in 1911, results showing an underlying continuous spectrum [3]. The continuous distribution of energies was confirmed in 1914 by Chadwick using a Geiger counter instead of a photographic plate [4].

Even a confirmation of the continuous spectrum of electron energies was not cause enough to warrant a new particle: the electrons may merely have been losing energy on the way out of the radioactive material (perhaps through collisions with other atoms). The definitive experiment was an ingenious piece of precision physics performed by Charles Ellis and William Wooster in 1927 [5]. They placed a radium source on a $1 \mathrm{~mm}$ diameter nickel wire in a slightly larger brass tube, inside a $3.5 \mathrm{~mm}$ diameter lead tube: enough to completely absorb all $\beta$-decay electrons emitted by the radium. By measuring the $\mathcal{O}\left(1 / 1000^{\circ} \mathrm{C}\right)$ temperature rise of the system, they could measure the total energy released in the $\beta$-decays. If the electrons were all emitted with the same energy but lost energy through collisions in the source medium, the energy measured would have been consistently that of the highest-energy $\beta$-decay electrons: $1.05 \mathrm{MeV}$. However, the average energy 
of the $\beta$-decay spectrum was measured, leaving no room for energy to have been deposited in the source. The electrons must have been emitted with a range of energies.

Niels Bohr's quantised model of the atom showed that $\beta$-decay electrons were of too high an energy to be those from the atomic orbitals. Therefore they must emanate from the nuclei, so must have a unique energy. The experimental evidence contradicting this theory caused Bohr to doubt the conservation of energy. Pauli, however, took a different tack in his famous letter: proposing a new, undetectable particle that could carry off the missing energy.

Pauli also refers to the then problem of the spins of nuclei such as Nitrogen. It was known that nuclei were too heavy to be made purely from protons. Instead, the popular model, taking as an example ${ }^{14} \mathrm{~N}$, was a nucleus containing seven protons to give the correct charge, seven more protons to supply the necessary mass, and seven electrons to cancel the charge of the extra protons. This totals an odd number of spin-half particles, which must give an overall half-integer spin. However, the ${ }^{14} \mathrm{~N}$ nucleus was measured to have integer spin by Franco Rasetti in $1929[6,7]$ (although it took Walter Heitler and Gerhard Herzberg to interpret his results as showing the Bose-Einstein nature for the nucleus [8]). Some number

of Pauli's neutronens, with spin $\frac{1}{2}$, could exist within the nucleus and provide the missing angular momentum.

\subsection{The Neutrino Gains its own Identity}

The emergence of the neutrino from the neutronen concept began with the discovery, in 1932, of the neutron by Chadwick [9]. He investigated radiation given off by beryllium and boron, at the time thought to consist of photons. He found it to be too penetrating to consist of photons. Furthermore, when passing through matter, the atoms ejected through collisions with the radiation were of too high 
an energy for the radiation to be photonic. Chadwick concluded the radiation consisted of neutral particles slightly heavier than the proton: neutrons. However, he considered these to be compound particles: a bound state of a proton and an electron. $^{1}$

In 1932 Dmitri Iwanenko discussed the concept of the neutron as a fundamental particle [10] and Werner Heisenberg developed this into a more detailed model of nuclear structure $[11,12,13]$. In 1934, Iwanenko suggested the $\beta$-decay electron could be created during the process, analogously to the creation of a photon in electromagnetic decays [14]. Around the same time, Francis Perrin [15] and Enrico Fermi [16] put forward the idea of the neutrino as a massless particle created during the process of $\beta$ decay. Fermi went into the greater mathematical detail (see [17] for an English translation of his paper), using perturbation theory to calculate a matrix element for the interaction at a single point of a neutron, proton, electron and neutrino. He was able to calculate the dependence on the neutrino mass of the energy spectrum of the emitted electron, allowing him to conclude the mass of the neutrino to be very small.

\subsection{The Discovery of the Neutrino}

It was not until the 1950s that the neutrino was directly observed. Attempts had been made $[18,19]$ but all had been thwarted by the very small cross section for neutrino interactions $\left(\sim 6 \times 10^{-20}\right.$ barn for $\bar{\nu} p$ interactions [20]).

The discovery was made in 1953 by Fred Reines and Clyde Cowan at the Hanford reactor site $[21,22,20,23]$. A tank of 300 litres of cadmium-doped liquid scintillator, surrounded by photomultiplier tubes (PMTs), was placed next to a reactor. An antineutrino would interact through $\bar{\nu} p \rightarrow n e^{+}$. The positron

\footnotetext{
${ }^{1}$ Chadwick makes the passing comment that viewing the neutron as an elementary particle has 'little to recommend it at present except the possibility of explaining the statistics of such nuclei as ${ }^{14} \mathrm{~N}^{\prime}$.
} 
annihilates with an electron in the tank producing two prompt photons (detected as a single pulse). The neutron is captured by the Cadmium to produced a delayed photon, required to be within $9 \mu$ s of the positron pulse. $2.55 \pm 0.15$ counts/minute were measured with the reactor on and $2.14 \pm 0.13$ counts/minute with the reactor off.

This $\sim 2 \sigma$ signal was promising but not conclusive, so Reines and Cowan performed a further experiment at the Savannah River Plant in 1956 [23, 24]. Three layers of scintillator were alternated with two layers of cadmium-doped water. The neutrino, interacting in the water, produced a positron almost at rest which decayed to two back-to-back photons. The neutron then produced a delayed photon when captured by the Cadmium. Simultaneous signals in two adjacent scintillator layers signified the positron photons, and the delayed photon from the neutron was required to be detected in one of these two layers. Any signal in the third scintillator layer implied the event may have been caused by a cosmic ray, thus acting as an effective veto. The number of counts with the reactor on minus the number with the reactor off was $1.23 \pm 0.24$ hour $^{-1}$ in the first running period and $0.93 \pm 0.22$ hour $^{-1}$ in the second (the two periods with slightly different detector setups). This much clearer signal demonstrated conclusively the direct observation of the neutrino.

\subsection{Parity Violation}

By 1956 the then named $\theta^{+}$and $\tau^{+}$particles had been discovered, decaying respectively into two and three pions (positive and negative parity states respectively $[25,26])$. Assuming conservation of parity, the $\theta^{+}$and $\tau^{+}$must have been two separate particles with different intrinsic parities. However, these particle had been measured to have the same lifetimes to within $10 \%[27,28,29]$ and the same masses to within 1\% [26]. This caused Tsung-Dao Lee and Chen-Ning Yang to 
investigate the experimental evidence for parity conservation [30]. They discovered there was good evidence for its conservation in strong and electromagnetic interactions, but not in weak interactions. This allowed them to suggest the $\theta^{+}$ and $\tau^{+}$were the same particle, decaying into two opposite parity states. Lee and Yang suggested a number of experiments to directly observe parity violation, one of which Chen-Shiung Wu performed the same year [31].

Wu placed a sample of ${ }^{60} \mathrm{Co}$, at low temperature, in a magnetic field so that the spins of the nuclei were aligned. The $\beta$-decay electrons were emitted preferentially in the direction opposite to the nuclear spin: direct evidence for parity violation (as upon parity reversal the electron momentum changes direction but the nuclear spin does not). The $\theta^{+}$and $\tau^{+}$could then be viewed as two decay channels of the same particle, now called the $K^{+}$.

An investigation of the particle spins and momenta involved in the $\mathrm{Wu}$ experiment suggests a preference of the antineutrino to take a positive helicity. However, without a measurement of the exact direction of the neutrino with respect to the nuclear spin, a direct measurement had not been made. A direct measurement of the helicity of the neutrino was made by Maurice Goldhaber, Lee Grozdins and Andrew Sunyar in 1957 [32]. A sample of ${ }^{152} \mathrm{Eu}$ (nuclear spin 0) was allowed to decay by $K$-electron capture to ${ }^{152} \mathrm{Sm}^{*}$ (nuclear spin 1 ). The spin of the emitted neutrino must be in the opposite direction to that of the ${ }^{152} \mathrm{Sm}^{*}$ nucleus (such that the total final state spin sums to $\frac{1}{2}$ : the spin of the initial-state electron). The ${ }^{152} \mathrm{Sm}^{*}$ is left with recoil momentum in the direction opposite to the neutrino and parallel or anti-parallel to its nuclear spin (depending on the neutrino helicity). ${ }^{152} \mathrm{Sm}^{*}$ emits a photon to decay to the spin-0 state ${ }^{152} \mathrm{Sm}$. The photons emitted parallel to the ${ }^{152} \mathrm{Sm}^{*}$ momentum take the nuclear spin away, thus having the same helicity as the neutrino. These photons, boosted slightly by the nuclear recoil momentum, have the correct energy to undergo resonant scattering by a further ${ }^{152} \mathrm{Sm}$ sample. Observing these resonant scatters therefore selects 
those photons which tag the neutrino helicity. The resonantly scattered photons were passed through magnetised iron, which could more easily absorb photons if their spin was aligned with the magnetic field, allowing a measurement of the photon helicity. The photon, and therefore the neutrinos, were measured to be left handed, at a level consistent with $100 \%$.

\subsection{The Weak Interaction}

Fermi $[16,17]$ developed his theory of the weak interaction by analogy with the electromagnetic interaction. He wrote the $\beta$-decay matrix element in the form $G\left(\bar{u}_{n} \gamma^{\mu} u_{p}\right)\left(\bar{u}_{\nu} \gamma_{\mu} u_{e}\right)$ where $G$ is the coupling constant, $u_{x}$ the Dirac spinor of the particle $x$, and the $\gamma_{\mu}$ are the Dirac gamma matrices. This contains no propagator, implying the four particles interact at a point. Fermi also implicitly assumed parity conservation, hence the vector-vector form of the interaction.

With the discovery of Parity violation, the vector-only nature of the weak interaction was questioned and a series of papers in $1957[33,34]$ led to the inclusion of an equal axial component $\left(\gamma_{\mu} \gamma_{5}\right)$ in the weak current, resulting in a $\beta$-decay matrix element of $\frac{G}{\sqrt{2}}\left[\bar{u}_{n} \gamma^{\mu}\left(1-\gamma_{5}\right) u_{p}\right]\left[\bar{u}_{\nu} \gamma_{\mu}\left(1-\gamma_{5}\right) u_{e}\right]$.

Without a propagator, Fermi theory predicts the cross section $\sigma(\nu n) \propto E_{\nu}^{2}$ (where $E_{\nu}$ is the neutrino energy). This violates unitarity at around $300 \mathrm{GeV}[35]$ (first noticed by Heisenberg [36] in 1936). However Hideki Yukawa had already introduced the concept of exchange bosons in the context of the strong force [37] which, when applied to the weak interaction, solved the unitarity problem (a further addition, such as that of the Higgs boson, being required to solve the problem across all energies). The boson needed to be massive so that the interaction was short-range. The mathematical problems of introducing this boson were solved in the 1960s by Sheldon Glashow [38], Abdus Salam [39] and Steven Weinberg [40]. At the same time they unified the weak and electromagnetic forces, predicted a 

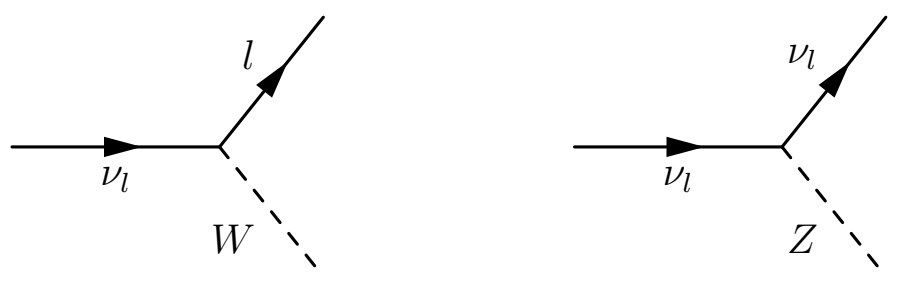

Figure 2.1: The possible interactions of a neutrino. Left: the charged current weak interaction. Right: the neutral current weak interaction. These interactions apply for all (non-sterile) neutrinos and antineutrinos of lepton flavour $l$.

neutral component to the weak interaction and predicted masses for the exchange bosons.

The neutral current (NC) was first observed in a neutrino interaction in the Gargamelle bubble chamber in 1973 [41, 42], the bubble chamber having been placed in the path of neutrino and antineutrino beams at CERN. The charged and neutral exchange bosons (the $W$ and $Z$ ) were first observed and their masses measured at the UA1 and UA2 experiments on the $p \bar{p}$ collider at CERN [43, 44, $45,46]$.

The neutrino, being electrically neutral and non-hadronic, is left to interact only through the weak interaction by the charged and neutral currents as in figure 2.1.

\subsection{Three Types of Neutrino}

The muon was discovered by Carl Anderson and Seth Neddermeyer in 1936 [47, 48]. It was, at first, thought to be the meson (now known as the pion) postulated by Yukawa to mediate the nuclear binding force. However, experiment [49] showed that the muon did not experience the strong interaction [50, 51]. This suggested the muon was a heavier version of the electron, particularly as it was found to undergo the same processes as the electron with similar coupling constants [52, 53]. The electron, muon and neutrino formed a family of particles known as leptons.

The lack of the decay $\mu \rightarrow e \gamma$ [54] suggested a separate conservation law for the 
number of electron and muon-type leptons, raising the question of whether there were separate electron and muon-type neutrinos. This question was answered at Brookhaven in 1962 [55] with the first neutrino beam. Colliding a proton beam on a beryllium target, a pion-rich (unfocused) hadronic beam was produced. The pions decayed through $\pi^{ \pm} \rightarrow \mu^{ \pm}+(\nu / \bar{\nu})$, the muons being stopped by shielding. The neutrinos, when interacting in spark chambers, were seen to produce only muons and no electrons: confirming their muon-like identity (the $\nu_{\mu}$ ).

The discovery of this heavier version of the electron led physicists to wonder if further, even heavier leptons existed [56]. The $\tau$ lepton was discovered in the SPEAR $e^{+} e^{-}$collider in 1975 [57]. The natural next question to ask was whether this new lepton also came with an associated neutrino.

The technical difficulties of producing tau neutrinos proved so large that the first evidence for it was an indirect measurement from the Large Electron Positron (LEP) collider at CERN. Comparing the total width of the $Z$ resonance to the width from its decays to visible particles yields the 'invisible width': the width for its decay to particles not visible in the detector, namely neutrinos. Assuming equal coupling strengths of the $Z$ to all neutrino types, fits to all LEP data yield a value of the number of neutrinos types lighter than $\frac{M_{Z}}{2}$ which experience the weak interaction of $2.994 \pm 0.012$ [58].

The $\nu_{\tau}$ was not directly observed until 2001 [59]. The DONUT collaboration, at Fermilab, created a $\nu_{\tau}$-enriched neutrino beam by colliding a proton beam on a thick tungsten target. The thick target absorbed most pions and kaons, leaving a beam rich in $D_{s}$ mesons, which decay with a $6 \%$ branching fraction to $\tau \nu_{\tau}$. After shielding to remove all non-neutrino particles, the beam passed through a detector of alternating steel and emulsion planes. $\nu_{\tau}$ charged current (CC) interactions took the form $\nu_{\tau}+N \rightarrow \tau^{-}+X$ (where $N$ is a nucleus in the detector and $X$ a hadronic shower). The tau is tracked in the emulsion, but decays after only a few millimetres into charged particles and neutrinos, causing a kink in the track visible with the 
high-resolution emulsion. Four such events were observed.

\subsection{Neutrino Flavour Change and Mass}

By 1957 the mass of the neutrino had not been measured to differ from zero, and many thought it was identically zero [60]. However, Bruno Pontecorvo considered the potential for neutrino flavour change allowed by a non-zero mass [61]: at this point $\nu \rightleftharpoons \bar{\nu}$ oscillations by analogy with the kaon system, assuming no conservation of lepton number in the (then one-flavour) neutrino sector. In 1967 [62], following the discovery of the $\nu_{\mu}$, he considered a number of different neutrino flavour change models including possible $\nu_{e} \rightleftharpoons \nu_{\mu}$ oscillations. Meanwhile, in 1964, Ziro Maki, Masami Nakagawa and Shoichi Sakata discussed the two-flavour neutrino oscillation model in the form we know it today (albeit in an attempt to explain the structure of hadronic particles alternate to the quark model) [63].

\subsubsection{Neutrino Oscillations}

The formulism of neutrino oscillations put forward by Pontecorvo, Maki, Nakagawa and Sakata (PMNS) was for a two flavour scenario. Here, it is extended to encompass three flavours, using the notation of [58].

Neutrinos experience the weak interactions in eigenstates of lepton flavour: $\left|\nu_{e}\right\rangle,\left|\nu_{\mu}\right\rangle$ and $\left|\nu_{\tau}\right\rangle$. They propagate through vacuum in their mass eigenstates $\left|\nu_{1}\right\rangle$, $\left|\nu_{2}\right\rangle$ and $\left|\nu_{3}\right\rangle$. These states are not equivalent, but are related by a rotation

$$
\left(\begin{array}{c}
\nu_{e} \\
\nu_{\mu} \\
\nu_{\tau}
\end{array}\right)=U^{*}\left(\begin{array}{l}
\nu_{1} \\
\nu_{2} \\
\nu_{3}
\end{array}\right)
$$

$U$ is called the PMNS rotation matrix, satisfying $U^{\dagger} U=\mathbb{1}$. The unitarity con- 
straint removes nine free parameters, and five of the remaining are relative phases between the six lepton fields which can be absorbed by those fields. This leaves four free parameters in $U$. The standard parameterisation is in terms of three mixing angles $\theta_{12}, \theta_{23}$ and $\theta_{13}$ and a phase $\delta$ :

$$
\begin{aligned}
U= & \left(\begin{array}{ccc}
U_{e 1} & U_{e 2} & U_{e 3} \\
U_{\mu 1} & U_{\mu 2} & U_{\mu 3} \\
U_{\tau 1} & U_{\tau 2} & U_{\tau 3}
\end{array}\right) \\
= & \left(\begin{array}{ccc}
1 & 0 & 0 \\
0 & c_{23} & s_{23} \\
0 & -s_{23} & c_{23}
\end{array}\right)\left(\begin{array}{ccc}
c_{13} & 0 & s_{13} e^{-\mathrm{i} \delta} \\
0 & 1 & 0 \\
-s_{13} e^{\mathrm{i} \delta} & 0 & c_{13}
\end{array}\right)\left(\begin{array}{ccc}
c_{12} & s_{12} & 0 \\
-s_{12} & c_{12} & 0 \\
0 & 0 & 1
\end{array}\right) \\
= & \left.\begin{array}{ccc}
c_{12} c_{13} \\
-s_{12} c_{23}-c_{12} s_{23} s_{13} e^{\mathrm{i} \delta} & c_{12} c_{23}-s_{12} s_{23} s_{13} e^{\mathrm{i} \delta} & s_{23} c_{13} \\
s_{13} e^{-\mathrm{i} \delta} & \\
s_{12} s_{23}-c_{12} c_{23} s_{13} e^{\mathrm{i} \delta} & -c_{12} s_{23}-s_{12} c_{23} s_{13} e^{\mathrm{i} \delta} & c_{23} c_{13}
\end{array}\right)
\end{aligned}
$$

where $s_{i j}=\sin \theta_{i j}$ and $c_{i j}=\cos \theta_{i j}$.

Any source (the Sun, a reactor, an accelerator, etc.) produces a neutrino at a time $t=0$ in a weak eigenstate $\left|\nu_{\alpha}(t=0)\right\rangle$. This is a sum of mass eigenstates $\left|\nu_{i}\right\rangle$ :

$$
\left|\nu_{\alpha}(0)\right\rangle=\sum_{i} U_{\alpha i}^{*}\left|\nu_{i}\right\rangle
$$

(At this point the treatment is general for any number of neutrino flavours.)

As the neutrino propagates, its mass eigenstates evolve:

$$
\left|\nu_{\alpha}(t)\right\rangle=\sum_{i} U_{\alpha i}^{*} e^{\mathrm{i} p_{i} \cdot x}\left|\nu_{i}\right\rangle
$$

where $x$ is the four-position of the neutrino and $p_{i}$ the four-momentum of the mass state $i$.

At a time $t$ the neutrino is observed through its weak interaction in a detector. 
Its wavefunction is therefore collapsed into a weak eigenstate $\left\langle\nu_{\beta}\right|=\sum_{j} U_{\beta j}\left\langle\nu_{j}\right|$ (a sum over mass eigenstates $j$ ):

$$
\begin{aligned}
\left\langle\nu_{\beta} \mid \nu_{\alpha}(t)\right\rangle & =\sum_{j} \sum_{i} U_{\beta j} U_{\alpha i}^{*} e^{\mathrm{i} p_{i} \cdot x}\left\langle\nu_{j} \mid \nu_{i}\right\rangle \\
& =\sum_{i} U_{\beta i} U_{\alpha i}^{*} e^{\mathrm{i} p_{i} \cdot x} .
\end{aligned}
$$

Labelling the energy and mass of the $i$ th neutrino mass eigenstate $E_{i}$ and $m_{i}$ respectively and assuming all mass eigenstates have the same three-momentum ${ }^{2}$ p

$$
\begin{aligned}
p_{i} \cdot x & =E_{i} t-\mathbf{p} \cdot \mathbf{x} \\
& =t \sqrt{|\mathbf{p}|^{2}+m_{i}^{2}}-\mathbf{p} \cdot \mathbf{x}
\end{aligned}
$$

Performing a binomial expansion assuming $m_{i} \ll E_{i}$ where, for highly relativistic neutrinos, $t=L$ and $\mathbf{p} \cdot \mathbf{x}=|\mathbf{p}| L$ (where $L=$ distance travelled):

$$
\begin{aligned}
p_{i} \cdot x & =|\mathbf{p}| L\left(1+\frac{m_{i}^{2}}{2|\mathbf{p}|^{2}}\right)-|\mathbf{p}| L \\
& =\frac{m_{i} L}{2 E} \quad\left(\text { as }|\mathbf{p}| \approx E \text { for } m_{i} \ll E_{i} \text { where } E \text { is the average of all } E_{i}\right) .
\end{aligned}
$$

$\operatorname{Thus}\left\langle\nu_{\beta} \mid \nu_{\alpha}(L)\right\rangle=\sum_{i} U_{\beta i} U_{\alpha i}^{*} e^{i \frac{m_{i}^{2} L}{2 E}}$.

The probability of observing a neutrino of flavour $\nu_{\beta}$ a distance $L$ from a source

\footnotetext{
${ }^{2}$ This is an approximation, as one could equally assume the three eigenstates have the same energy. A more exact treatment is the wave-packet approach [64].
} 
of originally pure $\nu_{\alpha}$ is then

$$
\begin{aligned}
P\left(\nu_{\alpha} \rightarrow \nu_{\beta}\right)= & \left|\left\langle\nu_{\beta} \mid \nu_{\alpha}(L)\right\rangle\right|^{2} \\
= & \left(\sum_{j} U_{\beta j}^{*} U_{\alpha j} e^{-\mathrm{i} \frac{m_{j}^{2} L}{2 E}}\right)\left(\sum_{i} U_{\beta i} U_{\alpha i}^{*} e^{\mathrm{i} \frac{m_{i}^{2} L}{2 E}}\right) \\
= & \sum_{i} \sum_{j} U_{\beta j}^{*} U_{\beta i} U_{\alpha i}^{*} U_{\alpha j} e^{-\mathrm{i} \frac{\Delta m_{i j}^{2} L}{2 E}} \\
& {\left[+\sum_{i} \sum_{j} U_{\beta j}^{*} U_{\beta i} U_{\alpha i}^{*} U_{\alpha j}-\sum_{i} \sum_{j} U_{\beta j}^{*} U_{\beta i} U_{\alpha i}^{*} U_{\alpha j}\right] }
\end{aligned}
$$

where $\Delta m_{i j}^{2}=m_{i}^{2}-m_{j}^{2}$; and the last two terms, being identical, sum to zero, and have been added in to enable the following manipulation. Rearranging:

$$
P\left(\nu_{\alpha} \rightarrow \nu_{\beta}\right)=\sum_{i} \sum_{j} \underbrace{U_{\beta j}^{*} U_{\beta i} U_{\alpha i}^{*} U_{\alpha j}\left(e^{-i \frac{\Delta m_{i j}^{2} L}{2 E}}-1\right)}_{B_{i j}}+\underbrace{\sum_{i} \sum_{j} U_{\beta j}^{*} U_{\beta i} U_{\alpha i}^{*} U_{\alpha j}}_{A} .
$$

Looking at the individual terms,

$$
\begin{aligned}
A & =\sum_{i} U_{\beta i} U_{\alpha i}^{*} \sum_{j} U_{\beta j}^{*} U_{\alpha j} \\
& =\sum_{i} U_{\beta i} U_{i \alpha}^{\dagger} \sum_{j} U_{j \beta}^{\dagger} U_{\alpha j} \\
& =\delta_{\alpha \beta}
\end{aligned}
$$

due to the unitarity of $U$. Looking at $B_{i j}$, we see that $B_{i j}=B_{j i}^{*}$ and $B_{i i}=0$. Thus $B_{i j}+B_{j i}=2 \mathfrak{R e}\left[B_{i j}\right]$. Therefore the terms in the sum over $B_{i j}$ can be paired together and those with $i=j$ removed:

$$
P\left(\nu_{\alpha} \rightarrow \nu_{\beta}\right)=\delta_{\alpha \beta}+2 \sum_{i(>j)} \sum_{j} \mathfrak{R e}\left[U_{\beta j}^{*} U_{\beta i} U_{\alpha i}^{*} U_{\alpha j}\left(e^{-\mathrm{i} \frac{\Delta m_{i j}^{2} L}{2 E}}-1\right)\right]
$$

Using

$$
e^{-\mathrm{i} \frac{\Delta m_{i j}^{2} L}{2 E}}=\cos \left(\frac{\Delta m_{i j}^{2} L}{2 E}\right)+\mathrm{i} \sin \left(\frac{\Delta m_{i j}^{2} L}{2 E}\right)
$$


and

$$
\cos \left(\frac{\Delta m_{i j}^{2} L}{2 E}\right)-1=2 \sin ^{2}\left(\frac{\Delta m_{i j}^{2} L}{4 E}\right)
$$

gives

$$
\begin{aligned}
P\left(\nu_{\alpha} \rightarrow \nu_{\beta}\right)= & \delta_{\alpha \beta} \\
& +2 \sum_{i(>j)} \sum_{j} \mathfrak{I m}\left[U_{\beta j}^{*} U_{\beta i} U_{\alpha i}^{*} U_{\alpha j}\right] \sin \left(\frac{\Delta m_{i j}^{2} L}{2 E}\right) \\
& -4 \sum_{i(>j)} \sum_{j} \mathfrak{R e}\left[U_{\beta j}^{*} U_{\beta i} U_{\alpha i}^{*} U_{\alpha j}\right] \sin ^{2}\left(\frac{\Delta m_{i j}^{2} L}{4 E}\right) .
\end{aligned}
$$

\subsubsection{The Two-Flavour Approximation}

In most experiments it is sufficient to use a two neutrino-flavour model. This can be derived by beginning with a $2 \times 2$ PMNS matrix and following the steps laid out above (see, for example, [65]). However, it is more informative to show how the three-flavour formulation reduces to a two-flavour model as this demonstrates the necessary approximations and their validity.

As a demonstration, the case of the MINOS experiment (on which the analysis presented in this thesis is based) will be used. This produces a beam of muon neutrinos of energy $\mathcal{O}(3 \mathrm{GeV})$ and measures the number of muon neutrinos remaining after they have propagated $735 \mathrm{~km}$.

So far, units have been used in which $c=\hbar=1$. Moving into SI units, the argument of the last sinusoidal term in equation 2.3 becomes

$$
\frac{\Delta m_{i j}^{2} c^{4} L}{4 E \hbar c}
$$

To leave $E$ in units of $\mathrm{GeV}, L$ in $\mathrm{km}$ and $\Delta m_{i j}^{2}$ in $\mathrm{eV}^{2}$, putting $\hbar=6.58 \times$ $10^{-25} \mathrm{GeVs}, c=3.00 \times 10^{5} \mathrm{~km} \mathrm{~s}^{-1}$ and $\Delta m_{i j}^{2} c^{4}=\Delta m_{i j}^{2} \times 10^{-18}\left(\mathrm{eV}^{2} / \mathrm{GeV}^{2}\right)$, the 
argument then becomes

$$
\frac{1.27 \Delta m_{i j}^{2} L}{E}
$$

and the argument of the first sinusoidal term in equation 2.3 is a factor of two larger.

The transition probability relevant to MINOS is

$$
P\left(\nu_{\mu} \rightarrow \nu_{\mu}\right)=1-4 \sum_{i(>j)} \sum_{j}\left|U_{\mu i}\right|^{2}\left|U_{\mu j}\right|^{2} \sin ^{2}\left(\frac{1.27 \Delta m_{i j}^{2} L}{E}\right)
$$

where the $\mathfrak{I m}$ term in equation 2.3 goes to zero when $\alpha=\beta$. As shall be seen later in this chapter, experiment has found $\sin \theta_{13}$ to be small. Approximating $\sin \theta_{13}=$ 0 and $\cos \theta_{13}=1$, the relevant PMNS matrix components from equation 2.2 are

$$
\begin{array}{ll}
U_{\mu 1}=s_{12} c_{23}-c_{12} s_{23} s_{13} e^{\mathrm{i} \delta} & \Rightarrow\left|U_{\mu 1}\right|^{2} \approx s_{12}^{2} c_{23}^{2} \\
U_{\mu 2}=c_{12} c_{23}-s_{12} s_{23} s_{13} e^{\mathrm{i} \delta} & \Rightarrow\left|U_{\mu} 2\right|^{2} \approx c_{12}^{2} c_{23}^{2} \\
U_{\mu 3}=s_{23} c_{13} & \Rightarrow\left|U_{\mu 3}\right|^{2} \approx s_{23}^{2} .
\end{array}
$$

Experiment has shown $\Delta m_{21}=\mathcal{O}\left(7 \times 10^{-5} \mathrm{eV}^{2}\right)$. Thus for MINOS,

$$
\sin ^{2}\left(\frac{1.27 \Delta m_{21}^{2} L}{E}\right) \approx \sin ^{2}\left(\frac{1.27 \times 7 \times 10^{-5} \times 735}{3}\right) \approx \sin ^{2}(0.02) \approx 0
$$

Similarly, $\Delta m_{31}^{2}=\mathcal{O}\left(3 \times 10^{-3} \mathrm{eV}^{2}\left(\gg \Delta m_{21}^{2}\right)\right)$ and $\Delta m_{31}^{2}-\Delta m_{21}^{2}=\Delta m_{32}^{2}$, so $\Delta m_{31}^{2} \approx \Delta m_{32}^{2}=\Delta m_{\mathrm{atm}}^{2}$. Therefore,

$$
P\left(\nu_{\mu} \rightarrow \nu_{\mu}\right) \approx 1-4 s_{23}^{2} c_{23}^{2}\left(s_{12}^{2}+c_{12}^{2}\right) \sin ^{2}\left(\frac{1.27 \Delta m_{\mathrm{atm}}^{2} L}{E}\right)
$$

Using $2 \sin \theta_{23} \cos \theta_{23}=\sin 2 \theta_{23}$ and $\sin ^{2} \theta_{12}+\cos ^{2} \theta_{12}=1$ gives the well known 
two-flavour expression for the neutrino survival probability:

$$
P\left(\nu_{\mu} \rightarrow \nu_{\mu}\right)=1-\sin ^{2}\left(2 \theta_{23}\right) \sin ^{2}\left(\frac{1.27 \Delta m_{\mathrm{atm}}^{2} L}{E}\right)
$$

\subsection{Evidence for Neutrino Oscillations}

\subsubsection{Solar Neutrinos}

The first fuel for the idea of neutrino oscillations came in 1968 in the form of an experiment performed by Ray Davis at the Homestake mine. Davis measured the flux of neutrinos from ${ }^{8} \mathrm{~B}$ decay in the Sun by looking for atoms of Argon produced in a tank of $\mathrm{C}_{2} \mathrm{Cl}_{4}$ through the process $\nu_{e}+{ }^{37} \mathrm{Cl} \rightarrow e^{-}+{ }^{37} \mathrm{Ar}$. He put a limit of less than half the flux expected from solar models [66] (as he detected no neutrinos). The solar models [67], particularly relating to the Boron chain, were not well trusted at the time; so a mistake in the solar model was viewed as the most likely cause (although Pontecorvo and Gribov did relate this result to their kaon-like model of neutrino oscillations [68]).

For the next 20 years little happened, despite Davis continuing taking data at Homestake, in which he detected neutrinos and confirmed his earlier result. Eventually, the Kamiokande water Cerenkov detector, built to search for proton decay, was upgraded in 1986 to detect ${ }^{8} \mathrm{~B}$ solar neutrinos through electron scattering ( $\nu_{x} e^{-} \rightarrow \nu_{x} e^{-}$; a process, as explained below, dominated by electron neutrinos). In 1989 the collaboration published a measurement of $0.46 \pm 0.13$ (stat.) \pm 0.08 (syst) times the flux expected from the standard solar model (SSM) [69].

Physicists decided to investigate the discrepancy by looking for lower energy neutrinos from the $p p$ solar chain. There is a large (up to 30\%) uncertainty on the ${ }^{8} \mathrm{~B}$ neutrinos flux, but the $p p$ flux is known to within $3 \%$ [70]. Hence fewer uncertainties will affect neutrino disappearance measurements made with these $p p$ 
neutrinos. These neutrinos could be observed with Gallium $\left(\nu_{e}+{ }^{71} \mathrm{Ga} \rightarrow e^{-}+{ }^{71} \mathrm{Ge}\right.$, with a neutrino energy threshold of $0.233 \mathrm{MeV}$ ) which was done by SAGE in 1991 [71] and GALLEX in 1992 [72]. The final results [73, 74] measured around half the flux of neutrinos predicted by the SSM. GALLEX continued on as GNO through to 2003 [75], measuring a neutrino flux slightly lower than, but consistent with, GALLEX.

The Kamiokande detector continued data taking through to 1995 [76], confirming a measurement of only half the expected ${ }^{8} \mathrm{~B}$ solar neutrino flux, with the additional features that the direction of the detected neutrinos could be correlated with the position of the Sun, and their energy spectrum shown to agree with the SSM prediction for the neutrino energy spectrum from ${ }^{8} \mathrm{~B}$ decay [77].

Kamiokande was succeeded by a larger version, Super-Kamiokande, which has 50, 000 tonnes of water [78] compared to Kamiokande's 3, 000 tonnes [76]. SuperKamiokande is still taking data, but their most recent solar neutrino results [79] again yield around half the expected ${ }^{8} \mathrm{~B}$ neutrino flux.

The SNO detector, which took data from 2000 to 2006, consists of a sphere of 1,000 tonnes of heavy water $\left(\mathrm{D}_{2} \mathrm{O}\right)$ surrounded by PMTs. The deuterium in the heavy water allows three different interaction mechanisms of the neutrinos to be observed. The CC process $\nu_{e}+d \rightarrow p+p+e^{-}$, the NC process $\nu_{x}+d \rightarrow n+p+\nu_{x}$, and the electron scattering process $\nu_{x}+e^{-} \rightarrow \nu_{x}+e^{-}$which happens through both the NC interactions of all neutrino flavours and the CC interactions of electron neutrinos $\left(\nu_{e}\right.$ interactions dominate this channel by a factor of $\sim 6$ ).

The electrons produced by the CC interactions are detected by observing their Cerenkov light in the water. The direction of the electrons from the electron scattering process are more strongly correlated with the direction of the Sun, so can be separated from those from the CC nuclear interaction on a statistical basis.

In the second phase of operations, two tonnes of $\mathrm{NaCl}$ were added to the heavy water. Neutrons produced in the NC process are captured by the ${ }^{35} \mathrm{Cl}$ to produce 


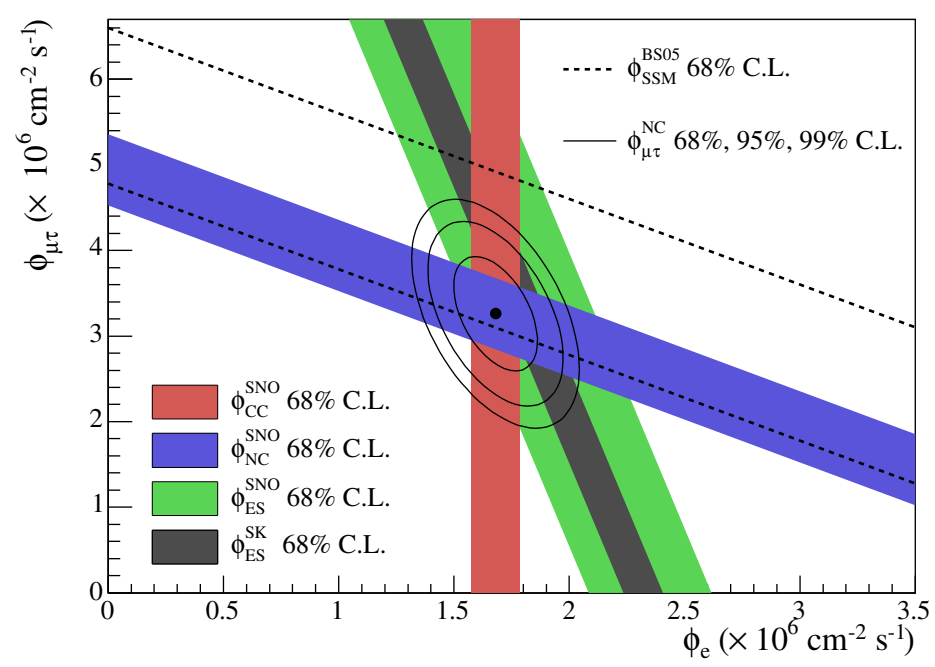

Figure 2.2: The flux $(\phi)$ of $\nu_{e}$ and $\nu_{\mu}+\nu_{\tau}$ from the Sun measured with the SNO detector. Dotted lines: region in which the $\nu_{\tau}+\nu_{\mu}+\nu_{e}$ fluxes sum to the SSM prediction. Blue: region in which the $\nu_{\tau}+\nu_{\mu}+\nu_{e}$ fluxes sum to the total flux measured from the NC interactions. Red: measured $\nu_{e}$ flux through the CC interaction with deuterium nuclei. Green: electron scattering measurement (NC and $\mathrm{CC}$, but with $\mathrm{CC}$ dominating); grey: the same electron scattering measurement from the Super-Kamiokande experiment. Figure from [80].

an excited state of ${ }^{36} \mathrm{Cl}$ which relaxes to its ground state emitting photons detected by the PMTs.

In the final phase, the salt was removed and ${ }^{3} \mathrm{He}$ proportional counters lowered into the detector, which have a high cross-section for neutron capture: again aiding the NC measurement.

The measurement of the NC interaction rate allowed the total flux of all active neutrino flavours to be measured: this should be equal to the $\nu_{e}$ flux predicted by the SSM if the deficit of electron neutrinos measured by the preceding experiments is due to neutrino flavour change. This is indeed what SNO measured as shown in figure 2.2. The figure shows the $\nu_{\mu}+\nu_{\tau}$ flux against the $\nu_{e}$ flux. The dotted lines show the region constrained such that the $\nu_{\tau}+\nu_{\mu}+\nu_{e}$ fluxes sum to the SSM prediction. The blue stripe shows the region constrained such that the $\nu_{\tau}+$ $\nu_{\mu}+\nu_{e}$ fluxes sum to the total measured by $\mathrm{SNO}$ from the $\mathrm{NC}$ interactions. The 
red stripe shows the $\nu_{e}$ flux measured by the CC nuclear process. The green stripe shows the electron scattering measurement, with the corresponding SuperKamiokande measurement superimposed in grey. The constraints all coincide in a region consistent with the SSM plus neutrino flavour change. The ratio of $\nu_{e}$ flux (from the nuclear CC process) to total neutrino neutrino flux was measured to be $0.340 \pm 0.023$ (stat.) ${ }_{-0.031}^{+0.029}$ (syst.) $[80]$.

\section{Interpretation of the Solar Data}

As demonstrated for MINOS in section 2.7.2, solar neutrino oscillations can also be treated in a two-flavour framework through the application of a similar set of approximations. The mass splitting and mixing angle relevant to solar oscillations are $\Delta m_{21}^{2}$ and $\theta_{12}$. The values of the parameters allowed by the data are summarised in figure 2.3.

The allowed regions from chlorine and gallium data include an area with $\Delta m_{21}^{2}<10^{-9} \mathrm{eV}^{2}$ and a large mixing angle $\left(0.1<\tan ^{2} \theta<10\right)$. This comes through an interpretation of simple oscillations in vacuum between the Sun and Earth, the wavelength of oscillations, $l_{v}$, having a value such that the Earth lies at a point at which the neutrino wavefunction has become predominantly non- $\nu_{e}$. The different energies of neutrinos probed by gallium and chlorine mean only a small region of parameter space satisfies both sets of data. The extreme case of this scenario at the lowest allowed $\Delta m_{21}^{2}$ corresponds to the Earth falling within one oscillation wavelength of the Sun, and is referred to as the just so solution as it requires a precise coincidence between $l_{v}$ and the Earth to Sun distance. The reasonably large mixing angle is required to account for the large $\nu_{e}$ deficit. $\theta_{12}$ enters equation 2.4 in the form $\sin ^{2}\left(2 \theta_{12}\right)$, hence $P\left(\nu_{e} \rightarrow \nu_{e}\right)$ is identical under $\theta_{12} \rightarrow\left(90^{\circ}-\theta_{12}\right)$. Thus the regions $\theta<45^{\circ}$ and $\theta>45^{\circ}$ cannot be resolved, hence the line of symmetry about $\tan ^{2} \theta=1$.

A further constraint on this vacuum solution comes from the non-observation 


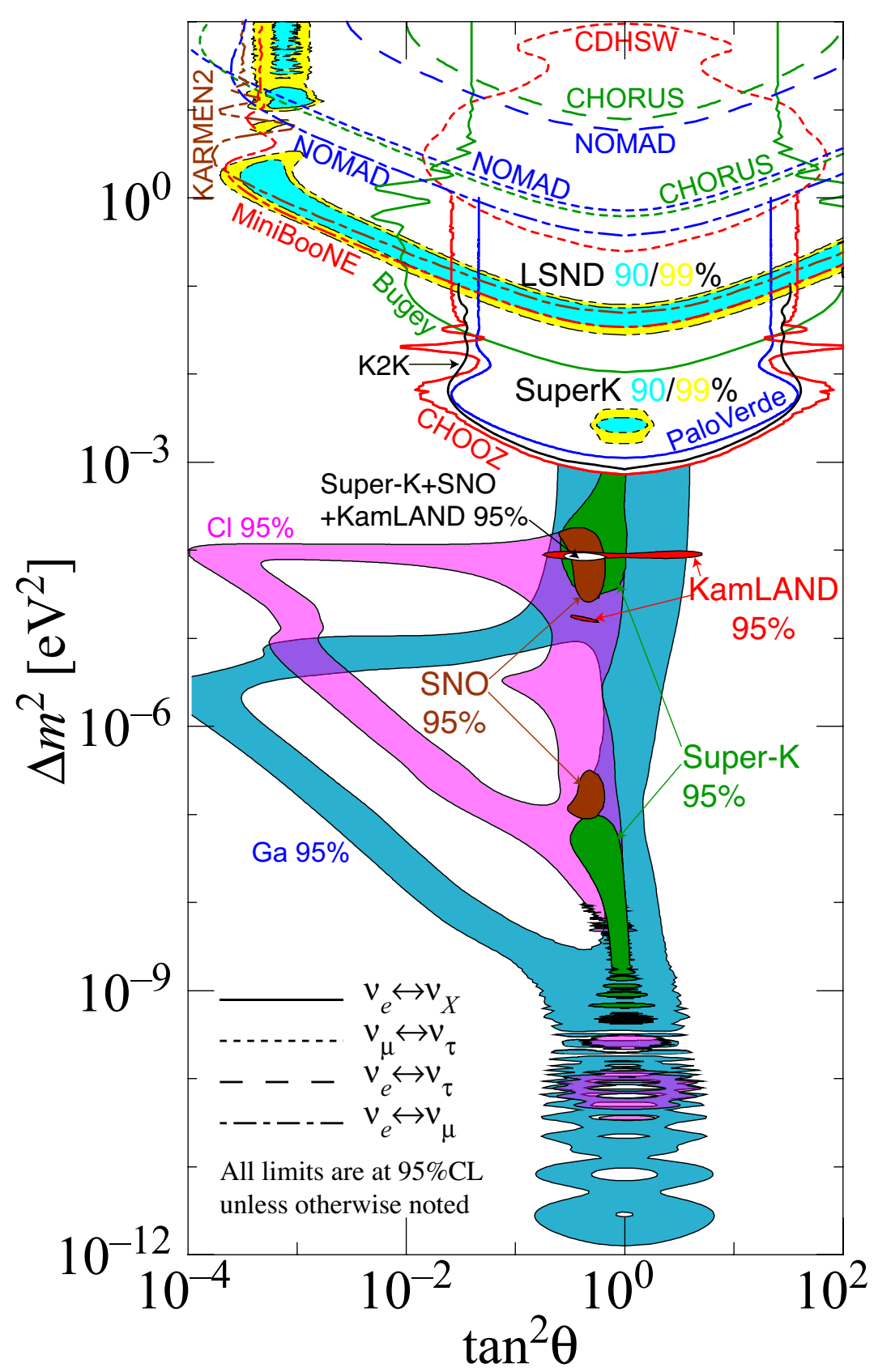

http://hitoshi.berkeley.edu/neutrino

Figure 2.3: A summary of the constraints on neutrino oscillation parameters afforded by all data to date. Filled, coloured areas represent allowed regions. Lines not surrounding filled regions are exclusion limits: when these lines do not close the region above the line is excluded, otherwise the region within the line is excluded. 
of seasonal variations in the $\nu_{e}$ flux by the water experiments: the eccentricity of the Earth's orbit alters the Sun to Earth distance, which would alter the relative amount of $\nu_{e}$ to non- $\nu_{e}$ component in the neutrino wavefunction.

From equation $2.4 l_{v}$ can be seen (in natural units) to be $\frac{4 \pi E}{\Delta m_{12}^{2}}$. As $\Delta m_{21}^{2}$ increases, $l_{v}$ decreases until the separate oscillation peaks cannot be resolved. What is observed is an average over the second ( $L$-dependent) sinusoidal term in equation 2.4 , giving

$$
\left.P\left(\nu_{e} \rightarrow \nu_{e}\right)\right|_{\text {small wavelength }}=1-\frac{1}{2} \sin ^{2}\left(2 \theta_{12}\right)>\frac{1}{2}
$$

hence the vertical stripe continuing into the higher $\Delta m_{21}^{2}$ region for the Gallium experiments (where the flux deficit was approximately $\frac{1}{2}$ ) but not for Chlorine or water where the $>\frac{1}{2}$ deficit cannot be explained in this region.

However, a further interpretation of the data exists beyond the simple vacuum oscillation model set out above.

Lincoln Wolfenstein first pointed out in 1978 [81] that the passage of neutrinos through dense matter affects the process of oscillation. Stanislav Mikeev and Alexei Smirnov [82] pointed out the application of this idea to solar neutrino oscillations.

In vacuum, neutrinos propagate as mass eigenstates. Thus the free-particle mass eigenstates are eigenstates of the free Hamiltonian

$$
\mathrm{i} \frac{\partial}{\partial t}\left(\begin{array}{c}
\nu_{1} \\
\nu_{2}
\end{array}\right)=\mathcal{H}_{0}\left(\begin{array}{c}
\nu_{1} \\
\nu_{2}
\end{array}\right)
$$

To transform this into an equation for flavour eigenstates the two-dimensional version of equation 2.1 is used (in two dimensions all phases in $U$ can be absorbed into the wavefunctions, leaving the matrix real). Also, assuming small neutrino 
mass,

$$
\mathcal{H}_{0} \nu_{i}=E \nu_{i}=\left(\sqrt{p^{2}+m_{i}^{2}}\right) \nu_{i} \approx\left(p+\frac{m_{i}^{2}}{2 p}\right) \nu_{1} \approx\left(p+\frac{m_{i}^{2}}{2 E}\right) \nu_{1}
$$

Substituting these into equation 2.6,

$$
\mathrm{i} \frac{\partial}{\partial t} U^{\dagger}\left(\begin{array}{c}
\nu_{e} \\
\nu_{x}
\end{array}\right)=\left[p \mathbb{1}+\left(\begin{array}{cc}
\frac{m_{1}^{2}}{2 E} & 0 \\
0 & \frac{m_{2}^{2}}{2 E}
\end{array}\right)\right] U^{\dagger}\left(\begin{array}{c}
\nu_{e} \\
\nu_{x}
\end{array}\right)
$$

where $E$ is the average energy of the two mass states and $\nu_{x}$ represents the non- $\nu_{e}$ neutrinos in the two-flavour approximation. Multiplying from the left by $U$ and $\begin{aligned} \text { using } U= & \left(\begin{array}{cc}\cos \theta & \sin \theta \\ -\sin \theta & \cos \theta\end{array}\right) \\ & \mathrm{i} \frac{\partial}{\partial t}\left(\begin{array}{c}\nu_{e} \\ \nu_{x}\end{array}\right)=\left[\left(p+\frac{m_{1}^{2}}{2 E}\right) \mathbb{1}+U\left(\begin{array}{cc}0 & 0 \\ 0 & \frac{\Delta m_{21}^{2}}{2 E}\end{array}\right) U^{\dagger}\right]\left(\begin{array}{c}\nu_{e} \\ \nu_{x}\end{array}\right) \\ = & {\left[\left(p+\frac{m_{1}^{2}}{2 E}+\frac{1}{2}\right) \mathbb{1}-\left(\begin{array}{cc}\cos (2 \theta) & -\sin (2 \theta) \\ -\sin (2 \theta) & -\cos (2 \theta)\end{array}\right) \frac{\Delta m_{21}^{2}}{4 E}\right]\left(\begin{array}{c}\nu_{e} \\ \nu_{x}\end{array}\right) . }\end{aligned}$

The interaction of the neutrinos in matter is modelled by adding a potential term to the Hamiltonian. All active flavours experience the same potential $\left(V_{N}\right)$ through their NC interactions, but the electron neutrinos experience an extra potential, $-G N_{e} \sqrt{2}$, through their CC interactions with electrons in the matter ( $G$ is the Fermi constant, $N_{e}$ the number density of electrons in the matter). The Hamiltonian in the flavour basis must be correspondingly transformed:

$$
\mathcal{H}_{0}\left(\begin{array}{c}
\nu_{e} \\
\nu_{x}
\end{array}\right) \rightarrow\left[\mathcal{H}_{0}+\left(\begin{array}{cc}
V_{N}-G N_{e} \sqrt{2} & 0 \\
0 & V_{N}
\end{array}\right)\right]\left(\begin{array}{c}
\nu_{e} \\
\nu_{x}
\end{array}\right)
$$


Performing this transformation on equation 2.8 (taking all diagonal terms into a constant $K)$,

$$
\begin{aligned}
\mathrm{i} \frac{\partial}{\partial t}\left(\begin{array}{c}
\nu_{e} \\
\nu_{x}
\end{array}\right)= & \\
& {\left[K \mathbb{1}-\frac{\Delta m_{21}^{2}}{4 E}\left(\begin{array}{cc}
\cos 2 \theta+\frac{G N_{e}}{\sqrt{2}} \frac{4 E}{\Delta m_{21}^{2}} & -\sin 2 \theta \\
-\sin 2 \theta & -\cos 2 \theta-\frac{G N_{e}}{\sqrt{2}} \frac{4 E}{\Delta m_{21}^{2}}
\end{array}\right)\right]\left(\begin{array}{c}
\nu_{e} \\
\nu_{x}
\end{array}\right) . }
\end{aligned}
$$

To interpret these results analogously to vacuum oscillations, this equation should be put into the form of equation 2.7. This can be done to obtain

$$
U \rightarrow\left(\begin{array}{cc}
\cos \theta_{m} & \sin \theta_{m} \\
-\sin \theta_{m} & \cos \theta_{m}
\end{array}\right) ; \quad \frac{\Delta m_{21}^{2}}{2 E} \rightarrow \frac{\Delta m_{m}^{2}}{2 E}
$$

with

$$
\tan 2 \theta_{m}=\frac{\sin 2 \theta}{\cos 2 \theta-\frac{l_{v}}{l_{0}}}
$$

where $l_{0}=\frac{2 \pi}{G N_{e} \sqrt{2}}$.

Neutrinos are produced near the very dense (high $N_{e}$ ) centre of the Sun and move outwards through gradually decreasing $N_{e}$, which gradually changes $\theta_{m}$. The neutrinos are therefore only ever in instantaneous mass eigenstates $\nu_{m 1, m 2}$, related to their flavour states by

$$
\left(\begin{array}{c}
\nu_{m 1} \\
\nu_{m 2}
\end{array}\right)=\left(\begin{array}{cc}
\cos \theta_{m} & -\sin \theta_{m} \\
\sin \theta_{m} & \cos \theta_{m}
\end{array}\right)\left(\begin{array}{c}
\nu_{e} \\
\nu_{x}
\end{array}\right)
$$

If the change in density is slow enough to be considered adiabatic, the neutrinos remain in these instantaneous eigenstates as they propagate. (This adiabatisity condition requires the oscillation wavelength in matter, $l_{m}=\frac{4 \pi E}{\Delta m_{m}^{2}}$, to be much smaller than the length of characteristic density changes.) 
Taking the case in which $\Delta m_{21}^{2}>0$ and $\theta<45^{\circ}$, for a high enough $N_{e}$, equation 2.9 implies $\tan 2 \theta_{m} \rightarrow 0$ from below, hence $\theta_{m} \rightarrow 90^{\circ}$, independent of $\theta$. Thus $\nu_{e}=\nu_{m 2}$, so neutrinos produced at the centre of the Sun are produced mainly in the $\nu_{m 2}$ state. As the neutrinos propagate outwards, $N_{e}$ decreases adiabatically and the neutrinos stay in the $\nu_{m 2}$ state as $\theta_{m}$ decreases back to $\theta$ when they reach the vacuum of space, at which point $\nu_{m 2}=\nu_{2}$. Taking a situation of very small $\theta$, $\nu_{2} \approx \nu_{x}$. Thus upon reaching Earth a large $\nu_{e}$ deficit $(>50 \%)$ is observed.

In figure 2.3 a triangular allowed region is visible in the Chlorine and Gallium data. Above the horizontal line at the top of the triangles, $\Delta m_{21}^{2}$ is so large that $N_{e}$ at the core of the Sun is not high enough to allow $\theta_{m}$ to be different enough from $\theta$ to account for the $\nu_{e}$ deficit. To the right of the triangles, $\theta>45^{\circ}$ and for large $N_{e}, \tan \theta_{m} \rightarrow 0$ from above (so $\theta_{m} \rightarrow 0$ ) independent of the sign of $\Delta m_{21}^{2}$, suppressing rather than enhancing the oscillations. In the centre of the triangles the MSW effect is too strong, causing a deficit in $\nu_{e}$ larger than measured in data. The left most point of the triangles mark the point at which $\theta$ is so small that $N_{e}$ in the core of the Sun is too small to enhance $\theta_{m}$ enough to cause the $\nu_{e}$ deficit. Below the diagonal side of the triangles the adiabatic approximation breaks down. The density changes fast enough that as $\theta_{m}$ returns to $\theta$, the neutrinos do not stay entirely in $\nu_{m 2}$. At the surface, some are left in the $\nu_{2}$ state and some in the $\nu_{1}$ state. At the low values of $\sin \theta$ this means there is a higher $\nu_{e}$ component to the wavefunction than for pure $\nu_{m 2}$, hence a smaller deficit.

For $\Delta m_{21}^{2}<0$ a high $N_{e}$ causes $\theta_{m} \rightarrow 0$, hence a suppression rather than the observed enhancement of the oscillations. Thus the solar data resolves the hierarchy of these two neutrino mass states.

The Gallium and Chlorine triangles fall in different regions due to the different energies of the neutrinos involved. The combined data therefore rules out much of the parameter space, leaving regions at a small mixing angle (SMA; $\tan ^{2} \theta_{12} \sim$ $10^{-3}$ ), the vacuum region (VAC; $\Delta m_{21}^{2}<10^{-9}$ ), a low $\Delta m_{21}^{2}$ region $\left(\mathrm{LOW} ; \Delta m_{21}^{2} \sim\right.$ 
$10^{-7}$ ) and a large mixing angle region (LMA; $\Delta m_{21}^{2} \sim 10^{-4}$ ).

The water Cerenkov detectors give two further pieces of information due to their abilities to measure the energy spectrum and time of day of the neutrinos. The shape of the neutrinos energy spectrum is expected to differ from that predicted by the SSM as, at a low enough energy, the matter effect is no longer strong and vacuum oscillations apply. Only a very small spectral distortion was measured. Furthermore, passage through the Earth causes the matter effect to come into play, again enhancing the mixing angle, allowing a regeneration of electron neutrinos at night when the Earth is between the detector and the Sun. SNO measured no significant day-night asymmetry in the CC $\nu_{e}$ interaction rate [80]. These results exclude the SMA region and cut into the LOW, VAC and LMA regions, leaving the LMA region most favoured.

\section{Manmade 'Solar' Neutrinos}

The remaining ambiguities in parameter space were removed by the KamLAND experiment. This observes the flux of $\bar{\nu}_{e}$ from the various nuclear reactors around Japan, using 1 kTonne of liquid scintillator. The energy and baseline $\frac{L}{E}$ is such that the experiment is sensitive to the LMA region. The most recent result [83] is shown in figure 2.4. The result clearly shows an oscillatory pattern of $\bar{\nu}_{e}$ disappearance as a function of $\frac{L}{E}$, covering two oscillation maxima. This is a conclusive signature of the LMA solution and furthermore disfavours alternative models of neutrino flavour change (such as decoherence and decay).

The current world best fit to all solar-regime oscillation data gives $\Delta m_{21}^{2}=$ $\left(8.0_{-0.4}^{+0.6}\right) \times 10^{-5} \mathrm{eV}^{2}$ and $\theta_{12}=\left(33.9_{-2.2}^{+2.4}\right)^{\circ}[58]$.

\section{The Future: Low Energy Solar Neutrinos}

The next step in solar neutrino physics is to look for the matter to vacuum oscillation transition to confirm the LMA solution from solar neutrinos and test the 


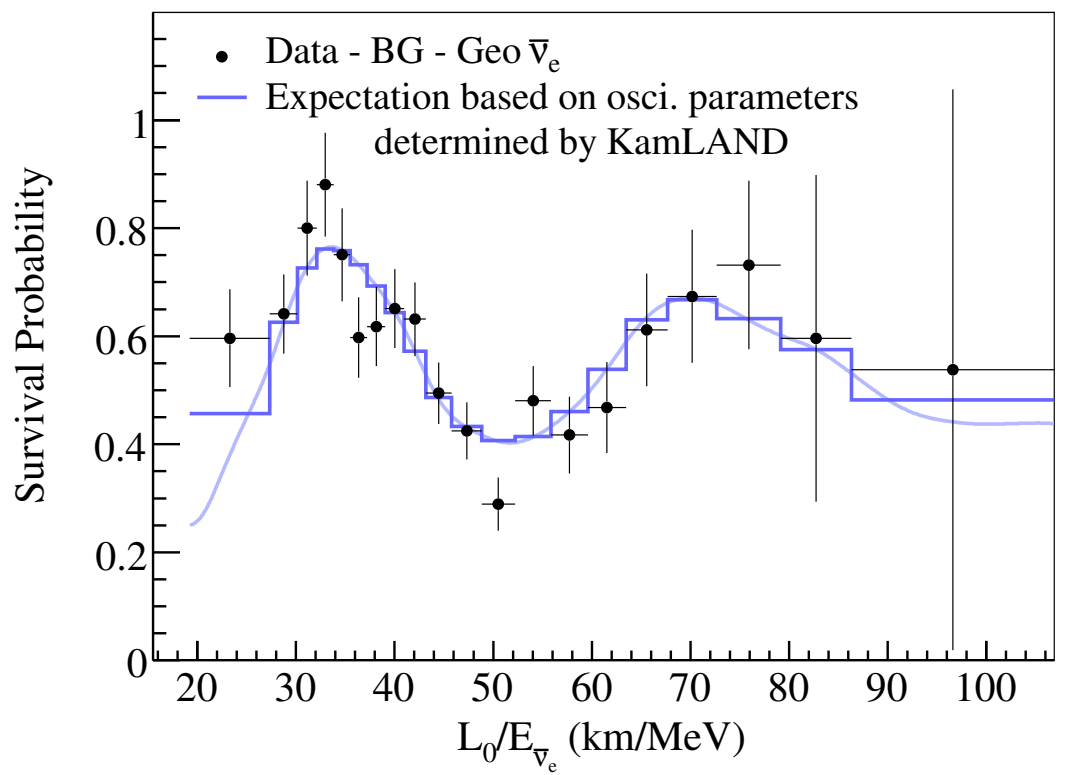

Figure 2.4: The most recent results of the KamLAND experiment. The ratio of measured to expected $\bar{\nu}_{e}$ flux is show (black dots) as a function of distance travelled divided by neutrino energy. The best fit of the oscillation hypothesis is shown in blue. Figure taken from [83].

theory of the matter effect. For the LMA solution this transition is expected to happen in the region of $1 \mathrm{MeV}$. Looking at such low-energy solar neutrinos requires very low background, liquid scintillator detectors. Such a detector is Borexino [84] which has recently published its first results consistent with the LMA solution [85]. A similar experiment is SNO+ [86], expected to start taking data in 2010. Larger liquid scintillator detectors are planned for the more distant future, for example LENA [87].

\subsubsection{Atmospheric Neutrinos}

Cosmic rays consist of protons and other nuclei which interact with the Earth's atmosphere to produce a flux of neutrinos. The primary process involved in neutrino production is $\pi \rightarrow \mu \nu_{\mu}$ followed by $\mu \rightarrow e \nu_{\mu} \nu_{e}$ (with both charges of pion contributing approximately equally; kaon decay contributes at a lower level but through an identical channel). This means that, whilst the absolute flux of neutrinos is known only to within $20 \%$ below $10 \mathrm{GeV}$, the ratio $R=\left(\nu_{\mu}+\bar{\nu}_{\mu}\right.$ flux $) /\left(\nu_{e}+\bar{\nu}_{e}\right.$ flux $) \approx 2$ 
is known with much higher accuracy (better than 2\%) in this energy range [88].

The experiments that first investigated atmospheric neutrinos in the context of oscillations had been built to look for proton decay. Atmospheric neutrinos were an important background to these experiments, encouraging their study.

In 1988 Kamiokande (the water Cerenkov experiment introduced above in the context of solar neutrinos) produced independent measurements of the atmospheric $\nu_{e}$ and $\nu_{\mu}$ fluxes, observing the expected $\nu_{e}$ flux but a $(56 \pm 7) \%$ deficit of $\nu_{\mu}$ interactions [89]. However, this was not considered conclusive proof of atmospheric $\nu_{\mu}$ disappearance as two iron calorimeter experiments published results consistent with no disappearance: NUSEX measured $R_{\text {data }} / R_{\text {expected }}=0.96_{-0.28}^{+0.32}[90]$ in 1989 , and Fréjus measured $R_{\text {data }} / R_{\text {expected }}=1.00 \pm 0.15$ (stat.) \pm 0.08 (syst.) [91] in 1995 .

In 1992 another water Cerenkov experiment, the IMB detector, released results in which they measured no $\nu_{\mu}$ disappearance [92], giving confidence to the iron calorimeter collaborations. However, in 1992 IMB produced a further set of results yielding $R=1.4$ with a $2.6 \sigma$ deviation from $R=2$ [93]. Kamiokande had also continued to take data during this time, and by 1992 had measured a double ratio $R_{\text {data }} / R_{\text {expected }}=0.60_{-0.06}^{+0.07}$ (stat.) \pm 0.05 (syst.) $[94]$.

The possibility that this was a systematic error associated with water Cerenkov detectors was ruled out when two further iron calorimeters measured a neutrino flux differing from the expectation: MACRO in 1995 [95] and Soudan2 in 1997 [96].

To more directly test the oscillation hypothesis requires observation of the neutrino flux as a function of $\frac{L}{E}$. This is possible by looking at the flux as a function of zenith angle: neutrinos produced directly above the detector typically travel $L \sim$ $15 \mathrm{~km}$ whereas those produced on the far side of the earth travel $L \sim 10^{4} \mathrm{~km}$ before detection. This was first attempted by Kamiokande in 1994 [97], showing some zenith-angle dependence of $R$; and, more recently, by Super-Kamiokande: first in 1998 [98] giving convincing evidence for neutrino mass, with ongoing analysis [99].

Super-Kamiokande was also introduced earlier in the context of solar neutri- 
nos. Like other water Cerenkov detectors it has the ability to differentiate $\nu_{e}$ and $\nu_{\mu} \mathrm{CC}$ interactions from the charged lepton produced. Electrons shower as they pass through the water, producing a diffuse Cerenkov ring. Muons pass almost unhindered (barring ionisation losses), producing a much sharper ring. Zenith angle measurements from Super-Kamiokande are shown in figure 2.5 [100]. No deficit in any neutrino flux is seen in the down-going neutrinos, suggesting they have not travelled enough distance for oscillations to occur. The upwards-going muon neutrinos show a depletion of $\sim 50 \%$, suggesting they have travelled the distance of many periods of oscillation and the condition of equation 2.5 has been reached: averaging the sinusoidal term over $\frac{L}{E}$, with $\sin ^{2} 2 \theta_{23} \sim 1$. (The approximations of section 2.7.2 apply in obtaining the two-flavour oscillation model, implying the relevant mixing angle is $\theta_{23}$ and the mass difference $\Delta m_{\mathrm{atm}}^{2}$.) No deviation from the expectation is seen in the electron neutrinos, showing that the $\nu_{\mu}$ disappearance is not occurring through $\nu_{\mu} \rightarrow \nu_{e}$ transitions.

Super-Kamiokande produced a further analysis [101], binning the data as a

function of $\frac{L}{E}$ resolution, obtaining the results shown in figure 2.6. An $\frac{L}{E}$-dependent deficit in $\nu_{\mu}$ flux is visible, with neutrino oscillations providing a better parameterisation than any alternative model (neutrino decay [102] is disfavoured at $3.4 \sigma$, decoherence [103] at 3.8\%).

\section{Manmade Neutrino Beams}

As with solar neutrinos, using manmade neutrinos gives a more controlled situation in which to test the oscillation hypothesis. The $\mathrm{K} 2 \mathrm{~K}$ experiment created a beam of muon neutrinos from a proton beam at $12 \mathrm{GeV}$ and sent them $250 \mathrm{~km}$ to the Super-Kamiokande detector. The neutrino energy spectrum was measured near the production point (before oscillations) by a smaller water Cerenkov detector. Comparing this with the energy spectrum measured at Super-Kamiokande revealed an energy dependent $\nu_{\mu}$ disappearance characteristic of oscillations [104]. 

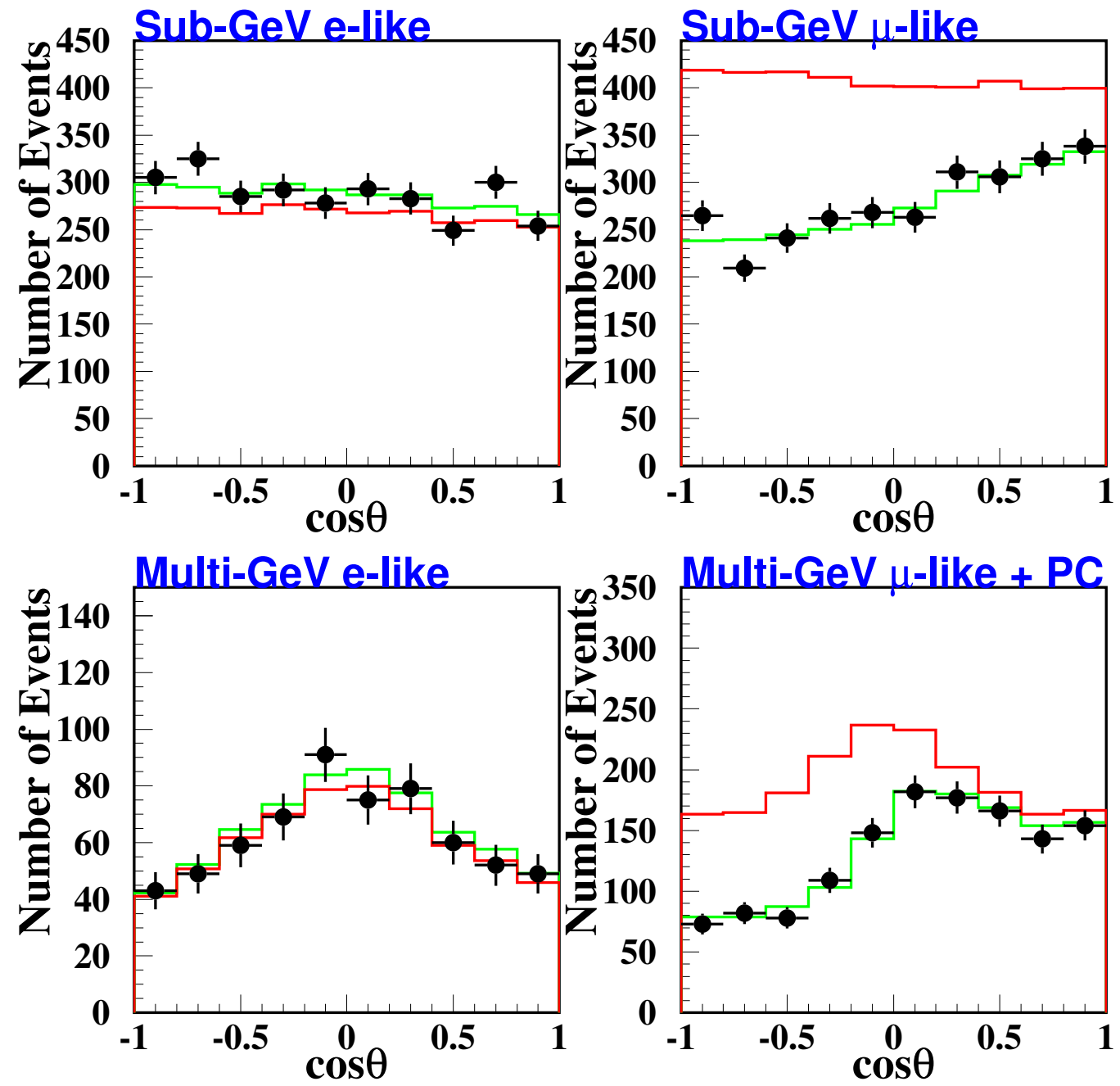

Figure 2.5: The atmospheric neutrino event rate measured by the SuperKamiokande detector as a function of zenith angle. Left column: $\nu_{e}$-like events; right column: $\nu_{\mu}$-like events. Top row: high energy events; bottom row: low energy events. Black dots: data; red line: predicted event rate without oscillation; green line: predicted event rate with best fit to oscillations. Figure taken from [100]. 


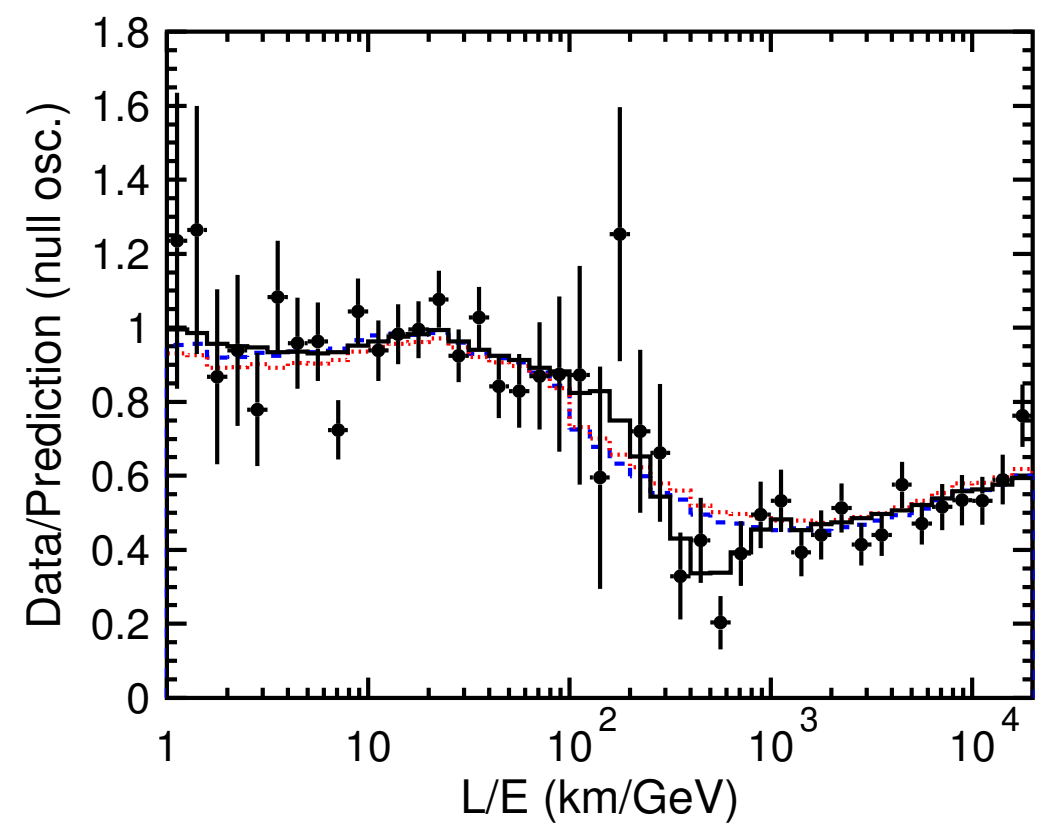

Figure 2.6: Super-Kamiokande data. Black points: the ratio of the measured atmospheric neutrino event rate to that expected in the no oscillation case as a function of the distance travelled divided by energy of the neutrino $(L / E)$. Black line: the predicted ratio with the best fit to oscillations. Red and blue lines: the predicted ratio with the best fit to decoherence and neutrino decay respectively. Figure taken from [101]. 
The MINOS experiment (discussed at length through the rest of this thesis) follows a similar method, using iron calorimeters. The proton beam is at $120 \mathrm{GeV}$ and the baseline a correspondingly longer $735 \mathrm{~km}$.

\section{Interpretation of the Data}

The Super-Kamiokande zenith angle data (figure 2.5) show a deficit of muon neutrinos but no excess of electron neutrinos, showing that the transition occurring is not $\nu_{\mu} \rightarrow \nu_{e}$. In the assumption of no extra, sterile types of neutrinos, this implies the process is $\nu_{\mu} \rightarrow \nu_{\tau}$.

All atmospheric neutrino (and $\nu_{\mu}$ beam) data can be modelled in the twoflavour scheme set out in section 2.7.2. The interpretation is simpler than in the solar case as matter effects do not play a role: only the $\nu_{\mu}$ and $\nu_{\tau}$ flavours are involved which experience identical (NC only) matter potentials.

The regions in parameter space allowed by the most precise experiments are shown in figure 2.7, including those from the MINOS experiment [105]. (The Super-Kamiokande contours are also included in figure 2.3.)

The current official world average of the atmospheric oscillation parameters (made in 2006 before the release of MINOS results) gives, at 90\% c.l., $1.9 \times$ $10^{-3} \mathrm{eV}^{2}<\Delta m_{\mathrm{atm}}^{2}<3.0 \times 10^{-3} \mathrm{eV}^{2}$ and $\sin ^{2} 2 \theta_{23}>0.90$. Figure 2.7 shows the MINOS results to be consistent with these numbers.

\section{The Future: Accuracy, $\nu_{\tau}$ Appearance and the Mass Hierarchy}

MINOS will continue to take data until at least 2010, improving the accuracy of its $\Delta m_{\mathrm{atm}}^{2}$ measurement. The projected (statistical only) sensitivities for various exposures are shown in figure 2.8. A current estimate would be a final exposure in the region of $8-10 \times 10^{20}$ protons on target $(\mathrm{PoT})$.

These efforts will be continued by two new long-baseline manmade $\nu_{\mu}$ beam experiments. Nova [106] will use the same beam as MINOS, but sit about $2^{\circ}$ 


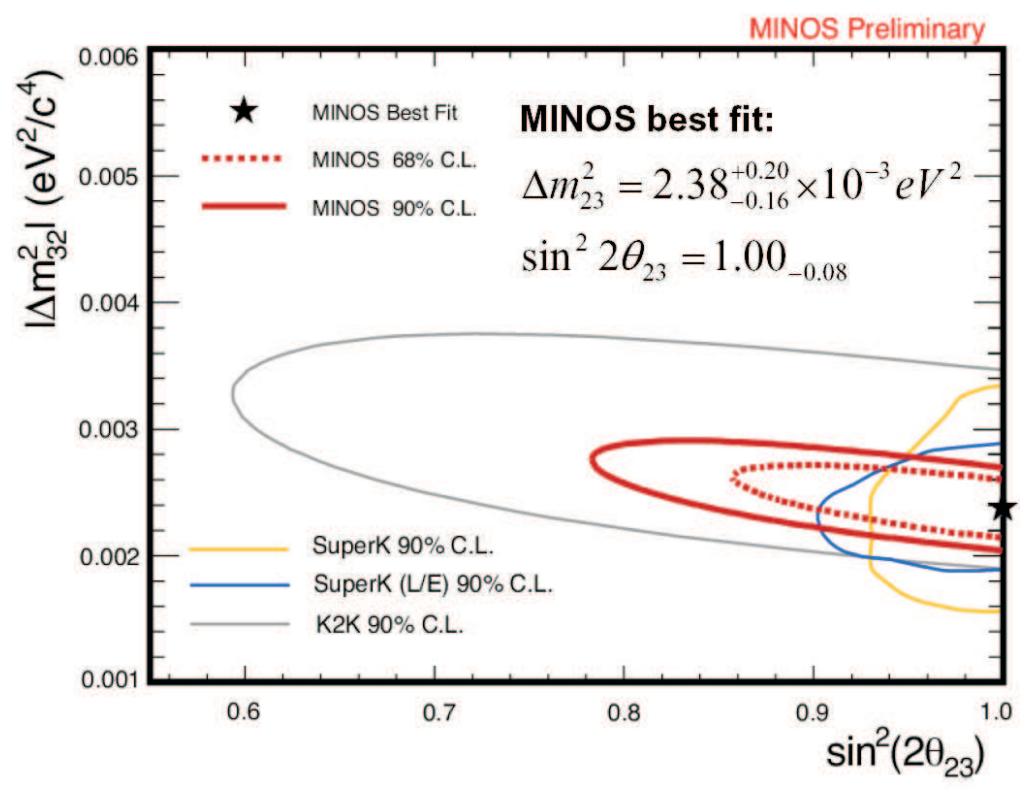

Figure 2.7: The regions in atmospheric neutrino oscillation parameter space allowed by the most accurate experiments. The grey contour is the region allowed by K2K [104], the yellow and blue from two Super-Kamiokande analyses [99, 101]. The red contours and best fit point are from the MINOS experiment [105].

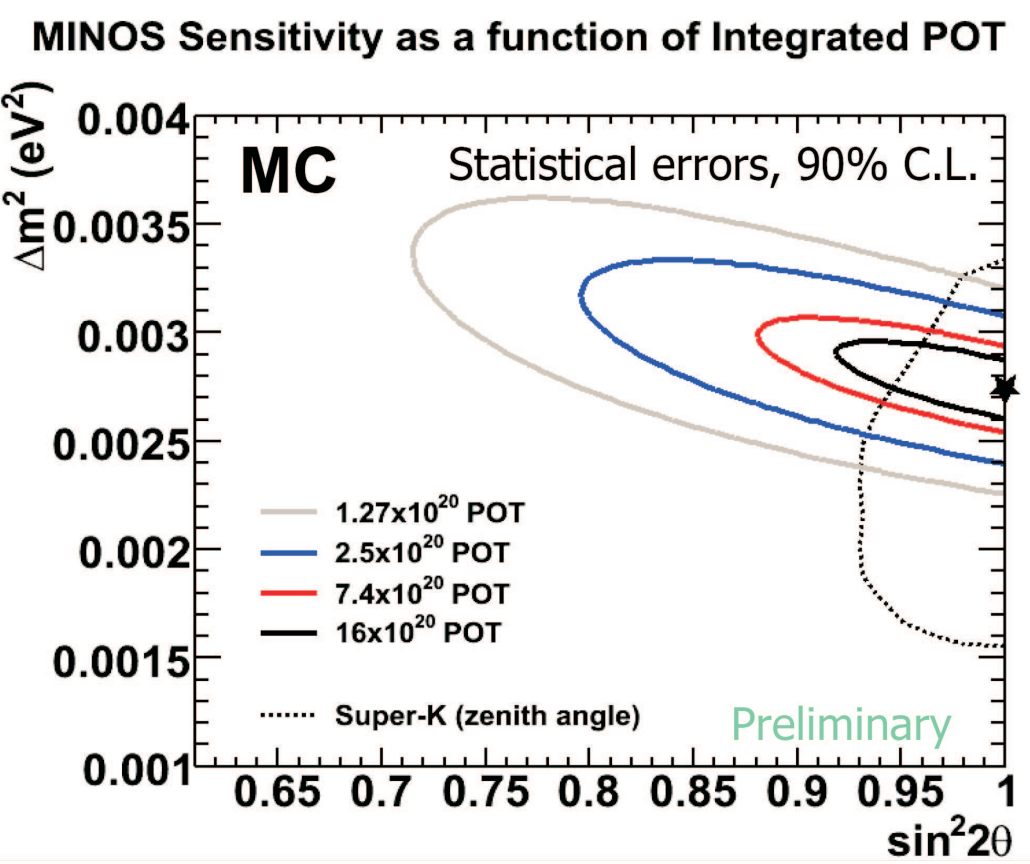

Figure 2.8: Projected, statistical only sensitivities for various MINOS exposures (in units of protons on target: POT). For reference is shown the allowed region from the current Super-K zenith angle analysis [99]. 
off the beam axis to reduce background from neutrinos in the high-energy tail of the beam. Liquid scintillator near and far detectors will be used, the far detector being anywhere between 13 kTonnes and 30 kTonnes.

A similar off-axis concept will be T2K [107] in Japan, which follows the K2K model: using a $50 \mathrm{GeV}$ proton beam to send muon neutrinos $295 \mathrm{~km}$ to the

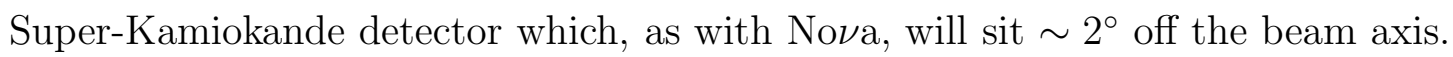
However, the T2K near detector will not be a water Cerenkov instrument. It will consist of an upstream fine-grained lead calorimeter, optimised to investigate $\pi^{0}$ production; and a downstream part to look at the tracks of charged particles, consisting of TPCs interleaved with water and plastic scintillator.

Precisions of the order of $10^{-4} \mathrm{eV}^{2}$ on $\Delta m_{\mathrm{atm}}^{2}$ and 0.01 on $\sin ^{2} 2 \theta_{23}$ are expected from this generation of experiments.

It has been assumed that the process $\nu_{\mu} \rightarrow \nu_{\tau}$ is responsible for the observed $\nu_{\mu}$ disappearance (and the Super-Kamiokande collaboration have released analyses showing the consistency of their data with $\nu_{\tau}$ appearance [108]), but the appearance of tau neutrinos has yet to be directly observed. The OPERA detector [109] (which has recently started taking data [110]) aims to do this, receiving the CERN to Gran Sasso (CNGS) $\nu_{\mu}$ beam after $732 \mathrm{~km}$. Using a technique similar to that of DONUT, finely grained ( $\sim 1 \mathrm{~mm}$ ) lead sheets are interleaved with $50 \mu \mathrm{m}$ emulsion layers on a $200 \mu \mathrm{m}$ plastic base. This high position resolution will be able to identify the short $(\mathcal{O}(1 \mathrm{~mm}))$ tracks left by decaying tau leptons indicative of $\nu_{\tau}$ $\mathrm{CC}$ interactions.

As electron neutrinos are not involved in atmospheric oscillations, the matter effect does not come into play. The data can therefore be interpreted with the vacuum oscillation model which is independent of the sign of $\Delta m_{\mathrm{atm}}^{2}$. However, a small component of $\nu_{\mu} \rightarrow \nu_{e}$ transitions may occur through the angle $\theta_{13}$ (which will be discussed in more detail in section 2.8.3). This $\nu_{e}$ component will introduce matter effects through the model discussed in the solar case (section 2.8.1), 
dependent on the sign of $\Delta m_{\mathrm{atm}}^{2}$. The potential introduced to the Hamiltonian is different for neutrinos and antineutrinos, so observing separately $\nu_{\mu} \rightarrow \nu_{e}$ and $\bar{\nu}_{\mu} \rightarrow \bar{\nu}_{e}$ transitions may allow the mass hierarchy to be resolved: an observation that the Nova experiment may address. (However, for this measurement to be possible will require a non-zero value of $\theta_{13}$.)

\subsection{3 $\theta_{13}$}

The remaining unmeasured oscillation parameter is the mixing angle $\theta_{13}$. This drives $\nu_{e} \leftrightarrow \nu_{\mu}$ oscillations through the mass splitting $\Delta m_{\text {atm }}^{2}$ (therefore at a smaller $\frac{L}{E}$ than the solar transitions, allowing the two regimes to decouple).

The $\frac{L}{E}$ scale is well suited to the observation of reactor neutrinos at a distance of $\mathcal{O}(1 \mathrm{~km})$. The best limit of this type has been produced by the Chooz experiment: a liquid scintillator detector (a technique similar to that used by KamLAND) placed $1 \mathrm{~km}$ from the Chooz B nuclear power station in northern France. The final results in 2003 [111] observed no signal, giving the exclusion region shown in figure 2.3 .

The lack of signal means $\theta_{13}$ is small. The current world best average of $\theta_{13}$ measurements gives a $90 \%$ c.l. limit of $\sin ^{2}\left(2 \theta_{13}\right)<0.19$ [58]. (A global analysis including constraints from solar and atmospheric experiments takes this limit down to $\sin ^{2}\left(2 \theta_{13}\right)<0.07$ [112], the constraints from the solar and atmospheric data arising from removal of the two-flavour oscillation approximation used in the interpretation discussed in previous sections.)

$\theta_{13}$ can also be measured by looking at the process $\nu_{\mu} \rightarrow \nu_{e}$ through the atmospheric mass splitting. The MINOS experiment will look for this $\nu_{e}$ appearance in the near future. Its sensitivity to $\theta_{13}$ is shown in figure 2.9 (as a function of $\delta$ which will be discussed in section 2.12).

The Nova and T2K experiments discussed above will additionally search for 


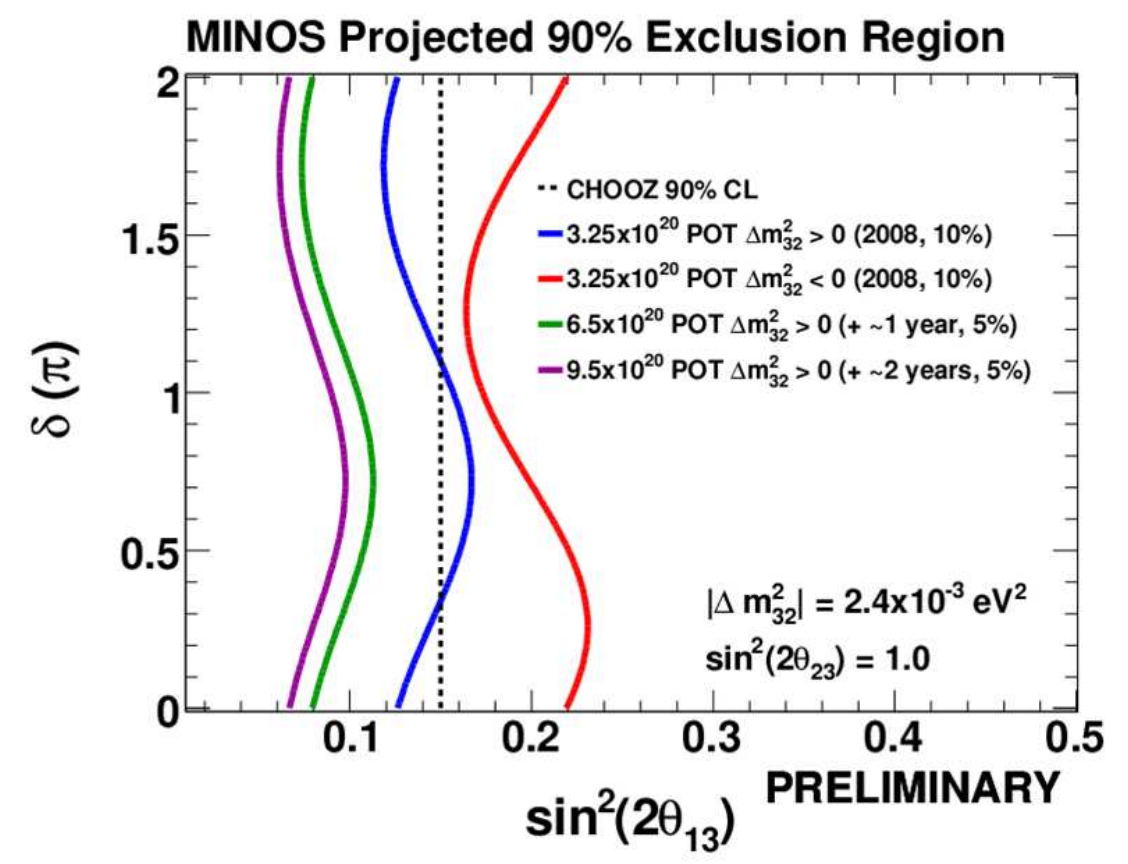

Figure 2.9: The predicted sensitivity of MINOS to the mixing angle $\theta_{13}$, depending on the sign of $\Delta m_{\mathrm{atm}}^{2}$ (see legend). The sensitivity is also a function of the $\mathcal{C P}$ violating phase $\delta$ (see section 2.12). The current limit from the Chooz experiment [111] is shown (black dotted line) for reference.

$\theta_{13}$ through the $\nu_{e}$ appearance channel. Reactor experiments are also planned to look through the $\nu_{e}$ disappearance channel following the Chooz model. These include Double Chooz [113] and Daya Bay [114], which have the ability to place $90 \%$ c.l. upper limits on $\sin ^{2}\left(2 \theta_{13}\right)$ of 0.025 and 0.01 respectively (or to make a positive measurement).

\section{$2.9 \mathcal{C P}$ Violation}

\subsection{1 $\mathcal{C}$ Violation}

The operation of charge conjugation (given the symbol $\mathcal{C}$ ) involves swapping all particles for antiparticles. The discovery of the preferred helicity of the neutrino by Goldhaber, as well as being an expression of parity $(\mathcal{P})$ violation, also demonstrates $\mathcal{C}$ violation. All neutrinos are found to be left handed whilst antineutrinos 
are right handed. Under charge conjugation, this situation reads as all neutrinos being right handed and antineutrinos left handed: precisely what is not observed in nature.

\subsection{2 $\mathcal{C P}$ Conjugation}

Interpretation of the Goldhaber results suggests nature may still be symmetric following the application of $\mathcal{C}$ followed by $\mathcal{P}$, or $\mathcal{C P}$. A left handed neutrino under $\mathcal{C}$ becomes a left handed antineutrino. Applying $\mathcal{P}$ transforms this to a right handed antineutrino: the observed manifestation.

\subsubsection{The Discovery of $\mathcal{C P}$ Violation}

The neutral kaon system gave an opportunity for the conservation of $\mathcal{C P}$ to be tested. The neutral kaons are not themselves $\mathcal{C P}$ eigenstates:

$$
\mathcal{C P}\left|K^{0}\right\rangle=\left|\bar{K}^{0}\right\rangle \quad \mathcal{C P}\left|\bar{K}^{0}\right\rangle=\left|K^{0}\right\rangle
$$

However, $\mathcal{C P}$ eigenstates can be constructed:

$$
\begin{array}{ll}
\left|K_{1}\right\rangle=\frac{1}{\sqrt{2}}\left(\left|K^{0}\right\rangle+\left|\bar{K}^{0}\right\rangle\right) & \mathcal{C P}\left|K_{1}\right\rangle=\left|K_{1}\right\rangle \\
\left|K_{2}\right\rangle=\frac{1}{\sqrt{2}}\left(\left|K^{0}\right\rangle-\left|\bar{K}^{0}\right\rangle\right) & \mathcal{C P}\left|K_{2}\right\rangle=-\left|K_{2}\right\rangle .
\end{array}
$$

Two kaon decay modes were observed, into two $\mathcal{C P}$ eigenstates:

$$
\begin{array}{ll}
K_{1} \rightarrow 2 \pi, & \text { lifetime }=(0.8953 \pm 0.0005) \times 10^{-8} \mathrm{~s}, \\
K_{2} \rightarrow 3 \pi, & \text { lifetime }=(5.114 \pm 0.021) \times 10^{-8} \mathrm{~s},
\end{array}
$$

the $3 \pi$ mode having the longer lifetime due to the smaller phase space. 
In 1964, James Cronin and Val Fitch allowed a beam of kaons to propagate for a distance long enough to ensure they were all of the $K_{2}$ variety. Measuring the energies of two of the pions from the decays of the remaining kaons should give the continuous energy distribution characteristic of a three-body decay. However, a peak of excess events at the maximum possible energy (corresponding to the kaon mass) was observed, signifying a small number of two-body decays. This showed that some of the $\mathcal{C P}=-1$ particles had decayed through the $\mathcal{C P}=+1$ channel: direct evidence for $\mathcal{C P}$ violation [115].

\section{$2.10 \mathcal{C P} \mathcal{T}$ Invariance}

A further observation can be introduced: that of time reversal, $\mathcal{T}$. It is a fundamental result from quantum field theory that nature must be invariant under the combined operation $\mathcal{C P} \mathcal{T}$ (provided nature obeys locality and unitarity, and is Lorentz invariant) [116].

$\mathcal{C P} \mathcal{T}$ invariance implies the masses and lifetimes of particles and antiparticles must be equal. The best test comes from the mass difference between the $K^{0}$ and $\bar{K}^{0}$. The current limit is [58]

$$
\frac{\left|m_{K^{0}}-m_{\bar{K}^{0}}\right|}{m_{\text {average }}}<10^{-18} \quad(90 \% \text { c.l. }) \text {. }
$$

\section{$2.11 \mathcal{T}$ Invariance}

As $\mathcal{C P}$ is violated, a corresponding violation of $\mathcal{T}$ is required to maintain $\mathcal{C P} \mathcal{T}$ conservation. The CPLEAR experiment observed a difference between the oscillation rates $K^{0} \rightarrow \bar{K}^{0}$ and $\bar{K}^{0} \rightarrow K^{0}$ which they claimed was a direct observation of $\mathcal{T}$ violation [117]. It has been questioned whether this is indeed a direct observation [118], but is still viewed as such by many (for example [119] in which 
the level of $\mathcal{T}$ observed is judged of the correct level to be consistent with $\mathcal{C P} \mathcal{T}$ invariance).

An observation of the electric dipole moment (EDM) of the neutron would be evidence of $\mathcal{T}$ violation. The only preferred direction for a neutron is the direction of its spin. The EDM must therefore be aligned (or anti-aligned) with this direction. Upon time reversal, the spin changes direction but an EDM would not, creating a different state of the neutron. The standard model predicts (from $\mathcal{C P}$ violating weak interaction effects) an $\mathrm{EDM}$ of $\mathcal{O}\left(10^{-31} e \mathrm{~cm}\right)\left(e=-1.6 \times 10^{-19} \mathrm{C}\right)$. Theories of physics beyond the standard model can bring this prediction up to $\mathcal{O}\left(10^{-25} e \mathrm{~cm}\right)[120]$. The current world limit on the measured neutron EDM is $<2.9 \times 10^{-26} e \mathrm{~cm}$ at $90 \%$ c.l. $[121]$.

\section{$2.12 \mathcal{C P}$ Violation in the Neutrino Sector}

If $\mathcal{C P}$ is violated then, assuming $\mathcal{C P} \mathcal{T}$ conservation, $\mathcal{T}$ is violated. Applying the $\mathcal{T}$ operator to neutrino oscillations,

$$
\mathcal{T}\left(\nu_{\alpha} \rightarrow \nu_{\beta}\right)=\left(\nu_{\beta} \rightarrow \nu_{\alpha}\right)
$$

To calculate the effect of this transformation on the oscillation probability, $\alpha$ and $\beta$ should be exchanged in equation 2.3. This has the effect of changing the sign of the $\mathfrak{I m}$ term in that equation. Taking the complex conjugate of the PMNS matrix $U$ has exactly the same effect on equation 2.3 and the only effect this has on $U$ is to change the sign of the phase $\delta$ : hence $\delta$ is known as the $\mathcal{C P}$ violating phase.

Detecting $\mathcal{C P}$ violation in neutrino oscillations involves making a measurement of $\delta$. However, equation 2.2 shows that $\delta$ only ever appears multiplied by $\sin \theta_{13}$. Section 2.8.3 discussed the experimental data showing that $\theta_{13}$ is very small. $\delta$ can only be observed if a non-zero $\theta_{13}$ is observed. 
Looking at equation 2.3 in the case of a disappearance measurement (setting $\beta=\alpha$ ) shows that only the modulus squared of PMNS matrix elements appear in the disappearance probability. Thus $\delta$ does not appear, so $\mathcal{C P}$ violation cannot be observed in disappearance experiments such as Double Chooz and Daya Bay. This is not true for appearance experiments. The action of $\mathcal{C P}$ on appearance oscillations is (notating neutrino helicity with a subscript $L, R$ for (left,right)handedness)

$$
\begin{aligned}
\mathcal{C P}\left(\nu_{\alpha, L} \rightarrow \nu_{\beta, L}\right) & =\mathcal{C}\left(\nu_{\alpha, R} \rightarrow \nu_{\beta, R}\right) \\
& =\left(\bar{\nu}_{\alpha, R} \rightarrow \bar{\nu}_{\beta, R}\right)
\end{aligned}
$$

expressed in the oscillation model by the transformation $U \rightarrow U^{*}$ (or $\delta \rightarrow-\delta$ ). Thus $\mathcal{C P}$ violation can be observed by looking for differences between the $\nu_{\mu} \rightarrow$ $\nu_{e}$ and $\bar{\nu}_{\mu} \rightarrow \bar{\nu}_{e}$ oscillation probabilities. The No $\nu$ a experiment may make this measurement.

This coupling of $\theta_{13}$ with $\delta$ in the $\nu_{e}$ appearance case explains why, in section 2.8.3, limits on $\theta_{13}$ from such experiments were always given as a function of $\delta$.

\section{$2.13 \mathcal{C P} \mathcal{T}$ Violation in the Neutrino Sector}

$\mathcal{C P} \mathcal{T}$ violation would manifest itself in neutrino oscillations as differing mixing angles or mass differences, $\theta_{i j}$ or $\Delta m_{i j}^{2}$, between neutrinos and antineutrinos. No such effect has yet been observed but the current limits, summarised in [122], are weak.

Limits on the solar mixing parameters come from combining the solar neutrino 
data with the KamLAND antineutrino data, giving (at $3 \sigma$ )

$$
\begin{gathered}
\left|\sin ^{2} \theta_{12}-\sin ^{2} \bar{\theta}_{12}\right|<0.3 \\
\left|\Delta m_{21}^{2}-\Delta \bar{m}_{21}^{2}\right|<1.1 \times 10^{-4} \mathrm{eV}^{2} .
\end{gathered}
$$

For the atmospheric mixing parameters, the limits come from a combination of the MINOS neutrino data, and the Super-Kamiokande data which is a mixture of neutrino and antineutrino data, but dominated by the neutrinos due to their higher cross section. The resulting limits are (at $3 \sigma)$

$$
\left|\sin ^{2} \theta_{23}-\sin ^{2} \bar{\theta}_{23}\right|<0.45,\left|\Delta m_{\mathrm{atm}}^{2}-\Delta \bar{m}_{\mathrm{atm}}^{2}\right|<1 \times 10^{-2} \mathrm{eV}^{2}
$$

The limit on $\bar{\theta}_{13}$ comes from the CHOOZ experiment, and that on $\theta_{13}$ from a combination of the solar and atmospheric regime neutrino data:

$$
\left|\sin ^{2} \theta_{13}-\sin ^{2} \bar{\theta}_{13}\right|<0.3
$$

again at $3 \sigma$.

The MINOS detector has already produced limits on $\mathcal{C P} \mathcal{T}$ violation using atmospheric neutrinos [123]. These limits on the atmospheric mass difference, which are very weak, are shown in figure 2.10 and assume maximal mixing (i.e. $\left.\sin ^{2}\left(2 \theta_{23}\right)=1\right)$. The current world limits on this mass difference, which will be further investigated in this thesis, are presented graphically in [124], the graph reproduced in figure 2.11. The allowed values for $\Delta \bar{m}_{\text {atm }}^{2}$ cover more than an order of magnitude at $90 \%$ c.l.. 


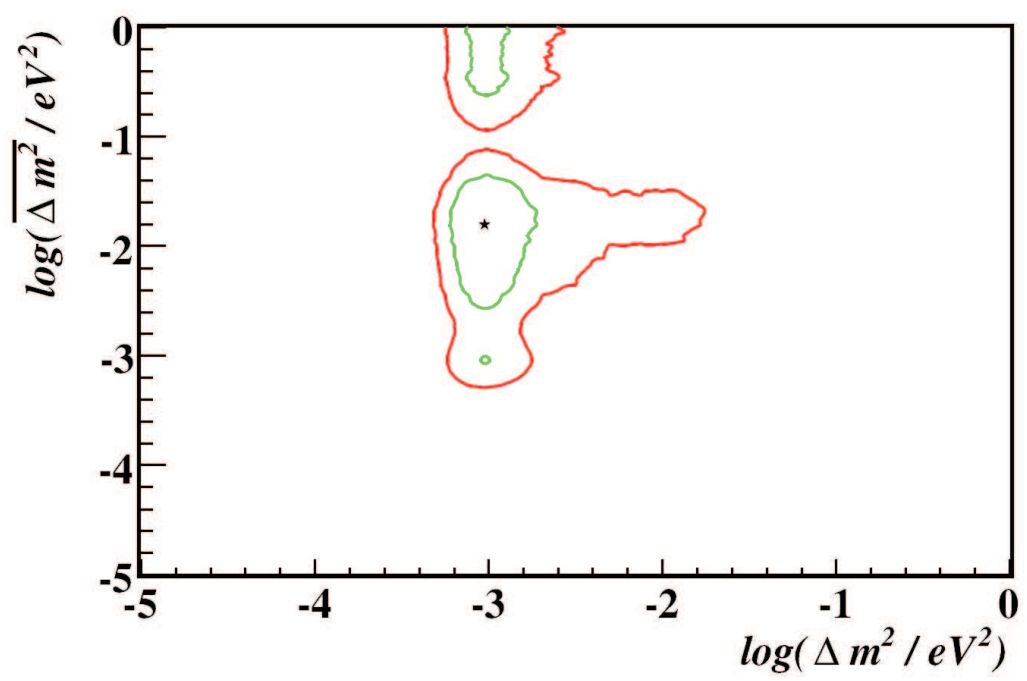

Figure 2.10: The limits on a difference between $\Delta m_{\text {atm }}^{2}$ and $\Delta \bar{m}_{\text {atm }}^{2}$ set by analysis of atmospheric neutrinos in the MINOS far detector. The red line is the $90 \%$ c.l., the green line the $68 \%$ c.l.. Figure taken from [123].

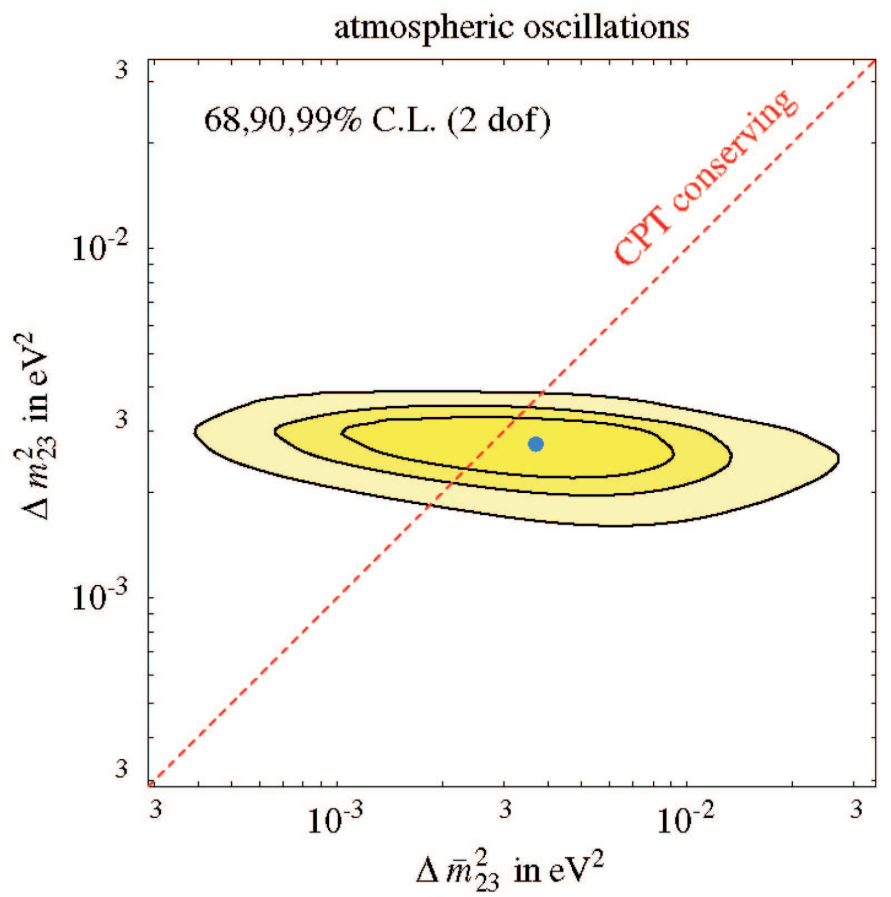

(a)

Figure 2.11: The combined world limits on differences between $\Delta m_{\mathrm{atm}}^{2}$ and $\Delta \bar{m}_{\mathrm{atm}}^{2}$. The figure is taken from [124]. 


\subsection{Looking for $\mathcal{C P} \mathcal{T}$ Violation with MINOS}

MINOS is a $\nu_{\mu}$ disappearance experiment. In such an experiment the action of $\mathcal{T}$ leaves the physical situation unchanged:

$$
\mathcal{T}\left(\nu_{\mu} \rightarrow \nu_{\mu}\right)=\left(\nu_{\mu} \rightarrow \nu_{\mu}\right)
$$

The mathematical formulism describing the two situations is therefore identical. In order for $\mathcal{C P} \mathcal{T}$ to be conserved $\mathcal{C P}$ violation cannot manifest itself, as $\mathcal{T}$ violation has no effect so cannot re-establish any symmetries broken by $\mathcal{C P}$ :

$$
\begin{aligned}
\mathcal{C P} \mathcal{T}\left(\nu_{\mu, L} \rightarrow \nu_{\mu, L}\right) & =\mathcal{C P}\left(\nu_{\mu, L} \rightarrow \nu_{\mu, L}\right) \\
& =\mathcal{C}\left(\nu_{\mu, R} \rightarrow \nu_{\mu, R}\right) \\
& =\left(\bar{\nu}_{\mu, R} \rightarrow \bar{\nu}_{\mu, R}\right) .
\end{aligned}
$$

Hence any observation of difference in the disappearance rates

$$
P\left(\nu_{\mu} \rightarrow \nu_{\mu}\right) \neq P\left(\bar{\nu}_{\mu} \rightarrow \bar{\nu}_{\mu}\right)
$$

appearing, at first sight, to be merely evidence of $\mathcal{C P}$ violation would, in fact, be direct evidence of $\mathcal{C P} \mathcal{T}$ violation. This measurement will be made in this thesis.

\subsection{Sterile Neutrinos}

The measurement of the $Z$ width (section 2.6) showed that only three flavours of light neutrino exist which couple through the weak neutral current. However the possibility remains that there is a fourth (or even more) flavour of sterile neutrino, which feels no weak interactions. This would be visible through its effect on the oscillations of the three active neutrino types: the PMNS matrix can be generalised 
to any number of generations and the logic of section 2.7.1 follows accordingly.

Results implying a fourth set of neutrino flavour and mass states were obtained by the LSND collaboration in the late 1990s [125] and the corresponding oscillation parameter space is shown in figure 2.3. They come from an observation of $\bar{\nu}_{\mu} \rightarrow \bar{\nu}_{e}$ appearance and are consistent with a fourth mass splitting in the region $0.2-10 \mathrm{eV}^{2}$.

However, a number of other experiments have ruled out much of the parameter space favoured by LSND (again shown in figure 2.3), most notably MiniBooNE [126] who have ruled out a large area of the LSND region. Yet MiniBooNE looked for $\nu_{\mu} \rightarrow \nu_{e}$ transitions, meaning the LSND signal could be explained through a $\mathcal{C P}$ violating effect (particularly if more than one sterile neutrino is introduced). MiniBooNE plans to release the corresponding antineutrino results in the next few years. Additionally, MiniBooNE observed an excess of $\nu_{e}$-like events at low energies (outside the LSND region) which is not yet explained (it could be a systematic effect, such as an unexpectedly high background from $\pi^{0}$ decays). The recently proposed MicroBooNE experiment [127] aims to investigate this region using liquid Argon technology.

Without a direct observation of $\nu_{\tau}$ appearance, atmospheric neutrino oscillations could involve a sterile component: $\nu_{\mu} \rightarrow \nu_{\mathrm{s}}$. The Super-Kamiokande collaboration have disfavoured pure $\nu_{\mu} \rightarrow \nu_{\mathrm{s}}$ transitions [128, 108], and placed a limit on a fractional sterile component in the $\nu_{\mu}$ disappearance process [129]: expressed through the sterile mixing angle $\zeta$, the limit is $\sin ^{2} \zeta<0.19$ (90\% c.l.).

MINOS has also produced limit on sterile neutrinos [130], making a comparison of the observed spectrum of neutral current (NC) events to that expected over the $735 \mathrm{~km}$ baseline, as shown in figure 2.12. Below a measured ${ }^{3}$ energy of $3 \mathrm{GeV}$ a

\footnotetext{
${ }^{3}$ This measured energy has a low correlation with the true incoming neutrino energy as, in these NC interactions, the outgoing neutrino can carry away a significant fraction of the event energy which is never detected. The various event topologies observed in the MINOS detectors are discussed in more detail in section 3.8.1.
} 


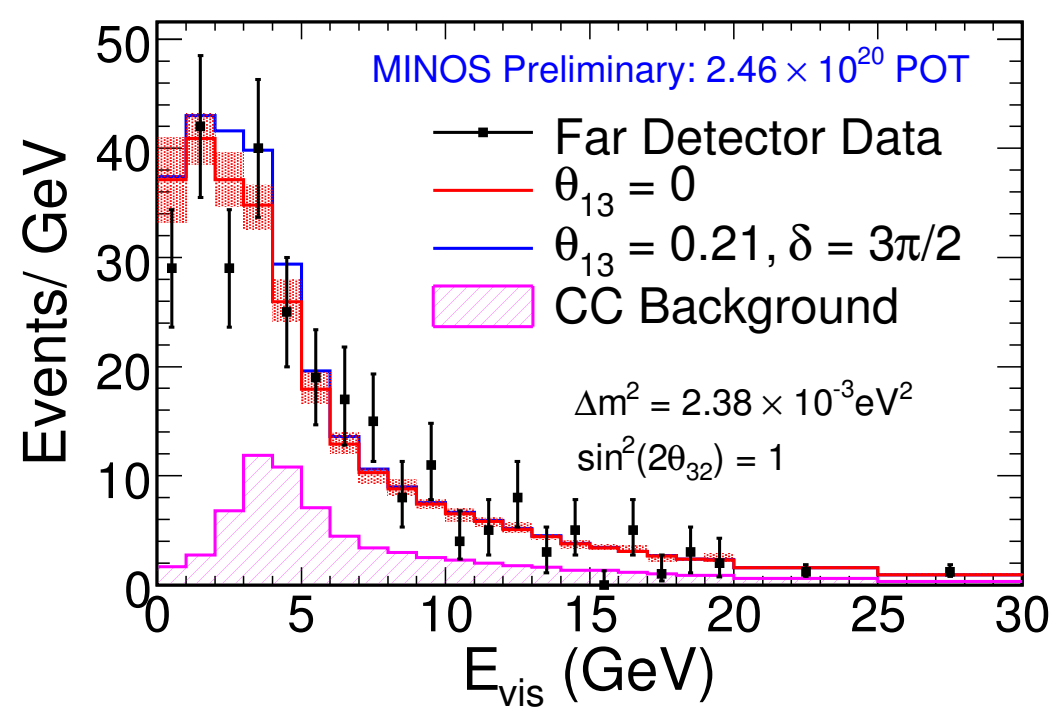

Figure 2.12: The spectrum of neutral current events (see section 3.8.1) observed at the MINOS far detector (black), with the expectation in the case of no sterile neutrino flavours assuming $\theta_{13}=0$ (red) and $\theta_{13}$ at the Chooz upper limit (blue). The red shaded area shows the systematic error on the prediction. The expected charged current background is shown in pink.

small deficit at the level of $1.15 \sigma$ is observed. This can be interpreted in terms of oscillations to sterile neutrinos, which would cause a deficit in the NC interaction rate as sterile neutrinos do not interact through the weak neutral current. Such an interpretation can be quantified as a disappearance fraction, $f$, of these events compared with the expectation:

$$
f=0.15_{-0.13}^{+0.12}
$$

This statistically limited result gives no conclusive indication of a sterile neutrino component; further measurements will be made as the exposure increases.

\subsection{Absolute Neutrino Mass}

Neutrino oscillation experiments have given values for the squared mass differences between mass states, but cannot say anything about the absolute value of neutrino 
masses. The best limits on this come from looking at the shape of the endpoint of the electron energy spectrum from tritium $\beta$-decay (similar to Fermi's original estimate of neutrino mass $[16,17])$. As these experiments look at electron neutrinos they do not measure directly a mass state and instead measure a combination of the mass states: $m_{\beta}^{2}=\sum_{i}\left|U_{e i}\right|^{2} m_{\nu_{i}}^{2}$ (in the tritium case corresponding to the antineutrino). The current world average limit is $m_{\beta}<2 \mathrm{eV}$ at $90 \%$ c.l. [58] (antineutrinos).

\subsection{Majorana or Dirac Neutrinos?}

Another question is the nature of the neutrino-antineutrino relationship. In the standard model, all particles are Dirac particles: the particle and antiparticle are distinct entities. This gives no explanation of the lack of coupling of the weak interaction to right handed neutrinos (and left handed antineutrinos). However a neutral fermion may also be a Majorana particle, in which case there is no distinction between particle and antiparticle. In this model, the right handed neutrino is in fact what we interpret as the right handed antineutrino, and thus does couple to the weak interaction.

This leaves open the possibility for a neutrinoless double $\beta$-decay process

$$
n n \rightarrow e^{-} p\left(\bar{\nu}_{e} \rightarrow \nu_{e}\right) n \rightarrow e^{-} p e^{-} p
$$

This process has not yet been observed, except for one positive claim [131] which is yet to gain wide acceptance in the neutrino physics community.

\section{$2.18 \nu_{\mu} \rightarrow \bar{\nu}_{\mu}$ Transitions in MINOS}

A second process looked for in this thesis is that of $\nu_{\mu} \rightarrow \bar{\nu}_{\mu}$ transitions. The process is similar to Pontecorvo's original oscillation model $[61,62]$ but the lepton 
number violation required by that theory has been ruled out. There is no positive experimental evidence for this process, and no standard theoretical models which suggest it should happen. The $\nu_{\mu}, \bar{\nu}_{\mu}$ equivalence permitted by the Majorana theory does not alone allow this process as a spin-flip is required for a neutrino to exhibit its antineutrino characteristics; the neutrino mass is too small for this spin-flip to occur at an observable level. Through whatever process the neutrino becomes an antineutrino, a $V+A$ component of the weak charged current would additionally be required for the resulting left-handed $\bar{\nu}_{\mu}$ to interact.

Limits have been placed on $\nu \rightarrow \bar{\nu}$ transitions by previous experiments, through different channels and in different regions of oscillation parameter space from those investigated in this thesis. In 1982 limits were set, using the BEBC bubble chamber in the CERN SPS neutrino beam, of $\nu_{\mu} \rightarrow \bar{\nu}_{e}$ and $\nu_{e} \rightarrow \bar{\nu}_{e}$ transitions [132] through searches for $\bar{\nu}_{e}$ appearance. Working with neutrino energies starting from $10 \mathrm{GeV}$, with a mean $\nu_{\mu}$ energy of $46 \mathrm{GeV}$, and a baseline of $825 \mathrm{~m}$, limits were set of $P\left(\nu_{e} \rightarrow \bar{\nu}_{e}\right)<3 \times 10^{-2}$ and $P\left(\nu_{\mu} \rightarrow \bar{\nu}_{e}\right)<5 \times 10^{-4}$ (both at $90 \%$ c.l.).

In 1993, the E645 experiment at the Los Alamos Meson Physics Facility (LAMPF) placed limits on $\bar{\nu}_{e}$ appearance from a beam consisting of $\nu_{\mu}, \bar{\nu}_{\mu}$ and $\nu_{e}$ of energies below $55 \mathrm{MeV}$ with a baseline of $26.6 \mathrm{~m}$ [133]. They detected $8.3 \pm 3.4$ $\bar{\nu}_{e}$ events, consistent with the expected non-oscillation, non-transition background of $5.2 \pm 0.5$ events. In the case of $100 \% \nu_{\mu} \rightarrow \bar{\nu}_{e}$ transitions, $925.0 \pm 83.0 \bar{\nu}_{e}$ events were expected; for $100 \% \nu_{e} \rightarrow \bar{\nu}_{e}$ transitions, $587.0 \pm 55.0 \bar{\nu}_{e}$ events were expected.

MINOS has already measured the spectrum of $\nu_{\mu}$ events which are missing, attributed to oscillations, after travelling $735 \mathrm{~km}$. It is these missing events which are the potential source of $\bar{\nu}_{\mu}$ appearance. These missing events fall primarily at energies below $10 \mathrm{GeV}$; therefore for this analysis a measurement of the event rate in this region will be made in comparison to the expectation in the case of $\mathcal{C P} \mathcal{T}$ conservation $\left(\Delta \bar{m}_{\mathrm{atm}}^{2}=\Delta m_{\mathrm{atm}}^{2}\right)$. 


\section{Chapter 3}

\section{The MINOS Experiment}

\subsection{Neutrino Physics with MINOS}

The MINOS experiment uses the world's most intense neutrino beam (the NuMI beam) to investigate the phenomenon of neutrino oscillations.

The beam of primarily muon neutrinos is produced at the Fermi National Accelerator Laboratory (FNAL) in Chicago, USA. $1 \mathrm{~km}$ downstream from the beginning of the neutrino production area is the MINOS near detector, which makes precise measurements of the neutrino beam before oscillations occur. $735 \mathrm{~km}$ downstream from the neutrino production area is the MINOS far detector, located in the Soudan Underground Laboratory, northern Minnesota. This measures the neutrino beam after oscillations have taken place; and by a comparison of the neutrino energy spectra at the two detectors a precision measurement of the neutrino oscillation parameters can be made.

\subsection{1 $\nu_{\mu}$ Disappearance}

The goal of the MINOS experiment is to measure the atmospheric neutrino mass difference, $\Delta m_{\text {atm }}^{2}$, to an accuracy of at least $10 \%$; and furthermore put limits on the atmospheric mixing angle $\sin ^{2}\left(2 \theta_{23}\right)$ (projected sensitivities were shown in 

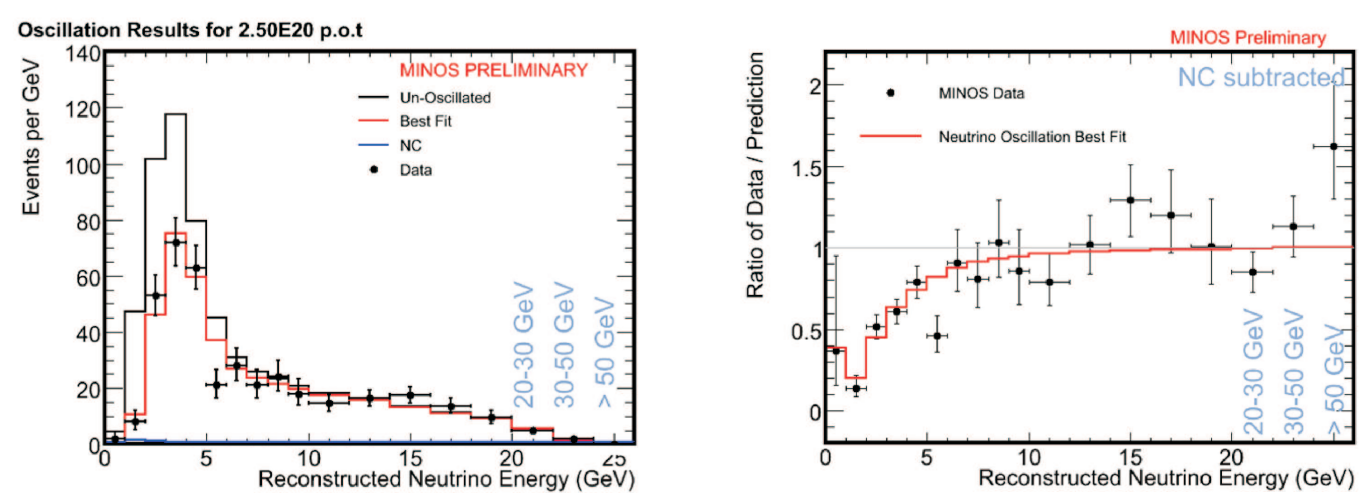

Figure 3.1: The most recently published MINOS $\nu_{\mu}$ disappearance measurement. The left graph shows the far detector $\nu_{\mu}$ energy spectrum. The black line is the predicted spectrum in the case of no oscillations. The black dots show the data. The red line is the prediction with oscillations applied, using oscillation parameters that give the best fit to the data. The right graph shows the ratio of the data to the unoscillated prediction (black dots) and the ratio of the prediction with best fit oscillations to the unoscillated prediction (red dots).

figure 2.8). This is done through the observation of $\nu_{\mu}$ disappearance.

Figure 3.1 shows the most recently published $\nu_{\mu}$ disappearance measurement made by MINOS. The measured near detector $\nu_{\mu}$ energy spectrum allows a predicted far detector spectrum to be calculated, in the case of no oscillations (black line). The $\nu_{\mu}$ spectrum at the far detector is measured (black dots) and a deficit in the number of events is observed. The far detector prediction can have oscillations applied, to obtain a best fit to the data (red line).

The connection between the MINOS data and the oscillation parameters is more clearly shown by the right hand plot in figure 3.1. This ratio of the measured $\nu_{\mu}$ far detector spectrum to the prediction with no oscillations is equivalent to a measurement of the $\nu_{\mu}$ survival probability $P\left(\nu_{\mu} \rightarrow \nu_{\mu}\right)$. The theoretical form of this probability from the two-flavour oscillation model (equation 2.4) is shown in figure 3.2. A characteristic dip is seen (here at $\sim 1.5 \mathrm{GeV}$ ), corresponding to that seen in the MINOS data. The depth of this dip is governed by $\sin ^{2}\left(2 \theta_{23}\right)$ and the energy at the dip by $\Delta m_{\mathrm{atm}}^{2}$. (Below the dip the energy resolution of MINOS is too low to resolve the individual oscillation dips so an averaged disappearance is 


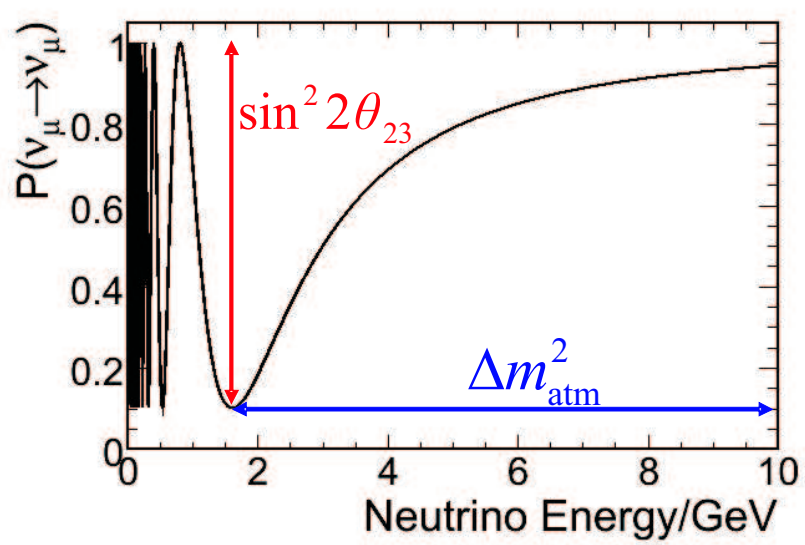

Figure 3.2: The $\nu_{\mu}$ survival probability in the two-flavour model (equation 2.4) as a function of neutrino energy. The oscillation parameter $\Delta m_{\mathrm{atm}}^{2}$ governs the position of the dip, and the parameter $\sin ^{2}\left(2 \theta_{23}\right)$ governs the depth of the dip.

observed; see equation 2.5.)

\subsubsection{A Two-Detector Experiment}

The two-detector arrangement allows the reduction of systematic effects. Many systematic uncertainties affect both detectors in similar ways (for example uncertainties in neutrino cross sections or in the NuMI beam spectrum). Use of the near detector to predict the far detector spectrum allows these effects to be corrected for in that prediction.

\subsubsection{Further Physics Potential of MINOS}

Other physics measurements accessible to MINOS have been discussed in chapter 2. A measurement of $\nu_{e}$ appearance at the far detector can give information about the mixing angle $\theta_{13}$ (section 2.8.3; see figure 2.9 for the MINOS sensitivity). Through an observation of neutral current neutrino interactions (see section 3.8.1) a limit can be put on the existence of sterile neutrinos (section 2.15 and figure 2.12). Measurements of the beam in the near detector can be used to make neutrino cross section measurements. 
This thesis will focus primarily on observations of antineutrinos relating to the NuMI beam. The MINOS detectors are magnetised, enabling neutrinos and antineutrinos to be separated through their CC interactions: the charge of the lepton produced (see figure 2.1) can be identified and used to tag the neutrino type. The beam contains a $6 \% \bar{\nu}_{\mu}$ background. An analysis similar to the $\nu_{\mu}$ disappearance analysis discussed above can be performed on these neutrinos to provide a measurement of the antineutrino oscillation parameters and a resulting limit on $\mathcal{C P} \mathcal{T}$ violation. A search for an appearance of antineutrinos at the far detector, above the beam background, can be made as a search for the process $\nu_{\mu} \rightarrow \bar{\nu}_{\mu}$

The detectors are also able to look at cosmic neutrinos, again separating neutrinos from antineutrinos using the magnetic field. A measurement of neutrino oscillation parameters from these has already been published [134] and the $\nu_{\mu} / \bar{\nu}_{\mu}$ separation potential used to provide a limit on $\mathcal{C P} \mathcal{T}$ violation $[135,123]$.

Observations of cosmic muons are also possible: for example a measurement of the $\mu^{+}$to $\mu^{-}$ratio has been made [136] along with measurements of seasonal variations in the cosmic muon rate [137].

\subsection{The NuMI Neutrino Beam}

The 0.4 MW NuMI beam uses protons at $120 \mathrm{GeV}$ from the FNAL Main Injector (MI). They are directed downwards by $3.3^{\circ}$ : directly towards the far detector. The protons strike a segmented, water-cooled, graphite target (consisting of 47 segments with a total thickness of 1.9 nuclear interaction lengths) producing a spray of hadrons. Prior to the target the proton beam passes through a graphite baffle, a $150 \mathrm{~cm}$-long rod with an $11 \mathrm{~mm}$ radius central aperture, which reduces (by $95.5 \%$ ) the intensity of any badly-focused protons in order to protect the downstream components. The NuMI beamline is illustrated in figure 3.3 and 


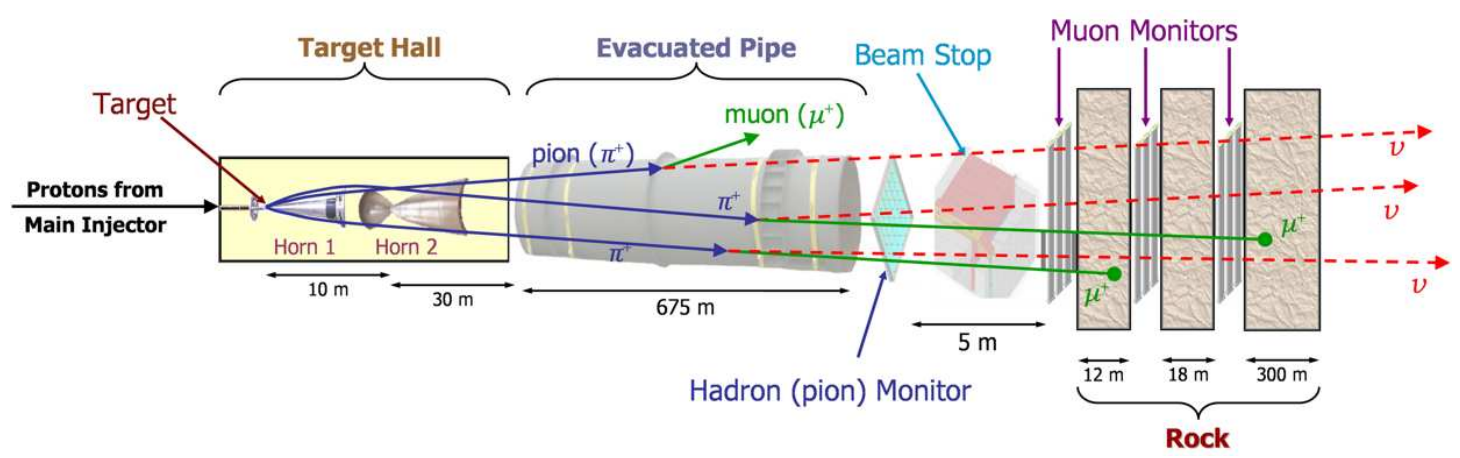

Figure 3.3: The components of the NuMI beamline. $120 \mathrm{GeV}$ protons from the FNAL Main Injector enter from the left hand side. Figure taken from [139].

more details can be found in [138, 139].

After the target the beam passes through two magnetic focusing horns (each producing a toroidal field about an axis in the beam direction), the first of which the target is mounted inside the mouth of, the second being $10 \mathrm{~m}$ downstream. These are magnetised in such a way as to focus mesons which will produce muon neutrinos: primarily positive pions and kaons through $\pi^{+} / K^{+} \rightarrow \mu^{+} \nu_{\mu}$. (It is possible to reverse the magnetic field to enable a $\bar{\nu}_{\mu}$ beam to be produced.) The focused mesons then travel down a $675 \mathrm{~m}$ decay pipe to allow the decay to neutrinos to take place. For the first two years of running (for all data analysed in this thesis), this decay pipe was evacuated (down to a few thousandths of an atmosphere). For future running the decay pipe will be filled with helium at close to atmospheric pressure as the strength of the upstream end of the decay pipe has been compromised by the acidic conditions created by the beam, raising concerns of implosion. After the decay pipe, an aluminium, steel and concrete beam absorber stops all remaining hadrons. There is then $300 \mathrm{~m}$ of rock before the near detector which is enough to stop any remaining particles (primarily muons) other than neutrinos.

The MI provides protons in a series of pulses or spills which can come as often as every $1.9 \mathrm{~s}$. These spills consist of six batches of protons; MINOS receives either five or six batches (the remaining batch going to produce antiprotons for 

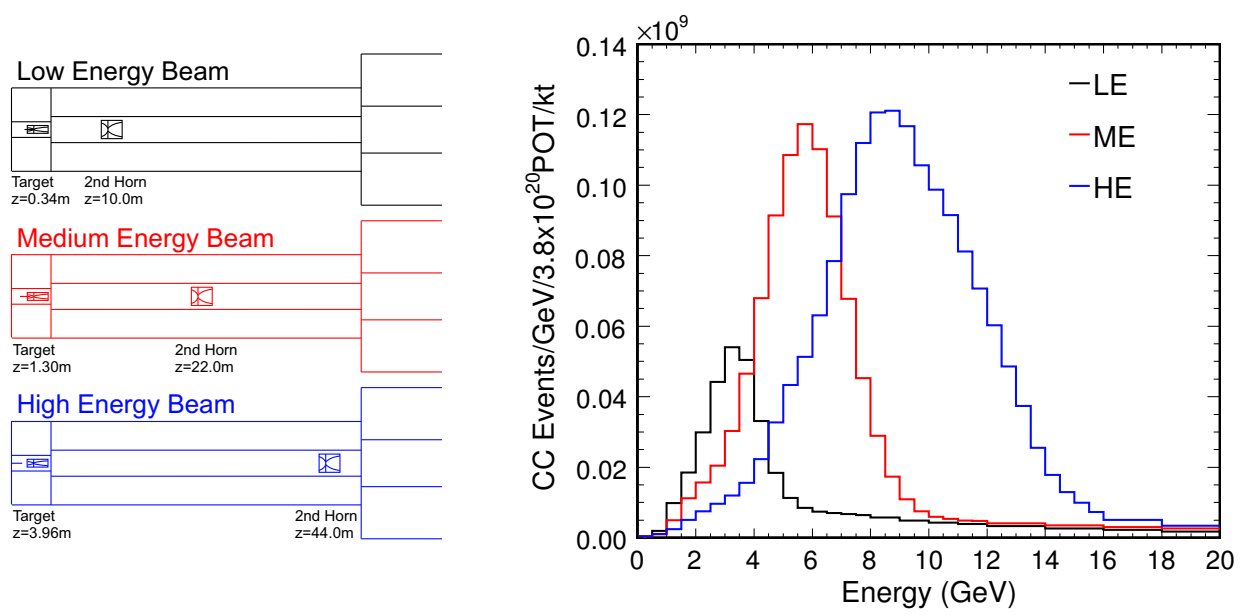

Figure 3.4: Three possible configurations of the NuMI beam (low, medium and high energy: LE, ME and HE). The relative target and focusing horn positions are shown on the left and the corresponding $\nu_{\mu}$ energy spectra on the right.

the Tevatron). The NuMI focusing horns are pulsed in coincidence with the arrival of these spills, reaching a peak magnetic field in the region of $3 \mathrm{~T}$. The typical number of protons in a six-batch spill is $2.5 \times 10^{13}$ (recent work has lead to the possibility that this will increase to around $4 \times 10^{13}$ ).

The neutrino energy spectrum provided by the NuMI beam is tunable, through changing the relative positions of targets and horns. Three of these configurations and their energy spectra are shown in figure 3.4. The main aim of MINOS is to measure accurately the position of the oscillation dip of figure 3.2. This dip falls in the region of $2 \mathrm{GeV}$, thus the low energy (LE) configuration of figure 3.4 has been chosen to maximise statistics in this region. The configuration actually used is a variant on this LE configuration. Moving the target $10 \mathrm{~cm}$ further upstream and running the horns at a lower current (185 kA rather than $200 \mathrm{kA}$ ) was found to give a higher flux in the energy region of interest. This is known as the $L E-10$ configuration and was used to obtain the data analysed in this thesis.

The composition of the LE-10 beam is shown in figure 3.5 (in terms of the number of $\mathrm{CC}$ interactions in the near detector). There is a background of $6 \%$ $\bar{\nu}_{\mu}$ events and $0.4 \%\left(\nu_{e}+\bar{\nu}_{e}\right)$ events. The $\bar{\nu}_{\mu}$ events arise mainly from the decay 


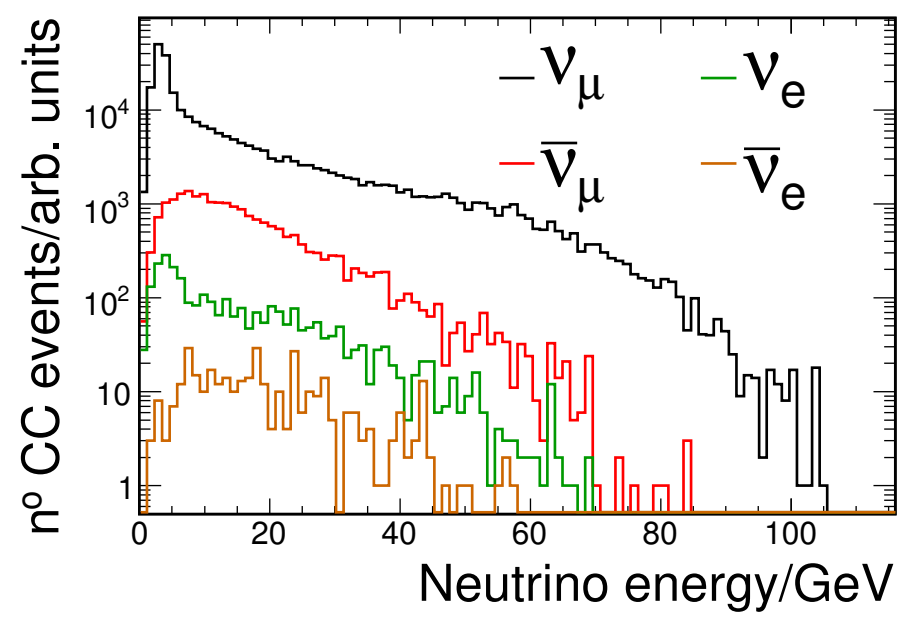

Figure 3.5: The spectra of CC neutrino interactions in the near detector in the LE-10 beam configuration arising from the various neutrino flavours in the beam.

of wrong-sign pions and kaons which pass straight along the axis of the horns, allowing little focusing. This effect is bigger at higher energies as the horns cannot as easily focus the high-energy hadrons. A small contribution also comes from muon decay.

\subsection{The MINOS Detectors}

\subsubsection{Overview}

For reasons discussed in section 3.1.2, the two MINOS detectors are designed to be as functionally similar as possible. Both detectors are steel-scintillator calorimeters, consisting of alternate layers of steel and scintillator. Detailed accounts of the detectors can be found in [140].

The near detector lies $110 \mathrm{~m}$ below ground. The neutrino beam at this point is intense enough that each proton spill yields of the order ten neutrino interactions in the detector's 980 tonnes. The far detector is located $705 \mathrm{~m}$ underground and sees only about of three neutrino interactions per day in the much larger detector mass of 5, 400 tonnes. The detectors are shown in figure 3.6. 

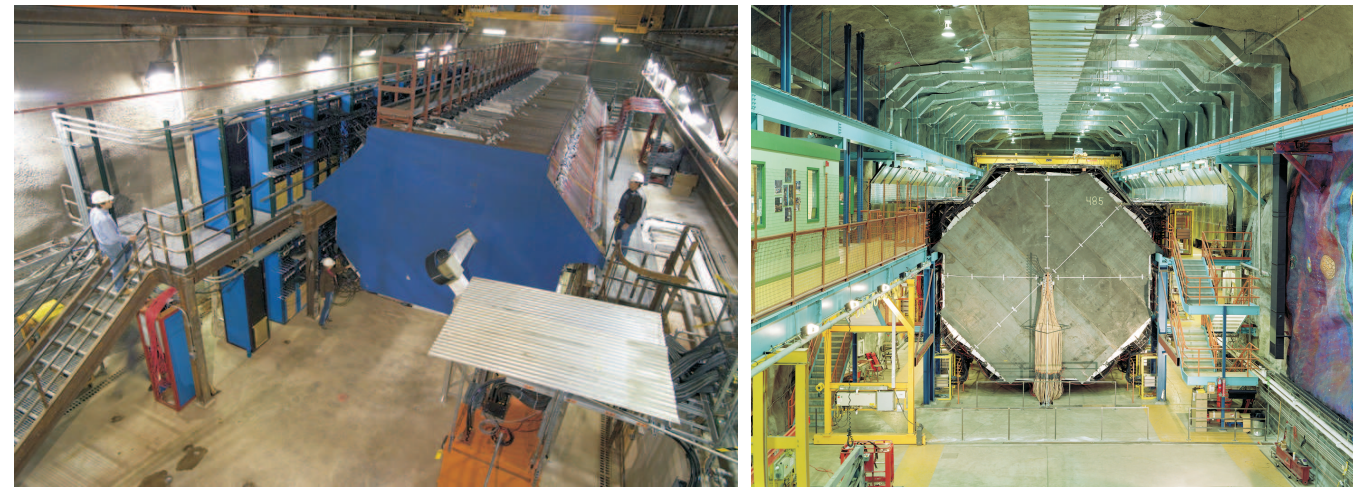

Figure 3.6: The MINOS near (left) and far (right) detectors.

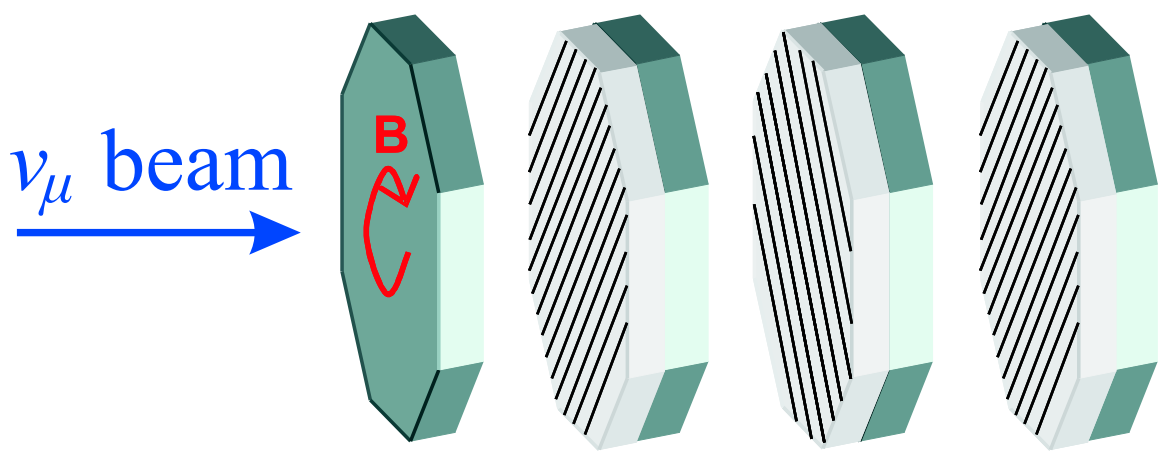

Figure 3.7: A schematic of a MINOS detector showing the scintillator strips and the darker steel planes.

\section{Far Detector}

The far detector is an octagonal prism of width $8 \mathrm{~m}$, consisting of 484 octagonal steel planes, alternated with planes of scintillator strips (figure 3.7). The scintillator strips are mounted on the upstream face of each steel strip. The far detector is split into two supermodules of 249 and 237 planes, separated by an air gap of $\sim 1.2 \mathrm{~m}$. No scintillator is mounted on the front plane of each supermodule. The steel planes are $2.56 \mathrm{~cm}$ thick, the scintillator planes $1 \mathrm{~cm}$ thick. An air gap takes the total plane separation up to $5.95 \mathrm{~cm}$. Running through a hole along the centre of the detector are coils of current-carrying cable (looping back around the outside of the detector) to create a cylindrical magnetic field of $\sim 1.3 \mathrm{~T}$ in the direction shown in figure 3.7 .

The scintillator planes are made up of $4 \mathrm{~cm}$-wide strips. The strips on adjacent 
planes are oriented perpendicular to each other (see figure 3.7) to allow threedimensional reconstruction of events.

The planes are oriented at $45^{\circ}$ to the vertical, defining a co-ordinate system referred to as the $u$ and $v$ directions. An alternative co-ordinate system defines the horizontal direction as $x$ and the vertical direction as $y$ (this latter system will be primarily used in this thesis). The beam direction is always labelled $z$.

The detector was designed to study muons produced in $\mathrm{CC} \nu_{\mu}$ interactions, hence the steel thickness chosen. A $2 \mathrm{GeV}$ muon will travel of the order of $3 \mathrm{~m}$ or 50 planes through the detector. The octagonal shape was chosen to most closely resemble a cylinder within a design involving easy to manufacture steel sheets.

\section{Near Detector}

Whilst maintaining the same pattern and thickness of steel and scintillator sheets, the near detector differs from the far detector in ways allowed by its proximity to the beam production point. The high interaction rate means much less detector is required. The shape is that of a squashed octagon ( $4.8 \mathrm{~m}$ by $3.8 \mathrm{~m}$, see figure 3.8$)$, the coil hole off-centre. The beam axis is displaced $1.48 \mathrm{~m}$ horizontally from the coil, in a region where the magnetic field is similar to that in the far detector, so as to limit the number of events depositing energy in the hole.

The detector consists of 282 steel planes. All of the front 120 planes (except the first) have scintillator mounted on them. Of these, every fifth plane is fully covered with scintillator; the rest have only a smaller region around the beam axis covered (see figure 3.8). Of the remaining planes, every fifth plane is fully covered with scintillator, the rest having no scintillator at all.

The detector is designed such that the front, highly instrumented part is to be used for hadronic shower energy measurements, whilst the back, partially instrumented part, is purely for muon tracking. 


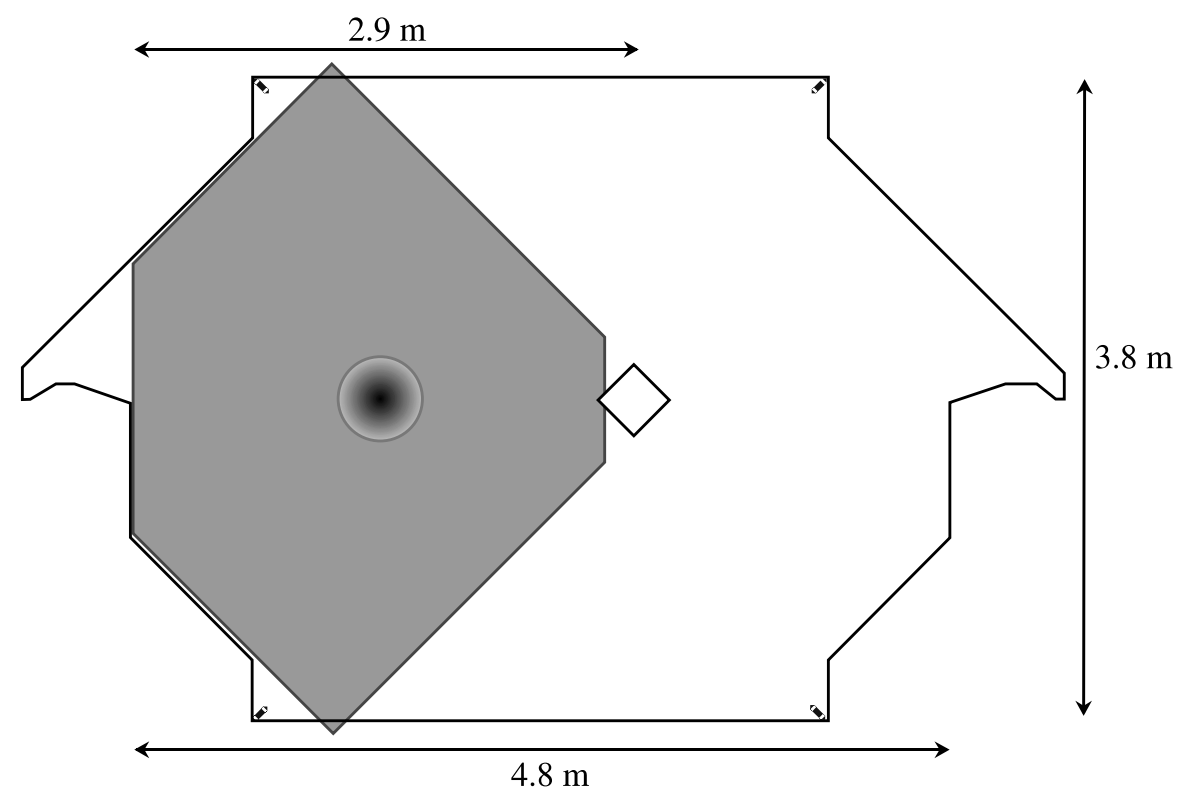

Figure 3.8: A near detector plane. The grey area is covered with scintillator in the partially instrumented planes, the entire plane being covered in the fully instrumented planes. The central diamond shows the position of the coil. The dark circle marks the centre of the neutrino beam.

\subsubsection{Steel}

The average steel density is $7.85 \pm 0.03 \mathrm{~g} \mathrm{~cm}^{-3}$ [140]. The typical RMS deviation of plane masses is $0.35 \%$ (the plane thickness varying by $0.3 \%$ ) [140].

\subsubsection{Scintillator}

The structure of a scintillator strip is shown in figure 3.9. The strips are made from extruded polystyrene, doped with organic scintillator (1\% by weight). They are coated with a reflective layer of $\mathrm{TiO}_{2}$ (15\% by weight). In a groove down one surface of each strip runs a $1.20_{-0.01}^{+0.02} \mathrm{~mm}$ diameter wavelength shifting (WLS) fibre. This shifts the scintillator light from blue to green (average wavelength $530 \mathrm{~nm})$ and transports it to the ends of the strips.

The scintillator strips are grouped into modules of between 13 and 28 strips, encased in $0.5 \mathrm{~mm}$ thick aluminium. The module arrangements are shown in figures 3.10 and 3.11 . 


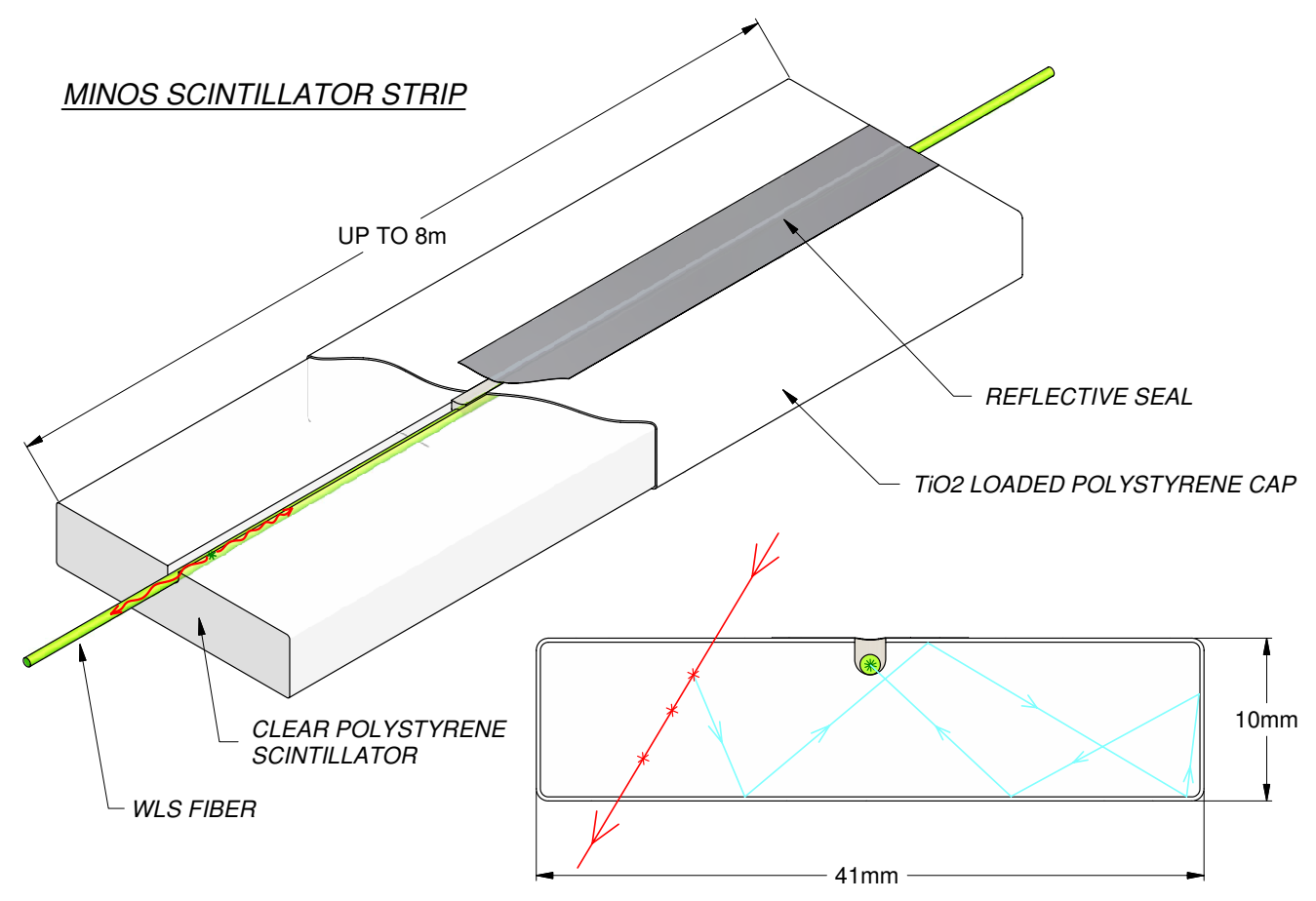

Figure 3.9: The structure of a scintillator strip.
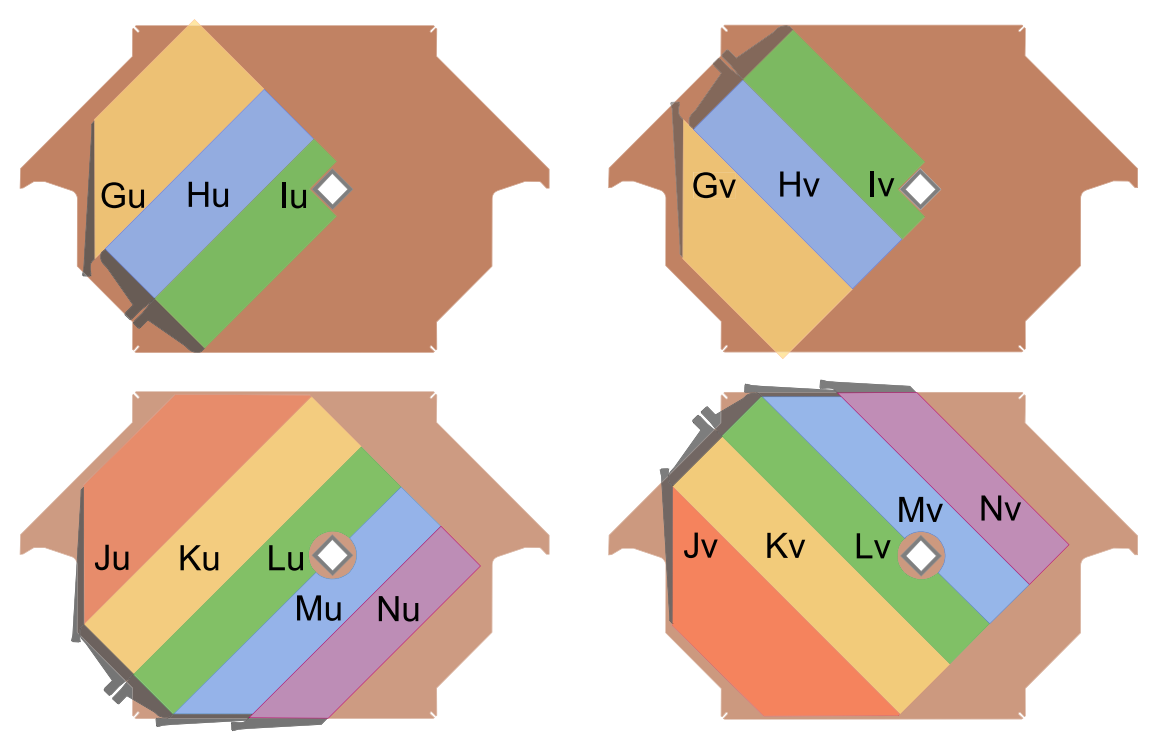

Figure 3.10: The scintillator module arrangement on a near detector plane. 


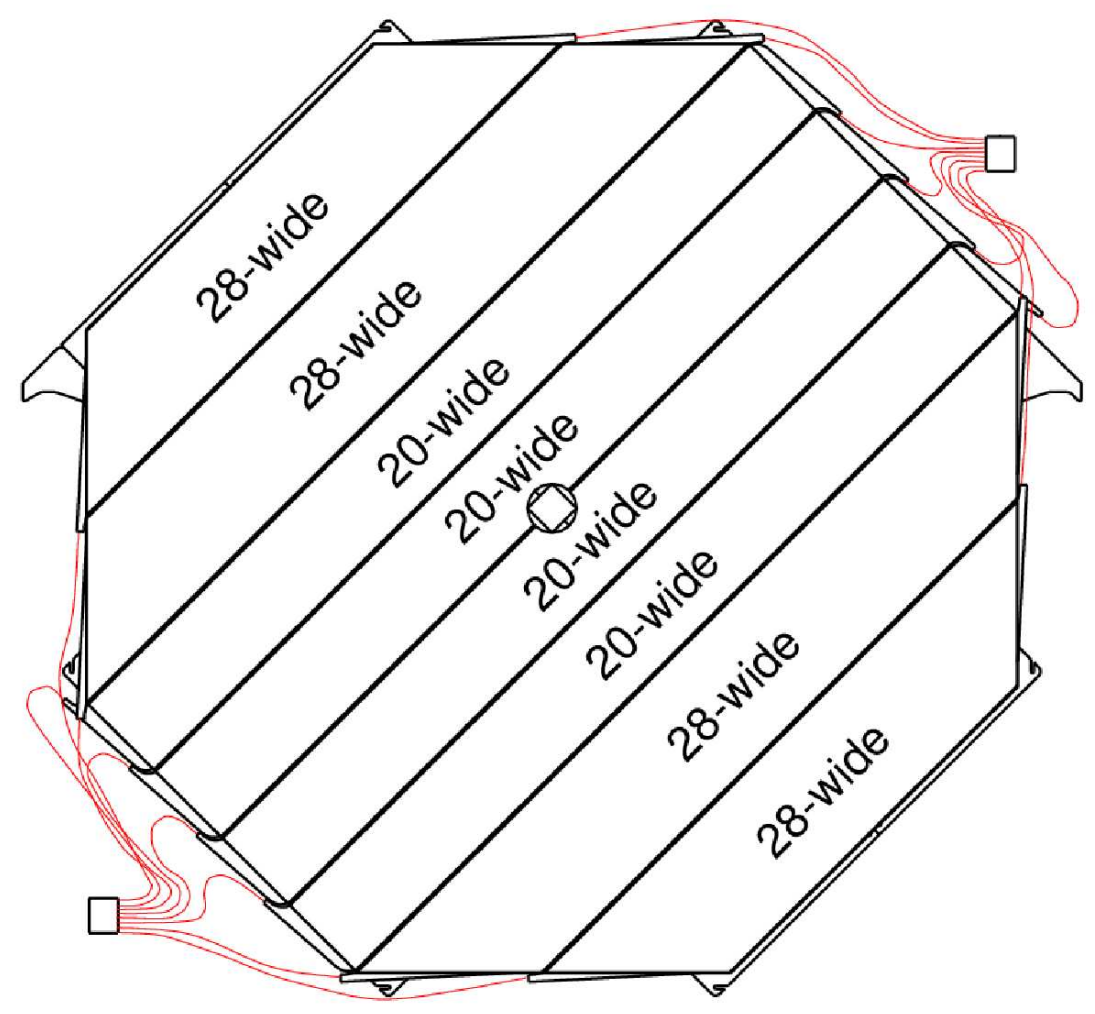

Figure 3.11: The scintillator module arrangement on a far detector plane. 


\subsubsection{Photomultiplier Tubes}

Light is carried from the WLS fibres, via clear fibres, to Hamamatsu multi-anode photomultiplier tubes (PMTs). At the far detector 16-anode PMTs are used (with gains up to $1 \times 10^{6}$ ); 64-anode PMTs are used at the near detector (set at a typical gain of $\left.0.8 \times 10^{6}\right)$. The voltage across the PMTs required to achieve these gains is $\sim 800 \mathrm{~V}$. The PMTs are housed in steel crates which, as well as being light-tight, provide shielding from the magnetic field.

At the far detector, eight scintillator strips from a single detector plane are read out by each PMT anode. These strips are separated by about $1 \mathrm{~m}$. The resultant ambiguities are resolved during reconstruction of the data using information from adjacent planes to identify the actual strip hit. The pattern of strips connected to a single anode are different on successive planes to aid this disambiguation.

At the near detector, every scintillator strip in the first 120 planes is read out uniquely by one anode. In the remaining planes, four scintillator strips from a single plane, again separated by approximately $1 \mathrm{~m}$, are read out by a single anode.

The multi-anode design of the PMTs allows some charge to drift within the PMT, registering a signal on the wrong (nearest-neighbour) anode: referred to as cross talk. This causes a low pulse height hit (with a signal of a size generally below the mean amplitude corresponding to one photoelectron $(p e))$ to be attributed to a scintillator strip with no true activity.

\subsubsection{Electronics}

\section{Near Detector}

Each beam spill (lasting up to $10 \mu \mathrm{s}$ ) can produce on the order of 10 neutrino events in the near detector. In order to reconstruct all these events, the near detector electronics [141] is designed to be fast and have no deadtime. 
The shortest time required to be resolved is that between adjacent buckets of MI protons: the smallest discrete groups of protons forming the neutrino beam. The size of these buckets is defined by the frequency of the accelerator's RF cavities: $53 \mathrm{MHz}$. The near detector electronics is therefore designed to record signals with a deadtimeless granularity of 18.8 ns.

The signal from each PMT anode is fed into a QIE (Charge (Q) to Current (I) Encoder). In this QIE the signal first encounters a current splitter, which divides the current into eight ranges: a set of eight capacitors, each of which is sensitive to a different range of currents (increasing in a binary pattern). The capacitors of all ranges below that corresponding to the signal size are saturated, the current in the first unsaturated range providing the precision measurement of the signal. This system works to increase the dynamic range of the readout. The current splitter integrates all the current in a single $53 \mathrm{MHz}$ clock cycle, the first unsaturated range being selected in the next cycle. In the subsequent cycle this analogue signal is converted to an eight bit digital signal using an ADC (analogue to digital converter; not part of the QIE itself). A fourth clock cycle is required to reset the capacitors in the current splitter. To prevent the readout being dead for the three clock cycles following the integration stage four current splitters reside on each QIE, each integrating in turn for one cycle, all using the same range selector and ADC.

The eight bit ADC output is increased to a 13 bit word with the addition of bits to encode the analysed range of the current splitter, and which of the four current splitters were used. This word is received by a 1000 word FIFO (first in, first out) buffer. This FIFO is large enough to buffer all words in a $10 \mu$ s spill, with room to spare.

The QIE is mounted on a board called a MENU module (MINOS Electronics for Neutrinos), along with the ADC and FIFO. Also on this board is a DC current injector which can inject a signal into the QIE for calibration. 
16 MENU modules are mounted on a MINDER module (MINOS Near Detector Electronics Readout), which time-stamps the signals and controls the modes of the MENUs (e.g. initiating the calibration mode). The MINDER reads sequentially all words from all its FIFOs, sending them to a single FIFO on the next module in the chain: the MASTER module (MINOS Acquisition, Sparsifier and Timestamper for Event Readout).

Each MASTER module holds eight MINDERs. It reads the data from these MINDERs into two buffers, which are read out by a VME computer (one buffer is written to while the other is being read). The VME computers send the data to the data acquisition (DAQ) system.

\section{Far Detector}

The far detector electronics (discussed in [142]) is not as fast as that of the near detector as the low event rate means deadtime can be tolerated.

Each PMT is read out by a single chip, called a VA chip ${ }^{1} .22$ channels on these chips are used: 16 for reading out the 16 anodes of the PMT, one for reading out the PIN diode monitoring the light injection system (section 3.4), and five for performing noise subtraction.

A signal from each PMT is also sent to a chip called an ASDLite which produces a signal if the PMT has registered more than $\sim \frac{1}{3}$ of the mean amplitude corresponding to one photoelectron. This signal is sent to a VARC (VA Readout Controller), each of which controls 36 VA chips, and provides the lowest level trigger for readout of the VA chip to begin. It additionally provides the trigger for an ETC (Event Timestamp Controller) to timestamp the readout signal.

The signal is digitised by an ADC which is part of a VMM (VARC Mezzanine Module): a module intermediate between the VARC and VA chip. Each VMM (and therefore each ADC) is responsible for six VA chips, digitising each in se-

\footnotetext{
${ }^{1} \mathrm{~A}$ version of the Viking chip manufactured by IDEAS of Norway.
} 
quence. The digitisation of a single VA chip takes $\sim 5 \mu \mathrm{s}$; hence if all six need to be read out this takes a total of $\sim 30 \mu$ s, during which time all six chips are dead to new signals.

The VARCs are then read out by a VME computer as in the near detector, again with two buffers per VARC to allow simultaneous reading and writing.

\subsection{Light Injection}

For calibration of the PMTs each detector is equipped with a light injection system which allows varying intensity pulses of ultra-violet light from LEDs to be shone onto the WLS fibres at the point when they exit the scintillator strips, mimicking the light output from the scintillator. The intensity of the light pulses is monitored with PIN diodes which receive light directly from the LEDs and produce a signal shaped to be similar to that of a PMT, which can be read out with the standard detector electronics.

\subsection{Triggering}

When a beam spill occurs, a signal (spill trigger) is sent to the near and far detectors triggering detector readout for $100 \mu$ s around the spill. At the near detector the readout within this period is triggerless. However, the noise rate at the far detector is too high for data to be read in a triggerless manner: $\sim 5 \mathrm{kHz}$. This dark noise arises from the PMTs and WLS fibres. A low level '2/36' trigger is therefore applied during any readout period. As discussed in section 3.3.5, each VARC reads out up to 36 PMTs, the signal for readout to begin coming from the ASDLite chips. The 2/36 trigger works such that the VARCs only begin digitisation of the data if these ASDLite signals are received from two of its PMTs within 400 ns of each other; this is an ample threshold to reduce the dark noise 
rate to a satisfactory level.

Outside beam spills, offline triggers cause data from cosmic muons to be stored, basing the decision on either a minimum amount of energy deposited in a set of planes, or a certain number of planes hit within a group of planes.

\subsection{The Calibration Detector}

To look at the response of the MINOS detectors to known-energy particles of various types a calibration detector was set up in a number of test beams at CERN [143]. It consisted of 60 unmagnetised planes, each $1 \mathrm{~m}$ square, of the same steel and scintillator structure as the two main MINOS detectors (the steel planes being $0.4 \mathrm{~mm}$ thinner), with 24 scintillator strips per plane. To compare the differing near and far detector readout systems, one end of each calibration detector scintillator strip was read out with the near detector system, the other end with the far detector system. The detector was placed in beams consisting of $e, \mu, \pi$ and $p$, with energies between $0.6 \mathrm{GeV}$ and $10 \mathrm{GeV}$.

The responses of the near and far detector readout systems were found to be identical to within $0.6 \%$ [144]. Observations of muons in the detector allowed a systematic error to be placed on muon energies measured from range, as will be discussed in section 8.3. Observations of hadronic [145] and electromagnetic [146] showers allowed the calorimetric energy resolution in MINOS to be measured as $(55 \% / \sqrt{\text { energy }})$ for hadrons and $(20 \% / \sqrt{\text { energy }})$ for electrons, as shown in figure 3.12 . 


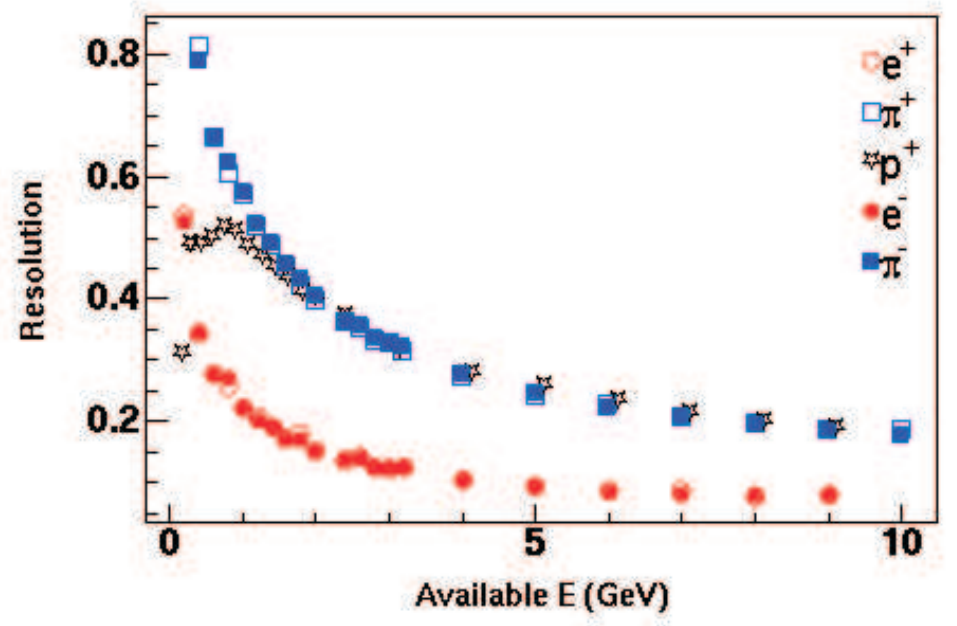

Figure 3.12: The calorimetric energy resolution, as a function of incident particle energy, of the MINOS detectors to hadronic and electromagnetic showers, as measured by the calibration detector.

\subsection{Simulation Software}

\subsubsection{Neutrino Beam}

The production of hadrons from protons incident on the NuMI target is simulated with the FLUKA package [147], the results of which are passed to the GEANT3[148]-based GNUMI framework which simulates the other components in the NuMI beamline. The decay kinematics of the hadrons are simulated, to produce a neutrino flux at both detectors. The spectrum of hadrons in the NuMI beamline produced by this simulation will be directly used in this thesis (see chapter 7).

At analysis time the fully simulated detected neutrino spectrum is reweighted according to a number of beamline parameters to better match the measured near detector spectrum, the same reweighting being applied to the far detector Monte Carlo. The simulated neutrinos (and antineutrinos) are reweighted according to the longitudinal and transverse momentum of their original parent hadrons in 
the NuMI target. To obtain the reweighting parameters, the longitudinal and transverse momentum distributions are parameterised and these parameters, along with a number of other beam and detector-related parameters, are altered until the simulated reconstructed neutrino energy spectrum in the near detector best matches that of the data. (The other beam and detector parameters used in the fit are not then used to reweight the data.) This latter set of beam and detector parameters include neutrino cross sections, detector hadronic energy response, and the current in the beam focusing horns. The horn current is supplied, through striplines, by banks of capacitors which pulse the horns at each beam spill. As the striplines warm up at the start of each period of running the horn current decreases. To cover this, a conservative 1\% uncertainty is assigned to the current.

\subsubsection{Detectors}

The neutrino interactions in the detectors are simulated with NEUGEN3 [149], using the MODBYRS-4 cross section model. This forms part of the GMINOS detector simulation framework which takes the particles produced by NEUGEN and passes them to a GEANT3-based detector model which propagates the particles through the geometry, taking into account physics processes the particles may undergo (using the GCALOR package [150]), down to an energy of $10 \mathrm{keV}$ (at which point all the remaining energy is deposited).

The simulated energy deposits are translated into scintillator light output and then to detector readout signals using two $\mathrm{C}++$-based MINOS-developed packages: Photon Transport simulating up to the photoelectrons produced at the PMT cathodes, DetSim dealing with the simulation thereafter. 


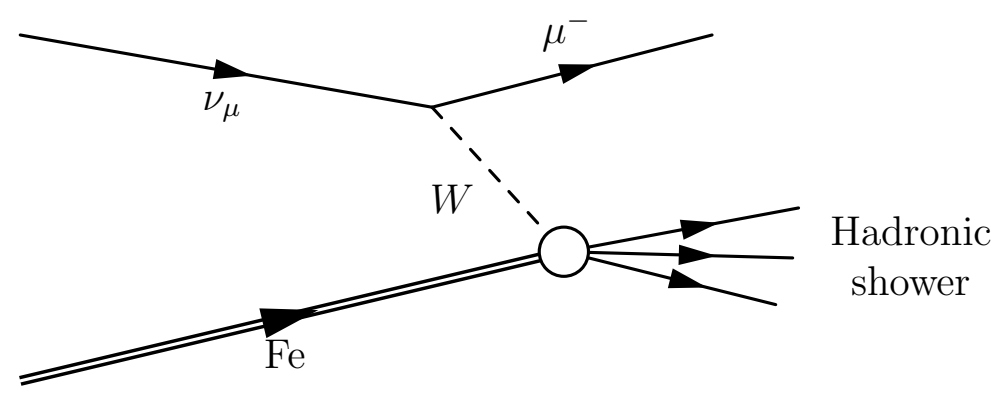

Figure 3.13: The charged current interaction of a $\nu_{\mu}$ with an iron nucleus.

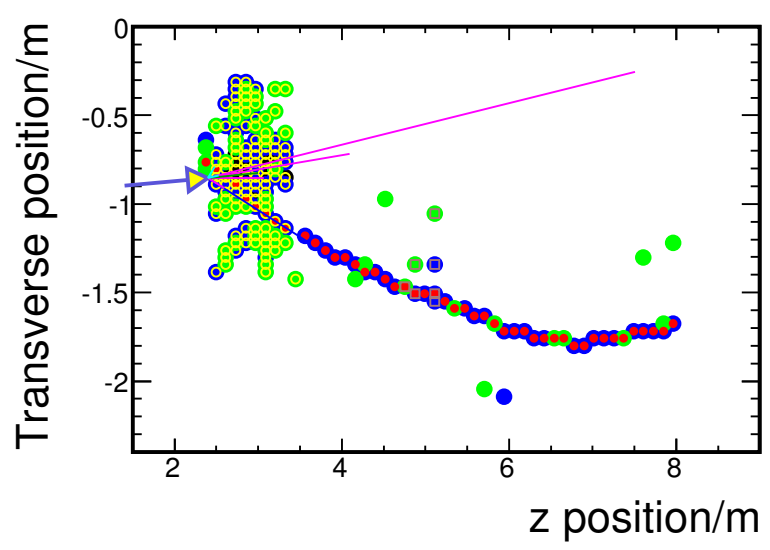

Detected energy depositions

- Deposition $<2.0$ pe

- $2.0<$ Deposition $<20.0$ pe

- Deposition $>20.0$ pe

Reconstruction

- Reconstructed track hit

Reconstructed shower hit

Monte Carlo Truth

$\underset{-\mu^{-}}{-\pi^{ \pm}}=\pi^{0}$

Figure 3.14: A typical high energy charged current $\nu_{\mu}$ event in the MINOS simulation. The incoming neutrino has an energy of $28.7 \mathrm{GeV}$; the muon carries away $3.7 \mathrm{GeV}$. The dots show the reconstructed positions of scintillator strips registering activity (green: low activity; blue: medium; black: high). Red circles indicate the activity has been associated with a reconstructed track, yellow circles with a reconstructed shower. The lines show the direction of the initial particle trajectories; the lengths of the lines are proportional to the initial particle energies.

\subsection{Neutrino Interactions in the MINOS Detec-}

\section{tors}

\subsubsection{Event Topologies}

The MINOS detectors are optimised for the identification and energy measurement of charged current $\nu_{\mu}$ interactions such as that shown in figure 3.13. The resulting pattern of energy depositions in a MINOS detector is shown in figure 3.14. The hadronic shower and $\mu^{-}$track are clearly visible. The magnetic field bends the 


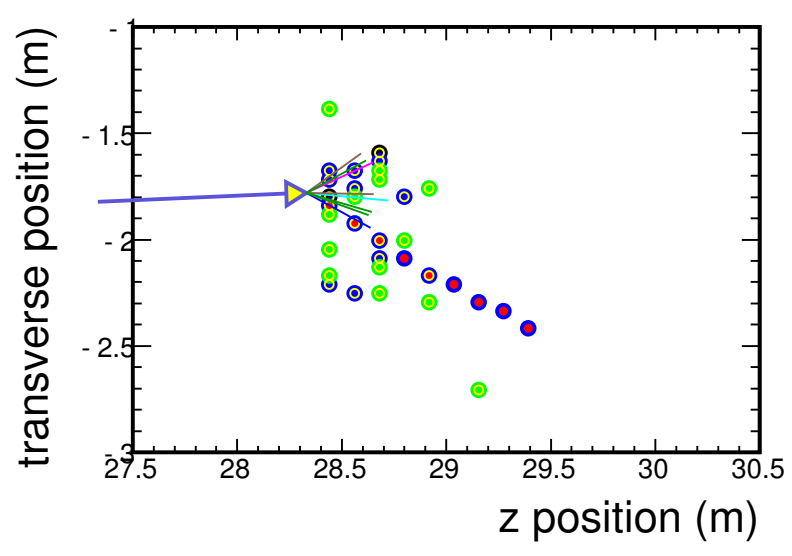

Detected energy depositions

- Deposition $<2.0$ pe

- $2.0<$ Deposition $<20.0$ pe

- Deposition $>20.0$ pe

Reconstruction

- Reconstructed track hit Reconstructed shower hit

Monte Carlo Truth

$\rightarrow$ Initial $v_{\mu}$

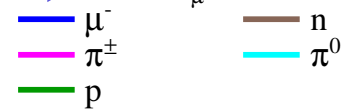

Figure 3.15: A typical low energy charged current $\nu_{\mu}$ event in the MINOS simulation. The incoming neutrino has an energy of $3.6 \mathrm{GeV}$; the muon carries away $1.0 \mathrm{GeV}$. See figure 3.14 for details of the event display.

muon trajectory, focusing negative muons towards the central axis of the detector.

The CC interaction of a $\bar{\nu}_{\mu}$ produces a positive muon, which is bent in the opposite direction by the magnetic field. It is this bending which is used to separate $\mathrm{CC} \nu_{\mu}$ and $\bar{\nu}_{\mu}$ interactions. However the $\mu^{+}$is focused outwards by the magnetic field and is therefore more likely to leave through the side of the detector. Thus less of the muon track is available for identification.

The interaction shown in figure 3.14 is of a high-energy neutrino: $28.7 \mathrm{GeV}$, although the $\mu^{-}$carries only $3.7 \mathrm{GeV} / c$ of momentum. The oscillation signal for which this analysis is searching will mostly affect neutrinos below $10 \mathrm{GeV}$. The $\mathrm{CC}$ interaction of a $3.6 \mathrm{GeV}$ neutrino in which the muon carries away $1.0 \mathrm{GeV}$ is shown in figure 3.15. With a much shorter muon track, the direction of curvature in the magnetic field is harder to identify; in particular, multiple or hard scattering of the muon can cause the overall curvature of the muon to be opposite to that caused by the magnetic field. Therefore at low energies, $\mathrm{CC} \nu_{\mu}$ interactions are an important background to a $\bar{\nu}_{\mu}$ analysis.

At higher energies $\mathrm{CC} \nu_{\mu}$ interactions can still be misidentified if a hard scatter such as in figure 3.16 causes a sharp bend in the track opposite to the direction 


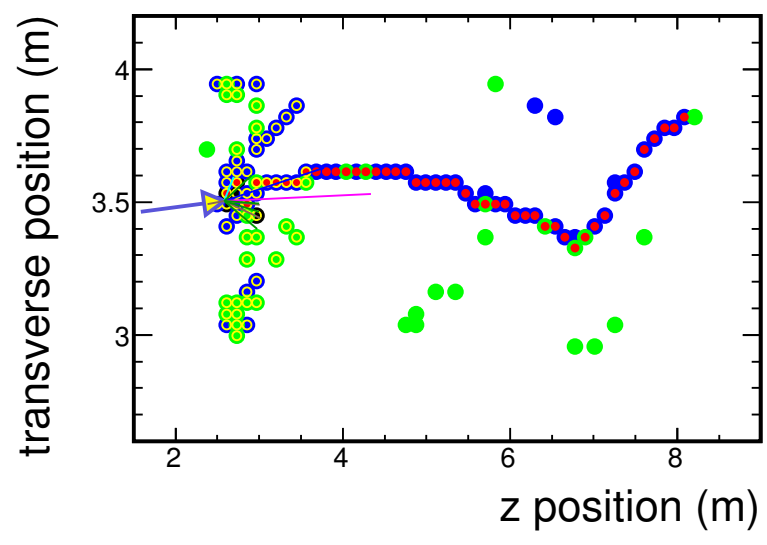

Detected energy depositions

- Deposition $<2.0$ pe

- $2.0<$ Deposition $<20.0$ pe

- Deposition $>20.0$ pe

Reconstruction

- Reconstructed track hit Reconstructed shower hit

Monte Carlo Truth

$\rightarrow$ Initial $v_{\mu}$

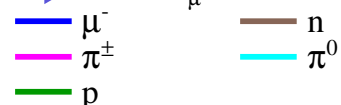

Figure 3.16: A high energy charged current $\nu_{\mu}$ event in the MINOS simulation in which the $\mu^{-}$undergoes a hard scatter. The incoming neutrino has an energy of $12.1 \mathrm{GeV}$; the muon carries away $3.8 \mathrm{GeV}$. See figure 3.14 for details of the event display.

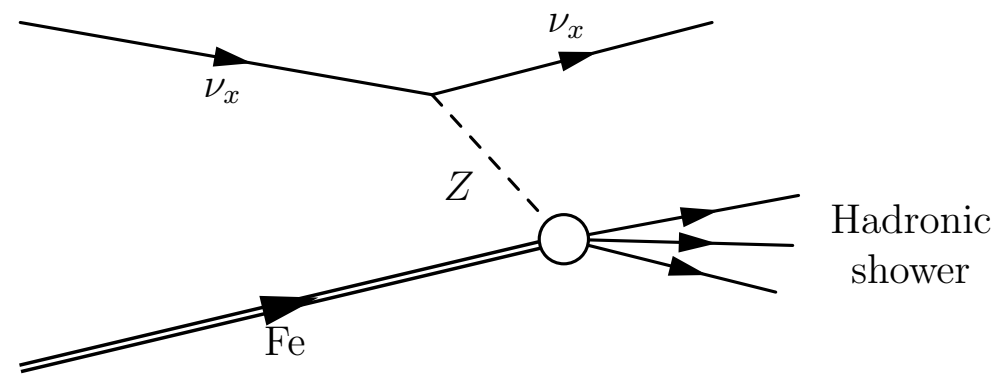

Figure 3.17: The neutral current interaction of any active neutrino flavour with an iron nucleus.

expected from the magnetic field. (This is less of a problem than at low energies as there is still a large amount of track bending in the expected direction.)

A further class of events, which must be eliminated in this analysis, is that of the NC interactions of all neutrino flavours, as illustrated in figure 3.17. The interaction differs from the charged current case (figure 3.13) in that a neutrino replaces the outgoing charged lepton. No muon track is therefore observed in the detector. A typical signature in the MINOS detectors is shown in figure 3.18. A diffuse hadronic shower with no reconstructed tracks is shown. This type of event is easily eliminated by requiring a track in a selected event. However, the hadronic shower can itself contain reconstructed tracks as in figure 3.19, and a hadron 


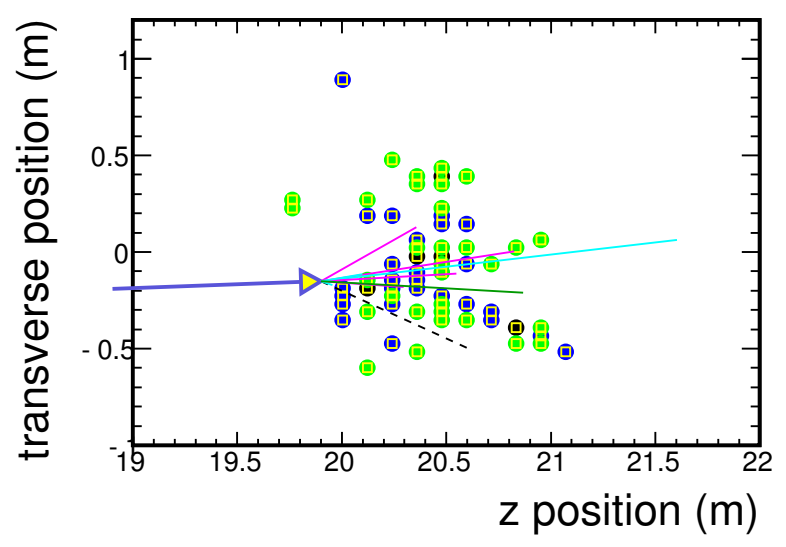

Detected energy depositions

- Deposition $<2.0$ pe

- $2.0<$ Deposition $<20.0$ pe

- Deposition $>20.0$ pe

Reconstruction

- Reconstructed track hit Reconstructed shower hit

Monte Carlo Truth

$\rightarrow$ Initial $v_{\mu} \quad \cdots$ Outgoing $v$

$\begin{array}{ll}\pi^{ \pm} & \mathrm{n} \\ \mathrm{p} & =\pi^{0}\end{array}$

Figure 3.18: A typical neutral current event in the MINOS simulation. The incoming neutrino has an energy of $17.9 \mathrm{GeV}$; the hadronic shower has $15.5 \mathrm{GeV}$ and the outgoing neutrino carries away $2.4 \mathrm{GeV}$. See figure 3.14 for details of the event display.

can itself produce a muon-like track such as the pion in figure 3.20 (particular problems being $\pi^{+}$tracks and, to a lesser extent, protons which can effectively imitate positive muons).

The final category of events is CC $\nu_{e}$ interactions (see figure 3.21 for an example). These consist of more compact, higher pulse height showers than the NC events (due to the electromagnetic component). In the antineutrino analysis, these are removed along with the NC events by cuts which aim to remove shower-like events.

\subsubsection{Interaction Processes and Cross Sections}

The neutrinos of interest to MINOS physics analyses have energies in the range $\mathcal{O}(1 \mathrm{GeV})-\mathcal{O}(100 \mathrm{GeV})$. Three main classes of interaction process dominate in this energy range: quasi-elastic, resonance and deep inelastic scattering.

Below $1 \mathrm{GeV}$ quasi-elastic interactions dominate:

$$
\nu_{\mu} n \rightarrow \mu^{-} p, \quad \bar{\nu}_{\mu} p \rightarrow \mu^{+} n .
$$




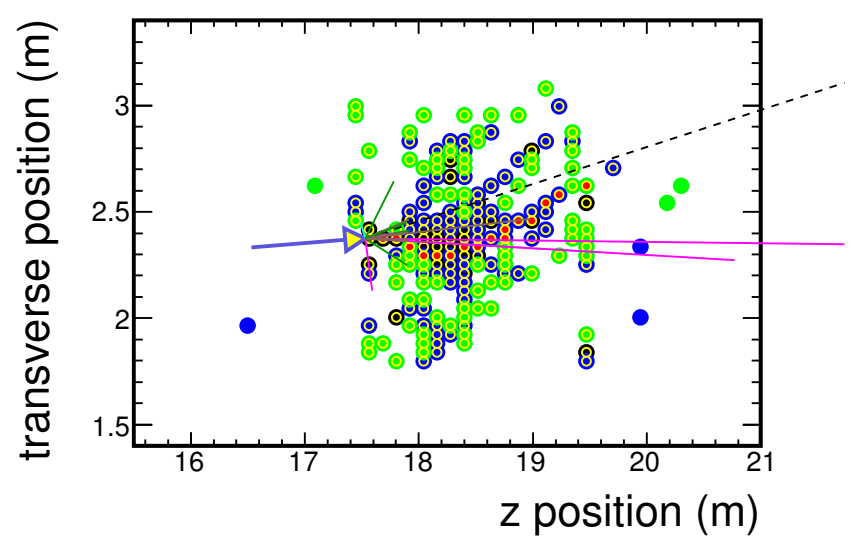

Detected energy depositions

- Deposition $<2.0$ pe

- $2.0<$ Deposition $<20.0$ pe

- Deposition $>20.0$ pe

Reconstruction

- Reconstructed track hit

Reconstructed shower hit

Monte Carlo Truth

$\rightarrow$ Initial $v_{\mu} \quad \ldots$ Outgoing $v$

$\pi^{ \pm}$
$\mathrm{p}_{\mathrm{K}}^{ \pm / 0}$

$\pi^{0}$

$\mathrm{K}^{ \pm 0}$

Figure 3.19: A neutral current event in the MINOS simulation in which a track has been reconstructed across the hadronic shower. See figure 3.14 for details of the event display.

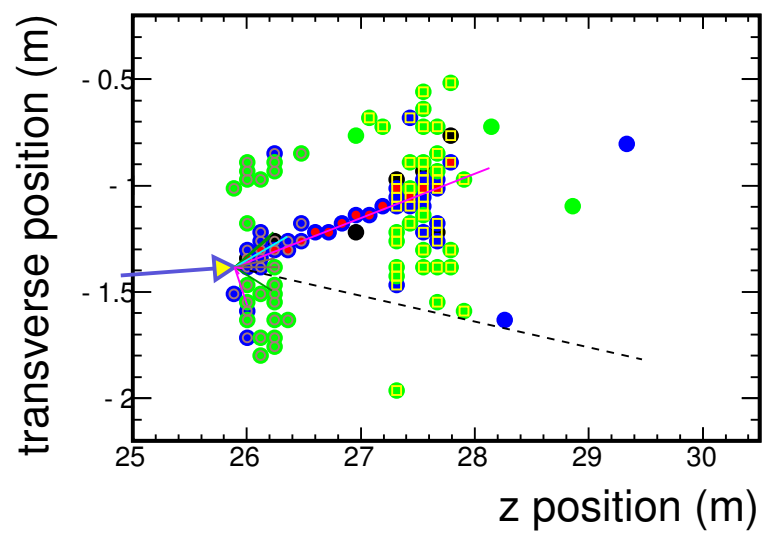

Detected energy depositions

- Deposition $<2.0$ pe

- $2.0<$ Deposition $<20.0$ pe

- Deposition $>20.0$ pe

Reconstruction

- Reconstructed track hit

Reconstructed shower hit

Monte Carlo Truth

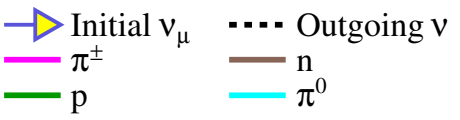

Figure 3.20: A neutral current event in the MINOS simulation which contains a true track from a hadronic particle. See figure 3.14 for details of the event display. 


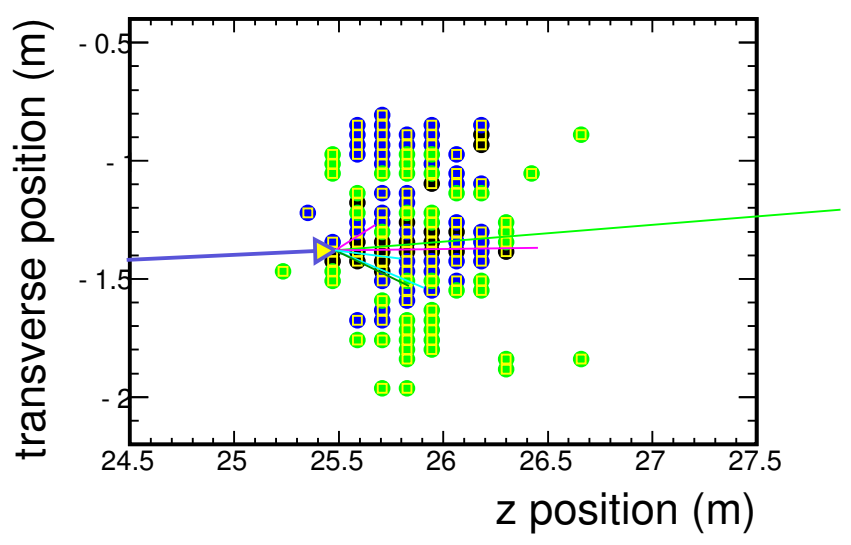

Detected energy depositions

- Deposition $<2.0$ pe

- $2.0<$ Deposition $<20.0$ pe

- Deposition $>20.0$ pe

Reconstruction

- Reconstructed track hit Reconstructed shower hit

Monte Carlo Truth

$\rightarrow$ Initial $v_{\mu}$

$=\mathrm{e}^{ \pm} \quad-\pi^{ \pm} \quad-\pi^{0}$

Figure 3.21: A charged current $\nu_{e}$ event in the MINOS simulation. See figure 3.14 for details of the event display.

Quasi-elastic neutrino cross sections are modelled using an approach set out by Llewellyn-Smith [151]. This uses time and charge symmetry considerations to simplify the form of the theoretical cross section. The remaining terms fall into two categories, describing either the vector $\left(\bar{\psi} \gamma^{\mu} \psi\right)$ or axial $\left(\bar{\psi} \gamma^{\mu} \gamma^{5} \psi\right)$ parts of the weak interaction. The vector terms can be well measured from electromagnetic interactions (this interaction being vector in nature). The axial terms can only be measured from neutrino interactions, so are less well known. The form factors are typically modelled with a dipole form

$$
\frac{1}{\left(1-\frac{q^{2}}{M_{V, A}}\right)^{2}}
$$

with $q$ the four-momentum transferred between the lepton and nucleus. $M_{V, A}$ is called the vector $(V)$ or axial $(A)$ mass, and is the only free parameter in this formulation. ${ }^{2}$ In MINOS, quasi-elastic interactions result in little or no hadronic activity, only the muon track typically being visible in the detector.

At a few $\mathrm{GeV}$, resonance production becomes an important interaction process. Here, the struck nucleus $(N)$ is excited to a baryon resonance $\left(N^{*}\right.$, the $\Delta(1232)$

\footnotetext{
${ }^{2}$ Work is underway in the MINOS near detector to make a measurement of the quasi-elastic $M_{A}$, as described in [152].
} 
dominating) which then decays, commonly to a nucleon-pion final state:

$$
\nu_{\mu} N \rightarrow \mu^{-} N^{*} ; \quad N^{*} \rightarrow \pi N^{\prime}
$$

Resonance interactions are modelled with the Rein Sehgal approach [153] which sums over all possible resonances, modelling each with a Breit-Wigner expression. The form factors are again modelled by the dipole form of equation 3.1. In MINOS the pion will be observed as well as the muon track. The pion can give a showerlike topology if it undergoes hadronic interactions, or else a track-like signature in the case of the $\pi^{ \pm}$. A pion track is very similar to that of a muon (the particles having similar masses), the $\pi^{+}$being a potential background in $\bar{\nu}_{\mu}$ identification. In the case of $\pi^{0}$ production, the decay $\pi^{0} \rightarrow \gamma \gamma$ produces an electromagnetic shower which is a significant background in a $\nu_{e}$ appearance search.

Above a few $\mathrm{GeV}$, the dominant process is deep inelastic scattering (DIS). Here the neutrinos probe inside the nucleons and scatter off the constituent quarks. The theoretical form of the cross sections is governed by structure functions describing the parton densities (valence and sea quarks, and gluons) inside the nucleons. A full treatment of DIS can be found in [154]. In MINOS, DIS produces events with a large hadronic shower, as illustrated in figure 3.14.

\subsubsection{Reconstruction Software}

The $\mathrm{C}++$-based reconstruction software aims to identify separately the detector hits resulting from hadronic showers and muon tracks, to associate them to the correct neutrino events (a non-trivial task in the high event-rate near detector), and finally to reconstruct the neutrino energy.

In the near detector, event timing is used to separate areas of detector activity within a single beam spill. A clustering algorithm looks for groups of hits to form hadronic showers, and tracks are found using an algorithm based on a Kalman fil- 
ter $[155,156,157]$. Spacial and timing considerations are further used to associate these tracks and showers into events. The hadronic energy estimate is made from the total signal from the clustered hits. The track energy, and also its direction of curvature, is calculated using the Kalman filter-based algorithm.

\subsubsection{Neutrino Energy Reconstruction}

In charged current $\nu_{\mu}$ and $\bar{\nu}_{\mu}$ interactions, the incoming neutrino energy is reconstructed by summing the energies of the reconstructed muon track and hadronic shower. If the muon track stops in the detector, its range is used to measure the initial energy. If the track exits, the energy is measured from the amount by which the muon bends in the magnetic field, the Kalman filter providing the measurement. The hadronic energy estimate is made through calorimetry: summing the total detector response from the activity in the reconstructed shower. ${ }^{3}$

In neutral current neutrino interactions, the hadronic shower energy is similarly measured through calorimetry. However, the outgoing neutrino (see figure 3.17 ) takes away some fraction of the incoming neutrino's energy, preventing an accurate estimation of the incoming neutrino energy.

The energies of electron neutrinos interacting through the charged current are similarly measured through calorimetry, the response of the detector to electromagnetic showers having been determined from the calibration detector (see section 3.6).

The resolution of muon energy measurement is $6 \%$ using range and $10 \%$ using curvature. The energy resolution for hadronic showers is $(55 \% / \sqrt{\text { energy }})$, and for electromagnetic showers $(23 \% / \sqrt{\text { energy }})$ (see section 3.6$)$.

\footnotetext{
${ }^{3}$ It is possible that improvements could be made by looking at the angle at which the muon exits the shower, to provide information on the $q^{2}$ transferred to the iron nucleus. However, such work is beyond the scope of this thesis.
} 


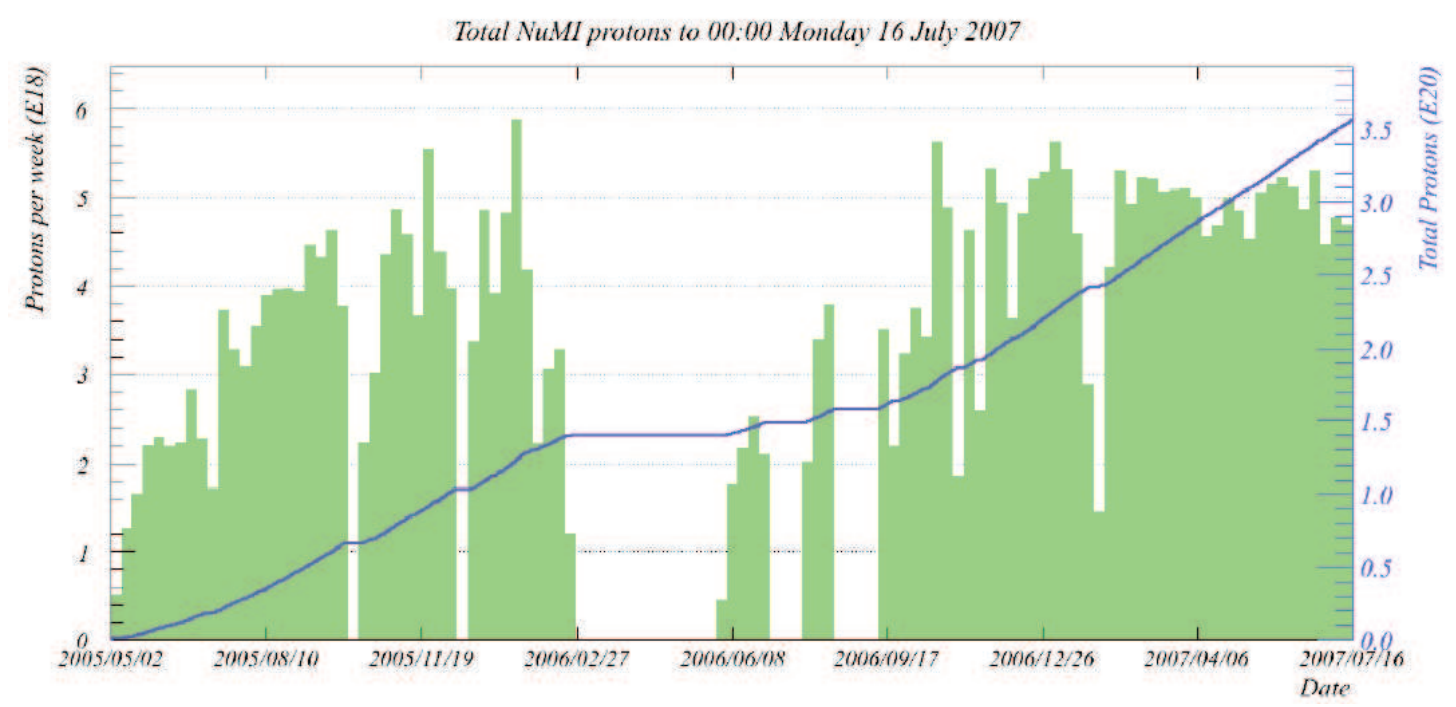

Figure 3.22: The data taken by the MINOS detectors up to June 2007. The green bars show the weekly number of PoT. The blue line shows the cumulative number of PoT.

\subsection{Data from the NuMI Beam}

By July 2007 MINOS had recorded data from $3.6 \times 10^{20}$ PoT, as shown in figure 3.22. The gap in the summer of 2006 corresponds to a shutdown period of the accelerator complex. The two peaks immediately after this shutdown correspond to data taken in the ME and HE configurations (not used for analysis in this thesis). All other data was taken in the LE-10 configuration.

Two data taking periods will be used for analyses in this thesis, totalling $3.21 \times 10^{20}$ PoT (after data quality cuts). The LE-10 running before the 2006 shutdown corresponds to $1.27 \times 10^{20}$ PoT; after the shutdown $1.94 \times 10^{20}$ PoT of LE-10 data are used.

\subsection{Outlook}

The MINOS experiment plans to continue data taking at least into 2010, reaching a total exposure in the region of $8-10 \times 10^{20}$ PoT. With this data, MINOS aims to improve the accuracy of its existing measurements, along with the publication 
of a $\nu_{e}$ appearance measurement. A further possibility is that of reversing the current in the NuMI focusing horns, creating a $\bar{\nu}_{\mu}$ beam (along with a reversal of the magnetic fields in the detectors). This would allow much stronger limits to be placed on $\mathcal{C P} \mathcal{T}$ violation than those set by this thesis. 


\section{Chapter 4}

\section{Drift Calibration}

\subsection{Shower Energy Calibration}

As was discussed in section 3.8.4, the energies of neutrinos interacting in the detectors are reconstructed by adding the measured shower energy to the measured

track energy. Section 3.1.1 explained that a measurement of $\Delta m_{\mathrm{atm}}^{2}$ or $\Delta \bar{m}_{\mathrm{atm}}^{2}$ is performed by looking at the energy dependence of $\nu_{\mu}$ or $\bar{\nu}_{\mu}$ disappearance. Inaccuracies in shower energy measurement therefore produce inaccuracies in the measured oscillation parameters. Shower energy measurement is particularly important in analyses of $\mathrm{NC}$ and $\nu_{e} \mathrm{CC}$ events in which no reconstructed track is expected: the energy of the stereotypical event is measured purely from the shower.

Shower energies are measured through calorimetry. The measured pulse heights of the hits in the shower are summed and scaled directly to a shower energy measurement. This scale factor must be accurate at all times, in every part of each detector, and across the full range of possible shower energies.

The relative scale factor between the two detectors is similarly important. In using the near detector data to predict an unoscillated far detector neutrino spectrum (extrapolation: see chapter 7), an incorrect relative energy calibration causes the far detector spectrum to be predicted with an unphysical energy shift, result- 
ing in erroneous oscillation parameter measurements. In extrapolation methods that do not tune the Monte Carlo shower energy scale ${ }^{1}$, relative energy calibration comes to mean knowing individually the energy scale between near detector data and near detector Monte Carlo, and the scale between far detector data and far detector Monte Carlo.

MINOS aims to know its absolute and relative shower energy scales to better than $5 \%$ and $2 \%$ respectively such that a $\Delta m_{\text {atm }}^{2}$ measurement can be made to better than $10 \%$ before becoming systematically limited. ${ }^{2}$

No controlled source of known-energy particles is available for calibration. Instead, cosmic muons are utilised as they form a plentiful, continuous source of particles with well understood energy deposition processes. Although the cosmic muons are typically more vertical than the horizontal muons arising from beam neutrino events, this is of secondary importance as the main aim is simply to obtain a standard candle with which to track the detector response over time and across the detector; the cosmic muon flux is constant enough to provide this. In situations where the absolute energy deposition per plane is important, the response can be corrected to account for the muon's angle (and therefore path length through each detector plane).

Used alongside cosmic muons are the purpose-built light injection system described in section 3.4 and the calibration detector introduced in section 3.6. These create a calibration chain that is applied to detected pulse heights.

\footnotetext{
${ }^{1}$ Such an extrapolation method was used for the first published MINOS $\nu_{\mu}$ disappearance measurement [158] and is furthermore used in this thesis.

${ }^{2}$ The first published MINOS $\nu_{\mu}$ disappearance measurement assumed uncertainties of $\pm 6 \%$ and $\pm 2 \%$ on the absolute and relative shower energy scales respectively [159]. Work, including that described in this chapter, will allow these uncertainties to be reduced for future analyses.
} 


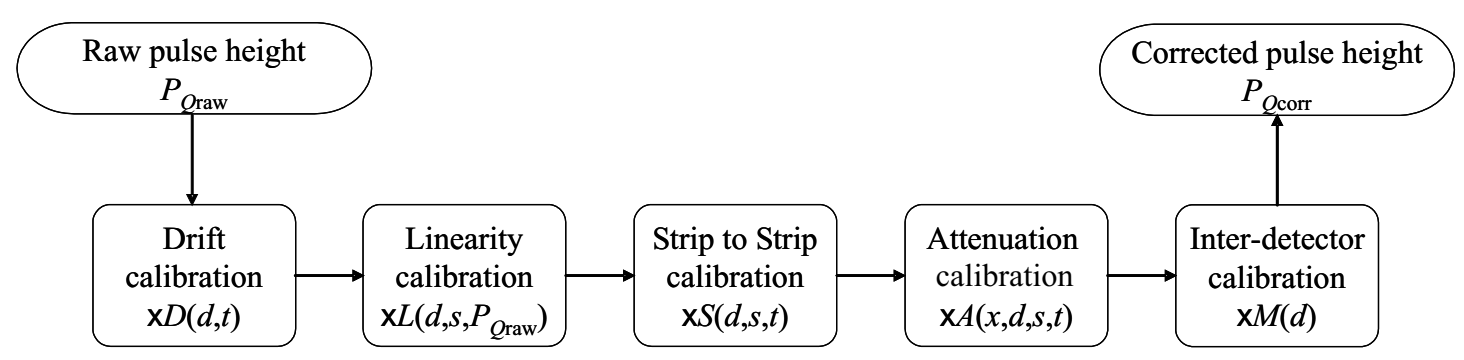

Figure 4.1: The chain of calibrations applied to a raw detected pulse height $P_{Q \text { raw }}$. Each calibration applies the stated multiplicative correction factor to the pulse height. Each correction factor is a function of some subset of $P_{Q \text { raw }}$ and the distance $x$ from the readout end of the relevant scintillator strip $s$, in detector $d$ at time $t$.

\subsection{The Calibration Chain}

The chain of calibrations applied to detected pulse heights is shown in figure 4.1 and dealt with in more detail in [140] and [160].

This chapter goes on to explain in detail the motivation for and procedure of the drift calibration. The calibration looks at the response of each detector to cosmic muons in order to track and correct for changes in the overall detector response with time.

The PMT response depends non-linearly on the amount of input light above signal sizes of $\sim 100$ photoelectrons [161, 162]. At a similar signal size, the far detector readout electronics begin to saturate. The light injection system is used to administer to the PMTs a series of light pulses of varying intensities from of order one to of order hundreds of photoelectrons. Comparing the pulse measured with the detector readout to the known pulse size allows the non-linearity of the readout system to be characterised and a pulse-height dependent correction factor to be calculated on a strip-by-strip basis. At the near detector the size of the light pulse is measured by PIN diodes, known to be linear to within $\sim 1 \%$ up to signal sizes corresponding to 100 photoelectrons at the PMT, and to within $2 \%$ above that. At the far detector the double-ended readout of the strips is exploited. Light is injected at one end of the strip. It is read out at the far end of the strip where 
attenuation means the pulse is within the linear readout range.

Each scintillator strip gives a different response to a similar energy deposit, due to different scintillator, WLS fibre and optical connection efficiencies, readout fibre lengths, PMT gains and electronics responses. The strip to strip calibration uses cosmic muons to correct the response to a hit at the centre of each strip to be uniform across each detector [163]. A correction factor $S$ for each strip $s$ in detector $d$ (near or far) at time $t$ can be calculated by looking at the mean response of each strip to muons which pass through it:

$$
S(s, d, t)=\frac{\text { Mean response of detector } d \text { to stopping muons }}{\text { Mean response of strip } s \text { to stopping muons }} .
$$

Light attenuates as it travels along a WLS fibre. A pulse height must be corrected to account for the distance between the energy deposit itself and the readout end of the strip. On construction of the detector a $662 \mathrm{keV}$ photon source $\left({ }^{137} \mathrm{Cs}\right)$ was used to illuminate closely-spaced points along the length of each scintillator strip. The measured response to these photons is fitted to a double exponential function as a function of distance $x$ from the strip's readout end

$$
R(x, d, s, t)=R_{1}(d, s, t) e^{-x / L_{1}(d, s, t)}+R_{2}(d, s, t) e^{-x / L_{2}(d, s, t)} .
$$

A measured pulse height can now be corrected with the multiplicative attenuation factor $A(x, d, s, t)=(R(x, d, s, t))^{-1}$.

The response of each detector to an energy deposit may still be different. The inter-detector calibration [160] corrects for this. The energy deposition of muons in the detectors follows the Bethe-Bloch formula $[164,165]$ (see figure 4.3 for muon energy loss in copper). Taking a muon that stops in the detector, the energy deposited a known distance from the end of the track can be calculated from this model. From a sample of stopping muons, the average response of each detector to a muon of known energy can be measured and pulse heights are corrected to 
make this uniform between the detectors.

It remains to relate detected pulse heights to the interacting particle energies. As described in section 3.6 known-energy beams of hadrons and leptons (including muons) were aimed at the calibration detector. These data allowed the responses to the various types of particle to be related to each other. The stopping muon inter-detector calibration was also performed at the calibration detector [160] to allow these responses to be related to the near and far detectors.

A shower-related calibration falling outside the calibration chain is that of PMT gains (including also the more stable electronics response). Knowing the gains is important to identify cross talk (which is expected at the 1 photoelectron level), and to enable correct modelling of the effect of photon statistics on shower topology in the Monte Carlo. The gains change due to hardware swaps and the aging of PMTs. The light injection is again used: every strip end is regularly (on the order of seconds) illuminated with a light pulse. Comparing the readout signal with the input light after linearity correction gives the required gain measurement.

\subsection{The Need for a Drift Calibration}

The responses of the scintillator, WLS fibres, PMTs and electronics are not constant over time. Test-stand measurements were conducted before detector construction to investigate such variations [140].

Scintillator light output was measured to decrease due to aging by $1.2 \%$ per year at $20^{\circ} \mathrm{C}$, with faster aging at higher temperatures. An additional recoverable decrease in scintillator light output was measured at $0.3 \%$ per $1^{\circ} \mathrm{C}$ increase in temperature (the temperature in the detector halls has varied by up to $10^{\circ} \mathrm{F}$ during data-taking).

The attenuation length of the WLS fibres was also shown to decrease with age. This results in the light output at the end of a typical $8 \mathrm{~m}$ fibre decreasing by 
$1.2 \%$ per year at $20^{\circ} \mathrm{C}$, again with faster aging at higher temperatures.

The far detector M16 PMTs were shown to have a gain decrease of $0.2 \%$ per $1^{\circ} \mathrm{C}[166]$, and the near detector M64 PMTs a gain decrease of $<0.6 \%$ per $1^{\circ} \mathrm{C}$ [167]. Furthermore, at the beginning of their lives PMT gains increase with time; but after a while (of order a year at the operating current of MINOS) this gain increase stops, and eventually the gains begin to decrease with time [168].

The electronics, although intrinsically comparatively stable, are affected by hardware swaps. At the far detector very few hardware swaps are made (of order two or three per year), each affecting only a small portion of the detector. At the near detector a much larger number of swaps are made (of order one per week) so the effect is larger; however the electronics are internally calibrated when swapped in order to minimise the effect on detector response.

The inter-detector calibration is performed once only for each detector and requires a large dataset, covering a period of several months (chosen to be those months over which the beam data was taken). A drift in detector response over this period means the calculated energy scale is not truly applicable to all the data: a constant drift in the detector response during the data taking period means that no energy scale factor is correct at all times. This will worsen the detectors' energy resolutions.

A calibration is therefore necessary to track, and correct for, the time-dependent drift of the detector response.

\subsection{Drift Calibration Technique}

\subsubsection{Cosmic Muons}

The response of the detectors is tracked by looking at the median response of a detector plane to cosmic muons. The response used is the plane-summed pulse 
height: the sum of all pulse heights in each plane associated to the track by the reconstruction software.

The spectra of cosmic ray muons are different between the two detectors, with differing rates, energy spectra and angular distributions. For example, throughgoing cosmic muons have, at the Near and Far Detectors respectively, approximate rates of $\sim 10 \mathrm{~Hz}$ and $\sim 0.5 \mathrm{~Hz}$ and approximate mean energies of $\sim 75 \mathrm{GeV}$ and $\sim 200 \mathrm{GeV}$. Factors contributing to this include the different depths of the detectors (different rock overburdens), and different latitudes resulting in variation in the Earth's magnetic field. However, these differences are non-time-dependent so do not affect the calibration as no absolute energies are calculated: the calibration aims merely to relate response at one time in one detector to that at a different time in the same detector.

Cosmic muons are considered to be any reconstructed track occurring outside of a beam spill. This assumption is well justified (particularly as the track, as detailed in section 4.4.5, is required to be greater than eight detector planes in length) as little other than a muon can cause a long track in an underground detector. Furthermore, as no absolute absolute energies are calibrated, if any backgrounds were present they would only become important if the resultant energy deposited in the detector changed as a function of time. The first and last planes of each muon track are not used. This removes the high energy loss in the last plane of a stopping muon. It furthermore eliminates planes in which non-contained muons enter and leave the detector and may only clip the plane, giving an anomalously low energy deposit.

The only conceivable physical process creating a non-muon track-like event in the detector is the interaction of a cosmic electron neutrino (the rate of which will not change as a function of time). A prediction of the electron neutrino rates in the Soudan2 detector (in the same cavern as the MINOS far detector now occupies) was made in [169]: 74.57 events per kiloton per year. Scaling this 
to the MINOS far detector implies a rate of the order one interaction per day or $\sim 10^{-5} \mathrm{~Hz}$ : negligible compared to the cosmic muon rate. At the near detector this rate will be even lower (the detector mass is less whilst the lower rock overburden has negligible effect on neutrino fluxes) whereas the cosmic muon rate increases dramatically. A further concern would be that the electron neutrino produces an electromagnetic shower which deposits much more energy per plane than a muon track. However section 4.4.7 details the choice of the daily median plane-summed pulse height as the measure of detector response. The use of the median largely eliminates sensitivity to such spasmodic large energy depositions.

A change over time in the overall energy spectrum of the muons could invalidate their use as a standard candle. The only potential mechanism for this would be a change in the energy spectrum of the primaries (predominantly protons) at the top of the atmosphere. The energy at the earth's surface of muons passing through the MINOS detectors are above $\sim 1 \mathrm{TeV}$ at the near detector and above $\sim 10 \mathrm{TeV}$ at the far detector. Variations in the solar magnetic field cause the primary energy spectrum to vary over the 11 year solar cycle; but this only affects muons below $\sim 20 \mathrm{GeV}$ surface energy [169]. A further possibility is a change in the energy spectrum of primaries trapped in the galactic magnetic field. Simple models [170] suggest that protons up to $\sim 10^{5} \mathrm{TeV}$ can have a non-negligible confinement time in the galactic field: this covers the region relevant to MINOS. An injection of protons by some galactic event (such as a supernova) could affect the overall energy spectrum, but is neglected as an unlikely scenario. Beyond this, only a correlated change in the energies of primaries emitted by astronomical bodies, correlated over time to their distance from the earth, could cause a systematic effect on the calibration: a highly improbable situation.

The energy spectrum of cosmic rays has been precisely measured over the last few decades: the spectrum of primaries is consistently found to go as (energy) ${ }^{-2.7}$ (see figure 24.1 of [171] for a compilation of data from the last 20 years). This 
demonstrates experimentally the constancy of the cosmic ray spectrum over time, and validates the use of cosmic muons as a standard candle in MINOS.

Changes in the rate of cosmic muons over time do not affect the calibration as the median response is not affected. Seasonal changes in the angular distribution of muons would be a systematic effect on the calibration as a muon at a steeper angle deposits more energy per plane. The experimental results which demonstrated in the previous paragraph the invariance of the cosmic primary energy spectrum over time also demonstrate its invariance geographically (as the experiments were performed at different locations). The only mechanism which could change the muon angular distribution underground at the MINOS detectors would be a change in the rock overburden, and this has been constant over the duration of the experiment. (The magnetic field of the Earth is not a concern as it is too weak to affect the high $(\mathrm{TeV})$ energy primaries which correspond to the underground muon flux.) Furthermore, section 4.4.2 details a path length correction applied to the muon tracks which will remove any residual angular distribution effects.

Few detector effects can mimic a long track as the majority of noise comes from the WLS fibres in the form of single, small pulses of light. The only possibility is that a pulse of light injection (see sections 3.4 and 4.2) is reconstructed as data, the reconstruction software being able to form a track out of such hits. This was believed to be guarded against as all light injection pulses should be tagged as such. However studies following on from those of this chapter [172] have shown that, in the early days of the far detector lifetime, this tagging occasionally failed and a small number of light injection pulses have had a small effect on the calibration. However, this was long before any NuMI beam data was taken. 


\subsubsection{Path Length Correction}

The further the direction of a muon from the perpendicular to a detector plane, the more material the muon passes through per plane (greater path length), so the more energy it deposits per plane. Any change over time of the angular spread of cosmic muons is negligible on the level of the temperature and aging-related changes in detector response, so a path length correction is not critical. However, the RMS spread of the plane-summed pulse heights will be reduced if such a path length correction is applied, reducing the uncertainty on the calibration constants.

Taking the total path length of the muon through the detector, $d$, and total distance travelled by the muon along the perpendicular to the planes, $z$, the energy deposited in the $i$ th plane, $E_{i}$, is corrected to give the path length corrected planesummed pulse height, $E_{i}^{\prime}$ :

$$
E_{i}^{\prime}=\frac{z}{d} E_{i}=\frac{E_{i}}{R}
$$

where $R=d / z$ is defined as the path length correction factor.

As every hit on a track is corrected by the same factor, this is only an exact correction under one of two approximations: that the track is straight or that the energy deposited is constant along the path of the muon.

As the detectors are magnetised, the charged muon tracks bend. The steepest parts of the track are therefore under-corrected and the shallower parts overcorrected. However, the typical cosmic muon energies are high (of order $100 \mathrm{GeV}$ ) so the tracks are typically close to being straight (a typical cosmic muon in the far detector is shown in figure 4.2). For example, a $100 \mathrm{GeV}$ muon travelling perpendicular to the magnetic field will have a large radius of curvature of $\sim 250 \mathrm{~m}$; cosmic muons, being primarily vertical, are not typically perpendicular to the field so curve even less than this estimate.

The rate of energy loss of muons passing through copper is shown in figure 4.3. The behaviour in iron is not significantly different. Muons travelling through the 


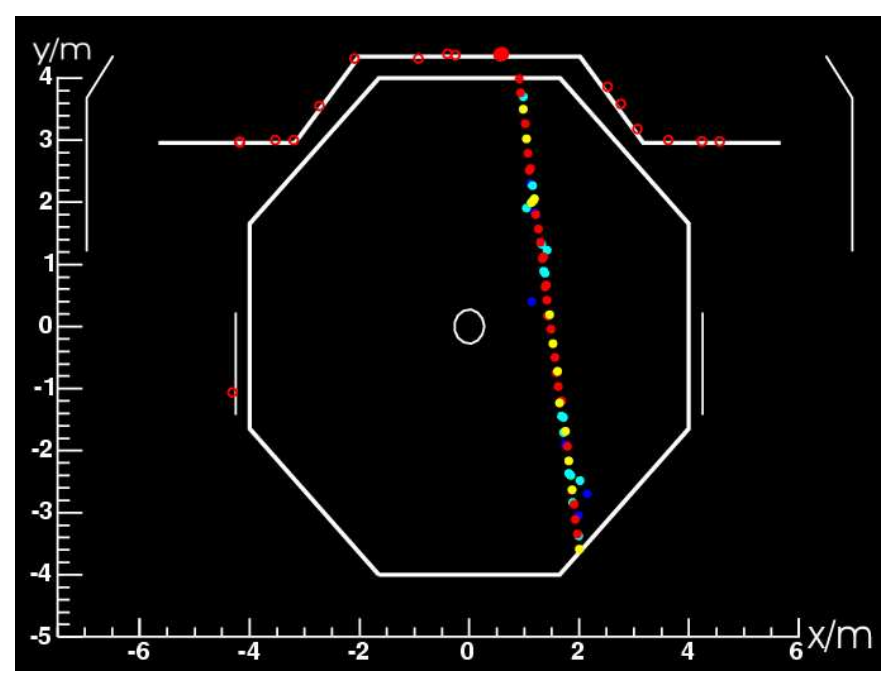

Figure 4.2: An event display showing a typical cosmic muon (from data) in the far detector. The display shows (online) reconstructed detector hits in the $x y$ co-ordinate view (looking downstream along the beam axis). The large white octagon represents the detector outline. The central white circle represents the coil hole. The hits outside the main octagonal detector planes (along the white lines) represent hits in the veto shield (a layer of scintillator strips above the detector, used in cosmic ray physics analyses).

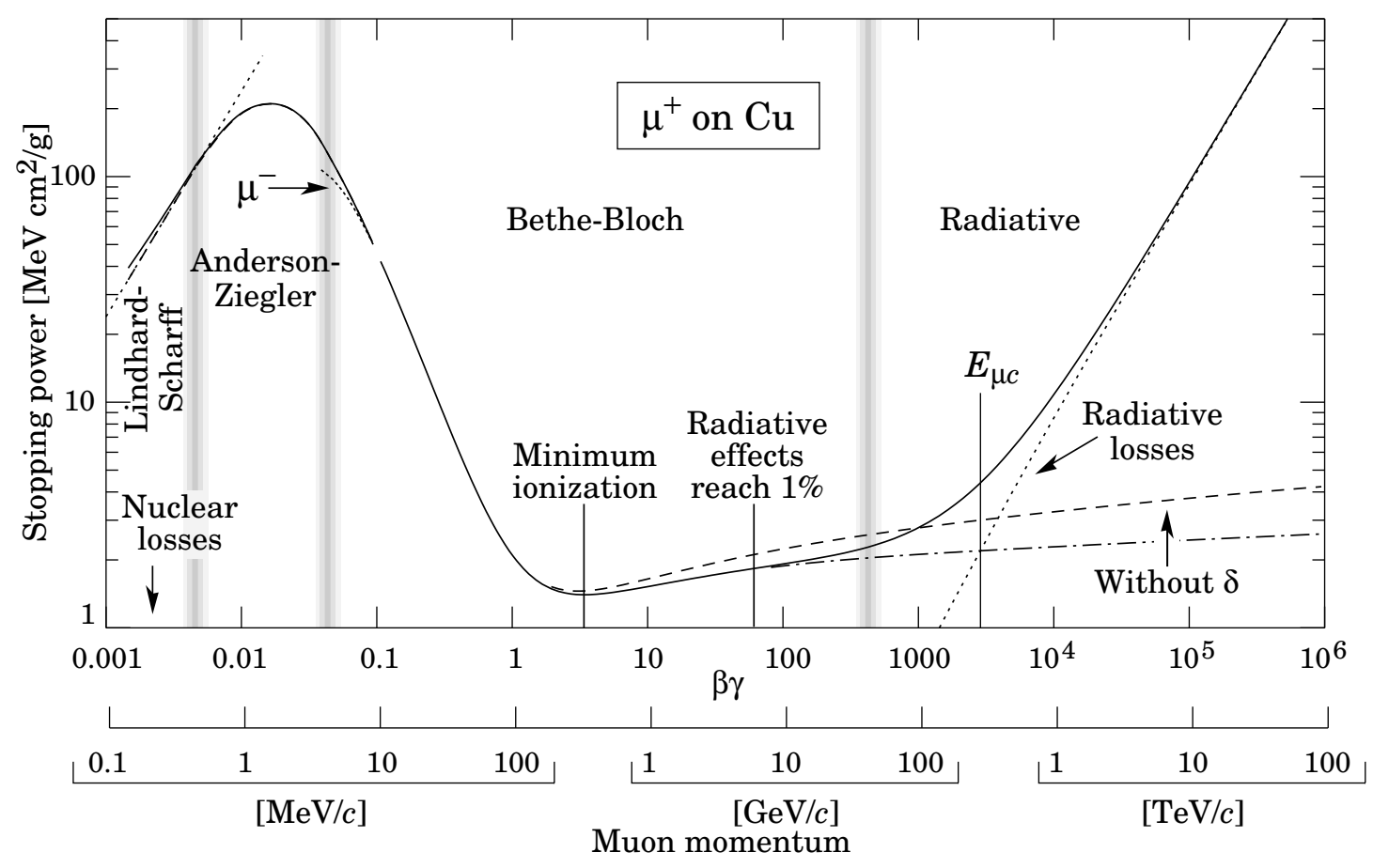

Figure 4.3: The stopping power of muons in copper as a function of muon momentum. The region important in MINOS (stopping power in iron is not significantly different from copper) is the flattest part of the Bethe-Bloch region between $1 \mathrm{GeV}$ and a few hundred GeV. Figure taken from [58]. 


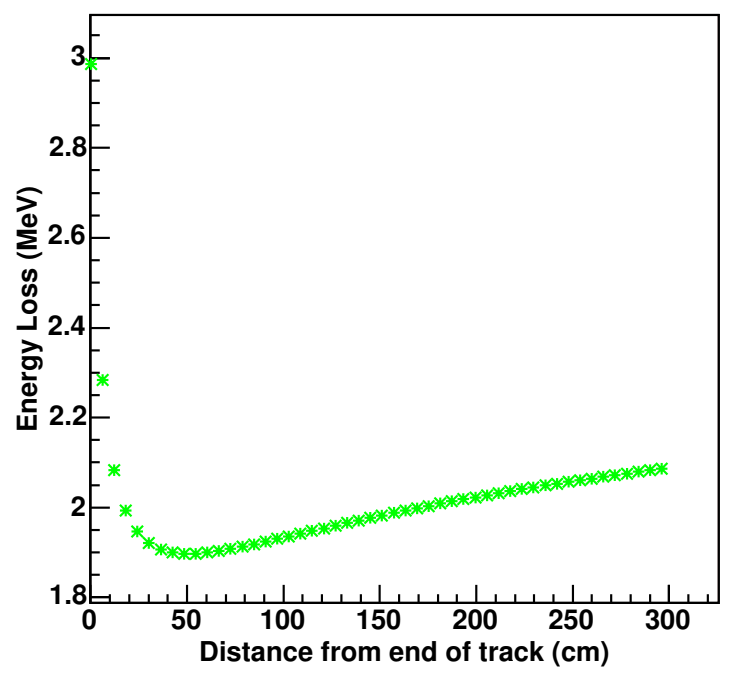

Figure 4.4: The energy loss per plane of muons in scintillator. Plot taken from [160].

detectors deposit energy predominantly through ionization. Radiation losses in iron are only consistently of a comparable level for muons of more than a few hundred $\mathrm{GeV}$. From the figure, the energy loss for a muon is in its flattest region from $1 \mathrm{GeV}$ up to a few hundred GeV. At the low-energy end, the muon energy loss in the scintillator is shown as a function of distance from the end of a stopping muon track in figure 4.4. The energy loss does not deviate far out of the flat region even down to the final $10 \mathrm{~cm}(\sim 2$ planes $)$ of the track.

As the path length correction is not critical to the calibration, the level of obeyance to these approximations is considered good enough for the method to be implemented.

The pulse height deposited by every plane hit by a sample of cosmic muons is plotted in figure 4.5 as a function of $R$ before and after path length correction. The path length correction successfully flattens the response of the planes to muons as a function of path length of the muon through the plane. 

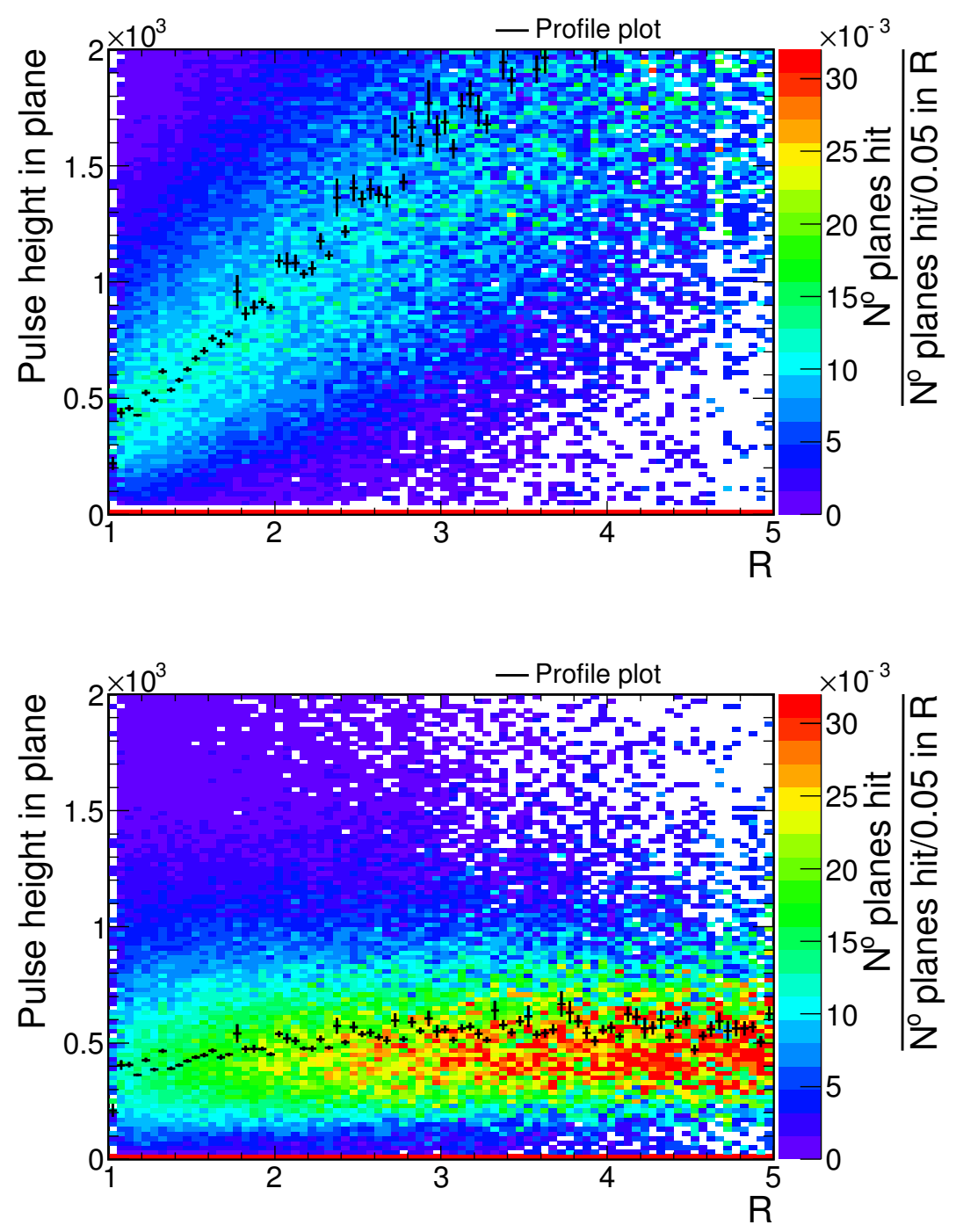

Figure 4.5: The pulse height deposited in every plane hit by a sample of cosmic muons in the near detector, plotted against the path length correction factor $R$ (as defined in equation 4.1). Shown before (top) and after (bottom) performing the path length correction. Every entry is normalised such that integrating over each column of bins yields unity. The orange line shows the corresponding profile plot of the mean pulse height in the plane as a function of $R$. 


\subsubsection{Hit Positions}

To enable fiducial cuts to be performed, the pulse-height weighted mean position $\left(\langle x\rangle_{\mathrm{ph}},\langle y\rangle_{\mathrm{ph}}\right)$ of all tracked hits in each plane is calculated. If the $x$ co-ordinate of the $i$ th hit in the plane is $x_{i}$, which deposits a pulse height $P_{Q i}$, then

$$
\langle x\rangle_{\mathrm{ph}}=\frac{\sum_{i} x_{i} P_{Q i}}{\sum_{i} P_{Q i}}
$$

and similarly for $y$.

\subsubsection{Zero-Plane Reconstruction}

Any planes between the first and last planes of the track that record no pulse height are registered as zero pulse height planes. This is necessary so that the calibration is correctly sensitive to Poisson fluctuations of hits at the 1 photoelectron level.

The zero-planes are given an effective pulse-height weighted mean position by extrapolation between the previous and next non-zero planes to enable fiducial cuts to be performed on these hits. If the previous non-zero plane has mean position $\left\langle x_{\mathrm{p}}\right\rangle_{\mathrm{ph}}$ and was $p$ planes previous, whilst the next has $\left\langle x_{\mathrm{n}}\right\rangle_{\mathrm{ph}}$ and is $n$ planes further on, the current zero-plane is given a position

$$
\left\langle x_{0}\right\rangle_{\mathrm{ph}}=\frac{n\left\langle x_{\mathrm{p}}\right\rangle_{\mathrm{ph}}+p\left\langle x_{\mathrm{n}}\right\rangle_{\mathrm{ph}}}{p+n}
$$

and similarly for $y$ (the $u$ and $v$ co-ordinates of each plane could also have been used for this purpose; in a reconstructed track the two are equivalent).

The reconstructed positions of zero-planes are shown for each detector in figure 4.6, along with photographs showing the scintillator plane structure. The reconstructed zero-plane positions lie in the coil hole and along the gaps between scintillator modules. Some lie outside the detector, due to badly reconstructed 

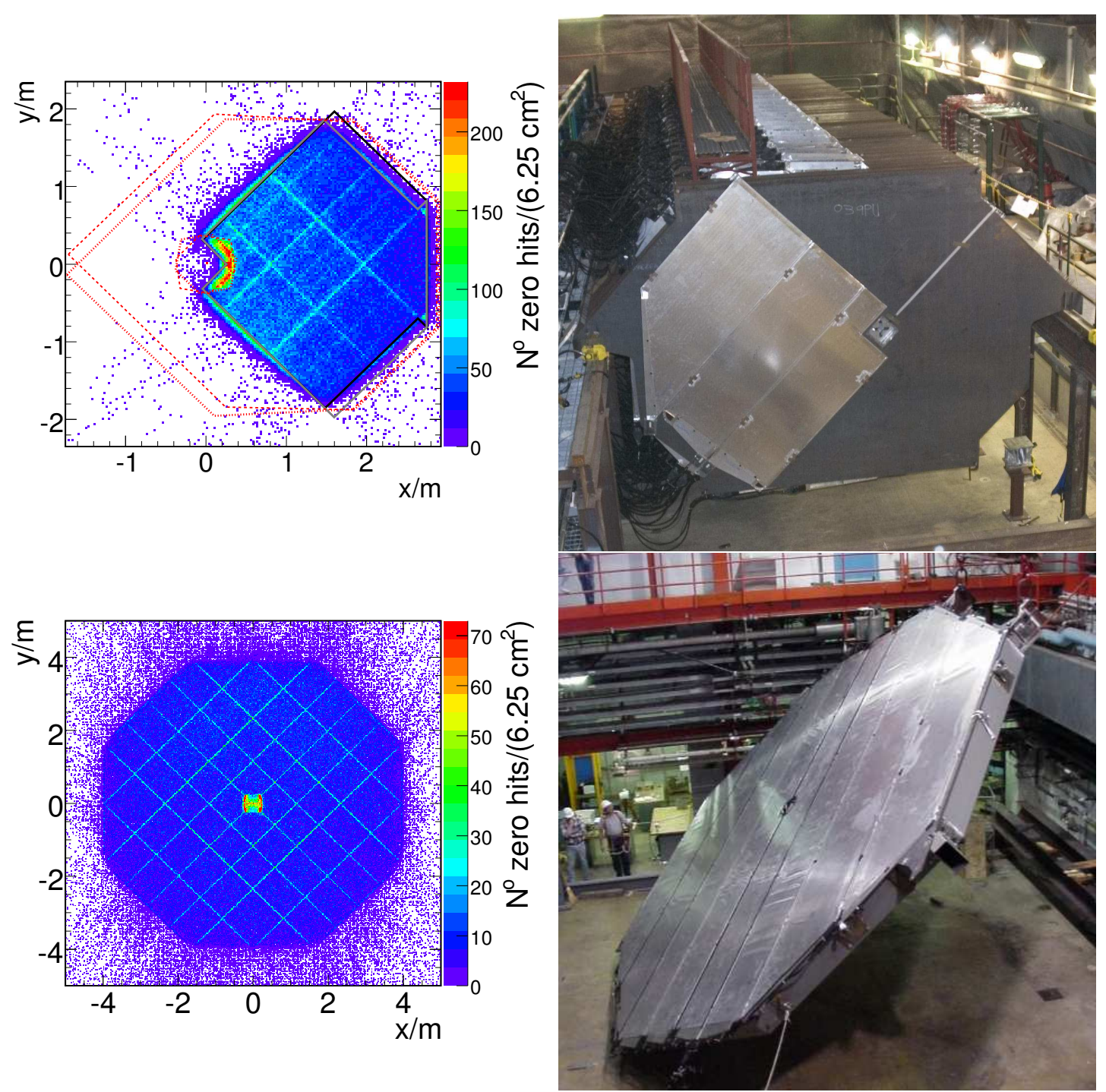

Figure 4.6: Left: the reconstructed pulse-height weighted mean positions of zeroplanes in the near (top) and far (bottom) detectors. Superimposed on the near detector graph is the detector plane outline. Right: photographs of the corresponding detectors whilst under construction, showing the pattern of scintillator modules. 


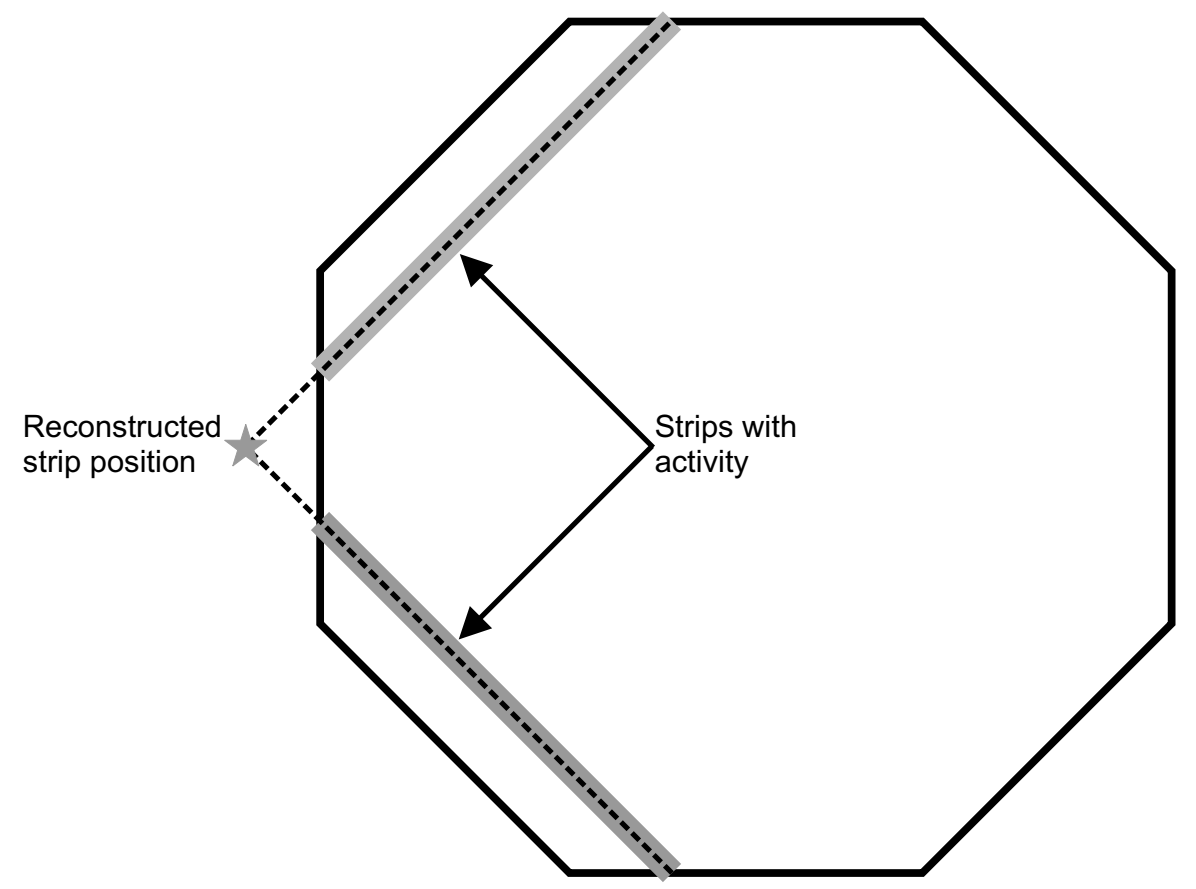

Figure 4.7: An illustration of how a reconstruction failure can result in detector activity being assigned a position outside the physical detector.

tracks, as demonstrated in figure 4.7. Activity on two strips which do not meet within the physical detector can be associated together; the reconstruction assigns a three-dimensional position to this activity at the point where the two strips would cross if extended outside the detector. This is a pathology which should be rectified in future versions of the software. The low level at which this happens is not considered a problem for this calibration, and these planes are removed by the fiducial cuts detailed below.

\subsubsection{Track Selection}

The detector readout period containing the reconstructed muon must contain only one reconstructed track and must not have been triggered by a spill trigger. There must be at least nine planes (inclusively) between the first and last planes hit (the track need not register a hit in the intermediate planes). This criterion makes the reconstructed track less likely to be a misidentified shower (e.g. from a cosmic $\nu_{e}$ ). 
The value of nine planes was not carefully optimised, merely obtained by looking at events in the detector which looked like good muons. The track's path length correction factor $R$ must be less than 3.0. This cuts out very steep tracks (steeper than $\sim 70^{\circ}$ to the horizontal).

\subsubsection{Plane Selection}

As was explained in section 4.4.1, the first and last planes hit in each track are discounted to remove the high energy loss in the last plane of a stopping muon and planes which non-contained muons may only clip. To further remove low energy deposits from tracks only clipping planes, the plane-summed pulse height in the track is stored if the pulse-height weighted mean position (section 4.4.3) of the hits is considered to be within a fiducial region on the plane.

The distance of $\left(\langle x\rangle_{\mathrm{ph}},\langle y\rangle_{\mathrm{ph}}\right)$ from the centre of the coil hole is

$$
\langle r\rangle_{\mathrm{ph}}=\sqrt{\langle x\rangle_{\mathrm{ph}}^{2}+\langle y\rangle_{\mathrm{ph}}^{2}}
$$

In both detectors, this is required to be $>30 \mathrm{~cm}$ from the coil hole centre. In the Far Detector it is additionally required to be $<3.5 \mathrm{~m}$ from the coil hole centre. In the Near Detector, the additional requirement is to be within a $1 \mathrm{~m}$ radius circle centred $1 \mathrm{~m}$ away from the coil hole in the the positive $x$ direction (see figure 4.6 for the relationship of detector outlines to co-ordinate systems). The same fiducial requirements are applied to the reconstructed zero-planes.

\subsubsection{Tracking Detector Drift}

The distribution of selected plane-summed pulse heights is shown in figure 4.8 (for 24 hours of data) for each detector. This distribution is tracked over time to track the detector response drift. Three attributes of the distribution were investigated: the mean, median and a Gaussian fit to the peak (the fits being 

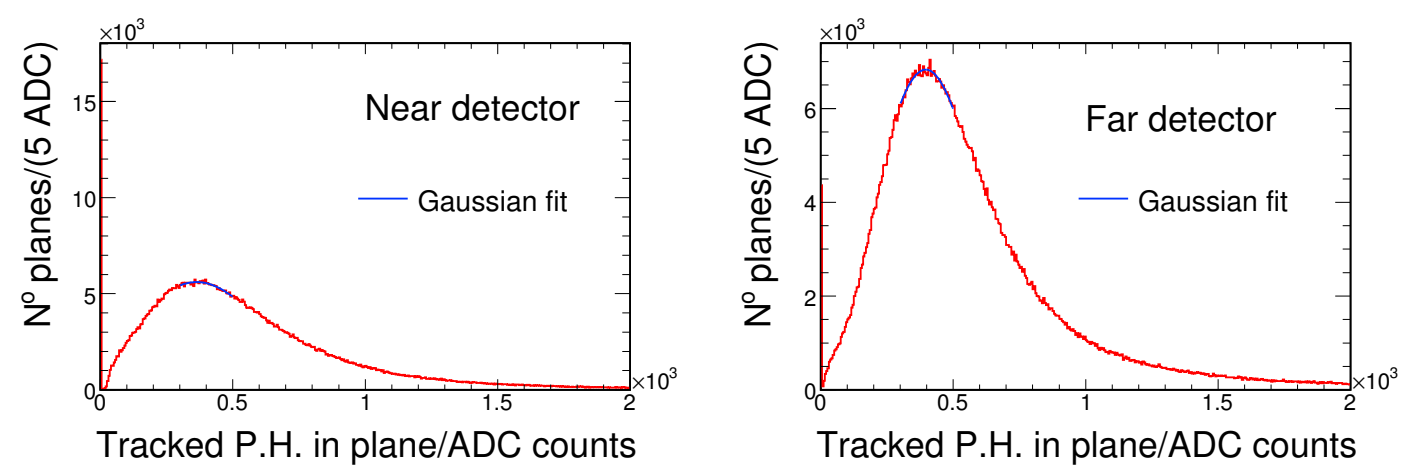

Figure 4.8: The distribution of selected plane-summed pulse heights over a 24hour period in the near (left) and far (right) detectors. The Gaussian peak fit considered for use in tracking the detector drift is shown.

shown in figure 4.8).

The mean, median and peak fits of 24 hour periods of data are tracked over a three month period in figure 4.9 (near detector) and 4.10 (far detector) with a graph of the corresponding detector hall temperature. The median shows less apparently random jitter and more detailed response to temperature changes in both detectors. The median was therefore chosen for use in tracking the detector drift.

The error bars on the mean detector response are the error on the mean:

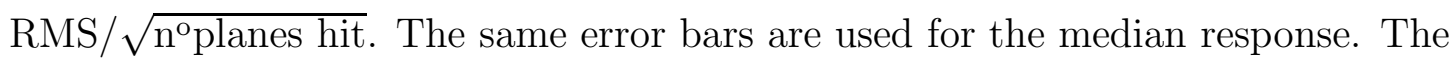
Gaussian fit to the peak is performed using ROOT's internal fitting function [173]. The error bars are the errors reported by the fit on the position of the peak.

\subsubsection{Granularity in Time}

Figures 4.11 and 4.12 show, respectively, the near and far detector response over a two-month time period, with the measured detector hall temperature superimposed. Each figure shows the response calculated as the median of 3-, 8- and 24-hour time periods separately, with hall temperatures averaged (mean) over the same time periods.

The main features occur over periods longer than 24 hours. The data for 

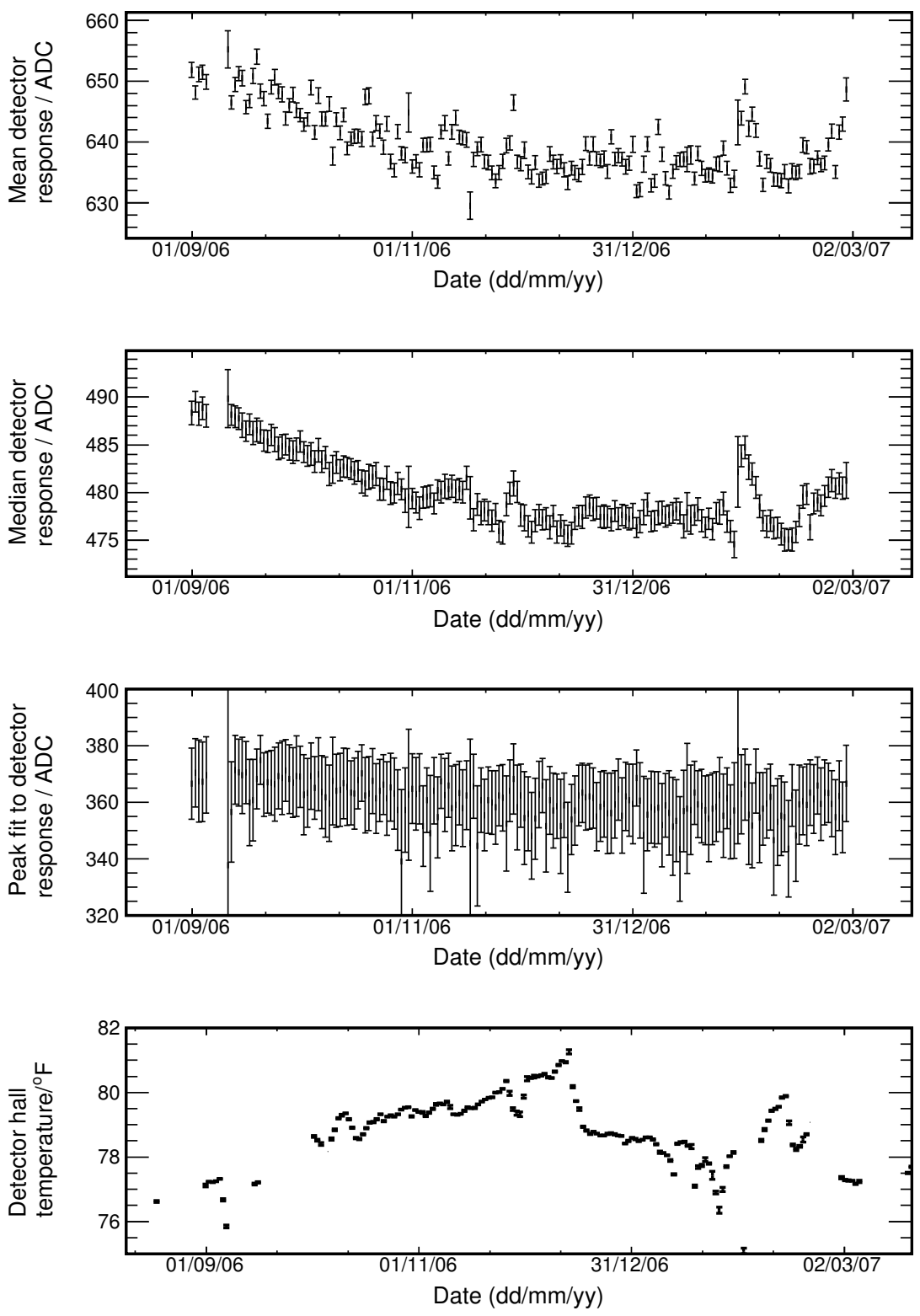

Figure 4.9: From the top, the mean, median, and a Gaussian fit to the peak of the near detector response each day over a six-month period. The bottom graph shows the near detector hall temperature (each point averaged over a day; readings taken every hour) for the same period. The error bars on the mean and median plots are the error on the mean for that day (i.e. the same in both cases):

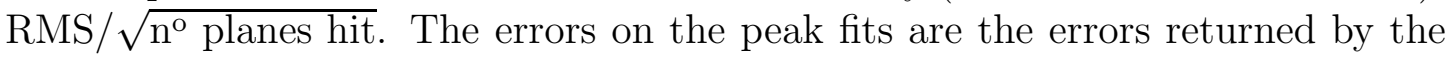
ROOT fitting function [173] on the mean of the Gaussian. The error bars on the temperatures are the error on the mean for that day. 

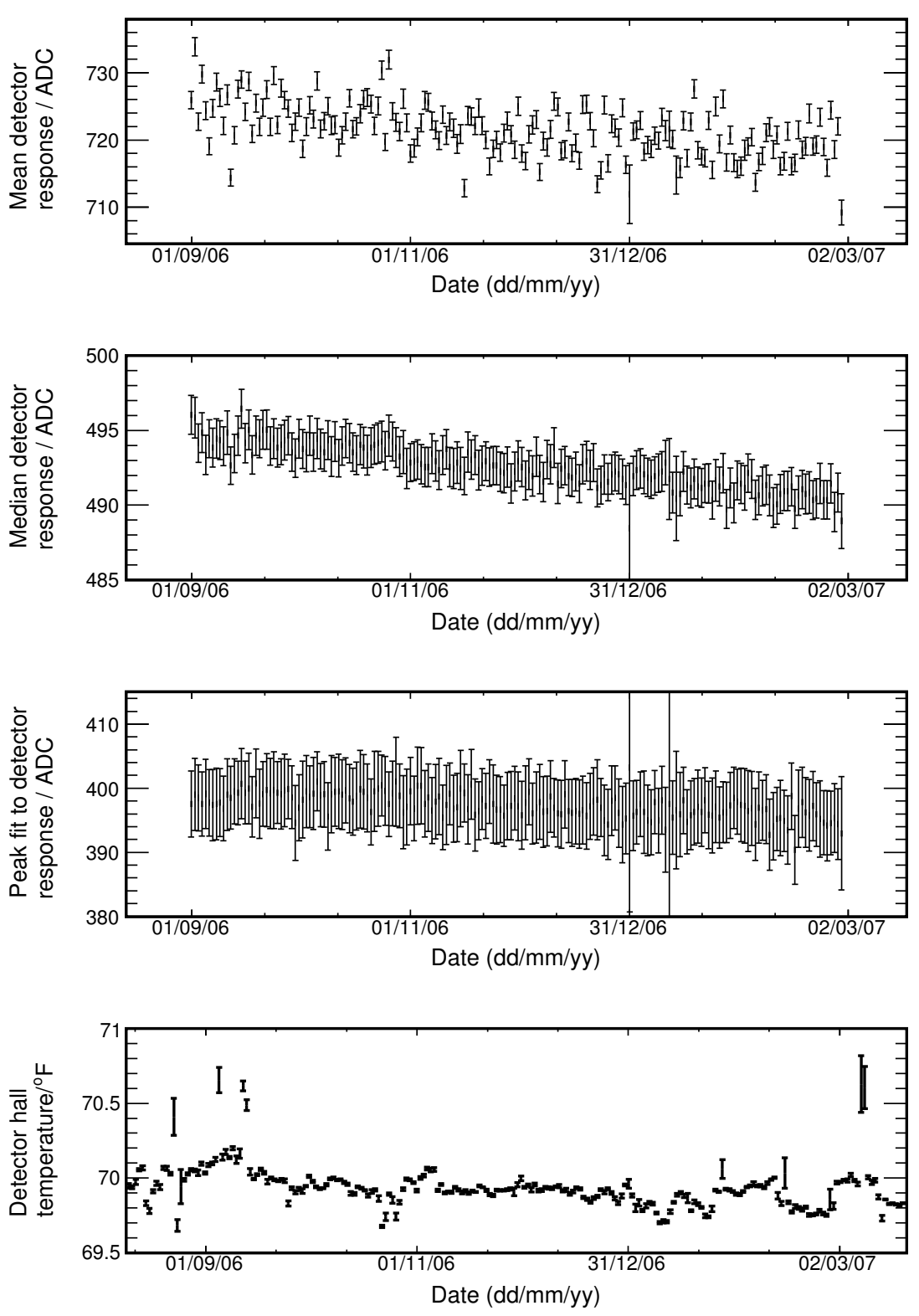

Figure 4.10: The set of graphs equivalent to figure 4.9, here for the far detector. 

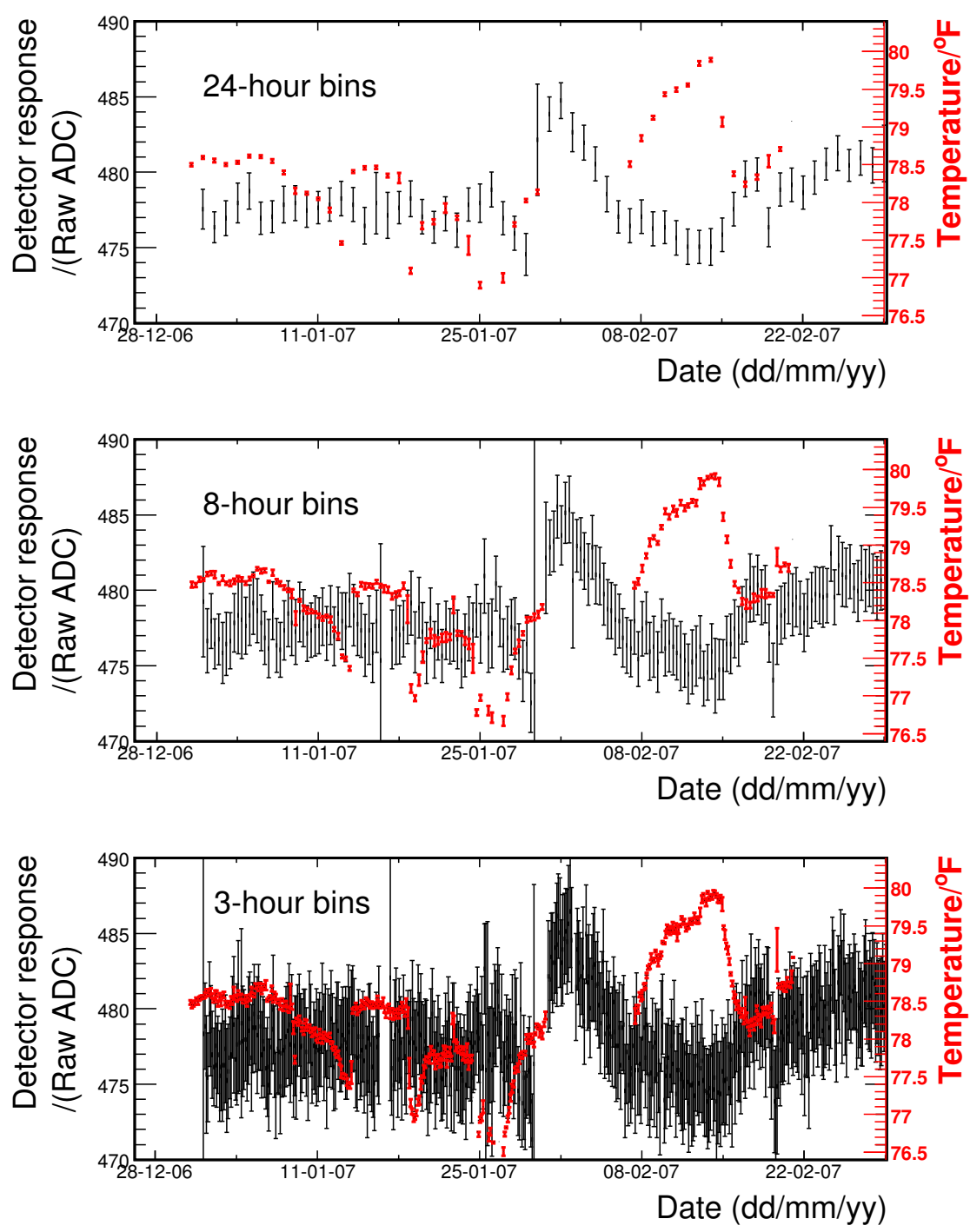

Figure 4.11: The detector response (black) of the near detector over a two-month period, calculated as the medians of 24- (top), 8- (middle) and 3-hour (lower) periods of muon response data. The red graphs show the near detector hall temperatures averaged (mean) over the same time periods in each case. 

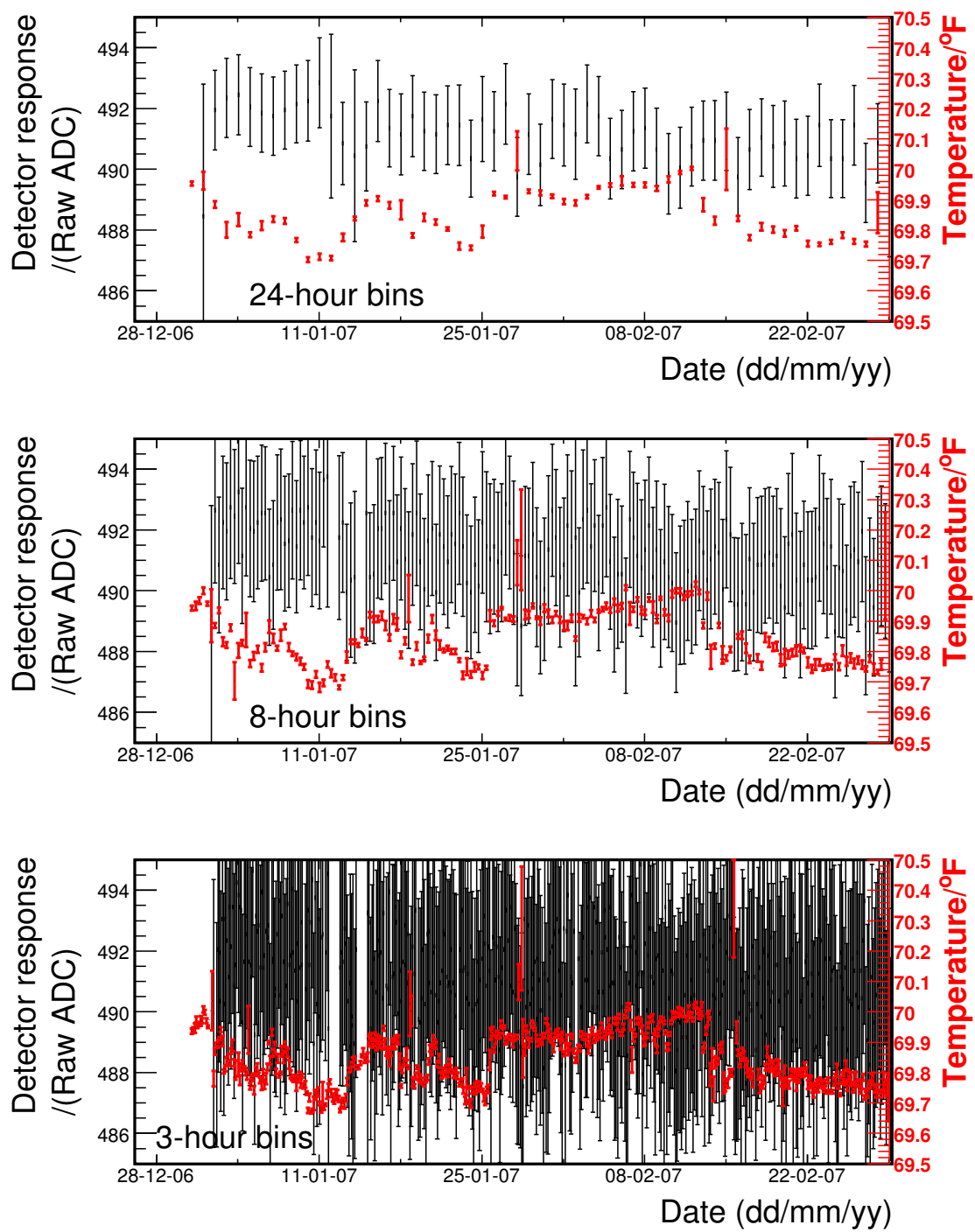

Figure 4.12: The set of graphs equivalent to figure 4.11, here for the far detector. 


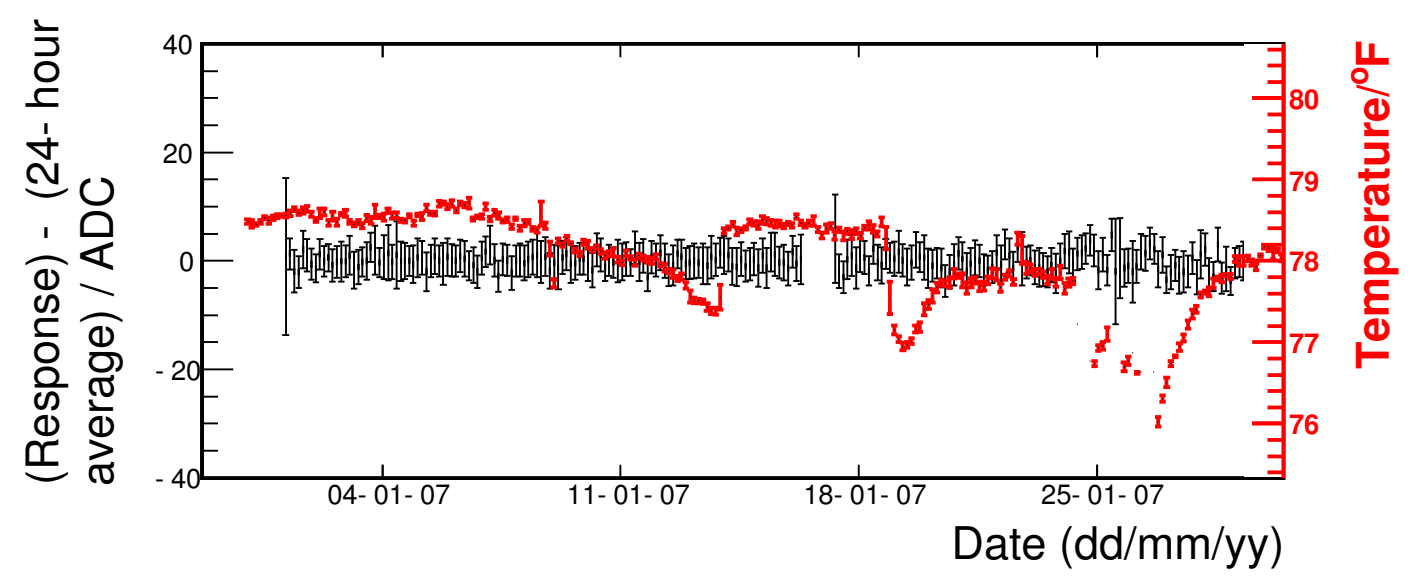

Figure 4.13: Black: the median near detector response for 3-hour time periods, from which has been subtracted the corresponding 24-hour median response. Red: the mean near detector hall temperature in 3-hour periods.

3- and 8-hour periods show no features not visible in that for 24-hour periods, as demonstrated in figure 4.13 in which the 24-hour median response has been subtracted from the 3-hour periods for a period of near detector running. The data for 3-hour periods furthermore shows error bars large enough to wash out some features. 24-hour time periods were chosen as the optimal granularity over which to perform this calibration.

\subsubsection{Version Shear}

Drift constants for periods of detector operation are calculated immediately following that period. Thus not all constants were calculated at the same time. During the period of detector operations to date the required cosmic muon data has been reconstructed with four different versions of software, labelled R1_18, R1_18_2, R1_18_4 and Cedar (table 4.1 shows the dates for which each reconstruction version was used).

Changes in the muon tracking software can cause the reconstructed planesummed pulse height of muons to change. The drift calibration must not correct for these changes as any physics analysis will be performed using data reconstructed 


\begin{tabular}{|l|l|l|}
\hline \multirow{2}{*}{$\begin{array}{c}\text { Reconstruction } \\
\text { software version }\end{array}$} & \multicolumn{2}{|c|}{ Dates calibrated } \\
\cline { 2 - 3 } & \multicolumn{1}{|c|}{ Near detector } & \multicolumn{1}{|c|}{ Far detector } \\
\hline \hline R1_18 & $1 / 3 / 05-30 / 4 / 05$ & $17 / 7 / 03-30 / 4 / 05$ \\
R1_18_2 & $1 / 5 / 05-26 / 2 / 06$ & $1 / 5 / 05-26 / 4 / 06$ \\
R1_18_4 & $30 / 5 / 06-30 / 11 / 06$ & $27 / 4 / 06-1 / 09 / 06$ \\
Cedar & $1 / 12 / 06$ onwards & $2 / 09 / 06$ onwards \\
\hline
\end{tabular}

Table 4.1: The periods of data calibrated using each different reconstruction software version.

with a single software version.

Each time the reconstruction software changed, the detector response for each day in a single two-month period, reconstructed with both software versions, was calculated. The mean of these detector responses was calculated over the full twomonth period, reconstructed with both software versions. and the ratio of these used as a multiplicative factor to correct the responses relative to each other.

For example, to correct the detector response $D_{\alpha}$ calculated with software version $\alpha$ to that $\left(D_{\beta}\right)$ calculated with a version $\beta$ to give a corrected response $D_{\alpha}^{\prime}$

$$
D_{\alpha}^{\prime}=\frac{\left\langle D_{\beta}\right\rangle_{2 \text { months }}}{\left\langle D_{\alpha}\right\rangle_{2 \text { months }}} D_{\alpha}
$$

where the averaging is over two common months of data.

The calculated correction factors are shown in table 4.2. All software versions are corrected relative to R1_18_2.

A systematic error on these correction factors was calculated by recalculating the daily detector response using the mean (instead of the median) plane-summed pulse height. The ratio of the mean responses over the two months was then recalculated. The difference between this and the ratio calculated using the medians was taken as the systematic error on the ratio (also shown in table 4.2).

No data was processed with both R1_18_2 and R1_18_4. To calculate the R1_18_4 correction factor, a factor was calculated to correct R1_18_4 to Cedar; this was multiplied by the Cedar to R1_18_2 factor. As before, this was performed 


\begin{tabular}{|l|l|l|}
\hline Reconstruction & \multicolumn{2}{|c|}{ Correction factor } \\
\cline { 2 - 3 } software version & \multicolumn{1}{|c|}{ Near detector } & \multicolumn{1}{c|}{ Far detector } \\
\hline \hline R1_18 & $1.0010 \pm 0.0068$ & $0.9999 \pm 0.0007$ \\
R1_18_2 & 1 & 1 \\
R1_18_4 & $1.0000 \pm 0.0021$ & $0.9989 \pm 0.0010$ \\
Cedar & $1.0007 \pm 0.0288$ & $0.9669 \pm 0.0437$ \\
\hline
\end{tabular}

Table 4.2: The factors used to correct a detector response calculated using a particular reconstruction software version to a detector response equivalent to that calculated using the R1_18_2 version. Errors are systematic errors.

for the median and mean detector responses and the difference between the results taken as the error.

\subsection{Results of the Drift Calibration}

\subsubsection{Detector Response}

Figures 4.14 and 4.15 show the median response of the near and far detectors respectively over the full period of detector operations to date. Also shown are the respective detector hall temperatures. An inverse correlation between temperature and detector response is clearly visible.

The far detector additionally shows a steady drop in detector response of approximately $2 \%$ per year. The step upwards in the far detector response during March 2006 is due to a retuning of the voltage across the PMTs. The far detector hall temperature becomes dramatically smoother at the end of 2004 when a decision was made to leave the lights on in the detector hall overnight and at weekends, to achieve a more constant temperature.

The near detector shows a constant response over the first year of livetime, consistent with a rise in PMT response cancelling a drop in scintillator light output. Following this (as the PMT response becomes constant over time) the response drops, due to a combination of scintillator decay and a steady temperature rise in the detector hall. 

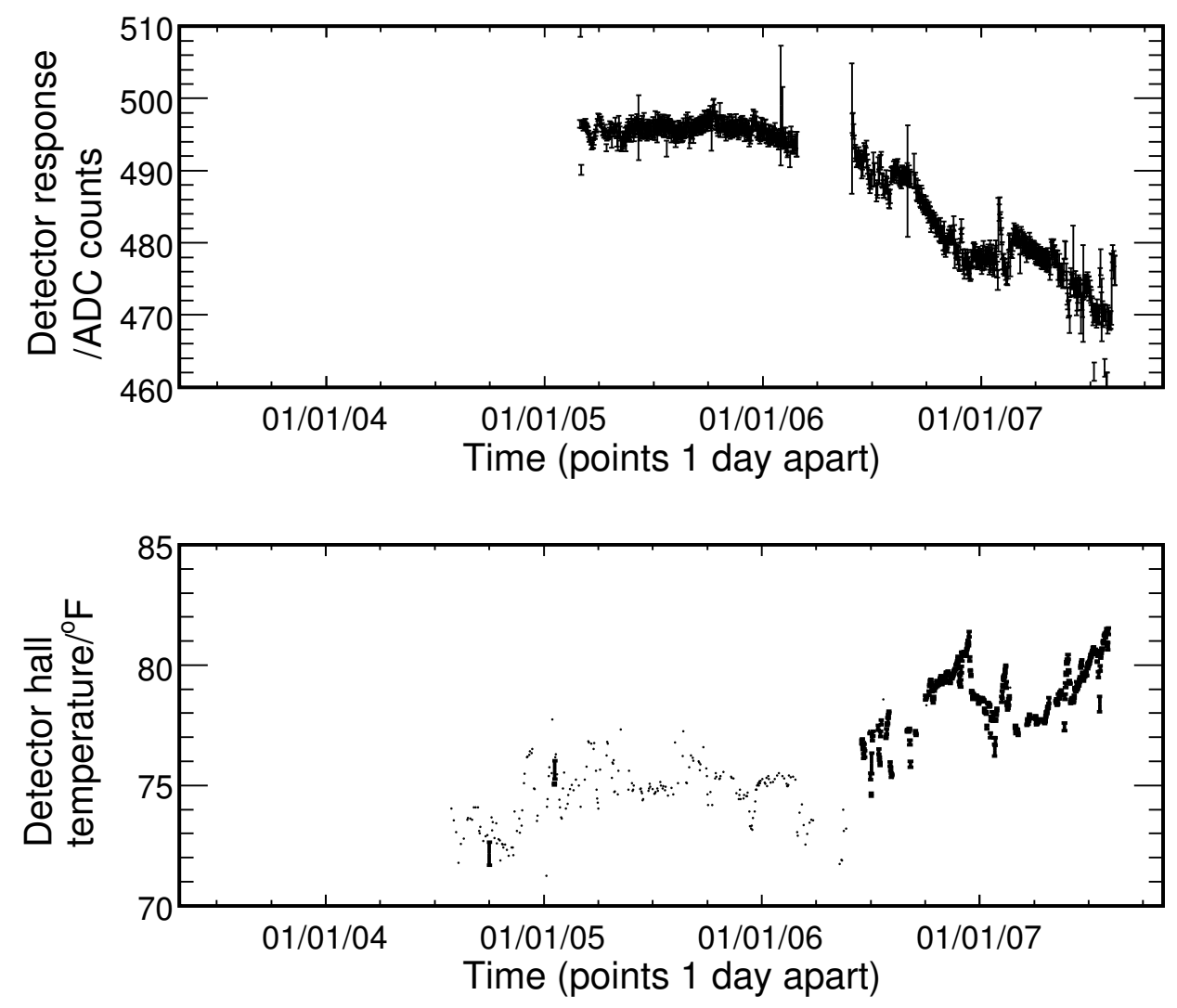

Figure 4.14: Top: the median plane-summed response of the near detector to cosmic muons over the full lifetime of the detector to date. Bottom: the mean daily temperature of the near detector hall. 

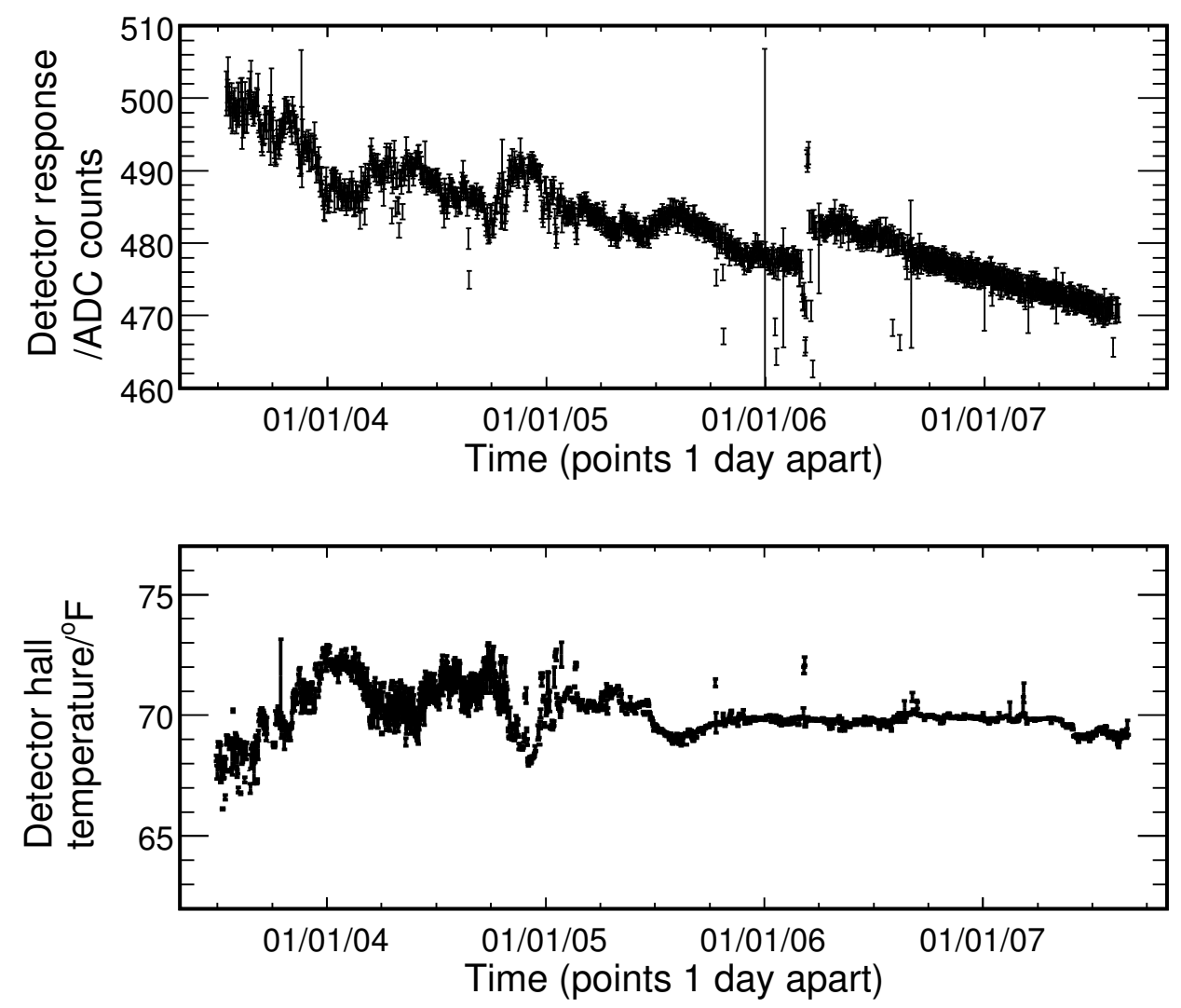

Figure 4.15: The set of plots equivalent to figure 4.14, here for the far detector. 


\subsubsection{Drift Constants}

To correct a pulse height $P_{Q \text { raw }}$ measured at a time $t$, an arbitrary reference date is chosen (currently 1st December 2005). The ratio of the median detector response on the reference day $\left(D\left(t_{0}\right)\right)$ and the median detector response at time $t(D(t))$ is used as a multiplicative correction to give the corrected pulse height:

$$
P_{Q \text { corr }}=\frac{D\left(t_{0}\right)}{D(t)} P_{Q \text { raw }}(t) .
$$

The ratio $D\left(t_{0}\right) / D(t)$ is referred to as a drift constant.

\subsubsection{Estimating Temperature Dependence in the Far De- tector}

A simple model was used to decouple the effect of temperature from the longterm decay in detector response. It is assumed that a $1^{\circ} \mathrm{F}$ absolute change in temperature causes the detector response to change by a factor $y$. For each day, the temperature differs from an arbitrary baseline temperature by $x^{\circ} \mathrm{F}$. The detector response that day is corrected by a factor $(1+x y)$. The new detector response as a function of time is then fitted to a straight line and the $\chi^{2}$ of this fit calculated. $x$ is varied until the best $\chi^{2}$ is obtained.

Any days with an anomalously low detector response are excluded from the straight line fit (this low response is due to detector rather than temperature effects). The fit was performed only up to 31st December 2006.

To deal with the jump in far detector response following the PMT voltage tuning, a factor is applied to all detector responses from 11th March 2006 onwards. The $\chi^{2}$ minimisation is performed jointly over this parameter and $x$.

The best fit in the far detector is for a drift in detector response, $x$, of $0.45 \%$ per ${ }^{\circ} \mathrm{F}$ with a PMT voltage tuning factor of 0.989 . The temperature-corrected 


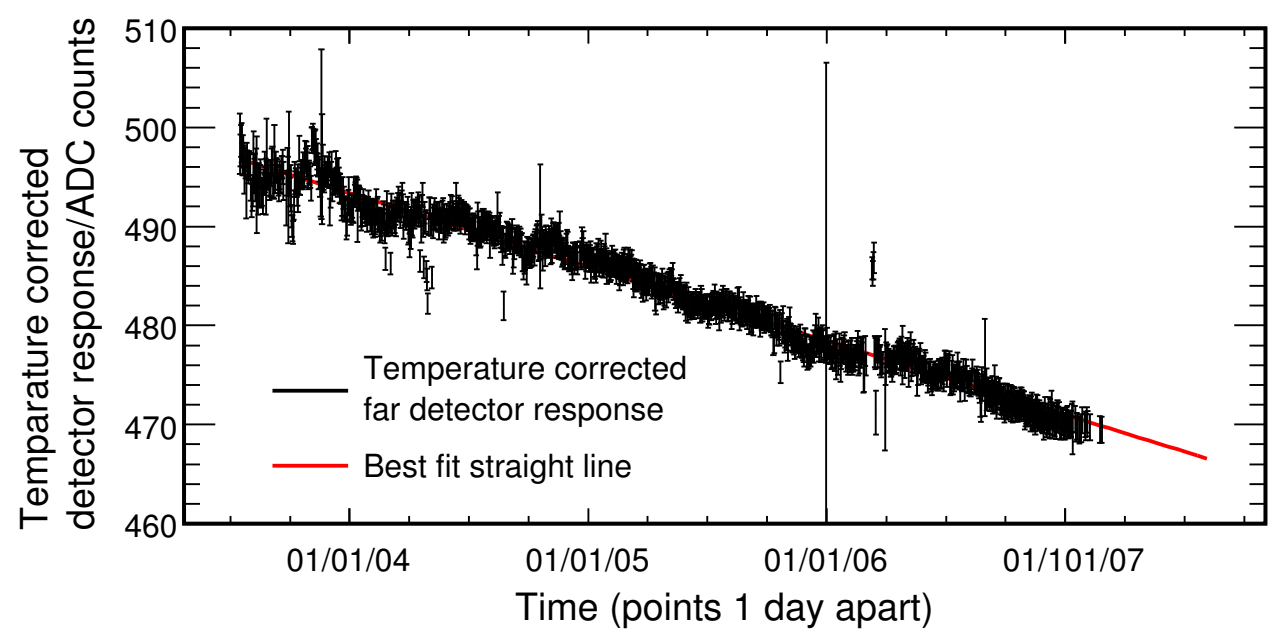

Figure 4.16: Black: the median plane-summed response of the far detector, corrected according to the temperature of the detector hall. Red: the best fit straight line to the temperature-corrected response.

detector response over time is shown in figure 4.16. The best fit straight line has a gradient of $-7.4 \mathrm{ADC}$ counts/year, implying a detector response decay of $\sim 1.5 \%$ year.

This method is not effective in the near detector for a combination of reasons. The rise in PMT gain slows over time, causing the long-term drift in response to differ from a straight line. The electronics are independently water-cooled, therefore less affected by detector hall temperatures. Furthermore, many more electronics changes are performed in the near detector compared to the far causing less stability in the detector response. With work, these effects could be decoupled to obtain a measurement of the scintillator temperature response. However this measurement, even at the far detector, is of academic interest only, not being used to correct the data. Therefore the measurement has not been performed at the near detector. Nevertheless, the fact that the far detector hall temperature is found to be strongly correlated with the measured detector response gives a valuable confirmation of the validity of the methods developed in this chapter. 


\subsubsection{Effect on a Physics Analysis}

The beam data analysed in this thesis was taken between the dates of 20th May 2005 and 5th February 2006, and from 1st June 2006 to 17th July 2007. Histograms of the drift constants in each detector for these periods are shown in figure 4.17 (the black histograms). The standard deviations of these histograms are 0.0182 and 0.0080 for the near and far detectors respectively. The drift calibration therefore removes a spread in the calorimetric hadronic energy measurement of $1.82 \%$ and $0.80 \%$ in the near and far detectors respectively.

To estimate a systematic error on the calorimetric energy scale due to this calculation, a histogram is made of drift constants calculated using the mean planesummed detector response rather than the median. These are the red histograms in figure 4.17. The difference between the means of the red and black histograms are taken as the systematic error.

The drift constants calculated from the median response have a mean of 1.0172 in the near detector and 1.0018 in the far. Calculating drift constants from the mean detector response give a mean constant of 1.0132 in the near detector and 1.0030 in the far. In the near detector, the systematic uncertainty on the calorimetric energy scale is therefore $0.4 \%$; in the far detector: $0.12 \%$.

\subsection{Summary}

The chain of calibrations applied to MINOS data for the purpose of measuring shower energies through calorimetry has been described, the need for a calibration to track the drift in overall detector response has been explained, and its relationship to the other calibrations shown.

The use of cosmic muons as the tool for this calibration has been justified, along with the process of selecting muons and the application of the necessary path-length correction, zero-plane reconstruction and fiducial cuts. 

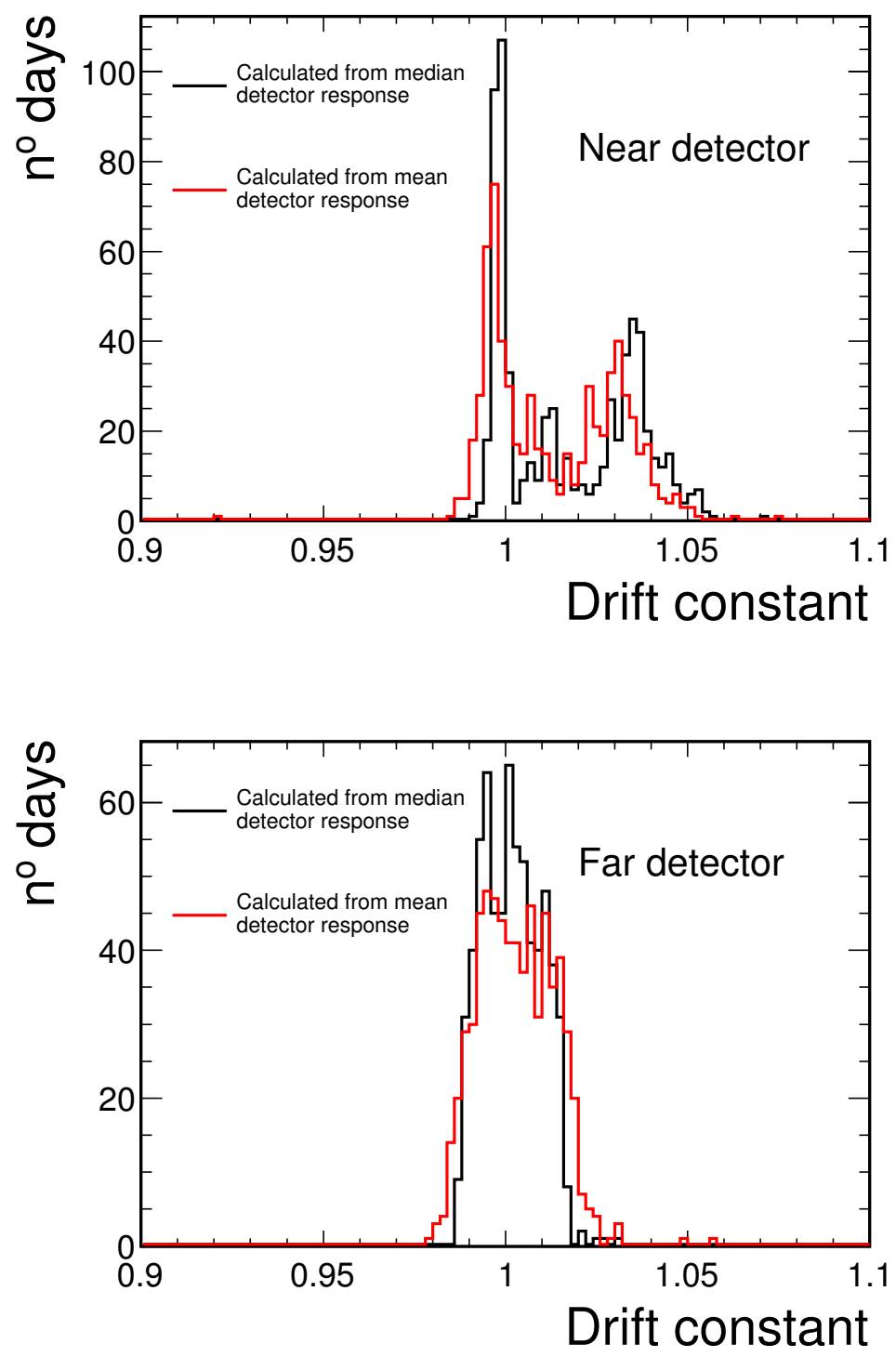

Figure 4.17: The drift constants applicable to the beam data for the near (top) and far (bottom) detectors. Constants are shown calculated from the median (black) and mean (red) detector response. 
The use of the daily median plane-summed pulse height to track detector response was found to be sensitive to changes in detector response caused by scintillator decay, PMT drift and environmental effects, whilst being robust against random fluctuations. The use of different software reconstruction versions was seen to require a further time-dependent correction factor.

The median plane-summed pulse height was used to calculate a daily correction factor to be applied to all reconstructed pulse heights in detector data. At the far detector the overall decay in detector response was decoupled from temperature effects to obtain an estimate of a $0.45 \%$ drift in response per $1^{\circ} \mathrm{F}$ and a steady decay in response of $1.5 \%$ per year. The effect of the calibration on the NuMI beam data analysed in this thesis was determined: a spread in calorimetric energy measurement of $1.82 \%$ and $0.80 \%$ was removed in the near and far detectors respectively; the systematic uncertainty on the calorimetric energy scale induced by the calibration was found to be $0.4 \%$ in the near detector and $0.12 \%$ in the far detector. 


\section{Chapter 5}

\section{Muon Charge Identification}

\section{$5.1 \quad$ Introduction}

Section 3.8.1 showed the topologies arising from neutrino interactions in the MINOS detectors. To identify charged current antineutrino interactions, the charge of the muon track must be determined: positive muons (bending away from the detector centre) signify $\bar{\nu}_{\mu}$ interactions; negative muons (bending towards the detector centre) signify $\nu_{\mu}$ interactions.

As discussed in section 3.8.1, a muon track can undergo multiple scattering or a hard scatter causing the charge to be misidentified. Additionally, a track can be reconstructed in a neutral current interaction, for example due to the presence of a charged pion, again mimicking a charged current $\bar{\nu}_{\mu}$ event topology. In this chapter, two new selection variables are developed to improve on the charge current $\bar{\nu}_{\mu}$ identification possible with existing analysis tools. In chapter 6 they will be incorporated into a full event selection. 

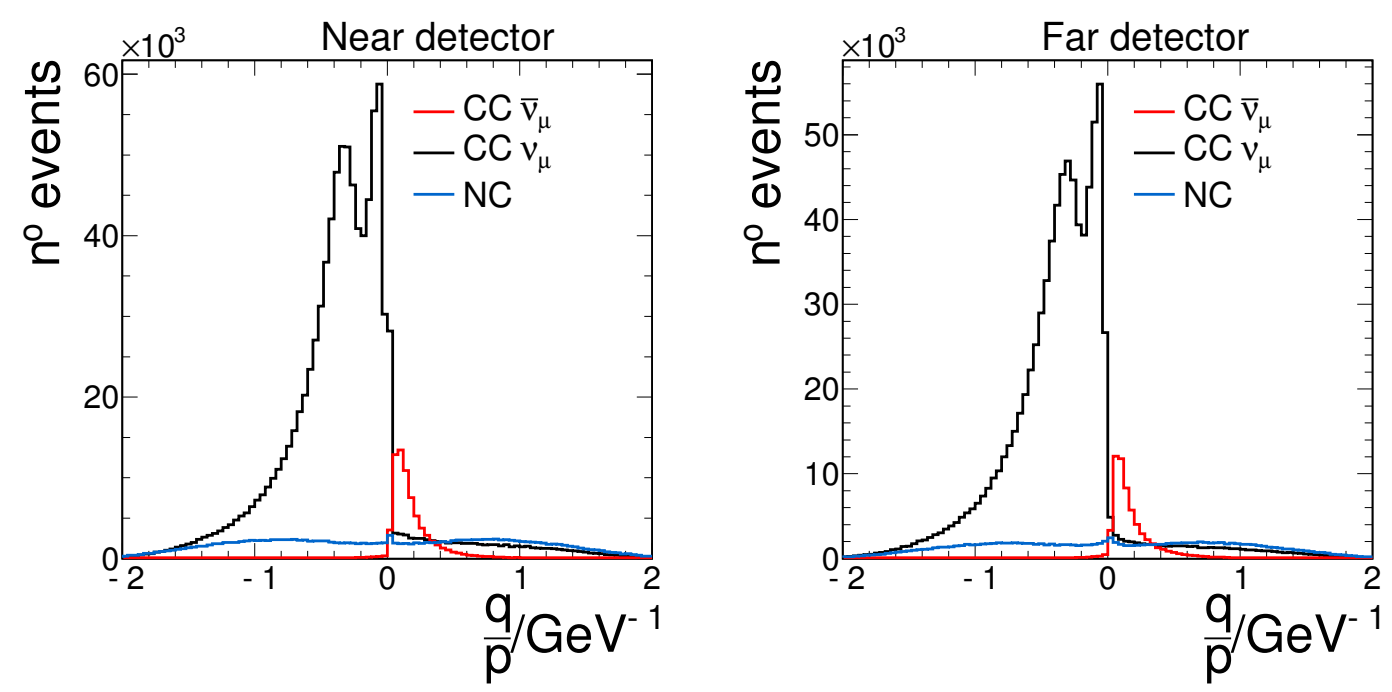

Figure 5.1: The reconstructed charge divided by momentum of tracks passing the preselection. Black: CC $\nu_{\mu}$ events; red: $\mathrm{CC} \bar{\nu}_{\mu}$ events; blue: neutral current events. Left: near detector; right: far detector.

\subsection{Reconstructed Charge}

The MINOS reconstruction uses a Kalman filter to reconstruct the charge and momentum of tracks (in which it assumes the tracks belong to muons) [157]. The variable returned is $\frac{q}{p}$ where $q$ is the reconstructed charge of the particle and $p$ the reconstructed momentum. After a simple preselection aimed at selecting good beam neutrino events (which will be discussed more fully in section 6.3.1), $\frac{q}{p}$ for all Monte Carlo events is shown, for both detectors, in figure 5.1 separated into $\mathrm{CC} \nu_{\mu}, \mathrm{CC} \bar{\nu}_{\mu}$ and other (predominantly NC) interactions. If events with $\frac{q}{p}<0$ (signifying a reconstructed $\mu^{-}$) are rejected, this removes, in the near and far detectors respectively, $89.2 \%$ and $93.0 \%$ of the $\mathrm{CC} \nu_{\mu}$ events, $3.3 \%$ and $3.8 \%$ of the CC $\bar{\nu}_{\mu}$ events, and $50.6 \%$ and $49.7 \%$ of the other (primarily NC) events.

Some tracks fail the fit performed with the Kalman filter (these events are given $\frac{q}{p}=0$ in figure 5.1). Of those events with $\frac{q}{p} \geq 0$, the fraction failing the fit are shown in figure 5.2. These events are rejected. In the near and far detector respectively this rejects $24.9 \%$ and $2.2 \%$ of the wrong-sign $\mathrm{CC} \nu_{\mu}$ events, $0.1 \%$ and $0.02 \%$ of the $\mathrm{CC} \bar{\nu}_{\mu}$ events and $1.0 \%$ and $0.6 \%$ of the $\mathrm{NC}$ events. As this is the 

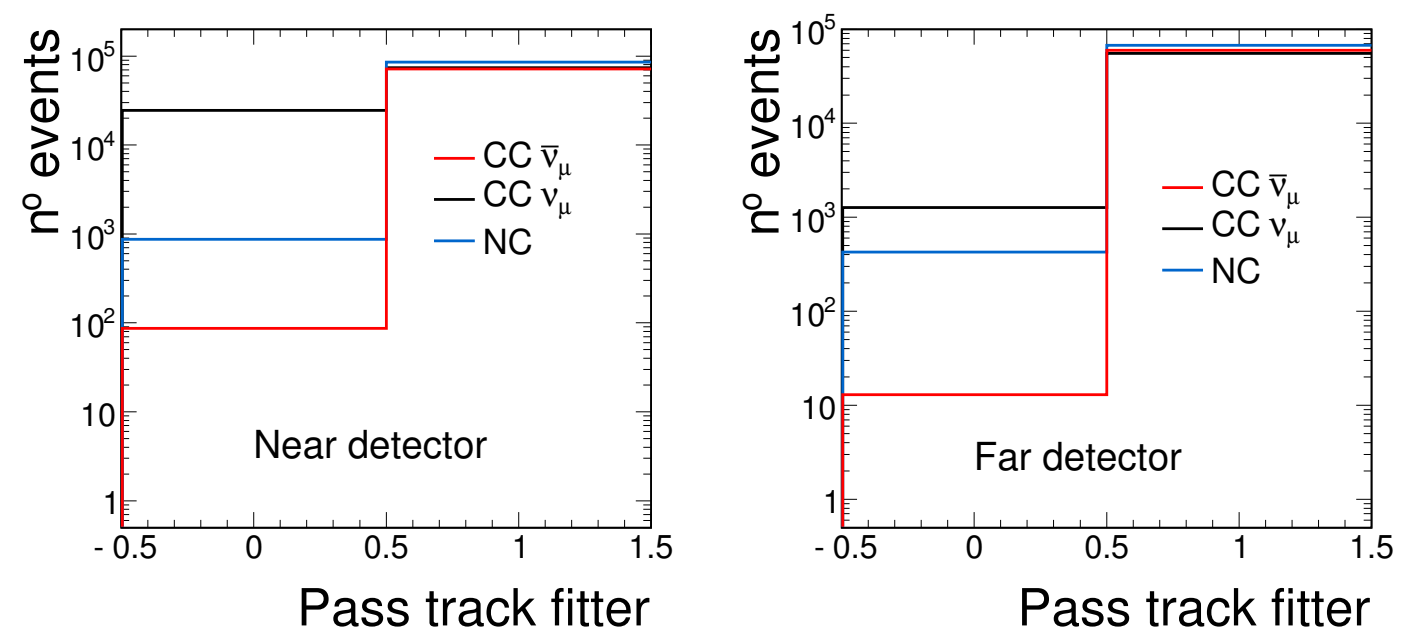

Figure 5.2: Events failing (0) and passing (1) the track fit which pass the preselection and have $\frac{q}{p} \geq 0$, as for figure 5.1. Left: near detector; right: far detector.

sample of tracks assigned a positive charge, the CC $\nu_{\mu}$ events in this sample have had the incorrect charge assigned. This is therefore a sample of 'bad' tracks and so would be expected to fail the track fitter more often than the sample of 'good' $\mathrm{CC} \nu_{\mu}$ events.

The energy spectra of the remaining events are shown in figure 5.3, along with the spectrum of $\mathrm{CC} \bar{\nu}_{\mu}$ events remaining after preselection only. Also shown are the efficiency $\mathcal{E}$ and purity $\mathcal{P}$ where, for each energy bin,

$$
\begin{aligned}
& \mathcal{E}=\frac{\mathrm{N}^{\mathrm{o}} \mathrm{CC} \bar{\nu}_{\mu} \text { events remaining after the cuts }}{\mathrm{N}^{\mathrm{o}} \mathrm{CC} \bar{\nu}_{\mu} \text { events before the cuts }}, \\
& \mathcal{P}=\frac{\mathrm{N}^{\mathrm{o}} \mathrm{CC} \bar{\nu}_{\mu} \text { events remaining after the cuts }}{\text { Total } \mathrm{n}^{\mathrm{o}} \text { events remaining after the cuts }} .
\end{aligned}
$$

Note that the denominator of equation 5.1 is only those events remaining after preselection. The aim of this thesis is to effectively select charged current $\bar{\nu}_{\mu}$ events: the requirement of at least one track is considered a reconstruction issue, and considered beyond the scope of the thesis.

There is a considerable background of charged current $\nu_{\mu}$ and neutral cur- 

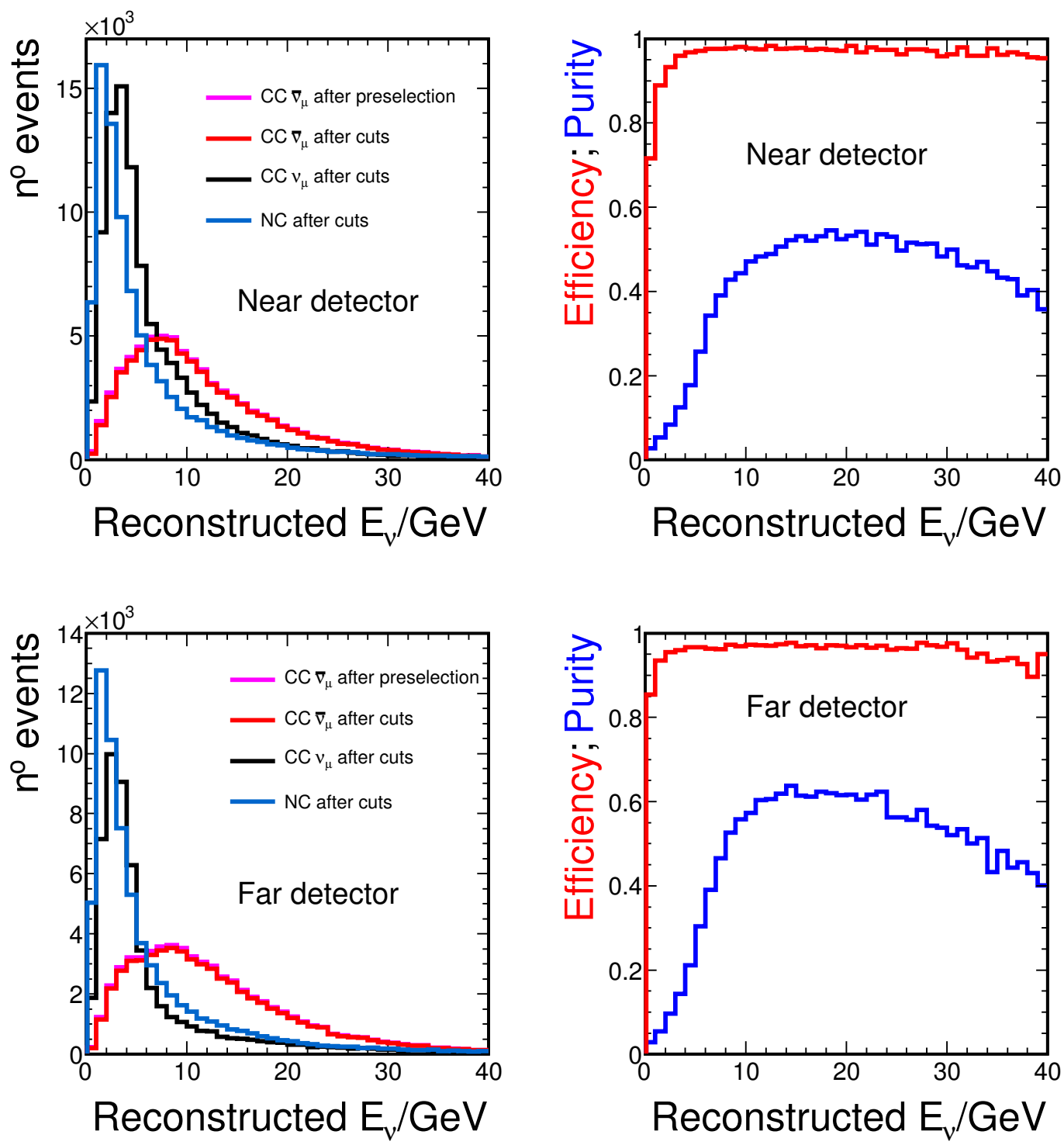

Figure 5.3: Left: reconstructed energy spectra of neutrino interactions passing the preselection and the track fit, with $\frac{q}{p}>=0$ (red, black, blue); and the reconstructed energy spectrum of all CC $\bar{\nu}_{\mu}$ interactions which pass the preselection (magenta). Right: the corresponding efficiency (red) and purity (blue) of the $\mathrm{CC} \bar{\nu}_{\mu}$ selection, as defined in equations 5.1 and 5.2. Top: near detector. Bottom: far detector. 
rent events remaining: the $\bar{\nu}_{\mu}$ signal is heavily dominated by the background. (At very high energies the purity of the sample worsens as the muon tracks become straighter and their direction of curvature harder to identify.) To perform a successful physics analysis with the antineutrinos, this background must be significantly reduced. Chapter 6 shows how a number of variables can be used to produce an effective $\bar{\nu}_{\mu}$ selection; two of those variables are developed in the remainder of this chapter.

\subsection{Majority Curvature}

The reconstruction software can assign a wrong sign $\frac{q}{p}$ to tracks, for example through a hard scatter or multiple scattering as discussed in section 3.8.1. A technique has been developed, for this thesis, to improve the charge reconstruction in such cases.

A segment of $n$ consecutive (in ascending plane number) hits is taken from the track, beginning with a segment starting on the first hit. Two parabolas are fit to this segment: one to the $|x|$ verses $z$ co-ordinates of the strips, the other to the $|y|$ verses $z$ co-ordinates (see figure 5.4). These parabolas have the form

$$
(|x|,|y|)=a z^{2}+b z+c
$$

where $a$ and $b$ are parameters to be fitted.

The $(x, y)$ co-ordinate system is centred on the coil hole. The use of the modulus of the $x$ and $y$ co-ordinates means the direction of curvature of the track is given by the second derivative of the parabola: $2 a .2 a>0$ implies a positive track (signifying a $\bar{\nu}$-like event). $2 a<0$ implies a negative track (signifying a $\nu_{\mu}$-like event).

Starting from the second hit in the track, the next $n$ strips are taken and treated 


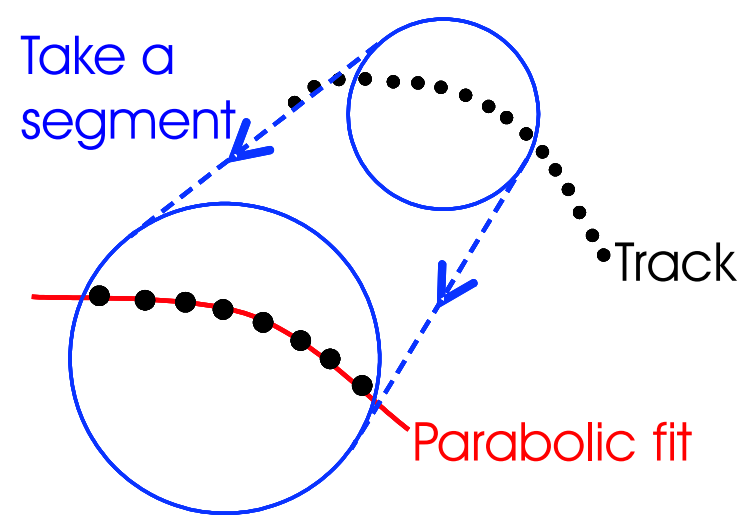

Figure 5.4: An illustration of the calculation of the majority curvature of a track. A segment is taken from the start of a track. A parabolic fit is performed separately on the $x$ and $y$ against $z$ co-ordinates of the hits to give the direction of curvature (positive or negative) of the track. Moving along one hit at a time, this fit is performed on successive segments. The numbers of positively and negatively curving track segments are compared to form the majority curvature variable.

in the same way, and so on until the last strip of the track has been included in a segment. For each co-ordinate view ( $x$ or $y$ ), the number of $2 a>0$ segments is totalled up and designated $P_{x, y}$. Similarly the number of $2 a<0$ segments is designated $N_{x, y}$. Any segments which cross an $x$ or $y$ axis are not counted in the respective totals, as at this point the curvature due to the magnetic field changes sign, causing the segment to have a wrong-signed kink in the modulus of the co-ordinate (figure 5.5).

The majority curvature, $C_{x, y}$, of each view of the track is then defined:

$$
C_{x, y}=\left\{\begin{aligned}
10 & \text { if } N_{x, y}=0 \\
-10 & \text { if } P_{x, y}=0 \\
\ln \left(\frac{P_{x, y}}{N_{x, y}}\right) & \text { otherwise }
\end{aligned}\right.
$$

Whichever view of $C_{x, y}$ has the largest magnitude is taken as the majority curvature for the track:

$$
C_{M}= \begin{cases}C_{x} & \text { if }\left|C_{x}\right|>\left|C_{y}\right| \\ C_{y} & \text { if }\left|C_{y}\right|>\left|C_{x}\right|\end{cases}
$$




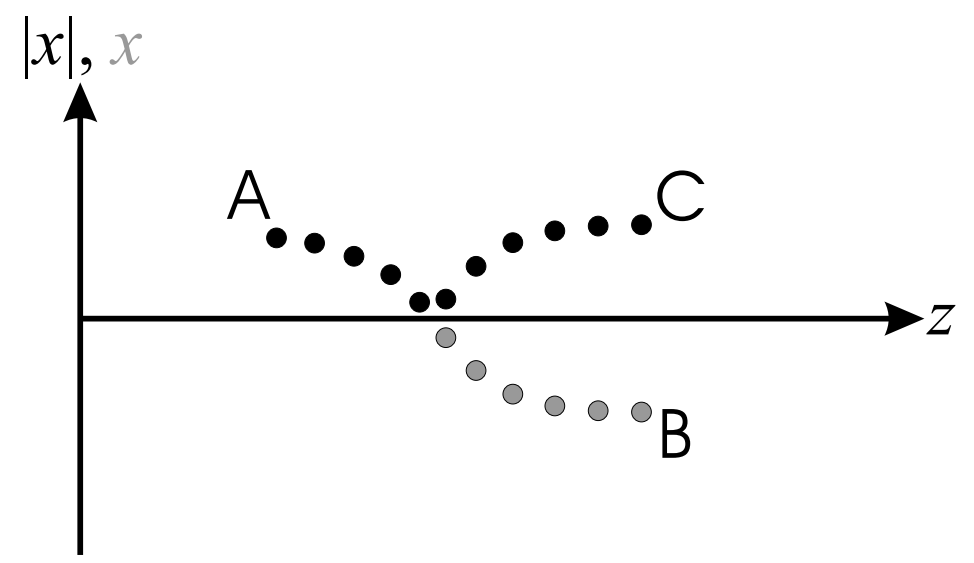

Figure 5.5: The dots represent hit strips along a track in the detector. The detected track runs from $A$ to $B$. In calculating the majority curvature, the modulus of the $x$ co-ordinate $|x|$ is used. In this representation, the track runs from $A$ to $C$, causing a wrong-signed kink where the track reaches the $x$-axis. Segments containing this kink are not used in the calculation of majority curvature.

The $x$ and $y$ views are used rather than $u$ and $v$ as in the near detector the beam passes through on one side of the coil hole. Here the magnetic field is primarily vertical, so the most pronounced curvature will usually be in the $x$-direction. For consistency, the same treatment is followed in the far detector.

The length of the track segments used in the fit was optimised by maximising the (efficiency $\times$ purity) of the selected antineutrino sample. The best result was found using segments of seven detector planes. If, after the calculation, $C_{M}=$ 0 , the procedure is repeated with differing segment lengths. Through a similar optimisation, eight-plane segments are next used, then steadily decreasing segment lengths (six, five, ...) down to three planes. At this point, if a non-zero value of $C_{M}$ has not been obtained, the event is rejected. For tracks shorter than eight planes, the first segment length used is equal to the track length, followed again by lengths steadily decreasing down to three planes.

$C_{M}$ is shown for all events passing the preselection and the track fit, and with $\frac{q}{p}>0$, in figure 5.6. The default values of $C_{M}= \pm 10$ are visible. These belong primarily to lower energy (primarily $\mathrm{CC} \nu_{\mu}$ and NC) events as the shorter track leaves more chance of no segments having one of the directions of curvature 

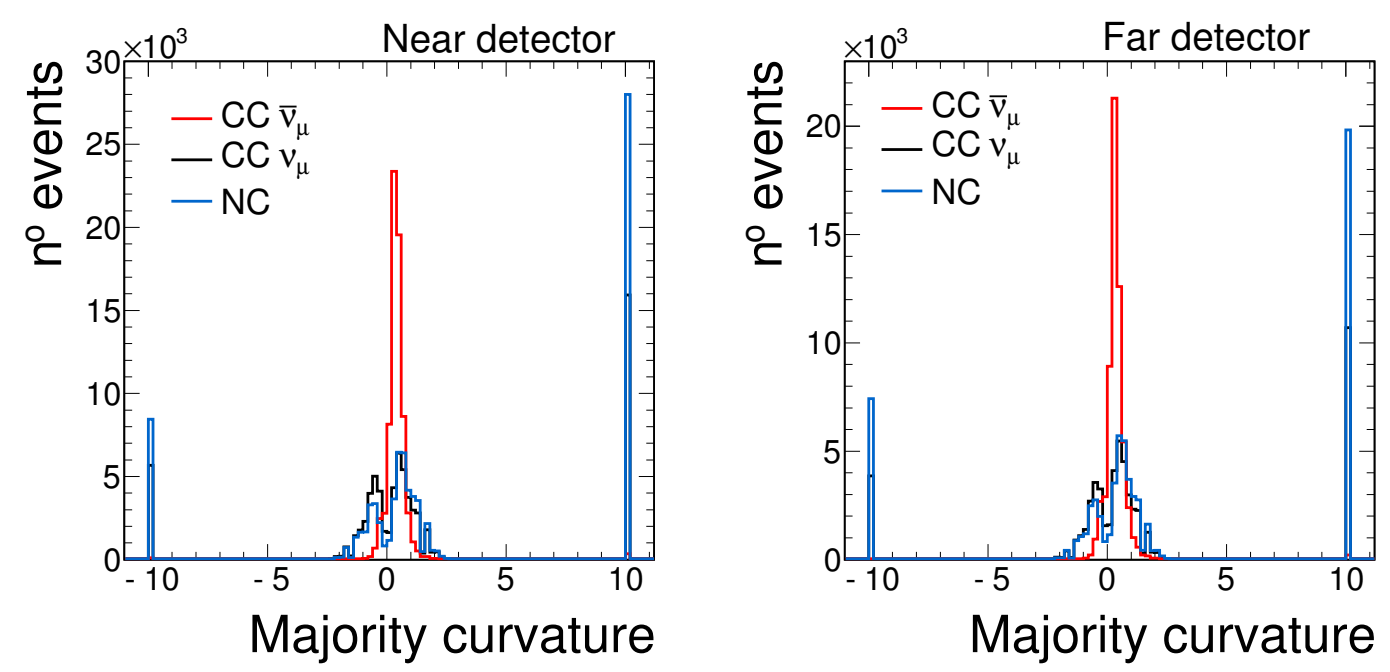

Figure 5.6: Majority curvature of all events passing the preselection and track fit, and with $\frac{q}{p} \geq 0$, as for figure 5.1. Left: near detector; right: far detector.

(particularly if a track is so short that only one segment is used in the fit).

Events with $C_{M}<0$ are removed. In the near and far detector respectively this rejects $37.1 \%$ and $34.4 \%$ of the wrong-sign CC $\nu_{\mu}$ events, $8.9 \%$ and $11.5 \%$ of the CC $\bar{\nu}_{\mu}$ events and $28.2 \%$ and $28.6 \%$ of the NC events. The new variable is therefore seen to be a valuable addition to the Kalman filter of the standard reconstruction, effectively removing one third of the misidentified charged current $\nu_{\mu}$ events with a much smaller loss in $\bar{\nu}_{\mu}$ selection efficiency.

Figure 5.7 shows as a function of track length the fraction of those events passing the aforementioned cuts which are removed by the majority curvature cut. The majority curvature cut has its greatest background-removal effectivity for longer tracks, suggesting it is most effective at identifying quite straight (high energy) tracks which have undergone a scatter such as to fake a positive curvature.

\subsection{Track Jitter}

An extension of the majority curvature calculation allows sensitivity not only to the charge of a track, but also to the confidence with which we know that charge. 

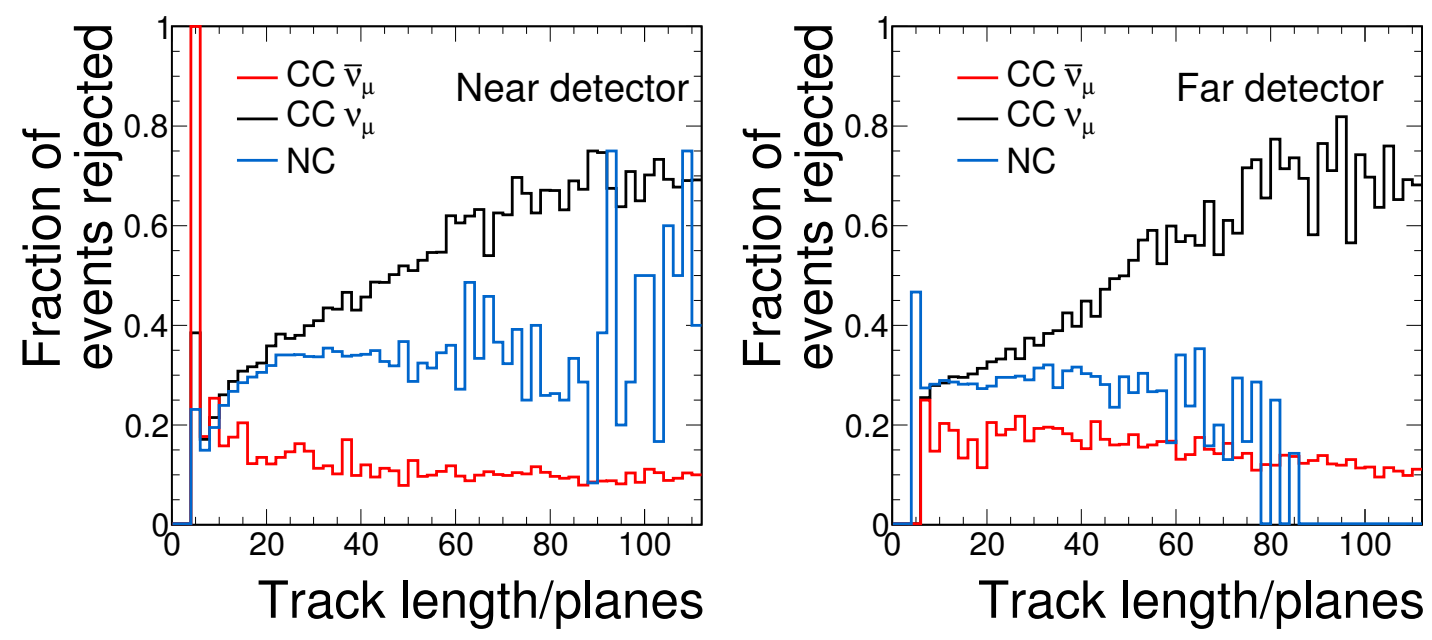

Figure 5.7: Of the events which pass the preselection and track fit, and have $\frac{q}{p} \geq 0$, the graphs show the fraction (as a function of track length) which have a negative majority curvature, as for figure 5.1. Left: near detector; right: far detector.

This will further eliminate muons which have scattered, or charged pions which are more prone to hadronic interactions in the detector. 'Fake' tracks across hadronic showers (figure 3.19) should also prove more ambiguous in charge.

The less a particle scatters, the smoother will be the curvature of its track. Thus when calculating majority curvature the curvature of the successive segments, $2 a$, will vary slowly. The curvature of a track which scatters heavily will vary more quickly between successive segments. A measure of the track's jitter, $J$, can be obtained by taking the average change in curvature between successive segments. For a track with $N$ segments, labelled $i$ (where the first segment is $i=0)$,

$$
J=\frac{\sum_{i=1}^{N-1}\left|2 a_{i}-2 a_{i-1}\right|}{N}
$$

Tracks so short that only one segment is used for the calculation are assigned $J=0$.

$J$ is shown in figure 5.8 for events passing preselection, with $\frac{q}{p}>0$ and $C_{M}>0$. Good separation is obtained for both neutral current events and wrong-sign CC $\nu_{\mu}$ events, the reconstructed tracks of which will not arise from smoothly curving 

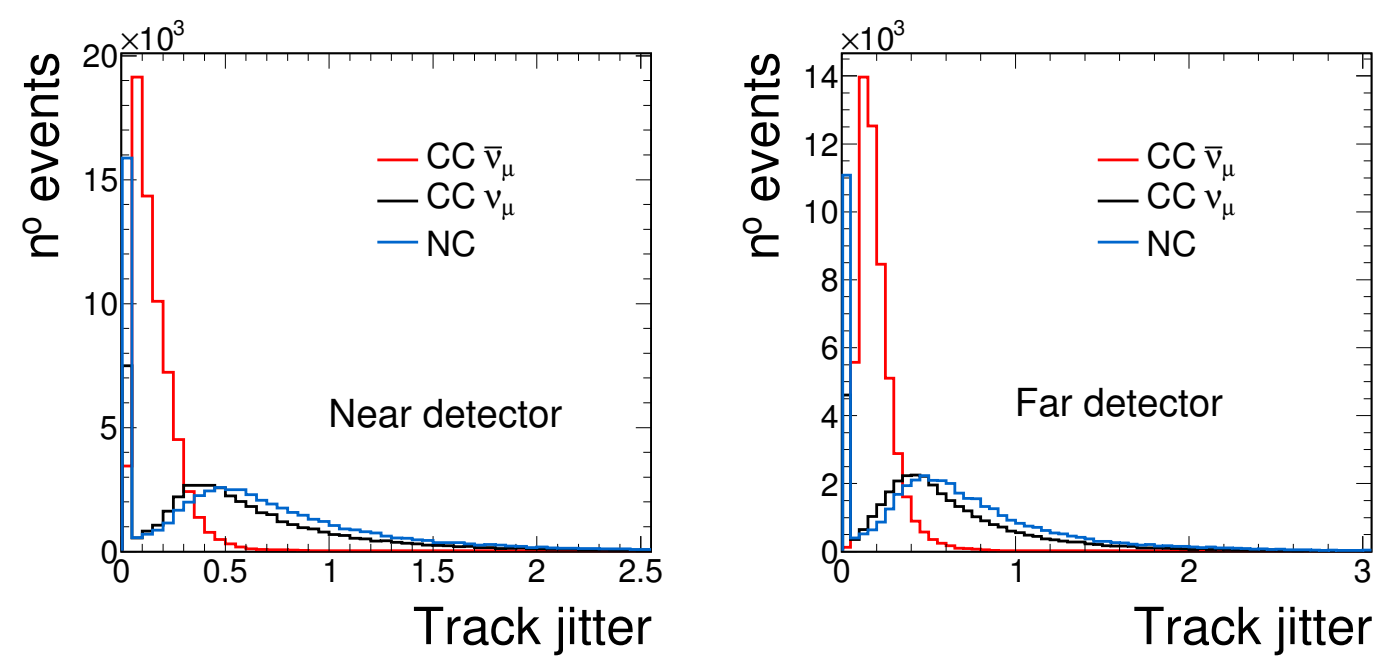

Figure 5.8: Track jitter for events passing the preselection, reconstructed charge and majority curvature cuts, as for figure 5.1. Left: near detector; right: far detector.

muons. The figures show that up to half the background falls at values of $J$ higher than the majority of the background. However, in chapter 6 it will be seen that the power of this variable can be further improved through its combination with a pre-existing selection tool.

\subsection{Summary}

The reconstructed charge of a muon track is the primary signature of a charged current $\bar{\nu}_{\mu}$ interaction. The standard MINOS reconstruction provides a method of performing such charge identification, but large backgrounds of misidentified charged current $\nu_{\mu}$ and neutral current events remain. In this chapter, a variable (majority curvature) has been developed which splits tracks into successive, overlapping segments and performs parabolic fits to find the direction of curvature of these segments. The track is assigned a charge according to the direction in which the majority of these segments are seen to curve. This process has also been extended to look at the confidence with the which the charge is measured, forming a variable called track jitter. This is formed by looking at the average change in 
curvature between successive track segments. Both new variables are successful in reducing the level of background contaminating the charged current $\bar{\nu}_{\mu}$ sample and will be combined into a full selection in chapter 6 . 


\section{Chapter 6}

\section{Charged Current $\bar{\nu}_{\mu}$ Event Selection}

\subsection{Introduction}

In section 3.8.1, the topologies of the various neutrino interactions in the MINOS detectors were described. In chapter 5, two new variables were developed to identify the charge of a muon track, a positive track being the primary signature of a charged current $\bar{\nu}_{\mu}$ event. In this chapter, a full charged current $\bar{\nu}_{\mu}$ selection will be developed to produce an antineutrino sample pure enough to be used in a physics analysis.

It is important that the $\mathrm{CC} \nu_{\mu}$ background is eliminated as far as possible, and any remaining background well measured, as the oscillation of misidentified muon neutrinos will mask the oscillations of the muon antineutrinos. Additionally, figure 3.5 has shown that muon neutrinos dominate muon antineutrinos in the beam. Therefore there is a very large source of $\mathrm{CC} \nu_{\mu}$ background: any systematic uncertainties affecting muon charge identification could cause large changes in the number of misidentified CC $\nu_{\mu}$ interactions, particularly important for a $\bar{\nu}_{\mu}$ appearance analysis in which a wrongly measured CC $\nu_{\mu}$ background will eas- 
ily imitate the conjectured signal. To guard against these effects, a high purity selection has been developed.

\subsection{Detector Noise}

Very little non-beam background affects this analysis. Most detector-related noise (for example any light injection which has mistakenly been left in the dataset) forms clumps of detector response with a shower-like topology, therefore being removed in the same way as $\mathrm{NC}$ events. The only non-beam source of tracks is cosmic muons. These are removed by requiring selected events to be within $50 \mu \mathrm{s}$ (far detector) or $13 \mu \mathrm{s}$ (near detector) of a beam spill ${ }^{1}$, and with an extra cut in the far detector requiring selected tracks to have an angle $\theta$ with the beam direction such that $\cos \theta>0.6$.

Beam neutrinos interacting in the rock can produce events which spill into the detector: such events are dealt with by fiducial cuts detailed in section 6.3.1. A possibility which would not be mitigated by such cuts is that a neutron produced by a rock event travels into the centre of the detector before interacting. However, this would produce a short, NC-like event topology which will be removed by the cuts aimed at eliminating NC events. If such an event was to produce a highenergy charged pion track, this generally gives a track less smoothly curving than a muon hence will be dealt with by track quality cuts which will be discussed later.

\subsection{Selection in the Near and Far Detectors}

The far detector is larger than the near. Therefore the defocused $\mu^{+}$tracks travel further in the far detector before exiting through the sides or back, providing a longer section of track to enable identification. A much purer selection can be

\footnotetext{
${ }^{1}$ There is no need for the far detector timing cut to be weaker than in the near detector; these values are merely historical and work is underway to validate a tighter far detector cut.
} 
therefore be obtained in the far detector without significant loss of $\bar{\nu}_{\mu}$ identification efficiency.

The selection philosophy taken in this thesis has two components. Firstly, the selection purity is tuned to be as high as reasonably possible (whilst maintaining a reasonable selection efficiency). This minimises the effect of systematic uncertainties: so little background remains that a background mismodelling is unlikely to affect the final analysis. Secondly, identical selections will be used in the near and far detectors so that the two-detector nature of MINOS can be exploited, relying on many systematic uncertainties to affect both detectors identically and therefore cancel out.

\subsubsection{Preselection}

The main feature used to identify a $\mathrm{CC} \bar{\nu}_{\mu}$ event is the $\mu^{+}$track. Therefore all events with no reconstructed tracks are eliminated. For all events with more than one reconstructed track the longest is chosen as the primary track. This is taken to be the muon track.

Neutrinos from the beam may interact in the rock surrounding the detector and the resulting particles spill into the detector; alternatively a neutrino may interact near the edge of the detector and the resulting particles be lost outside the detector. As only a fraction of these events are recorded, the energy of the neutrino cannot be well measured. A fiducial cut is therefore applied to events based on the position of the start of the primary track (track vertex). In the near detector, the track vertex is required to be within a cylinder of radius $80 \mathrm{~cm}$ centred on the neutrino beam centre. The front face of this cylinder falls such as to include the 14th plane from the front of the detector; the back face is such that the 68th plane is included and the 69th excluded. The back of this cylinder is then a little over $3 \mathrm{~m}$ in front of the back of the fully instrumented region of the 
detector, ensuring good containment of hadronic showers, allowing the hadronic energy to be well measured through calorimetry. The outside of the cylinder is never less than $35 \mathrm{~cm}$ from the detector edge.

With the same justification, the track vertex in the far detector is required to be within a cylinder of radius $3.742 \mathrm{~m}$ centred on the coil hole. This cylinder runs inclusively from the 4 th to the 239 th detector plane, then from 253 rd to the 464 th plane (the gap in the middle cutting out events with showers that may spill into the gap between supermodules). Additionally the track vertex must not fall in a cylinder of radius $40 \mathrm{~cm}$ centred on the coil hole, in order to remove events losing some of the hadronic shower or a large fraction of muon track into the coil hole. The outside of the larger cylinder is never less than $25 \mathrm{~cm}$ from the detector edge.

\subsubsection{Muon Charge Identification}

Muon charge identification was discussed in chapter 5. In this chapter, all events with $\frac{q}{p}>0$ and $C_{M}<0$ will rejected, along with those failing the track fitter. The remaining event sample will form the basis for the rest of the chapter.

\subsubsection{Neutral Current Background Removal}

\section{Neutral Current PID}

A method of identifying events from NC interactions was developed for the first MINOS neutrino oscillation analysis $[158,159]$. Three variables are combined to form a single particle identification (PID) variable, $P_{D}$. These variables are the event length (in planes), the fraction of the event's total pulse height which is in the reconstructed track, and the average pulse height per plane in tracked hits. From the Monte Carlo, the distributions of these variables are obtained separately for the CC and NC events. The distributions are normalised to act as probability density functions: for each data event, the content of the corresponding bin in the 

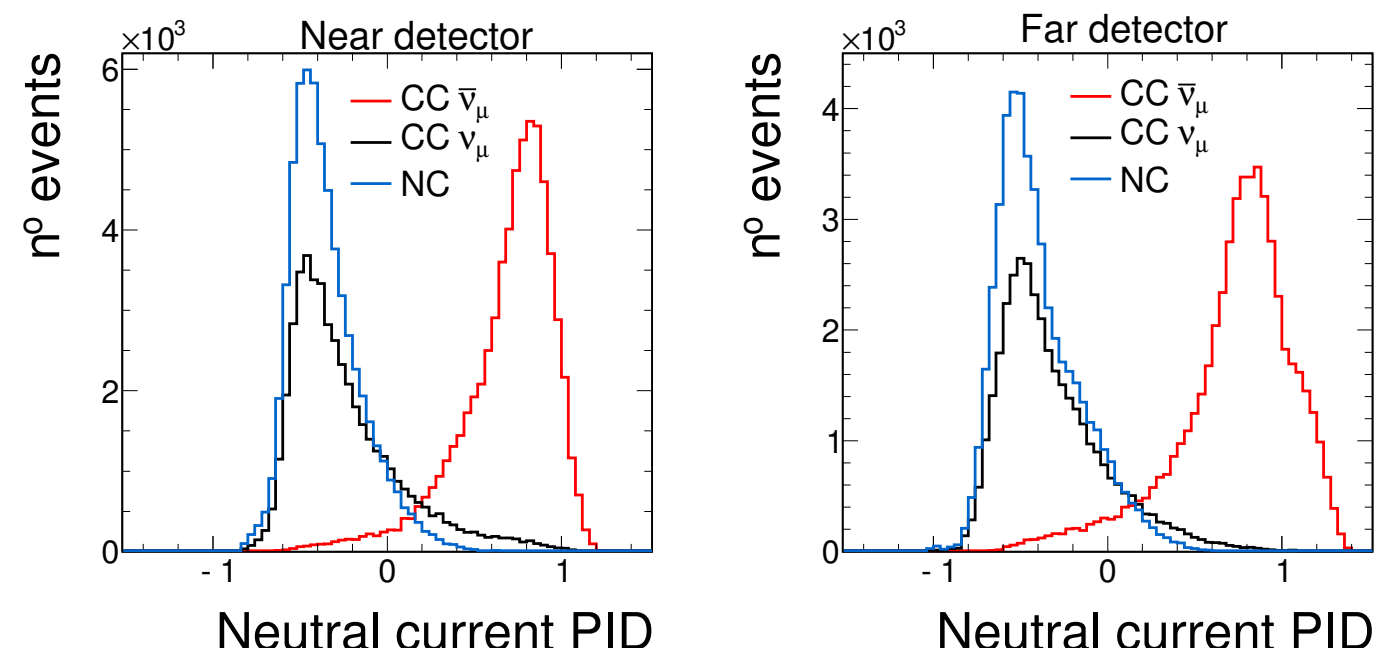

Figure 6.1: The neutral current PID (equation 6.1) for events passing the preselection, reconstructed charge and majority curvature cuts, as for figure 5.1. Left: near detector; right: far detector.

$\mathrm{CC}$ and $\mathrm{NC}$ histograms is $f_{i(\mathrm{CC}, \mathrm{NC})}$ where $i$ labels each of the three variables. The product over the three $\mathrm{CC}$ or $\mathrm{NC}$ histograms produces two variables:

$$
P_{\mathrm{CC}, \mathrm{NC}}=\prod_{i=1}^{3} f_{i,(\mathrm{CC}, \mathrm{NC})} \text {. }
$$

These variables are combined to form the PID:

$$
P_{D}=-\left(\sqrt{-\ln P_{\mathrm{CC}}}-\sqrt{-\ln P_{\mathrm{NC}}}\right) .
$$

A higher value of $P_{D}$ implies a more CC-like event.

$P_{D}$ is shown in figure 6.1 for all events passing the preselection and with $\frac{q}{p}>0$ and $C_{M}>0$. The MINOS neutrino oscillation analysis chose to reject events with $P_{D}<-0.2$ in the far detector and $P_{D}<-0.1$ in the near detector. However figure 6.1 shows that for a $\mathrm{CC} \bar{\nu}_{\mu}$ selection, better purity is achieved by rejecting events below a higher value of $P_{D}$ : this removes $\mathrm{CC} \nu_{\mu}$ background as there are many more $\nu_{\mu}$ events than $\bar{\nu}_{\mu}$ events in the low energy region where events look more NC-like; furthermore $\mathrm{CC} \bar{\nu}_{\mu}$ events have, on average, a lower $y$ (fraction of 
the neutrino energy given to the hadronic shower) than $\mathrm{CC} \nu_{\mu}$ events so look more CC-like.

However, making a straightforward cut on $P_{D}$ is not the best thing to do. Figure 6.2 shows $P_{D}$ as a function of reconstructed energy for events with $\frac{q}{p}>0$ and $C_{M}>0$. At higher energies the $\mathrm{CC} \bar{\nu}_{\mu}$ events have a more NC-like $P_{D}$ and would be needlessly rejected (there is little NC contamination in this region). This occurs as, once the (defocused) $\mu^{+}$track leaves the detector, as the neutrino energy increases the amount of pulse height in the track remains the same but the hadronic shower increases in size. This higher shower-to-track ratio gives the event a more NC-like $P_{D}$.

\section{Combining PID with Track Jitter}

Figure 6.3 shows a two-dimensional histogram of $J$ against $P_{D}$ for Monte Carlo events. Those events with a high track jitter are more likely to be NC events, or CC $\nu_{\mu}$ events with misidentified charge. Therefore as $J$ increases, a harsher cut on $P_{D}$ is made. The magenta line in figure 6.3 shows the cut: events above and to the left of the line are removed. Mathematically, events are rejected which have $P_{D}<0$ or $J>0.5$ or $J>0.59 P_{D}+0.2$.

\subsubsection{Wrong-Sign Charged Current Background Removal}

\section{Track Reconstruction Uncertainty}

The track reconstruction software discussed in sections 3.8.3 and 5.2 returns an uncertainty on its calculated value of $\frac{q}{p}$, which is labelled $\sigma\left(\frac{q}{p}\right)$. This is shown for all events passing the preceding cuts in figure 6.4. This uncertainty is obtained from the covariance matrix: a matrix used in the Kalman filter to describe the uncertainty in the fitted track at each detector plane. The uncertainty used in this selection is the final uncertainty at the track vertex. 

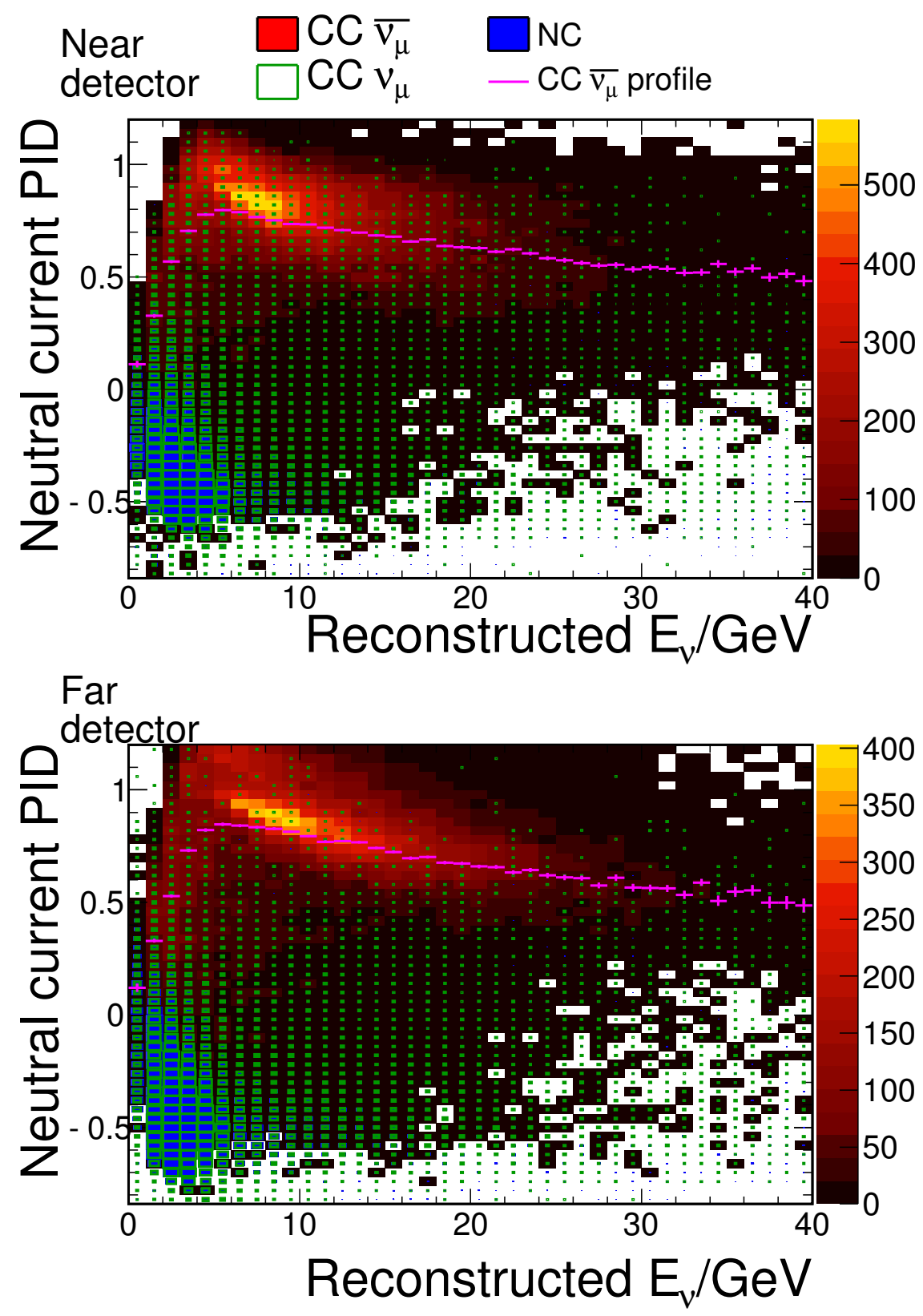

Figure 6.2: The neutral current PID (equation 6.1) as a function of reconstructed neutrino energy for the set of events in figure 6.1. The coloured histogram ranging from black through red to yellow (following the colour scale on the right) shows the $\mathrm{CC} \bar{\nu}_{\mu}$ events. The green and blue squares represent respectively $\mathrm{CC} \nu_{\mu}$ events and all other (primarily NC) events, larger squares representing higher numbers of events. The magenta line is a profile of the $\mathrm{CC} \bar{\nu}_{\mu}$ histogram, showing the average neutral current PID for each bin in reconstructed energy. Top: near detector; bottom: far detector. 

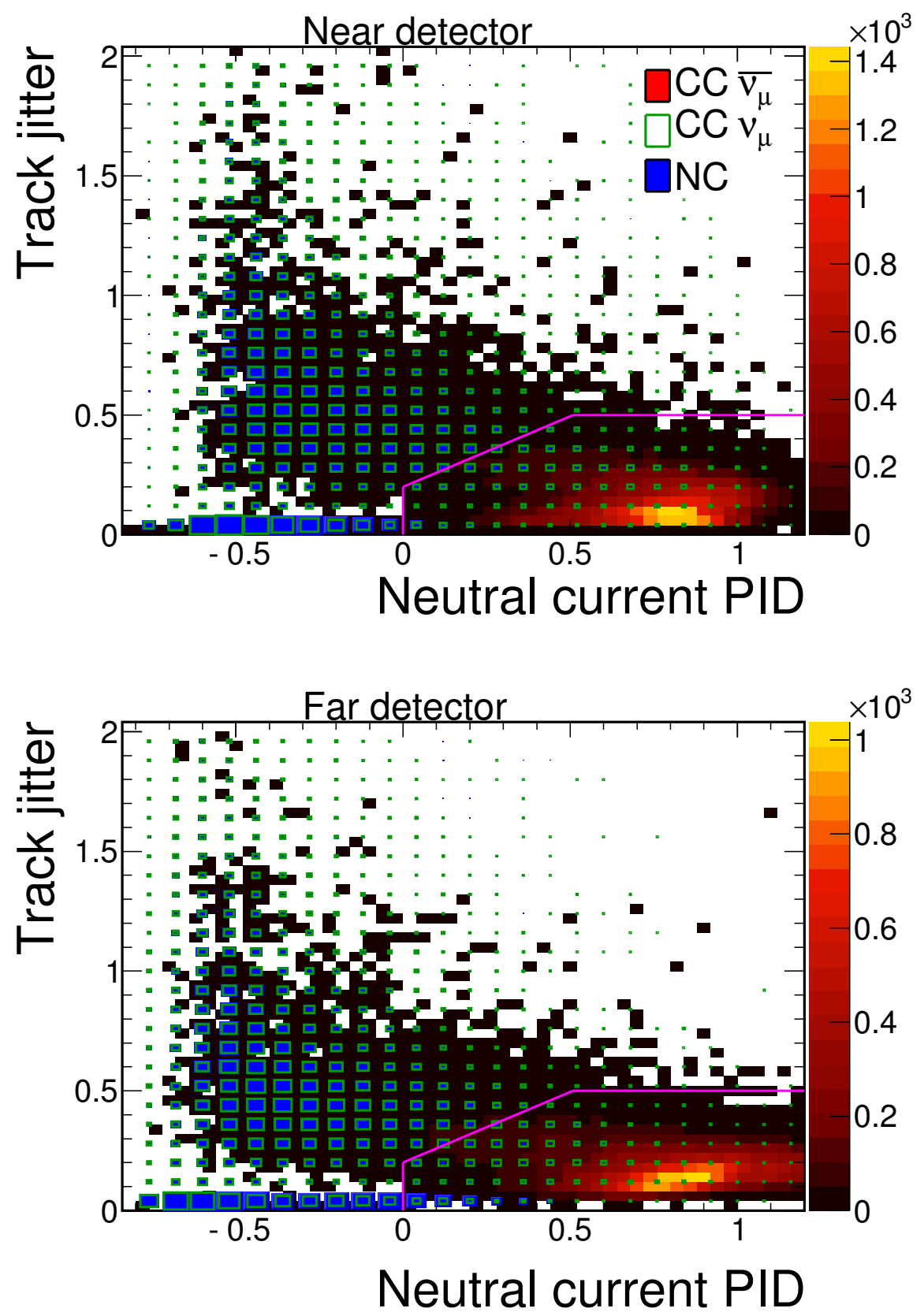

Figure 6.3: Track jitter as a function of the neutral current PID (equation 6.1) for the set of events in figure 6.1, as in figure 6.2, except that here the magenta line shows the cut used: all events above and to the left of the line are rejected. Top: near detector; bottom: far detector. 

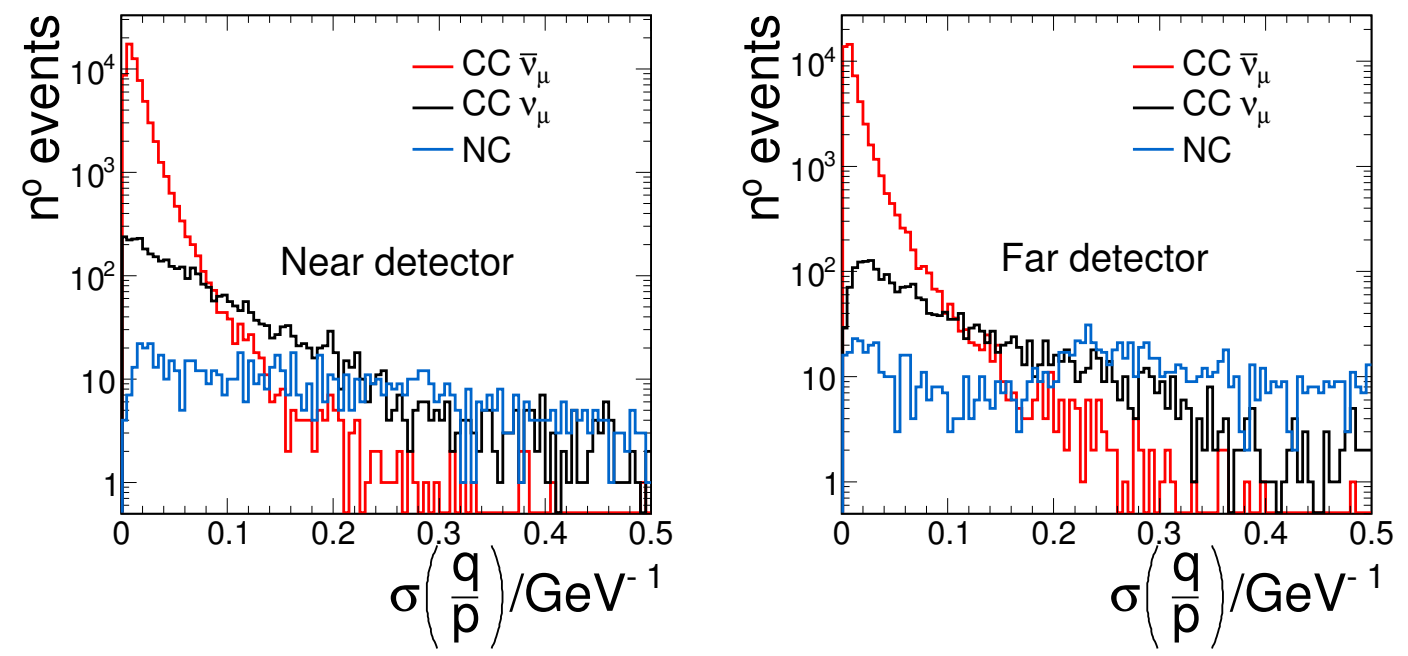

Figure 6.4: Track fit uncertainty $\sigma\left(\frac{q}{p}\right)$ of events passing the preselection, reconstructed charge and majority curvature cuts and neutral current removal, as for figure 5.1. Left: near detector; right: far detector.

The wrong-sign $\mathrm{CC} \nu_{\mu}$ events and any remaining $\mathrm{NC}$ events have a typically higher $\sigma\left(\frac{q}{p}\right)$ than the true $\mathrm{CC} \bar{\nu}_{\mu}$ events. A cut is made, rejecting all events with $\sigma\left(\frac{q}{p}\right)>0.065$. In the near and far detector respectively this rejects $41.1 \%$ and $49.8 \%$ of the remaining wrong-sign $\mathrm{CC} \nu_{\mu}$ events, $1.6 \%$ and $2.1 \%$ of the remaining CC $\bar{\nu}_{\mu}$ events and $78.5 \%$ and $81.8 \%$ of the other remaining (mainly NC) events.

\section{Track Fit Probability}

The reconstruction software returns a $\chi^{2}$ for the fit to the track and a number of degrees of freedom $N_{F}$. This $\chi^{2}$ is calculated from the distance, at each plane, between the best fit track and the actual track hit (the sum runs over every plane in the track). The $\chi^{2}$ and $N_{F}$ can be expressed as a track fit probability $P_{\text {fit }}$. Taking the fit to the detected track to be $H_{0}, P_{\text {fit }}$ is the probability of obtaining a fitted track $t$ with a worse agreement with the detected track than $H_{0}$. Taking a true $\chi^{2}$ distribution $g$, this can be written [58]

$$
P_{\text {fit }}=\int_{t_{\text {observed }}}^{\infty} g\left(t \mid H_{0}\right) \mathrm{d} t
$$



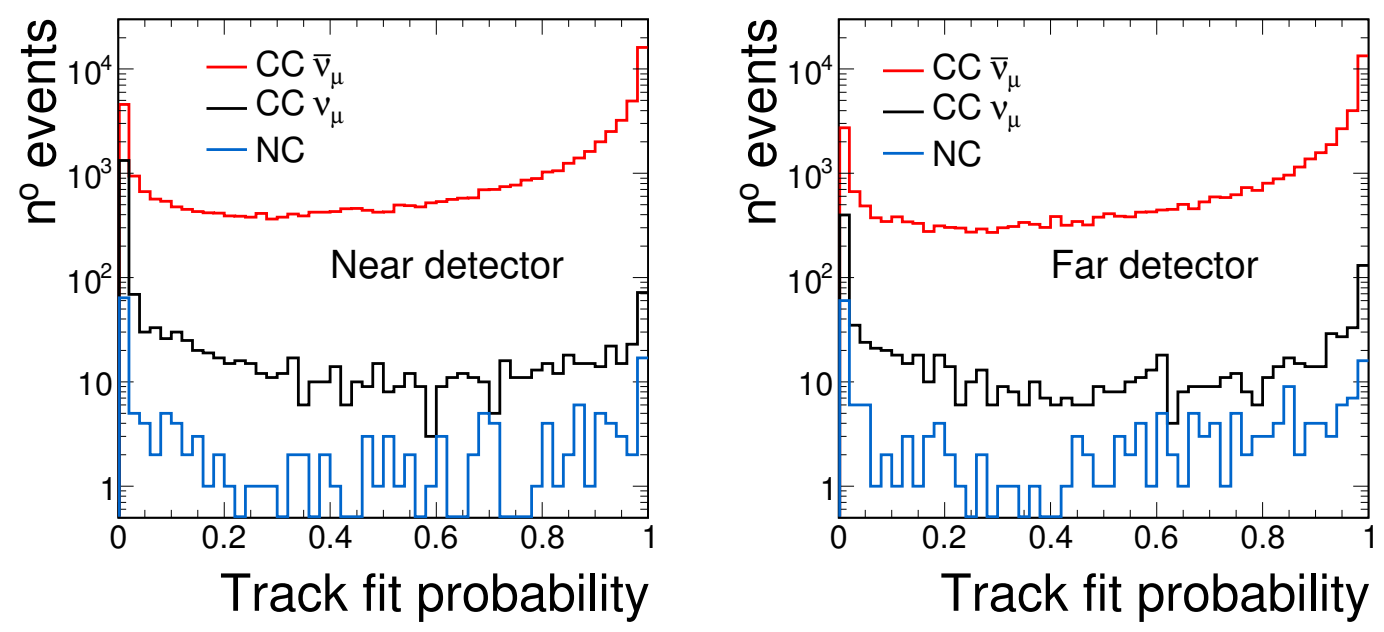

Figure 6.5: Track fit probability $P_{\text {fit }}$ of events passing the preselection, reconstructed charge and majority curvature cuts, neutral current removal and track fit uncertainty cut, as for figure 5.1. Left: near detector; right: far detector.

where higher values of $t$ refer to worse agreement than $H_{0}$.

$P_{\text {fit }}$ for events passing the preceding cuts is shown in figure 6.5. The remaining background of wrong-sign $\mathrm{CC} \nu_{\mu}$ and $\mathrm{NC}$ events peaks sharply at low $P_{\text {fit }}$. All events with $P_{\text {fit }}<0.1$ are rejected. In the near and far detector respectively this rejects $69.2 \%$ and $43.4 \%$ of the remaining wrong-sign $\mathrm{CC} \nu_{\mu}$ events, $12.1 \%$ and $9.7 \%$ of the remaining $\mathrm{CC} \bar{\nu}_{\mu}$ events and $46.3 \%$ and $37.1 \%$ of the other remaining (mainly NC) events.

\section{Track Length}

After making all of the aforementioned cuts, the primary track length (in planes) of the remaining events is shown in figure 6.6.

The majority of the wrong-sign $\mathrm{CC} \nu_{\mu}$ and $\mathrm{NC}$ background events fall at low track lengths. Additionally, confidence in the ability to reconstruct the curvature of a track falls with track length. Therefore all events with tracks shorter than 35 planes are removed. 

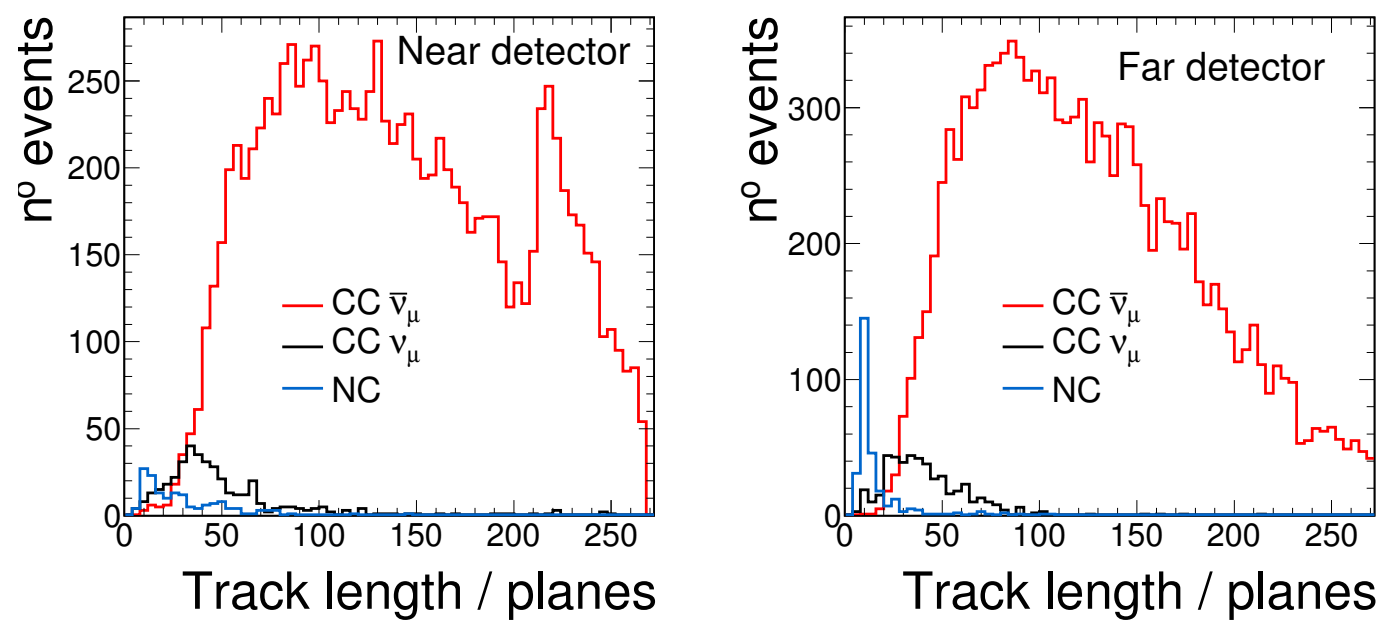

Figure 6.6: The track length of events passing all the preceding selection cuts in the near (left) and far (right) detectors, as for figure 5.1. Left: near detector; right: far detector.

\subsubsection{Overall Selection}

The complete set of selection cuts is summarised in table 6.1. The efficiency and purity of the selection (see equations 5.1 and 5.2) are shown, as a function of energy, in figure 6.7 for the near and far detectors, along with the selected energy spectra.

Below $10 \mathrm{GeV}$ the selection in the near detector has an efficiency of $74.4 \%$ and a purity of $96.5 \%$; in the far detector the efficiency is $70.9 \%$ and the purity $98.8 \%$.

\begin{tabular}{|l|l|r|}
\hline Variable & Reject event if: & $\begin{array}{r}\text { \% preselected } \\
\text { events remaining }\end{array}$ \\
\hline $\mathrm{N}^{\circ}$ tracks & 100 \\
Reconstructed charge & $<0$ & 20.22 \\
Majority curvature & $<0$ & 15.29 \\
Neutral current PID & $<0.0$ & 6.65 \\
Track jitter & $>0.5$ & 6.22 \\
Track jitter & $>0.59 \times(\mathrm{NC}$ PID $)+0.2$ & 5.78 \\
Track fit uncertainty & $>0.065$ & 5.45 \\
Track fit probability & $<0.1$ & 4.74 \\
Track length & $<35$ planes & 4.72 \\
\hline
\end{tabular}

Table 6.1: A summary of the charged current $\bar{\nu}_{\mu}$ selection cuts, showing the per centage of preselected near detector Monte Carlo events remaining after each cut. 

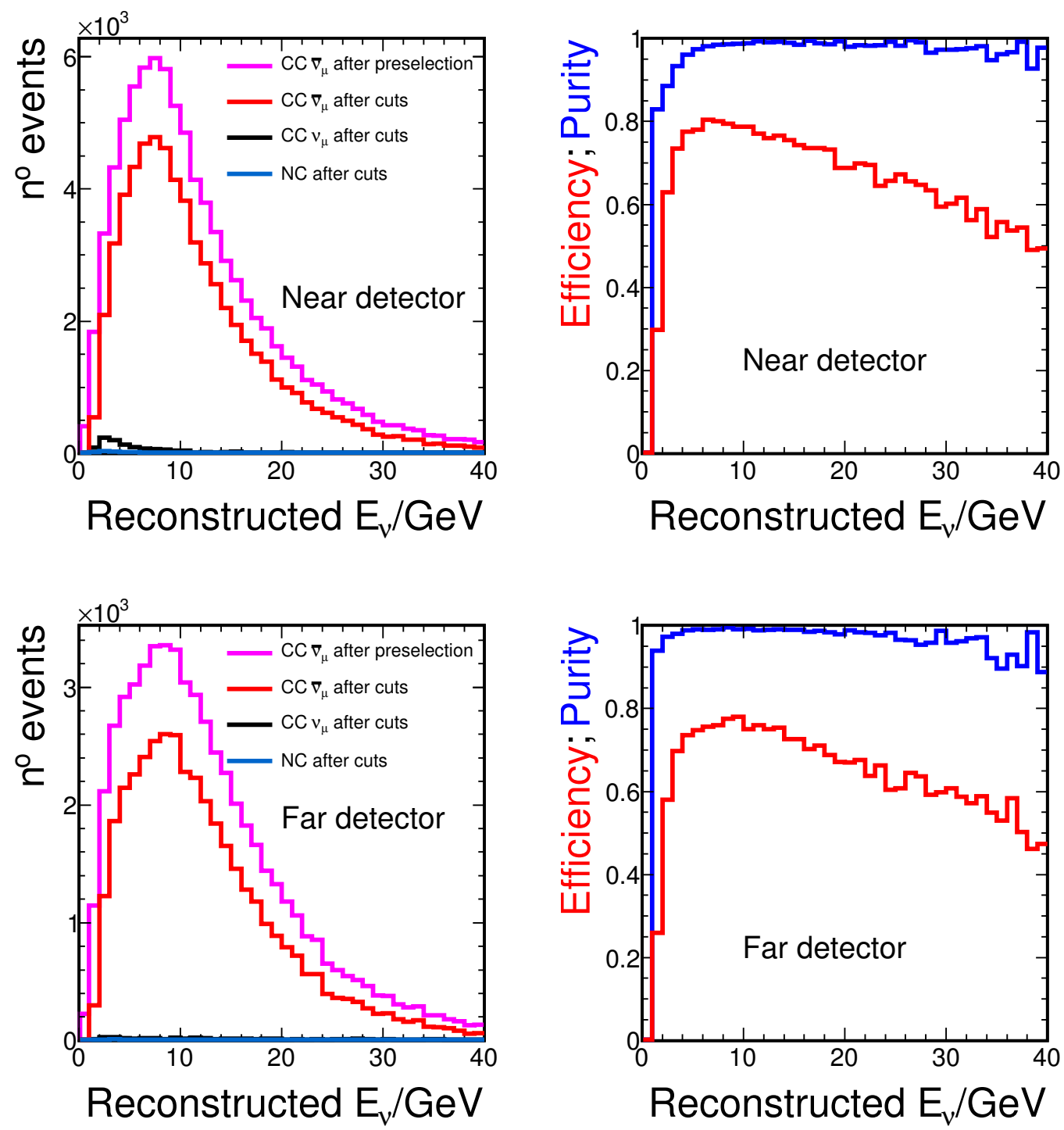

Figure 6.7: Left: reconstructed energy spectra of neutrino interactions passing the complete antineutrino selection (red, black, blue); and the reconstructed energy spectrum of all CC $\bar{\nu}_{\mu}$ interactions which pass the preselection (magenta). Right: the corresponding efficiency (red) and purity (blue) of the CC $\bar{\nu}_{\mu}$ selection, as defined in equations 5.1 and 5.2. Top: near detector. Bottom: far detector. 


\subsection{Agreements Between Distributions in the Data and Simulation}

Comparisons between the distributions of the selection variables in data and Monte Carlo can only be made in the near detector, where the data statistics are high enough to enable such a comparison.

Figure 6.8 shows the comparison for the charge identification variables, $\frac{q}{p}$ and $C_{M}$, before any of the other selection cuts have been made (only the fiducial cut has been made, and the presence of a track in the event required). Figure 6.9 shows these same distributions, after applying all the other selection cuts.

Majority curvature shows an excess in the data near zero, both before and after the cleaning cuts. This region contains tracks for which the direction of curvature is the least certain, for example having similar numbers of track segments which curve in both the positive and negative directions. This suggests a mismodelling in multiple scattering or alignment of the detector planes or scintillator strips: an effect which will work to randomise the directions of the tracks. This is consistent with the distributions for $\frac{q}{p}$, which show an excess of events in the data with $\frac{q}{p} \sim 0$ : these are high reconstructed momentum, that is comparatively straight, tracks. The randomisation produced by the mismodellings may well be acting to wash out the curvature of the magnetic field to some extent, making the tracks in the data appear straighter than those in the Monte Carlo.

The $\frac{q}{p}$ data to Monte Carlo ratio becomes flatter after the cleaning cuts, suggesting these cuts are working to eliminate the tracks most affected by the mismodelling. In the data, after the cleaning cuts, $10.6 \%$ of events have $\frac{q}{p}>0$; in the Monte Carlo the figure is $10.9 \%$. Thus, although the discrepancies in the data to Monte Carlo ratio appear at the $10 \%$ level, few events are migrating past the position of the cut which would be the concern for the overall selection. Similarly for majority curvature, after cleaning $12.0 \%$ of events have $C_{M}>0$ in the data 

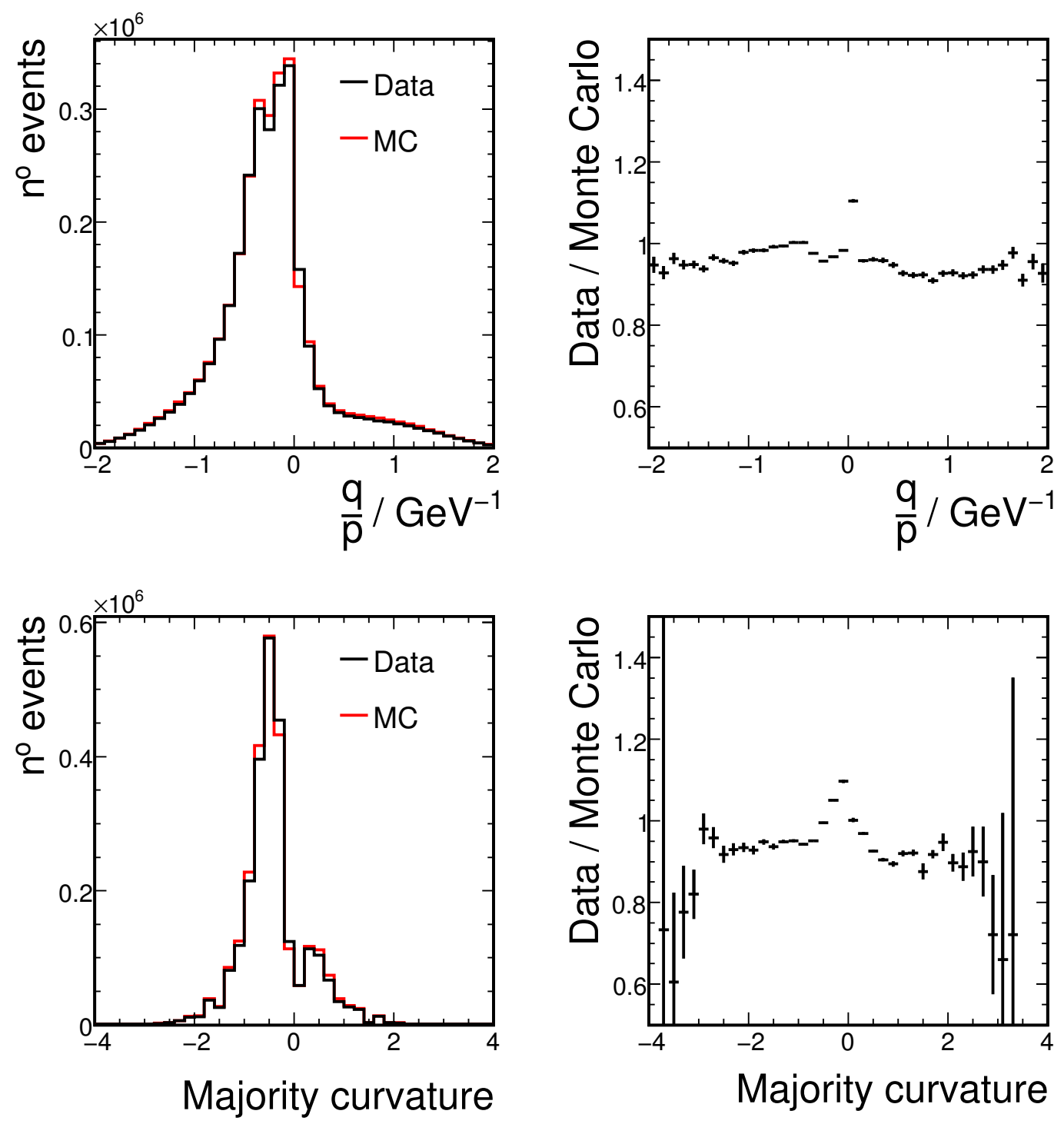

Figure 6.8: Left: the near detector data (black) and Monte Carlo (red) distributions of $\frac{q}{p}$ (top) and $C_{M}$ (bottom) after making only the preselection cuts. Right: the ratio of data to Monte Carlo. The histograms are normalised by exposure (to the same number of protons on target). 

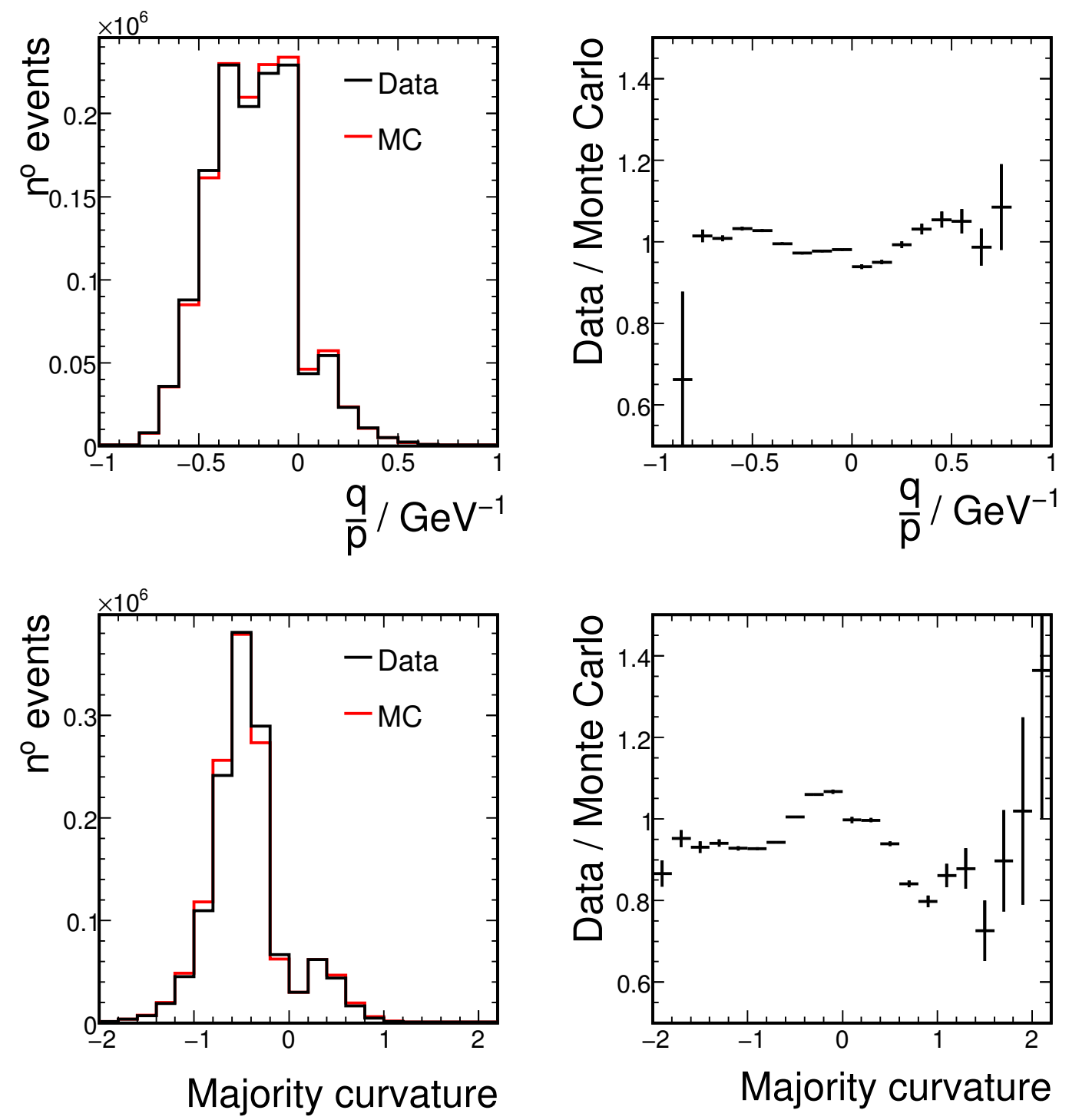

Figure 6.9: The near detector data and Monte Carlo distributions of $\frac{q}{p}$ (top) and $C_{M}$ (bottom) after making all other selection cuts, displayed as in figure 6.8. 

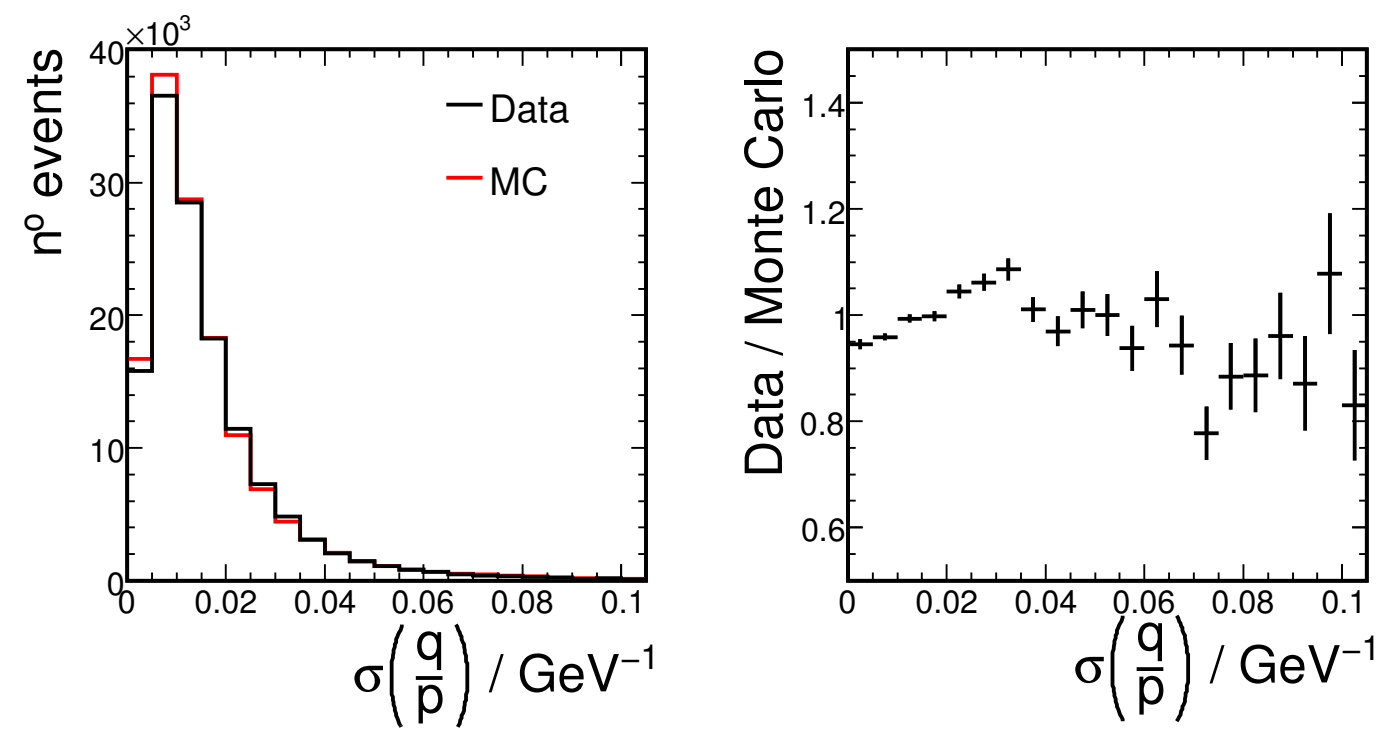

Figure 6.10: The near detector data and Monte Carlo distributions of $\sigma\left(\frac{q}{p}\right)$ after making all other selection cuts, displayed as in figure 6.8.

and $12.4 \%$ in the Monte Carlo, again suggesting minimal migration past where the cut is placed.

The data and Monte Carlo distributions for $\sigma\left(\frac{q}{p}\right)$ are compared in figure 6.10. The deficit in data at low $\sigma\left(\frac{q}{p}\right)$, with an increasing data excess as $\sigma\left(\frac{q}{p}\right)$ increases towards $\sim 0.03$, again suggests a mismodelling causing the tracks in data to have less certain curvature than in the Monte Carlo (again, perhaps multiple scattering or detector alignment). However, the cut at $\sigma\left(\frac{q}{p}\right)=0.065$ is in a region with low statistics, which is furthermore a region where the data to Monte Carlo agreement is good, giving confidence that the cut is not biased by the mismodellings. The theory that mismodellings are causing greater curvature randomisation in the data is further supported by the track jitter variable, as shown in figure 6.11 after performing all cuts except those on $P_{D}$ and $J$. An excess of high randomization $(J \sim 0.4)$ events is seen in the data with a deficit of low randomization events $(J \sim 0)$. The $50 \%$ deficit in the lowest bin of the track jitter distribution is not a concern, as figure 6.3 shows these events to be primarily neutral current events which are removed by the $P_{D}$ cut. 

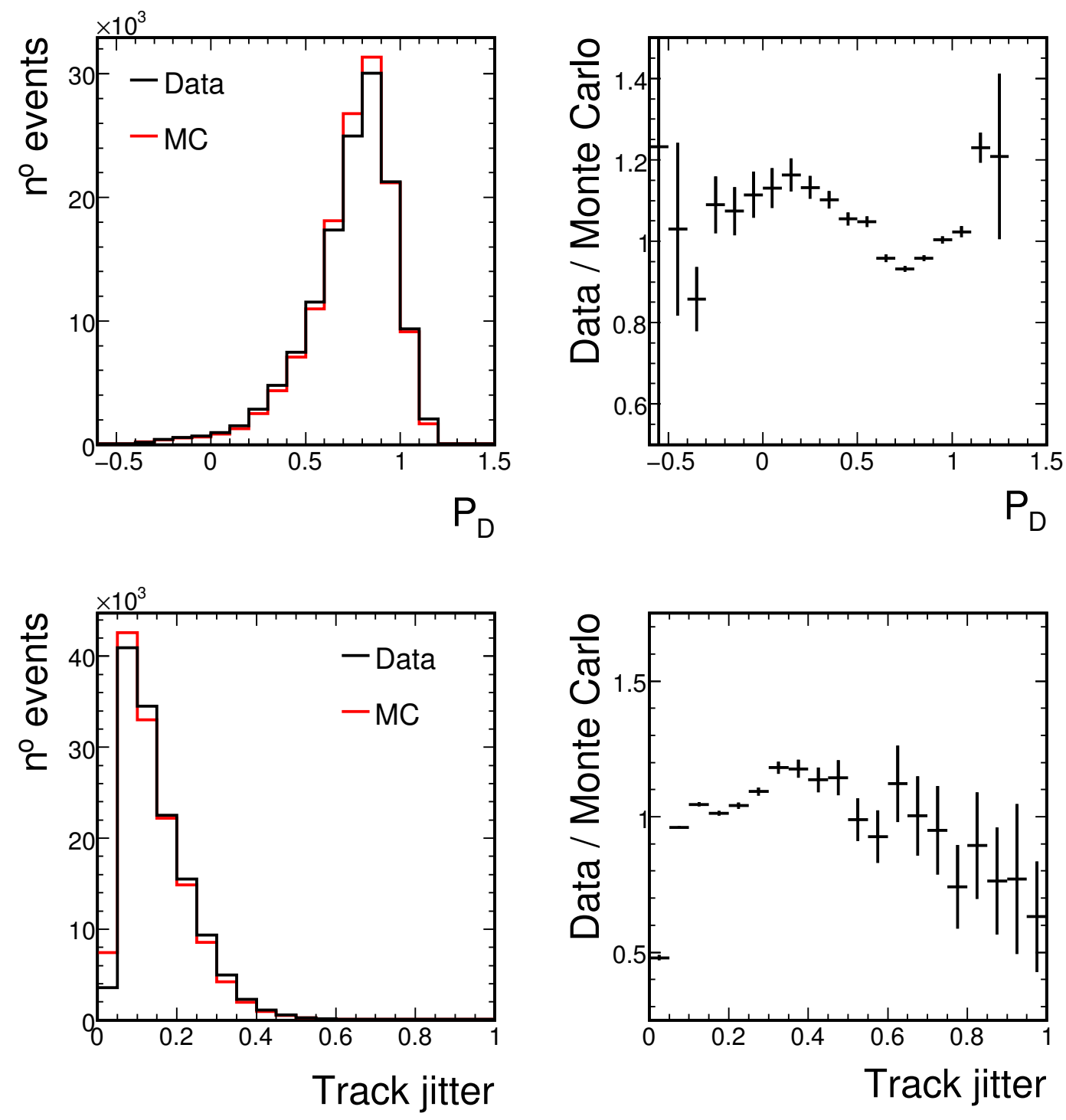

Figure 6.11: The near detector data and Monte Carlo distributions of $P_{D}$ (top) and $J$ (bottom) after making all other selection cuts, displayed as in figure 6.8. 

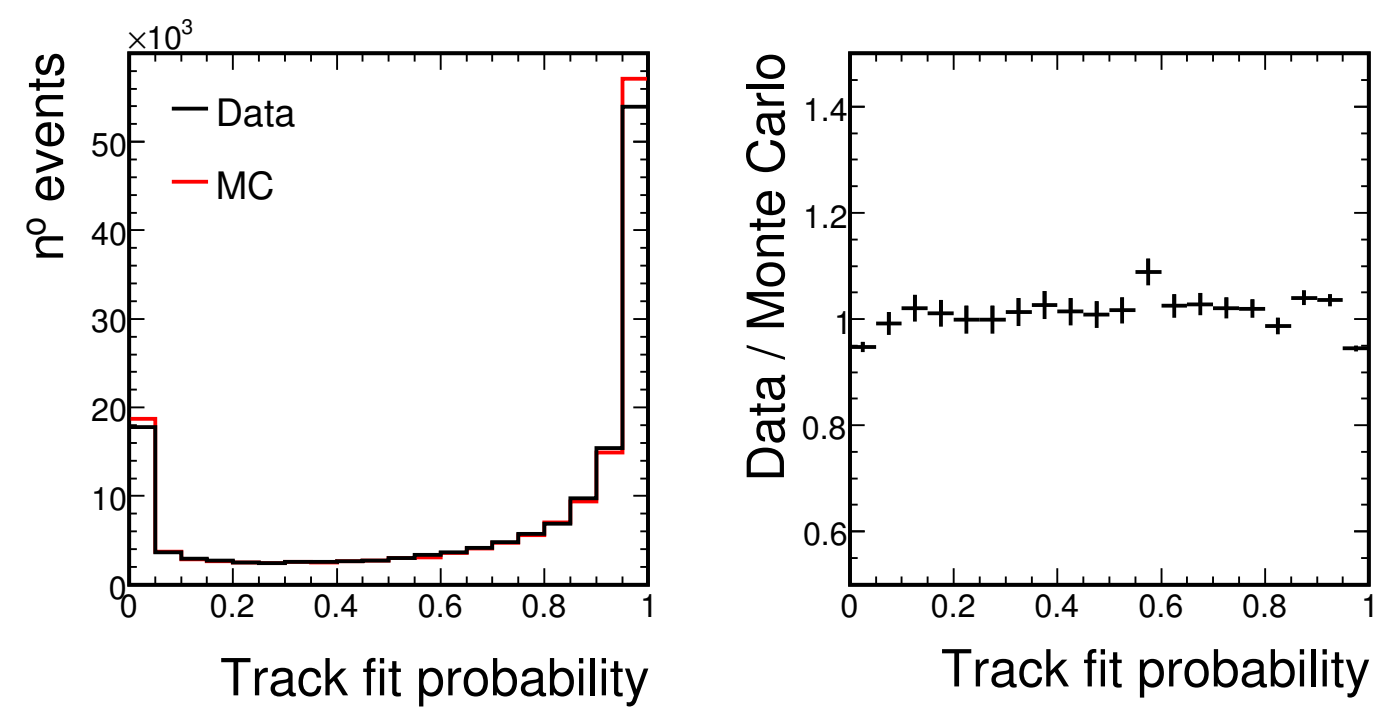

Figure 6.12: The near detector data and Monte Carlo distributions of $P_{\text {fit }}$ after making all other selection cuts, displayed as in figure 6.8.

The data to Monte Carlo comparison of $P_{D}$, also shown in figure 6.11, is harder to interpret in terms of physical effects as the variable is a mathematical combination of variables to form a distribution not directly connected with aspects of the detector. However, the general pattern of a data excess in the background region (low $P_{D}$ ) and a corresponding deficit in the signal region (high $P_{D}$ ) matches that seen in the other variables. Again, the cuts on $P_{D}$ and $J$ are performed primarily in regions of low statistics $\left(P_{D}=0\right.$ and $J=0.5$ being the hard cuts on each variable). Figure 6.3 shows the two-dimensional part of the cut to be acting away from where the majority of the signal and background statistics fall.

The data and Monte Carlo distributions of track fit probability (figure 6.12) show the agreement for this variable to be very good, giving confidence in the cut. The corresponding distributions for track length are shown in figure 6.13, and show the cut to acting in a region of low statistics, limiting the possible effects of any mismodelling.

Figure 6.14 shows the reconstructed energy distribution in the near detector after the full selection for the data and Monte Carlo. The disagreements of 

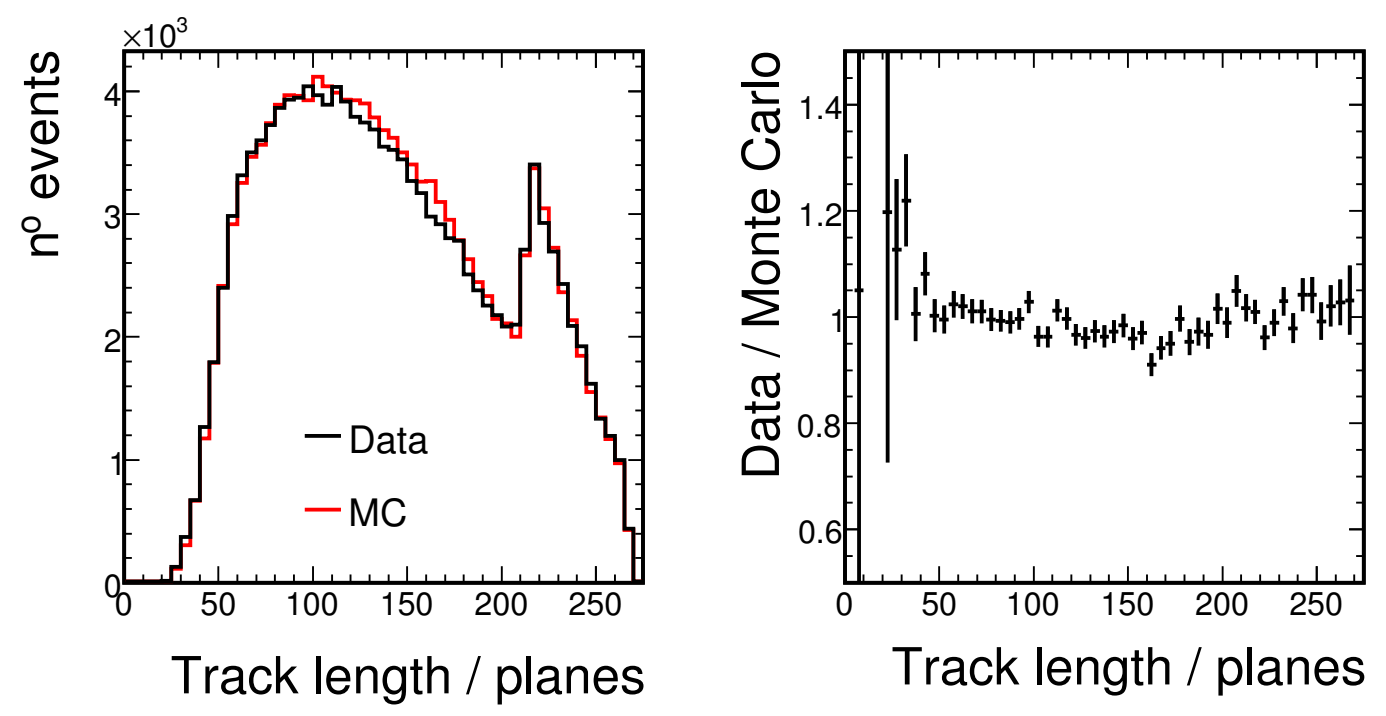

Figure 6.13: The near detector data and Monte Carlo distributions of track length after making all other selection cuts, displayed as in figure 6.8.
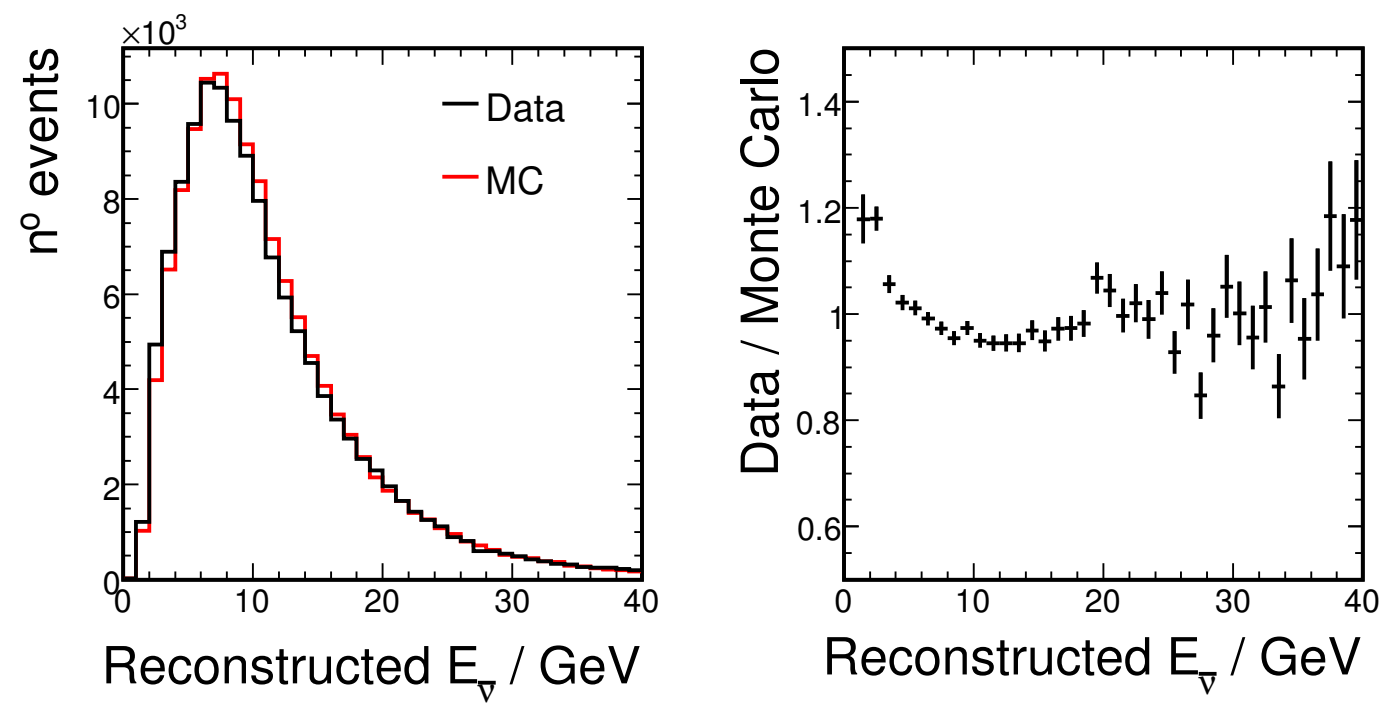

Figure 6.14: The near detector data and Monte Carlo distributions of reconstructed neutrino energy after making all selection cuts, displayed as in figure 6.8. 
$<20 \%(<5 \%$ across most the spectrum) could come from the mismodellings discussed above, or alternatively from mismodellings in the beam spectrum, $\bar{\nu}_{\mu}$ cross sections, or detector effects such as calibration. The extrapolation procedure described in chapter 7 aims to minimise the effect on the analysis of uncertainties which affect both detectors (such as beam or cross section uncertainties) by using the disagreement between these data and Monte Carlo energy spectra to appropriately correct the far detector Monte Carlo. Uncertainties in background estimation are more of a concern (particularly if they affect the two detectors differently). The neutral current background is negligible (see figure 6.7), so uncertainties here should affect the analysis minimally. The wrong-sign charged current $\nu_{\mu}$ background is larger, and falls in the most likely signal region (although it is still a low level background). In chapter 8 uncertainties on these backgrounds, along with all other possible sources of uncertainty, are estimated and the effects on the analyses calculated.

\subsection{Neutrino Event Selection}

The antineutrino analyses presented in the rest of this thesis also require a sample of neutrinos, to enable the neutrino background in the far detector antineutrino sample to be best estimated. The neutrino selection used [174] is applied to any events with a primary track having $\frac{q}{p}<0$ and aims primarily to minimise the neutral current background. It uses four variables: the number of scintillator planes in the track, the mean pulse height per plane in the track, a measure of the width of the reconstructed track (its transverse profile in the detector), and a measure of the amount of fluctuation in the response per scintillator strip in the track. The variables are combined in a $k$-nearest-neighbour approach: each data event is put into a four-dimensional space (along with all Monte Carlo events), the axes of this space being the four variables described above. Looking at the $k$ 

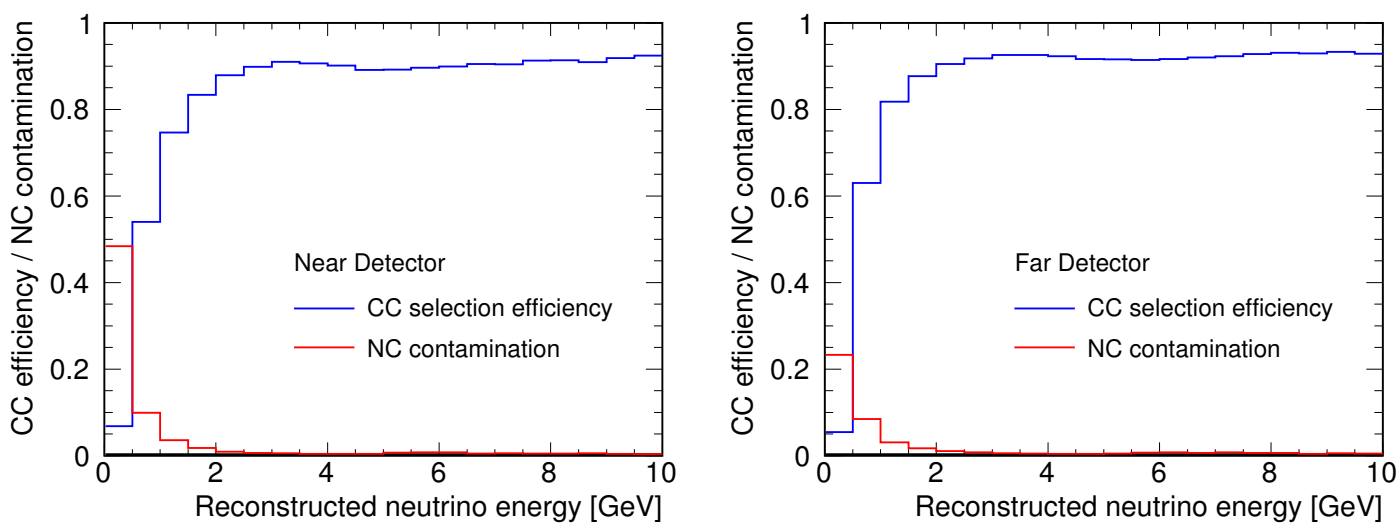

Figure 6.15: The charged current $\nu_{\mu}$ efficiency (blue) and contamination from neutral current events (red) achieved by the $k$-nearest-neighbour selection technique. (Left: near detector. Right: far detector.)

Monte Carlo events nearest to the data event in that space gives a measure of how likely that event is to be signal or background. The efficiency and purity achieved by this technique is shown in figure 6.15.

The reason this new identification technique is used rather than that discussed in section 6.3 .3 is simply that this is a more modern development. The variable of 6.3.3 is perfectly adequate for the $\bar{\nu}_{\mu}$ selection; future $\bar{\nu}_{\mu}$ analyses may choose to investigate the use of this new $k$-nearest-neighbour technique.

\subsection{Summary}

A high purity charged current $\bar{\nu}_{\mu}$ event selection has been developed, the same selection being used in both MINOS detectors. Below $10 \mathrm{GeV}$ the efficiencies and purities are, respectively, $74.4 \%$ and $96.5 \%$ in the near detector and $70.9 \%$ and $98.8 \%$ in the far (the primary background being misidentified charged current $\nu_{\mu}$ events). Comparison of the variables used for selection in the near detector data and Monte Carlo suggest possible mismodellings of muon curvature (perhaps in multiple scattering or detector plane alignment), but these are not expected to significantly bias the analysis. The final disagreements between the selected data 
and Monte Carlo energy spectra will be corrected for through the extrapolation procedure described in chapter 7 . 


\section{Chapter 7}

\section{Antineutrino Extrapolation}

\subsection{Inter-Detector Flux Differences}

The two-detector philosophy of the MINOS experiment is intended to allow the cancellation of systematic uncertainties. A measurement of the neutrino spectrum at the near detector should enable a prediction of the spectrum at the far detector in the case of no oscillations. This reduces the effect of systematic uncertainties which would affect both detectors in the same way: for example uncertainties in neutrino cross sections, neutrino flux from the beam, hadronic shower modelling and track energy measurements from range.

This cancellation only works exactly in the case that the neutrino flux is the same (barring scale factors) at both detectors. However for MINOS this is not exactly true. Figure 7.1 shows neutrino parents decaying in the NuMI decay pipe. The far detector, $735 \mathrm{~km}$ distant, subtends a very small solid angle to the parent. Only one decay direction of the parent can produce a neutrino that will pass through the far detector. Thus the corresponding neutrino energy is uniquely constrained. However, the near detector subtends a much larger solid angle: a range of decay angles of the parent produce a neutrino that will pass through the detector, corresponding to a range of neutrino energies. Furthermore, parents 


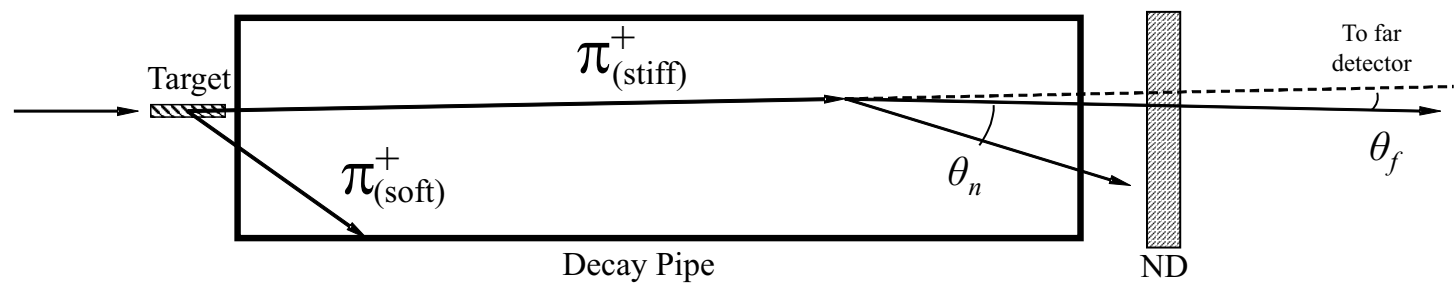

Figure 7.1: A diagram (not to scale) of neutrino parents in the NuMI decay pipe, illustrating the different solid angles subtended by the near and far detectors at the parent decay point.
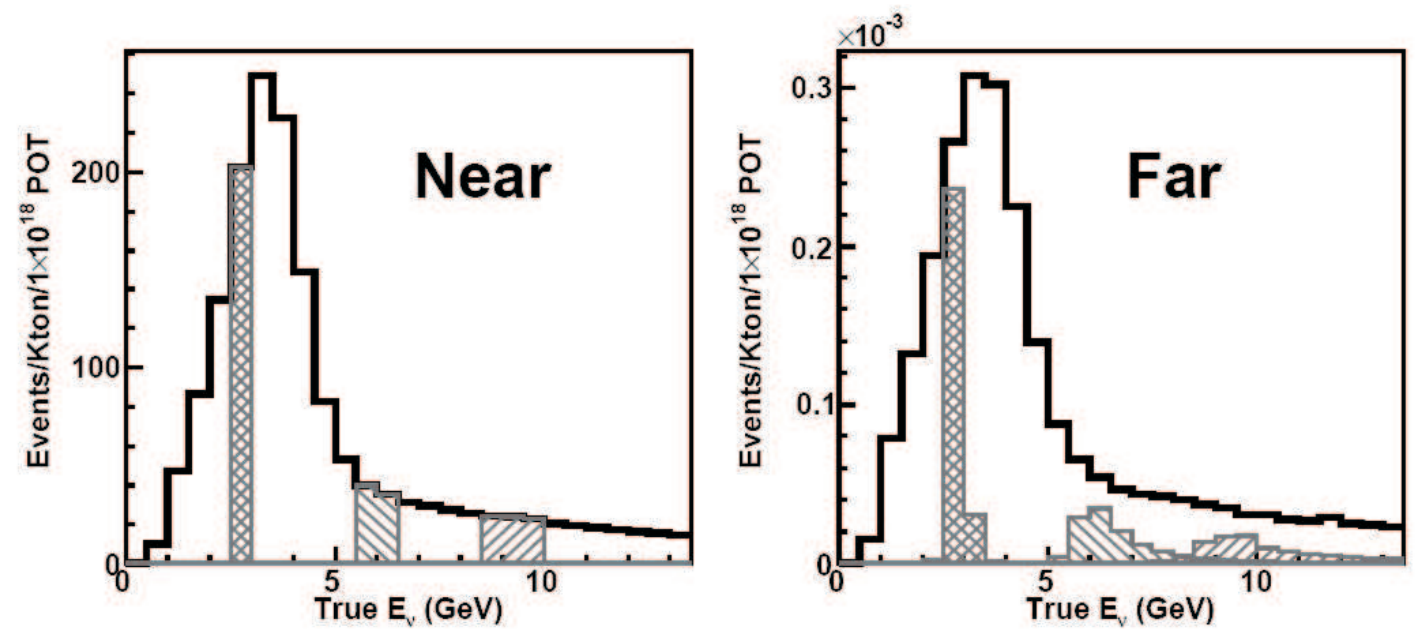

Figure 7.2: Simulated true neutrino energy spectra in the near (left) and far (right) detectors. Parents responsible for producing neutrinos in the hatched near detector energy bins produce a different neutrino energy distribution in the far detector, shown by the corresponding hatching. (Figure taken from [159].)

decay along the full length of the decay pipe, the solid angle subtended by the near detector differing considerably along this length. Therefore the near to far spectral difference cannot be removed simply by making a solid angle cut at the near detector.

Figure 7.2 illustrates the effect of parent decay kinematics on the near and far detector energy spectra. It highlights how parents responsible for certain near detector neutrino energies produce a different far detector neutrino energy distribution. The effect is most pronounced for higher energy parents (and therefore higher energy neutrinos) which typically travel further along the decay pipe before decaying: the neutrinos are then produced closer to the near detector, allowing 
a wider range of contributing decay angles. (This effect outweighs the increased Lorentz boost of the higher energy parents, which narrows the range of decay angles in the laboratory frame.) The effect primarily lowers the typical energy of a neutrino at the near detector in comparison to the far as the parents are travelling predominantly towards the far detector. The neutrino has the highest energy in the laboratory frame when the parent decays directly forward: the far detector therefore preferentially selects higher-energy neutrinos. The overall effect is to take neutrinos from above the peak in the far detector energy spectrum and move them downwards in energy in the near detector. This moves them into the peak, causing the near detector spectrum to appear more peaked than the far detector spectrum.

\subsection{Extrapolation Methods}

To overcome these inter-detector flux differences, a number of methods have been developed to use the near detector data to predict a far detector spectrum. These methods additionally allow far detector spectra to be predicted in the case of neutrino oscillations: a non-trivial process as the oscillations must be applied as a function of true neutrino energy; the near detector data only supplies the reconstructed neutrino energy spectrum.

The various extrapolation methods, four of which are detailed in [159], fall into two general categories. One category compares the near detector data to Monte Carlo, to provide a set of parameters that can be used to transform the existing far detector Monte Carlo. The other category provides a set of transformations to the near detector data to turn it into a far detector prediction. In this thesis a method of the second category, the beam matrix method, is developed for an antineutrino analysis. This method was first proposed by M. Szelper and A. Para [175]. 


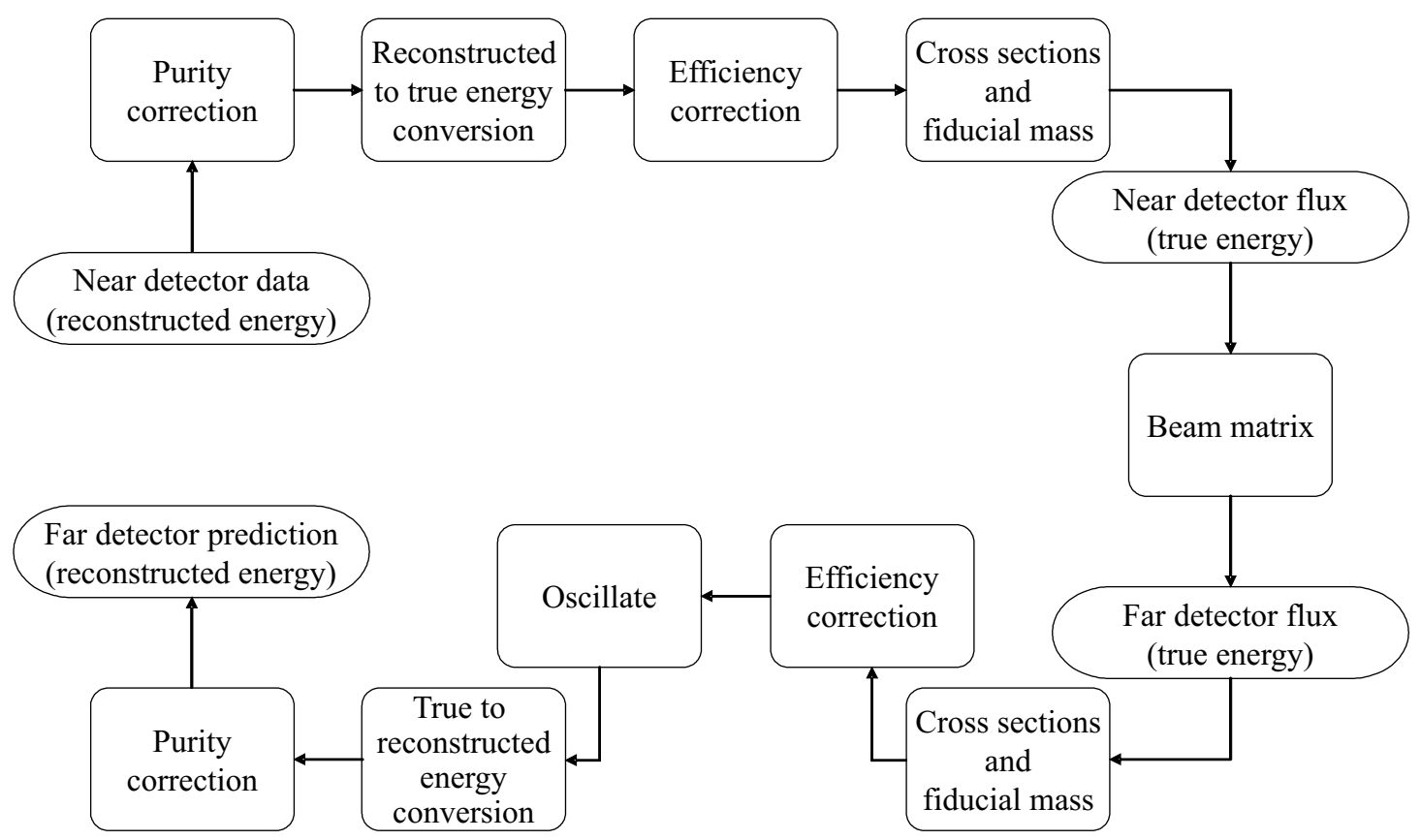

Figure 7.3: The steps involved in the beam matrix extrapolation of the MINOS neutrino oscillation analysis $[158,159]$.

\subsection{Beam Matrix Extrapolation}

The beam matrix method was the primary extrapolation method used for the previously published MINOS neutrino oscillation analysis [158, 159]. A set of transformations are applied to the near detector data spectrum to convert it first into a near detector flux, then to a far detector flux, finally to a far detector reconstructed energy spectrum. These transformations are effected through the multiplication of the spectrum by certain matrices (some of which are diagonal). The procedure as used for the aforementioned neutrino analysis is shown in figure 7.3 .

The near detector spectrum is firstly corrected to remove impurities (misidentified neutral current events, see section 3.8.1). The application of a nondiagonal matrix then transforms the reconstructed neutrino energy spectrum into a spectrum of true neutrino energies. An efficiency correction is applied to take out the effect of selection cuts and reconstruction inefficiencies, producing the 
true energy spectrum of all neutrinos which interacted in the fiducial volume. The dividing out of neutrino cross sections and fiducial detector mass follows to produce the neutrino flux passing through the near detector.

A beam matrix is calculated from a Monte Carlo simulation of the neutrino beam, which acts on a near detector neutrino flux to produce a far detector flux. To produce this matrix, the flux of neutrino parents is split (as detailed below) into representative sets of parents, one set for each bin in near detector neutrino energy. The matrix works by scaling each set to be consistent with the measured near detector neutrino flux. The contribution of each scaled set of parents to the far detector neutrino flux is calculated, and these contributions summed to give the total far detector neutrino flux prediction.

At the far detector, the transformations described for the near detector are applied in reverse order. The cross sections and fiducial detector mass are multiplied back in to produce the spectrum of all neutrinos interacting in the fiducial volume, followed by an efficiency correction to reduce that spectrum to that of the neutrinos remaining after event reconstruction and selection cuts. The effect of oscillations is now applied to the energy spectrum (as a function of true neutrino energy). A non-diagonal matrix converts this into a reconstructed energy spectrum, followed by the addition (this time additively) of neutral current impurities. The result is a prediction of the measured far detector energy spectrum.

In this thesis, the procedure is adapted for the analysis of antineutrinos, as shown in figure 7.4. As the $\nu_{\mu}$ and $\bar{\nu}_{\mu}$ spectra are being separated to allow the potential of different oscillation parameters in the $\mathcal{C P} \mathcal{T}$ violation analysis, they must be individually extrapolated. As shown in chapter 6, the primary background for a charged current $\bar{\nu}_{\mu}$ event selection consists of charged current $\nu_{\mu}$ events. This background must be correctly predicted, and oscillated according to the $\nu_{\mu}$ oscillation parameters. The implementation in this thesis thus furthermore differs from that published in $[158,159]$ in the way impurities are added at the 


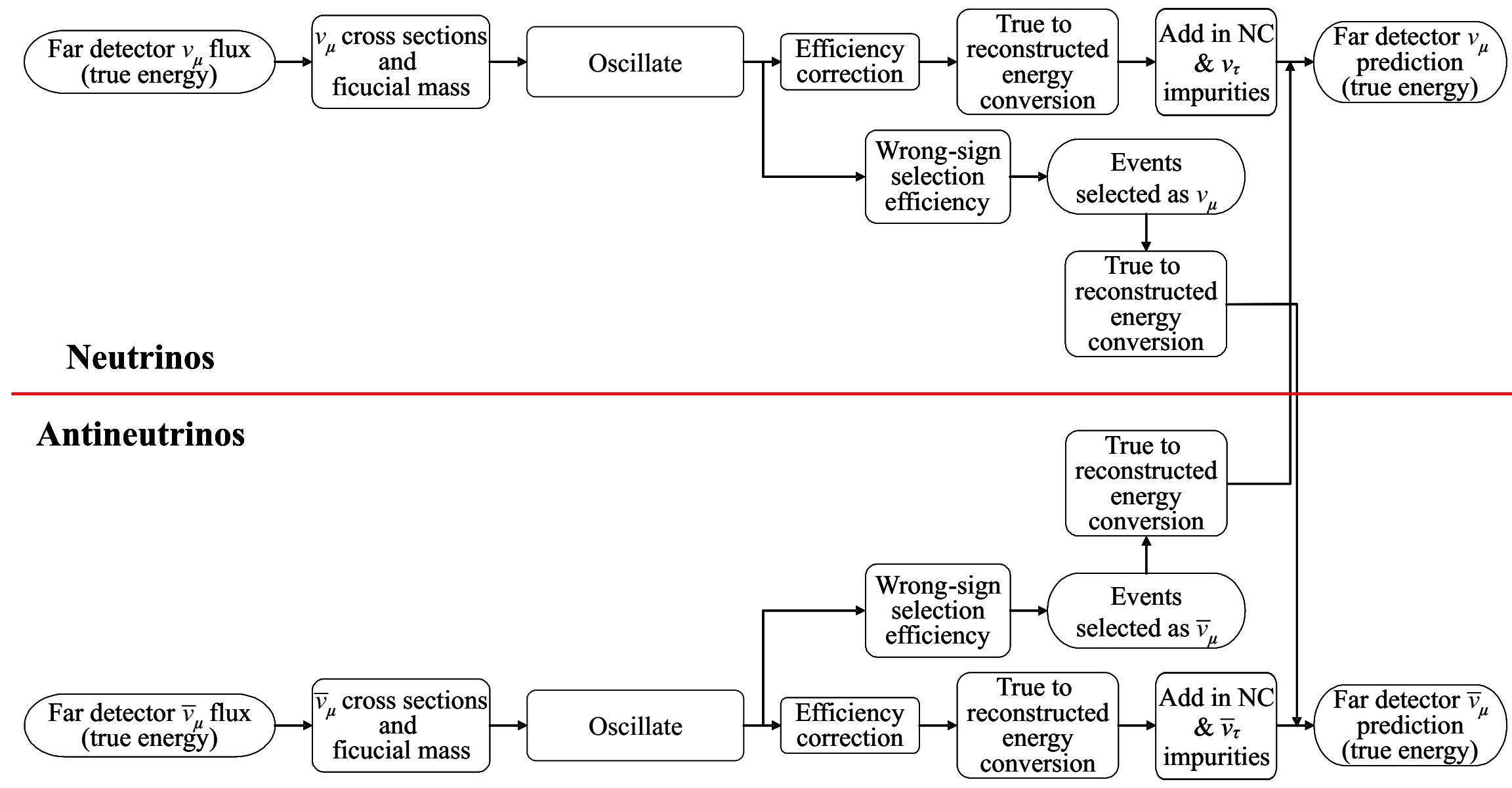

Figure 7.4: The steps involved in the beam matrix extrapolation developed for the analysis of antineutrinos in this thesis. The flowchart begins at the far detector flux calculated using the process illustrated in figure 7.3. 
far detector. The $\nu_{\mu}$ and $\bar{\nu}_{\mu}$ spectra are extrapolated individually, and the $\nu_{\mu}$ prediction used to calculate the charged current background to the far detector $\bar{\nu}_{\mu}$ spectrum, and vice-versa.

This procedure, by design, works with binned energy spectra. The binning chosen is $250 \mathrm{MeV}$ (with a single bin from 0 to $500 \mathrm{MeV}$ ). The procedure has been repeated with alternative binnings, confirming that changing the binning has little effect on the resultant prediction.

The various symbols used in the following description are summarised at the end of the chapter in table 7.1 .

\subsubsection{Near Detector Purity Correction}

The first correction removes impurities from the measured near detector neutrino energy spectrum. A histogram of purity as a function of reconstructed neutrino energy, $P_{i}^{\mathrm{N}}$, is calculated from Monte Carlo, where $i$ is an integer labelling the bin of the histogram and the superscript $\mathrm{N}$ shows that this histogram relates to the near detector (as it will for all subsequent variables in this chapter). This histogram is calculated by applying the relevant selection to the Monte Carlo:

$$
P_{i}^{\mathrm{N}}=\frac{\left(\mathrm{N}^{\mathrm{o}} \text { signal events selected }\right)_{i}}{\left(\text { Total } \mathrm{n}^{\mathrm{o}} \text { events selected }\right)_{i}}
$$

where signal events will refer to either charged current $\nu_{\mu}$ or $\bar{\nu}_{\mu}$ events, whichever are to be extrapolated. This histogram is applied to the measured near detector reconstructed neutrino energy spectrum, $R_{i}^{\mathrm{N}}$, to produce the reconstructed energy spectrum with impurities removed:

$$
R_{i}^{\mathrm{N}, \mathrm{P}}=R_{i}^{\mathrm{N}} P_{i}^{\mathrm{N}} \quad \forall i
$$

where, as throughout this chapter, a repeated index does not imply a summation. 


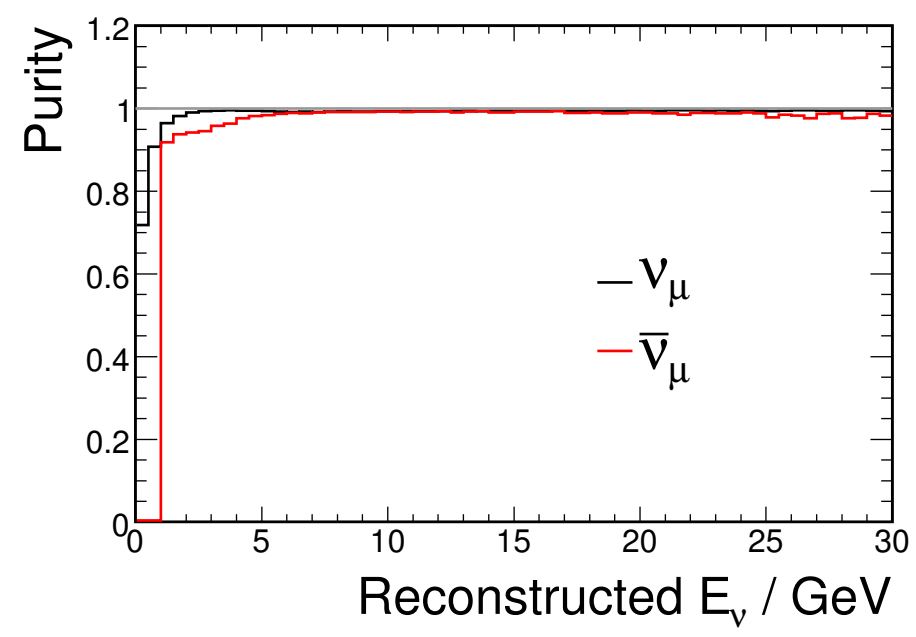

Figure 7.5: The purity correction applied in the near detector.

The purity correction histograms for the charged current $\nu_{\mu}$ and $\bar{\nu}_{\mu}$ selections detailed in chapter 6 are shown in figure 7.5.

\subsubsection{Near Detector Reconstructed to True Energy Matrix}

A matrix $\mathcal{M}_{i j}^{\mathrm{N}}$ is formed to act on the now pure reconstructed energy near detector neutrino spectrum, to obtain the spectrum as a function of true neutrino energy. From the near detector Monte Carlo, every selected signal event for the relevant extrapolation is filled into a two-dimensional histogram $M_{i j}^{\mathrm{N}}$, one axis $(i)$ being the reconstructed neutrino energy, the other $(j)$ the true neutrino energy. (Selected impurities are not put into this histogram as the matrix will be acting on a pure spectrum.)

The histogram (figure 7.6) is normalised to become the matrix by applying a single scale factor, $N_{i}^{\mathrm{N}}$, to each bin in a column $i$ of reconstructed energy such that the sum of bins in that column are unity:

$$
N_{i}^{\mathrm{N}}=\sum_{j} M_{i j}^{\mathrm{N}} \quad \forall i .
$$



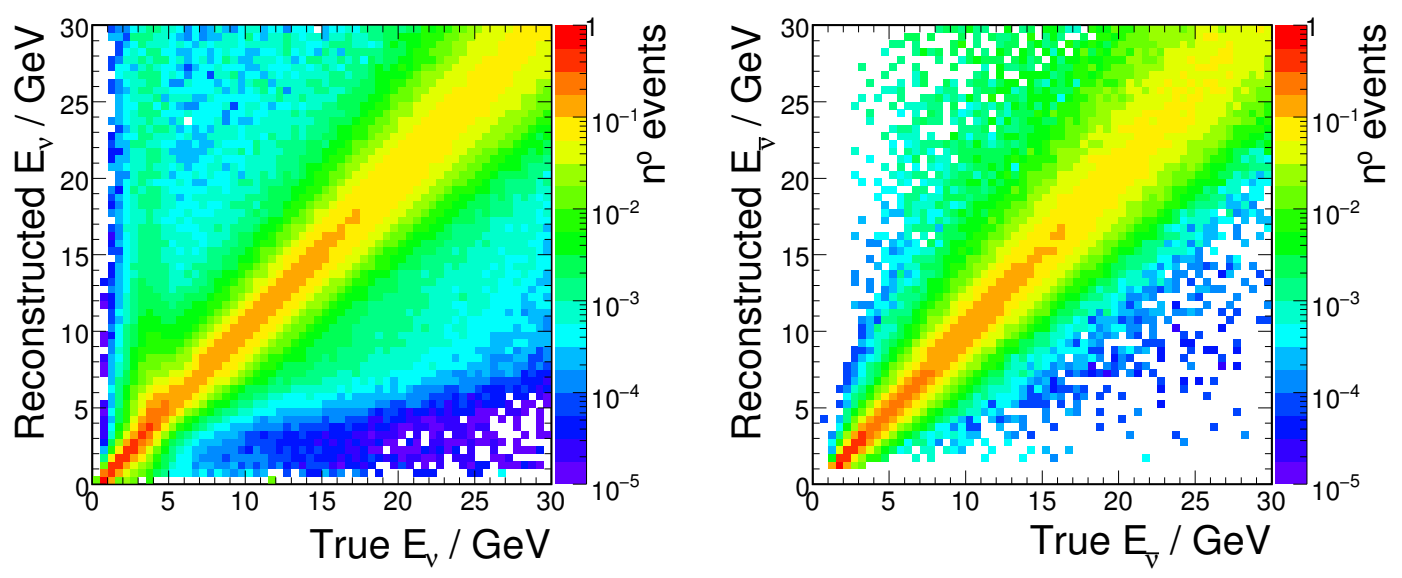

Figure 7.6: The reconstructed to true energy conversion matrices applied at the near detector, for neutrinos (left) and antineutrinos (right).

Then

$$
\mathcal{M}_{i j}^{\mathrm{N}}=\frac{M_{i j}^{\mathrm{N}}}{N_{i}^{\mathrm{N}}} \quad \forall i, j .
$$

Each bin $i$ of the now pure reconstructed energy spectrum $R_{i}^{\mathrm{N}, \mathrm{P}}$ (the superscript $\mathrm{P}$ signifying this distribution is now 'pure') is multiplied by each bin in the column of the matrix corresponding to the same reconstructed energy. The result, $\mathcal{M}_{i j}^{\mathrm{N}} R_{i}^{\mathrm{N}, \mathrm{P}}$, is the contribution of that reconstructed energy bin to the bin $j$ of the selected true energy spectrum $T_{j}^{\mathrm{N}, \mathrm{S}}$ (the superscript $\mathrm{S}$ signifying a 'selected' distribution). This is repeated for every $i$ and the results added into the true spectrum:

$$
T_{j}^{\mathrm{N}, \mathrm{S}}=\sum_{i} \mathcal{M}_{i j}^{\mathrm{N}} R_{i}^{\mathrm{N}, \mathrm{P}} \quad \forall j
$$

The normalisation of $\mathcal{M}_{i j}^{\mathrm{N}}$ ensures that one neutrino in the reconstructed energy spectrum produces one neutrino in the true energy spectrum. 


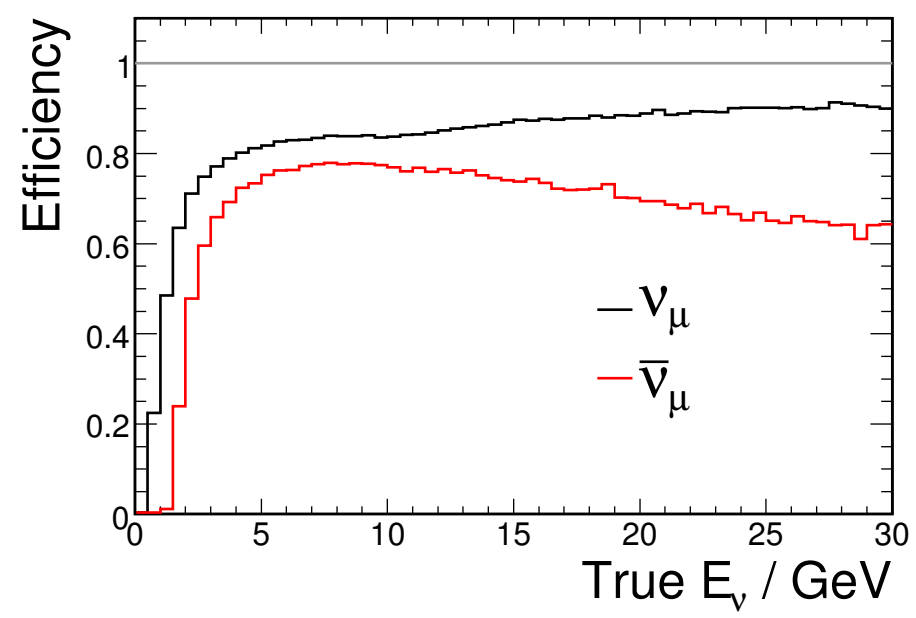

Figure 7.7: The near detector efficiency correction.

\subsubsection{Near Detector Efficiency Correction}

A histogram of efficiency as a function of true neutrino energy for the selection in question, $E_{i}^{\mathrm{N}}$, is filled from Monte Carlo, for each true energy bin $i$ :

$$
E_{i}^{\mathrm{N}}=\frac{\left(\mathrm{N}^{\mathrm{o}} \text { signal events selected }\right)_{i}}{\left(\text { Total } \mathrm{n}^{\mathrm{o}} \text { signal interactions in fiducial volume }\right)_{i}}
$$

This histogram (figure 7.7) is used to correct the selected true energy spec$\operatorname{trum} T_{j}^{\mathrm{N}, \mathrm{S}}$ for selection efficiencies, to give the true energy spectrum of all signal neutrinos interacting in the detector fiducial volume:

$$
T_{i}^{\mathrm{N}}=\frac{T_{i}^{\mathrm{N}, \mathrm{S}}}{E_{i}^{\mathrm{N}}} \quad \forall i
$$

\subsubsection{Calculating the Near Detector Flux}

To obtain the neutrino flux through the near detector fiducial volume, the spectrum $T_{i}^{\mathrm{N}}$ is corrected for cross sections and fiducial mass. For each bin $i$ in true neutrino energy, the energy of the centre of the bin is taken. Using the fiducial 
mass of the near detector $m_{\mathrm{N}}$, the number of protons on target corresponding to the input near detector data $p_{\mathrm{N}}$ and the $\mathrm{CC}$ cross section at the centre of the bin $i$, the flux through the near detector fiducial volume, per proton on target, is

$$
F_{i}^{\mathrm{N}}=\frac{T_{i}^{\mathrm{N}}}{X_{i} m_{\mathrm{N}} p_{\mathrm{N}}} \quad \forall i
$$

\subsubsection{Calculating the Beam Matrix}

Using the decay kinematics of the neutrino parent particles in the Fluka05 simulation of the NuMI beam, the beam matrix is formed which allows a near detector flux to be converted into a far detector flux.

\section{Neutrinos Passing through the Near Detector}

The beam simulation produces as output a file containing every neutrino parent particle in the decay pipe, with all kinematic information about it (such as energy, momentum and point of decay). The primary parents contributing to the neutrino beam are $\pi^{ \pm}, K^{ \pm}$and $\mu^{ \pm}$; these are the only parents considered in the building of the matrix. The relevant decays are

$$
\begin{aligned}
\pi^{ \pm} & \rightarrow \nu_{\mu} / \bar{\nu}_{\mu}+\mu^{ \pm} \\
K^{ \pm} & \rightarrow \nu_{\mu} / \bar{\nu}_{\mu}+\mu^{ \pm} \\
\mu^{ \pm} & \rightarrow e^{ \pm}+\nu_{e} / \bar{\nu}_{e}+\bar{\nu}_{\mu} / \nu_{\mu} .
\end{aligned}
$$

To produce a near detector spectrum from the simulation of the parents, each parent is decayed such that its daughter neutrino travels towards a point $\mathbf{r}_{d}$ chosen randomly within the near detector. (In practice, each parent is decayed towards ten different random points within the near detector to cover a range of possible decay angles which result in a neutrino passing through the detector). The energy of the resultant neutrino is required (in the laboratory frame). 
For the two-body decay of a pion or kaon the neutrino energy in the centre of mass (parent rest) frame $E_{\nu, \mathrm{N}}^{*}$ can be calculated. Let the parent have fourmomentum $q_{\mathrm{p}}$ and rest mass $m_{\mathrm{p}}$, the muon $q_{\mu}$ and $m_{\mu}$, and the neutrino $q_{\nu}$ with its mass neglected.

$$
q_{\mu}=q_{\mathrm{p}}-q_{\nu}
$$

Taking the dot product of each side with itself,

$$
m_{\mu}^{2}=m_{\mathrm{p}}^{2}+2\left(E_{\nu, \mathrm{N}}^{*} m_{\mathrm{p}}-0\right) \quad \Rightarrow \quad E_{\nu, \mathrm{N}}^{*}=\frac{m_{\mu}^{2}-m_{\mathrm{p}}^{2}}{2 m_{\mathrm{p}}}
$$

Moving to the lab frame, if the parent three-momentum is $\mathbf{p}_{\mathrm{p}}$ and the parent decay point $\mathbf{r}_{v}$, the angle $\theta$ between the parent and neutrino directions of flight is

$$
\cos \theta=\frac{\left(\mathbf{r}_{d}-\mathbf{r}_{v}\right) \cdot \mathbf{p}_{\mathrm{p}}}{\left|\mathbf{r}_{d}-\mathbf{r}_{v}\right|\left|\mathbf{p}_{\mathrm{p}}\right|}
$$

The neutrino energy in the laboratory frame can then be found (identifying centre of mass frame four momenta with an asterisk):

$$
\begin{aligned}
q_{\mathrm{p}}^{*} \cdot q_{\nu}^{*} & =q_{\mathrm{p}} \cdot q_{\nu} \\
\Rightarrow \quad E_{\mathrm{p}}^{*} E_{\nu, \mathrm{N}}^{*}-\mathbf{p}_{\mathrm{p}}^{*} \cdot \mathbf{p}_{\nu}^{*} & =E_{\mathrm{p}} E_{\nu, \mathrm{N}}-\mathbf{p}_{\mathrm{p}} \cdot \mathbf{p}_{\nu} \\
\Rightarrow \quad m_{\mathrm{p}} E_{\nu, \mathrm{N}}^{*}-0 & =E_{\mathrm{p}} E_{\nu, \mathrm{N}}-\left|\mathbf{p}_{\mathrm{p}}\right|\left|\mathbf{p}_{\nu}\right| \cos \theta
\end{aligned}
$$

We therefore have

$$
\begin{aligned}
E_{\nu, \mathrm{N}} & =\frac{m_{\mathrm{p}} E_{\nu, \mathrm{N}}^{*}}{E_{\mathrm{p}}-\left|\mathbf{p}_{\mathrm{p}}\right| \cos \theta} \\
& =\frac{E_{\nu, \mathrm{N}}^{*}}{\gamma_{\mathrm{p}}\left(1-\beta_{\mathrm{p}} \cos \theta\right)} .
\end{aligned}
$$

The near detector neutrino flux calculated by the previous stages was binned according to true neutrino energy. The parents decayed above can be collected 
into sets (partial parent fluxes) according to which bin the energy of the resulting near detector neutrino falls into. The random decaying of the parents ensures that each partial parent flux contains the correct distribution of parents required to represent the parents responsible for the neutrinos in that near detector energy bin.

The contribution to the far detector neutrino flux of each partial parent flux can now be calculated. At a distance of $735 \mathrm{~km}$ the far detector subtends a negligible solid angle to a parent particle in the NuMI decay pipe. This limits the range of decay angles at which the parent may emit its daughter neutrino such that it passes through the far detector to a similarly negligible range. The resultant energy of the neutrino at the far detector $\left(E_{\mathrm{F}}\right)$ is therefore constrained through kinematics effectively to a single value. If the angle in the laboratory frame between the parent momentum and the direction of the centre of the far detector is $\theta_{\mathrm{D}}$, the calculation is the same as that for the near detector in equation 7.5:

$$
E_{\mathrm{F}}=\frac{E_{\nu, \mathrm{F}}^{*}}{\gamma_{\mathrm{p}}\left(1-\beta_{\mathrm{p}} \cos \theta_{\mathrm{D}}\right)} .
$$

The probability of the parent decaying in a direction such as to pass through the far detector is related to the angular distribution of the parent decays; the number of decays $N$ as a function of angle $\theta$ between the neutrino and parent momenta. In the parent centre of mass frame $\left(\theta^{*}\right.$ being the angle in this frame between the neutrino momentum and an arbitrary axis), the angular distribution for the decays of spinless pions and kaons is isotropic:

$$
\frac{\mathrm{d} N}{\mathrm{~d} \cos \theta^{*}}=\frac{1}{2}
$$

(normalised such that $\int_{-1}^{1} \frac{\mathrm{d} N}{\mathrm{~d} \cos \theta^{*}} \mathrm{~d} \cos \theta^{*}=1$ ). 


\section{Muon Spin Calculation}

For neutrinos from muon parents the situation is more complicated as the muon is a spin-half particle, so $\frac{\mathrm{d} N}{\mathrm{~d} \cos \theta^{*}}$ differs. It is calculated as follows, but on a first reading of this chapter it suffices for the reader to skip straight to the result at equation 7.9 .

The muons arise from the decays of charged, spinless pions and kaons as in equations 7.2 and 7.3. The neutrino or antineutrino produced during muon production must be left or right handed respectively. The muon spin must therefore be aligned such as to conserve angular momentum:

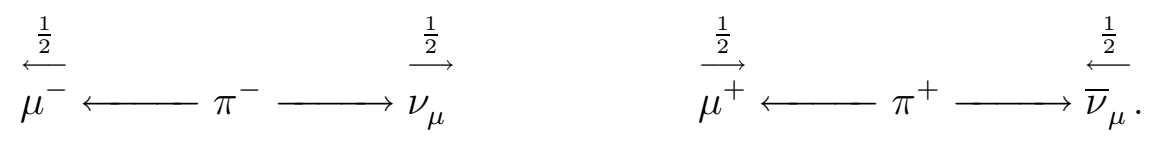

Transforming to the muon rest frame, the $\mu^{-}\left(\mu^{+}\right)$spin is opposite (parallel) to the direction of the incoming pion:

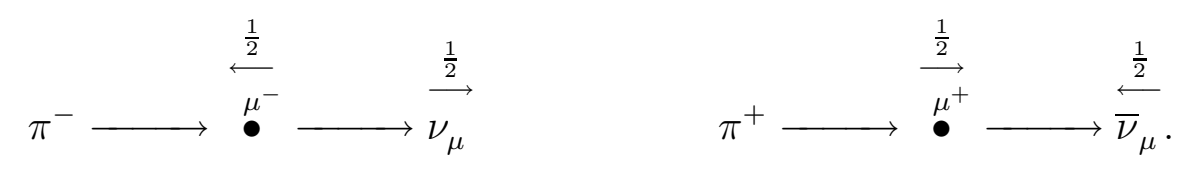

In the muon rest frame, the angular distribution of decays as a function of the angle $\theta_{S}^{*}$ between the muon spin and the momentum of the $\stackrel{(-)}{\nu_{\mu}}$ produced in the muon decay (normalised as equation 7.6) is

$$
\frac{\mathrm{d} N_{\mu^{ \pm}}}{\mathrm{d} \cos \theta_{S}^{*}}=\frac{1}{2}\left(1 \mp \frac{1-2 x}{3-2 x} \cos \theta_{S}^{*}\right)
$$

where $x=E_{\left(\overrightarrow{\nu_{\mu}}\right)} / E_{\left(\overrightarrow{\nu_{\mu}}\right)}^{\max }$, defining $E_{\left(\overrightarrow{\nu_{\mu}}\right)}$ to be the energy of the $\stackrel{\left(\overrightarrow{\nu_{\mu}}\right.}{\text { and }} E_{\left(\overrightarrow{\nu_{\mu}}\right)}^{\max }$ the maximum possible energy of the $\left(\overrightarrow{\nu_{\mu}}\right.$ (namely $\left.m_{\mu} / 2\right)$. See, for example, [176] for a derivation.

Still in the muon rest frame the angle between the $\pi^{ \pm}$and $\stackrel{\left(\vec{\nu}_{\mu}\right.}{\mu}$ momenta is 
labeled $\theta_{\pi}^{*}$. Equation 7.7 shows that for the two cases,

$$
\begin{aligned}
& \mu^{-}: \quad \theta_{\pi}^{*}=180-\theta_{S}^{*} ; \quad \mu^{+}: \quad \theta_{\pi}^{*}=\theta_{S}^{*} ; \\
& \Rightarrow \cos \theta_{\pi}^{*}=-\cos \theta_{S}^{*} \quad \Rightarrow \cos \theta_{\pi}^{*}=\cos \theta_{S}^{*}
\end{aligned}
$$

Thus, in terms of $\theta_{\pi}^{*}$, equation 7.8 becomes the same for both types of muon:

$$
\frac{\mathrm{d} N}{\mathrm{~d} \cos \theta_{\pi}^{*}}=\frac{1}{2}\left(1-\frac{1-2 x}{3-2 x} \cos \theta_{\pi}^{*}\right)
$$

\section{Transformation to the Laboratory Frame}

These decay angular distributions must be transformed from the parent rest frame into the laboratory frame, signified by removing the asterisk from the decay angle:

$$
\frac{\mathrm{d} N}{\mathrm{~d} \cos \theta}=\frac{\mathrm{d} N}{\mathrm{~d} \cos \theta^{*}} \frac{\mathrm{d} \cos \theta^{*}}{\mathrm{~d} \cos \theta} .
$$

Define $\theta$ as the angle between the parent and neutrino momenta as follows:

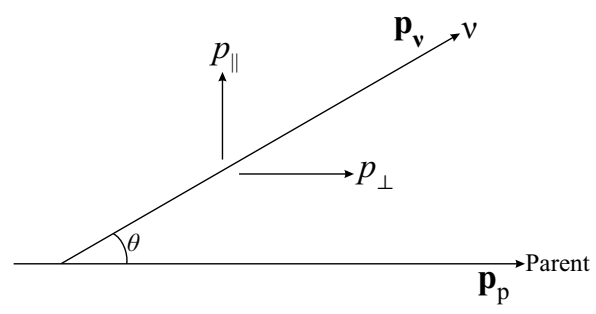

Then (with $E_{\nu}=\left|\mathbf{p}_{\nu}\right|$ )

$$
\cos \theta=\frac{p_{11}}{E_{\nu}} ; \quad \quad \cos \theta^{*}=\frac{p_{11}^{*}}{E_{\nu}^{*}}
$$

Performing the Lorentz transformation between the frames (where the parent has a gamma factor $\gamma_{\mathrm{p}}$ and corresponding $\beta_{\mathrm{p}}$ in the laboratory frame),

$$
p_{\|}^{*}=\gamma_{\mathrm{p}}\left(p_{\|}-\beta_{\mathrm{p}} E_{\nu}\right) ; \quad E_{\nu}^{*}=\gamma_{\mathrm{p}}\left(E_{\nu}-\beta_{\mathrm{p}} p_{\|}\right)
$$


Thus

$$
\cos \theta^{*}=\frac{p_{\|}-\beta_{\mathrm{p}} E_{\nu}}{E_{\nu}-\beta_{\mathrm{p}} p_{\|}}=\frac{\cos \theta-\beta_{\mathrm{p}}}{1-\beta_{\mathrm{p}} \cos \theta} .
$$

So

$$
\begin{aligned}
\frac{\mathrm{d} \cos \theta^{*}}{\mathrm{~d} \cos \theta} & =\frac{1}{1-\beta_{\mathrm{p}} \cos \theta}+\beta \frac{\cos \theta-\beta_{\mathrm{p}}}{\left(1-\beta_{\mathrm{p}} \cos \theta\right)^{2}} \\
& =\frac{1-\beta_{\mathrm{p}}^{2}}{\left(1-\beta_{\mathrm{p}} \cos \theta\right)^{2}} \\
& =\frac{1}{\gamma^{2}} \frac{1}{\left(1-\beta_{\mathrm{p}} \cos \theta\right)^{2}}
\end{aligned}
$$

Thus for pion or kaon decay,

$$
\frac{\mathrm{d} N}{\mathrm{~d} \cos \theta}=\frac{1}{2 \gamma^{2}\left(1-\beta_{\mathrm{p}} \cos \theta\right)^{2}}
$$

For muon decay,

$$
\frac{\mathrm{d} N}{\mathrm{~d} \cos \theta}=\left(1-\frac{1-2 x}{3-2 x} \cos \theta_{\pi}^{*}\right) \frac{1}{2 \gamma^{2}\left(1-\beta_{\mathrm{p}} \cos \theta\right)^{2}}
$$

\section{Neutrinos passing through the Far Detector}

The probability of a parent decaying to emit a neutrino that passes through an arbitrary unit area $A$ on the front face of the far detector fiducial volume is the integral of the relevant normalised angular distribution (equation 7.10 or 7.11 ) over the solid angle subtended by $A$ at the decay point.

$$
P(\nu \text { passes through } A)=\int_{A} \frac{1}{2 \pi} \frac{\mathrm{d} N}{\mathrm{~d} \cos \theta} \mathrm{d} \Omega
$$

where the factor $1 / 2 \pi$ normalises the integral to one, using the fact that the angular distribution is independent of the azimuthal decay angle $\phi$.

Let the solid angle subtended by $A$ at the decay point be $\Omega_{A}$. Due to the large distance between the far detector and the decay point, this solid angle is very 
small. Therefore the angular distribution of the parent decay can be assumed constant over $A$, so the integral can then be approximated by

$$
\begin{aligned}
P(\nu \text { passes through } A) & =\left.\frac{1}{2 \pi} \frac{\mathrm{d} N}{\mathrm{~d} \cos \theta}\right|_{\cos \theta=\cos \theta_{\mathrm{D}}} \Omega_{A} \\
& \left.\approx \frac{1}{2 \pi} \frac{\mathrm{d} N}{\mathrm{~d} \cos \theta}\right|_{\cos \theta=\cos \theta_{\mathrm{D}}} \frac{A}{z_{D}^{2}}
\end{aligned}
$$

where $z_{D}$ is the distance from the decay point to the far detector front face.

\section{Forming the Beam Matrix}

From these equations, the far detector neutrino flux produced by each partial parent flux can be calculated. In a one-dimensional histogram with an $x$-axis of far detector neutrino energy, one entry is made for each parent in the partial flux, at the energy of its neutrino progeny directed towards the far detector, weighted by $P(\nu$ passes through FD fid. vol.). Taking a two-dimensional histogram, with an $x$-axis of near detector neutrino energy and $y$-axis of far detector neutrino energy, each column of the histogram can be filled with the far detector neutrino flux (the aforementioned one-dimensional histogram) produced by the partial parent flux corresponding to the near detector neutrino energy of the relevant bin on the $x$-axis (see figure 7.8). ${ }^{1}$

The sum of the far detector neutrino fluxes in each column provides the total neutrino flux produced by the input simulated parent flux. The aim is to correct this to be consistent with the flux measured at the near detector. Let the partial parent flux corresponding to the near detector true neutrino energy bin $i$ produce a number of neutrinos $n_{i}$ in that energy bin. Let the number of neutrinos measured in that bin (after the transformations performed on the near detector data described earlier in this section) be $m_{i}$. The far detector neutrino flux predicted by

\footnotetext{
${ }^{1}$ The partial parent fluxes in the two-dimensional histogram are strictly $A \times$ (partial parent flux); however the $A$ will be cancelled by the calculation which follows for the near detector partial parent flux.
} 


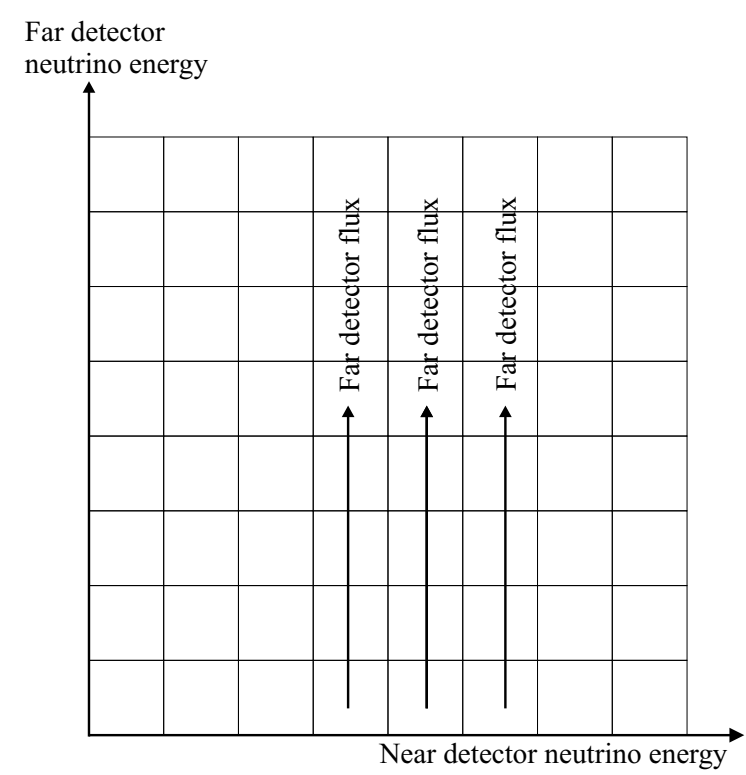

Figure 7.8: A cartoon demonstrating how the far detector neutrino flux produced by each partial parent flux is filled into a two-dimensional histogram, according to the near detector neutrino energy corresponding to the partial parent flux that produced the far detector neutrino flux in question. Each column is scaled to match the measured near detector flux, and the rows then summed to predict the flux at the far detector.

this partial parent flux should be corrected by a scale factor $m_{i} / n_{i}$ before the far detector neutrino fluxes are summed: the $i$ th column of the histogram of figure 7.8 should be scaled by this factor.

The probability that a particular parent (labelling each parent with an index l) decays to produce a neutrino which passes through a slice (in the $x, y$ plane) of the near detector (ND), with an energy such that it is in the $i$ th bin of near detector energy, is

$$
P_{l}(\nu \text { passes through ND slice })=\int_{\text {ND slice }} \frac{1}{2 \pi} \frac{\mathrm{d} N}{\mathrm{~d} \cos \theta} \mathcal{E}(\Omega) \mathrm{d} \Omega
$$

where $\mathcal{E}(\Omega)=1$ if the parent decays producing a neutrino travelling into a solid angle $\Omega$ such that it has an energy in the $i$ th bin, and $\mathcal{E}(\Omega)=0$ if its energy is otherwise.

Through the method adopted of choosing random points within the near detec- 
tor and simulating a parent decay towards that point, this integral is effectively being evaluated through a Monte Carlo technique. As only points producing a neutrino in the $i$ th energy bin are included in the calculation of each partial parent flux, the Monte Carlo integration is being performed by taking points in the solid angle over which $\mathcal{E}(\Omega)=1$. Letting a parent undergo a series of $N_{k}$ decays, each labelled with $k$, towards different near detector points,

$$
P_{l}(\nu \text { passes through ND slice })=\frac{\left.\sum_{k} \frac{1}{2 \pi} \frac{\mathrm{d} N}{\mathrm{~d} \cos \theta}\right|_{\cos \theta_{k}} \Omega_{\mathcal{E}}}{N_{k}}
$$

$\Omega_{\mathcal{E}}$ is the range of solid angles over which $\mathcal{E}(\Omega)=1$, and $1 / 2 \pi$ again provides the normalisation when integrating over the (isotropic) azimuthal angle $\phi$.

In the model used here, each parent is effectively decayed once only; although each parent is actually decayed ten times, each decay is treated as if coming from a different parent, a valid process if a large enough sample of parents are used such that for each parent a large number of kinematically similar parents are also used. Thus $N_{k}=1$ and the sum has only one term. The flux (per unit area) through the near detector slice implied by each parent is then

$$
F_{l}=\frac{P_{l}}{\Omega_{\mathcal{E}}}=\left.\frac{1}{2 \pi} \frac{\mathrm{d} N}{\mathrm{~d} \cos \theta}\right|_{\cos \theta_{l}}
$$

and the corresponding rate of neutrinos passing through an arbitrary area $A$ within the near detector slice is $F_{l} A . A$ is chosen to be the same as that used in the far detector and therefore cancels upon the full application of the beam matrix procedure (see below).

The total rate of neutrinos of an energy in the $i$ th bin passing through an arbitrary area $A$ in the near detector caused by a partial parent flux is then the sum of this probability over all parents in that partial parent flux. Note that each parent is decayed such that its daughter neutrino passes through a point chosen 
at random from the entire fiducial volume: not just one slice. The flux through each slice at a different $z$ will be different, due to the different range of decay angle involved. Thus what is calculated is actually the average of this rate over the whole fiducial volume: which is the correct quantity as it is this average which is measured from the data. ${ }^{2}$ We therefore have an expression for $n_{i}$ :

$$
n_{i}=\left.\sum_{l} \frac{1}{2 \pi} \frac{\mathrm{d} N}{\mathrm{~d} \cos \theta}\right|_{\cos \theta_{l}} A
$$

To incorporate $n_{i}$ into the two-dimensional histogram of figure 7.8 , each bin of the histogram in the $i$ th column is divided by $n_{i}$. The histogram is now normalised such that each column (far detector flux) corresponds to one neutrino in the near detector. (The calculation of the far detector flux in each column included the multiplicative factor $A$ of equation 7.12. When dividing by $n_{i}$, this is cancelled by the factor of $A$ in equation 7.13.) The two dimensional histogram has now become the required beam matrix.

The neutrino and antineutrino beam matrices are shown in figure 7.9. The wide region below $30 \mathrm{GeV}$ comes primarily from the decay of pions, with a small contribution from muon decay. The narrower tail at higher energies arises primarily from the decay of kaons.

\subsubsection{Using the Beam Matrix}

A far detector flux may now be predicted by the application of the beam matrix to the measured near detector flux, $F_{i}^{\mathrm{N}}$, which was calculated by the application to the near detector data of the transformations described earlier in this section.

$F_{i}^{\mathrm{N}}$ is equivalent to $m_{i}$ : the average measured flux of neutrinos in an energy

\footnotetext{
${ }^{2}$ In principle, there will be a small difference in the shape of the neutrino energy spectrum across the near detector. It is possible that some information could be extracted from this to further constrain the knowledge of the neutrino parents, but such studies are beyond the scope of this thesis.
} 

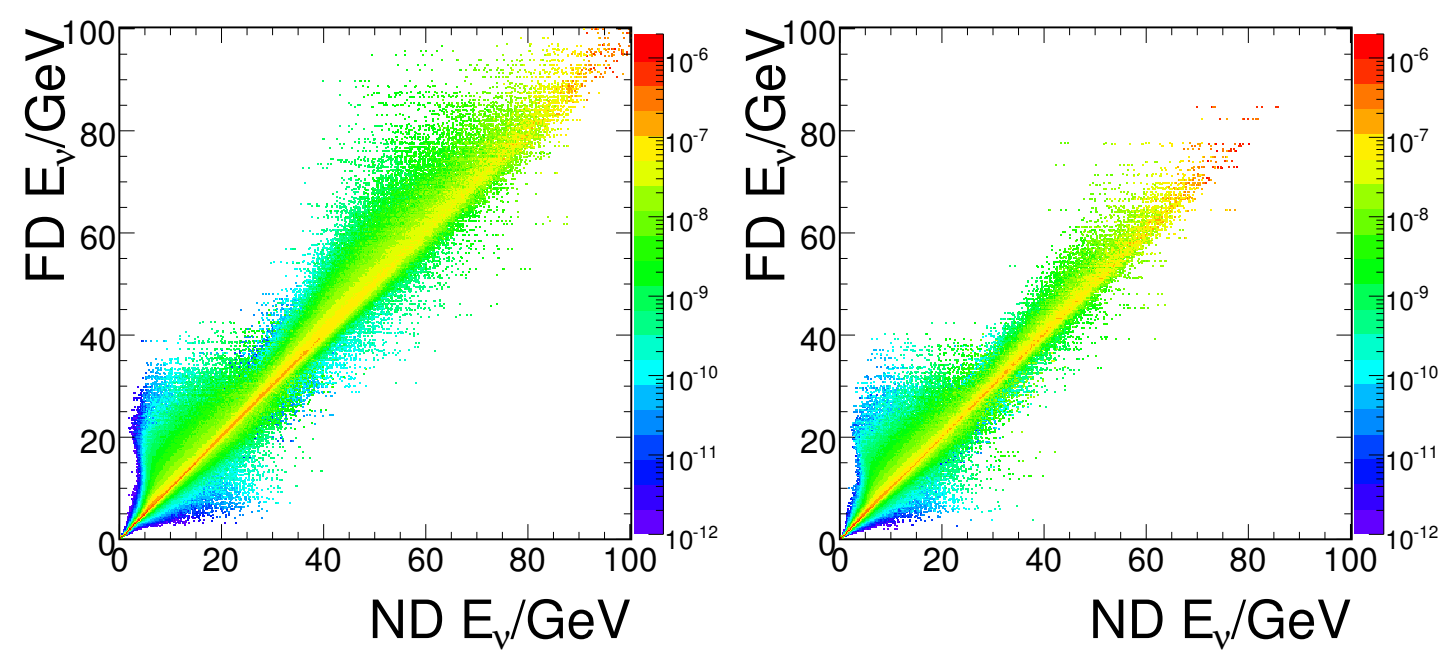

Figure 7.9: The beam matrices used to convert a near detector neutrino (left) and antineutrino (right) flux into a far detector flux.

bin $i$ passing through a slice of the near detector fiducial volume. Each column of the beam matrix should therefore be multiplied by $F_{i}^{\mathrm{N}}$ and the columns then summed to give the predicted far detector flux.

Labelling near detector energy bins with $i$ and far detector energy bins with $j$, and labelling the beam matrix $B_{i j}$, the far detector flux is obtained:

$$
F_{j}^{\mathrm{F}}=\sum_{i} F_{i}^{\mathrm{N}} B_{i j} \quad \forall j
$$

As $F_{i}^{\mathrm{N}}$ was scaled to an exposure of one proton on target, $F_{i}^{\mathrm{F}}$ will be likewise scaled.

\subsubsection{Far Detector Transformations}

The procedure described in $[159,158]$ for the analysis of $\nu_{\mu}$ oscillations performs the near detector transformations described above in the reverse order at the far detector. However, this assumes that the backgrounds (neutral current events in that case) are not affected by oscillations (true for neutral current interactions under the assumption of no sterile neutrinos, an assumption which will be used 
throughout this chapter). The method is slightly different for the $\bar{\nu}_{\mu}$ analysis presented in this thesis as the background in the charged current $\bar{\nu}_{\mu}$ selection consists primarily of charged current $\nu_{\mu}$ events produced by neutrinos which oscillate, potentially with parameters different from the $\bar{\nu}_{\mu}$ signal.

The $\nu_{\mu}$ and $\bar{\nu}_{\mu}$ spectra are separately extrapolated by the method detailed above, yielding fluxes $F_{j}^{\mathrm{F}, \nu_{\mu}}$ and $F_{j}^{\mathrm{F}, \bar{\nu}_{\mu}}$ at the far detector.

Let the far detector fiducial mass be $m_{\mathrm{F}}$ and the number of protons on target corresponding to the required exposure of far detector data be $p_{\mathrm{F}}$. The number of neutrinos interacting in the far detector fiducial volume with a true energy in the $i$ th bin is

$$
T_{i}^{\mathrm{F}, \stackrel{(-)}{\nu_{\mu}}}=F_{i}^{\mathrm{F}, \stackrel{(-)}{\nu_{\mu}}} X_{i}^{\stackrel{(-)}{\nu}} m_{\mathrm{F}} p_{\mathrm{F}}
$$

where $X_{i}^{(-))}$is, as in the near detector case, the $\nu_{\mu}$ or $\bar{\nu}_{\mu}$ cross-section at the centre of the $i$ th bin.

The spectrum can be oscillated at this point, as in figure 7.4; but to illustrate the calculations of all impurities, the oscillations will be returned to at a later point.

The efficiency correction at the far detector (figure 7.10) is calculated:

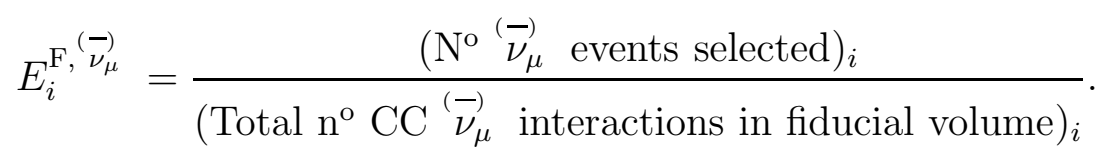

Then the total number of correctly selected events in the $i$ th far detector true energy bin is

$$
T_{i}^{\mathrm{F}, \mathrm{S}, \stackrel{(-)}{\nu})}=T_{i}^{\mathrm{F}, \stackrel{(-)}{\nu})} E_{i}^{\mathrm{F}, \stackrel{(-)}{\nu_{\mu}}} \quad \forall i
$$

The total number of charged current (CC) $\nu_{\mu}$ events wrongly selected as charged current $\bar{\nu}_{\mu}$ events, $K_{i}^{\mathrm{F}, \nu_{\mu}}$ (and vice-versa), can be calculated. A wrong-sign 


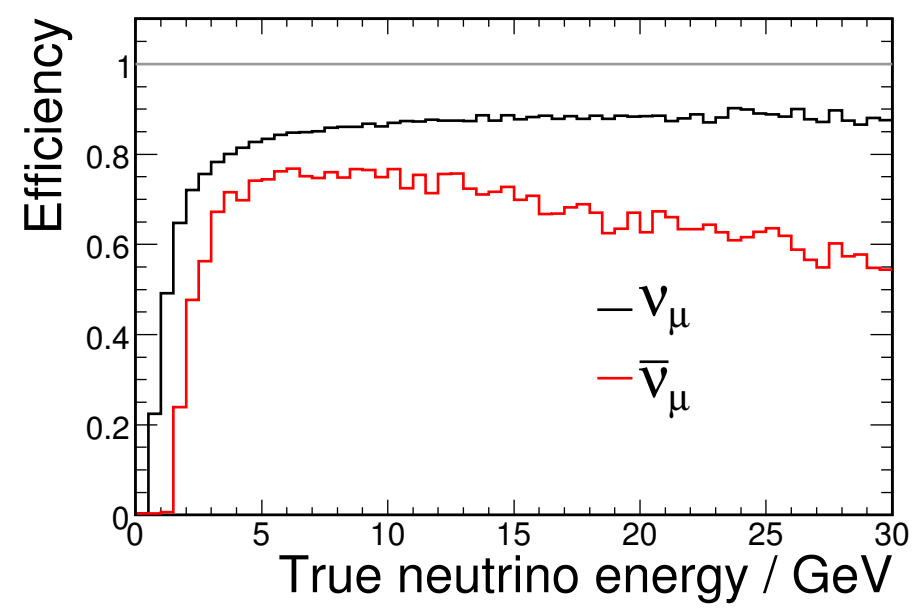

Figure 7.10: The far detector efficiency correction.

efficiency correction (figure 7.11) is defined:

$$
W_{i}^{\mathrm{F}, \nu_{\mu}}=\frac{\left(\mathrm{N}^{\mathrm{o}} \mathrm{CC} \nu_{\mu} \text { events selected as } \mathrm{CC} \bar{\nu}_{\mu} \text { events }\right)_{i}}{\left(\text { Total } \mathrm{n}^{\mathrm{o}} \mathrm{CC} \nu_{\mu} \text { interactions in fiducial volume }\right)_{i}}
$$

and vice-versa for charged current $\bar{\nu}_{\mu}$ events. Then

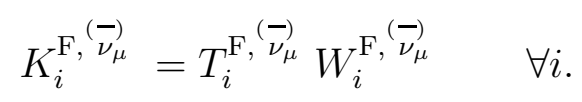

\section{Neutral Current Background}

At this point a digression is necessary to calculate the neutral current impurities in the far detector predictions. On a first reading the reader can skip this section, missing out the details of the calculations leading to the reconstructed energy spectrum of neutral current events $\left(Z_{i}^{\mathrm{F}, \stackrel{(-)}{\nu_{\mu}}}\right)$, which will be added into the final prediction.

Impurities are calculated as a function of reconstructed neutrino energy, in unoscillated Monte Carlo. Thus an unoscillated, reconstructed energy far detector predicted spectrum must be calculated.

As an analogue to the near detector reconstructed to true energy matrix, the 


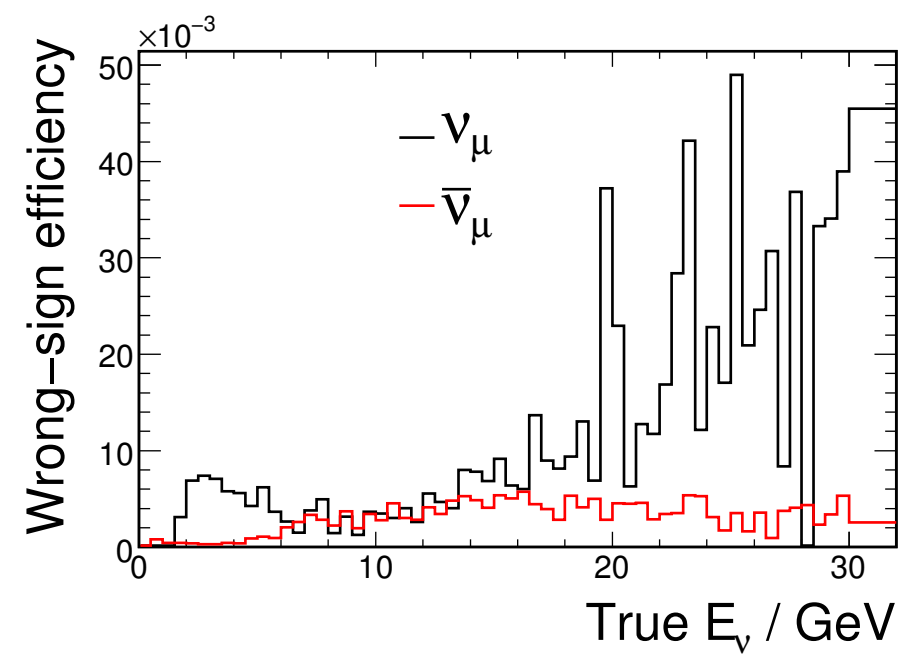

Figure 7.11: The efficiency for neutrino (black) and antineutrino (red) events to be wrongly reconstructed and selected as antineutrino or neutrino events at the far detector.

far detector true to reconstructed energy matrix $\mathcal{M}_{i j}^{\mathrm{F}},\left(\overrightarrow{\nu_{\mu}}\right)$ is formed for both $\nu_{\mu}$ and $\bar{\nu}_{\mu}$ events (figure 7.12). Letting $i$ refer to bins of true neutrino energy and $j$ to bins of reconstructed neutrino energy, a two-dimensional histogram is filled with all selected charged current $\stackrel{(-)}{\nu_{\mu}}$ Monte Carlo events, as in the near detector. The sum of the bins in each column (this time a column in true neutrino energy) is again required to be unity, as in equation 7.1. This ensures that one neutrino event in true energy, when acted upon by the matrix, produces a total of one neutrino event in the reconstructed energy spectrum.

The matrix acts to produce the far detector, unoscillated reconstructed energy spectrum (with no impurities):

$$
U_{j}^{\mathrm{F}, \mathrm{P}, \stackrel{(-)}{\nu \mu})}=\sum_{i} T_{i}^{\mathrm{F}, \mathrm{S}, \stackrel{(-)}{\nu \mu}} \mathcal{M}_{i j}^{\mathrm{F}, \stackrel{(-)}{\nu \mu}} \quad \forall j .
$$

The total far detector purity vector (figure 7.13) can be defined from Monte Carlo for the case of no oscillations: 

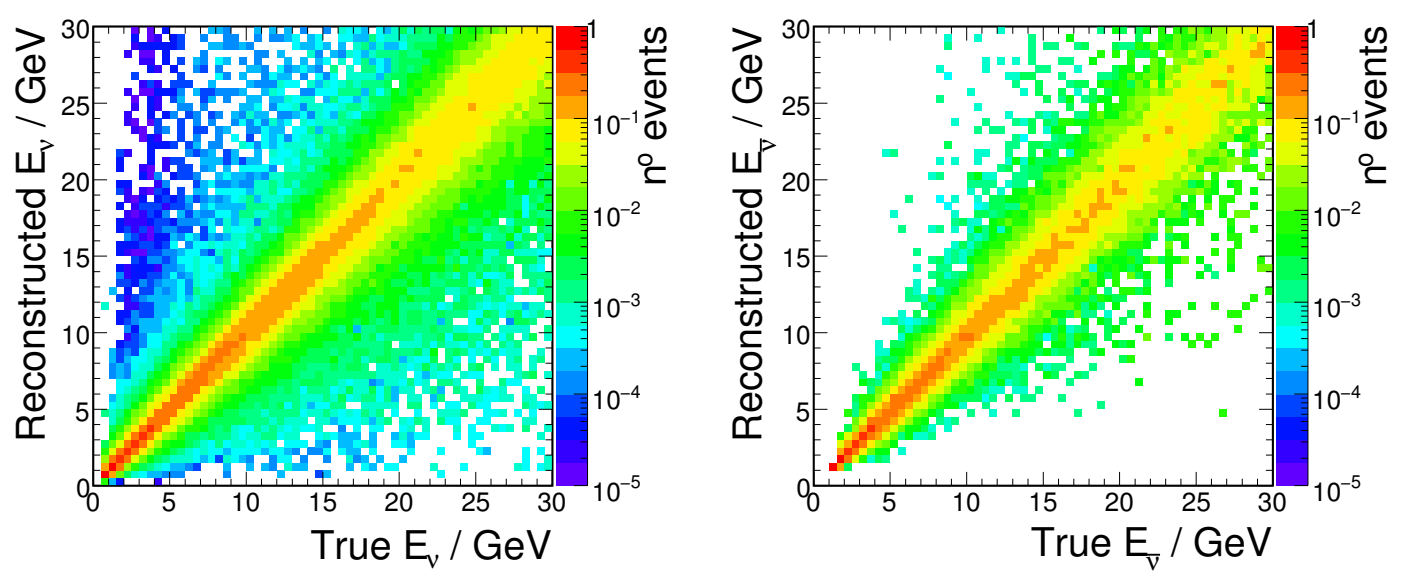

Figure 7.12: The true to reconstructed energy conversion matrices applied at the far detector, for neutrinos (left) and antineutrinos (right).

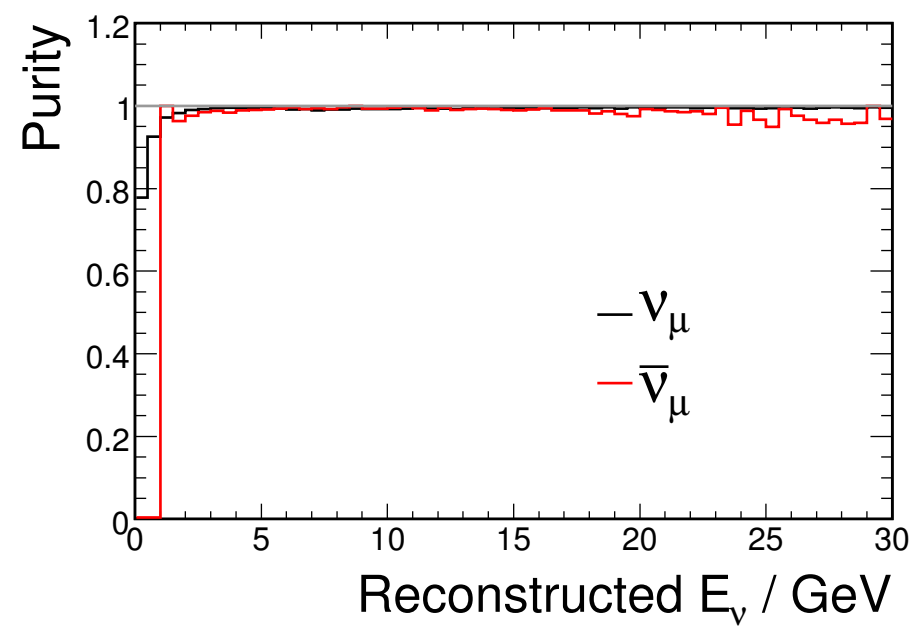

Figure 7.13: The far detector purity correction, relative to an unoscillated energy spectrum. 


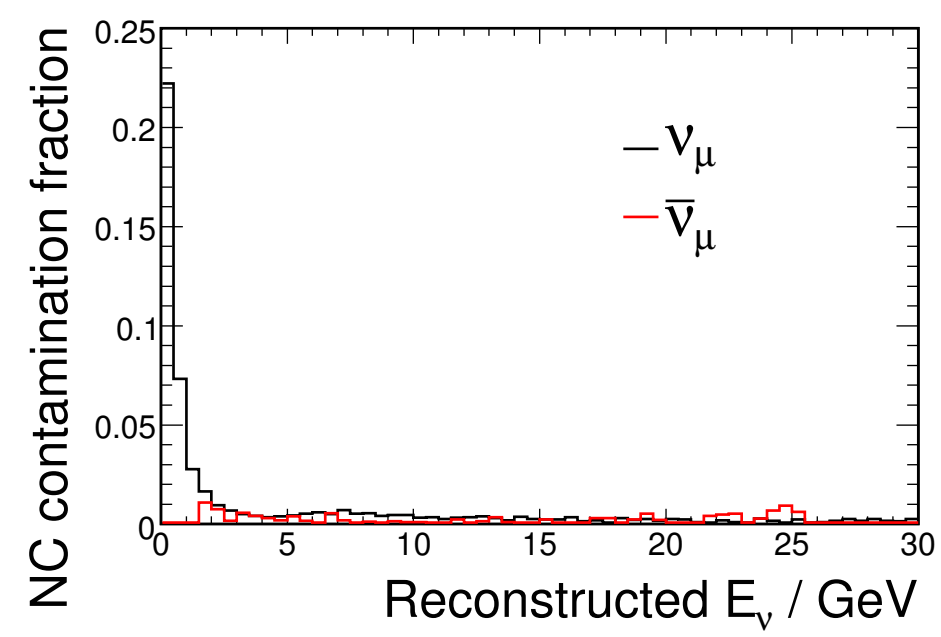

Figure 7.14: The far detector neutral current (NC) contamination fraction, relative to an unoscillated energy spectrum.

$$
P_{i}^{\mathrm{F}, \stackrel{(-)}{\nu})}=\frac{\left(\mathrm{N}^{\mathrm{o}} \text { true } \mathrm{CC} \stackrel{(-)}{\nu_{\mu}} \text { events selected, no oscillations }\right)_{i}}{\left(\text { Total } \mathrm{n}^{\mathrm{o}} \text { events selected as } \mathrm{CC} \stackrel{(-)}{\nu_{\mu}} \text { events, no oscillations }\right)_{i}}
$$

The complete, unoscillated far detector prediction is then

$$
U_{i}^{\mathrm{F}, \stackrel{(-)}{\nu})}=\frac{U_{i}^{\mathrm{F}, \mathrm{P}, \stackrel{(-)}{\nu_{\mu}}}}{P_{i}^{\mathrm{F}, \stackrel{(-)}{\nu_{\mu}}}} \quad \forall i
$$

A neutral current (NC) contamination vector, relative to the no oscillation far detector spectrum, is calculated (figure 7.14):

$$
N_{i}^{\mathrm{F}, \stackrel{(-)}{\nu_{\mu}}}=\frac{\left(\mathrm{N}^{\mathrm{o}} \mathrm{NC} \text { events selected as } \mathrm{CC} \stackrel{\left(-\vec{\nu}_{\mu}\right.}{\nu_{\mu}} \text { events }\right)_{i}}{\left(\text { Total } \mathrm{n}^{\mathrm{o}} \text { events selected as } \mathrm{CC} \stackrel{(-)}{\nu_{\mu}} \text { events, no oscillations }\right)_{i}}
$$

This allows the spectrum of the neutral current events selected as charged current $\stackrel{(-)}{\nu_{\mu}}$ events to be calculated:

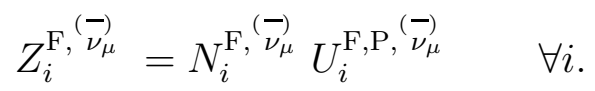




\section{Charged Current Signal}

This ends the digression and the process now moves back to the true energy spectra

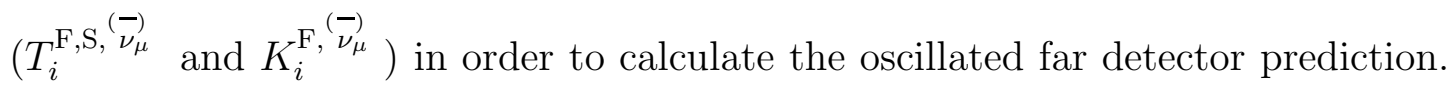
Oscillations are applied to the spectra, according to the energy at the centre of the true energy bin, $E_{\left(\overrightarrow{\nu_{\mu}}, i\right.}$, with $L$ the distance from proton target to far detector:

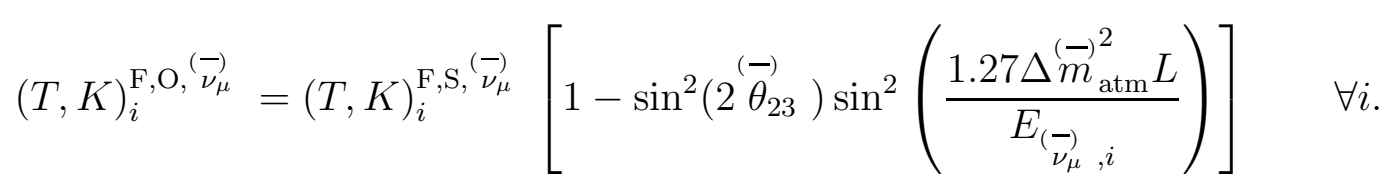

Note that as this is simply a scale factor on the bin contents, it can be done (as in figure 7.4) interchangeably with the efficiency correction.

The same true to reconstructed energy matrices as above, $\mathcal{M}_{i j}^{\mathrm{F},}\left(\overrightarrow{\nu_{\mu}}\right)$ (figure 7.12 ), are used to convert the oscillated signal distributions to oscillated, reconstructed energy distributions. These matrices are unaffected by oscillations as each column of true energy is normalised such that its bins sum to unity. Oscillations would affect the relative number of entries in each column, an effect which the normalisation has negated. A similar true to reconstructed energy matrix, $\tilde{\mathcal{M}}_{i j}^{\mathrm{F}},\left(\vec{\nu}_{\mu}\right)$, is created for each spectrum of charged current $\stackrel{\left(\nu_{\mu}\right.}{\nu_{\mu}}$ events selected in the wrong spectrum $\left(K_{i}^{\mathrm{F},{ }^{(-)}}{ }_{\mu}\right.$; figure 7.15$)$. The reconstructed energy spectra of the correctly selected, oscillated charged current $\stackrel{(-)}{\nu_{\mu}}$ events $\left(R_{j}^{\mathrm{F}, \mathrm{P}} \stackrel{\left(\overrightarrow{\nu_{\mu}}\right.}{)}\right)$ and the incorrectly selected charged current $\stackrel{(-)}{\nu_{\mu}}$ events $\left.\left(C_{i}^{\mathrm{F},} \stackrel{(-)}{\nu}\right)^{2}\right)$ are calculated:

$$
\begin{aligned}
& \left(R^{\mathrm{P}}, C\right)_{j}^{\mathrm{F}}, \stackrel{(\vec{\nu})}{\nu_{\mu}}=\sum_{i}(T, K)_{i}^{\mathrm{F}, \mathrm{O}} \stackrel{(\vec{\nu})}{\nu_{\mu}}(\mathcal{M}, \tilde{\mathcal{M}})_{i j}^{\mathrm{F},}\left(\vec{\nu}_{\mu} \quad \forall j\right. \\
& R_{j}^{\mathrm{P}, \mathrm{F}, \stackrel{(-)}{\nu})}=\sum_{i} T_{i}^{\mathrm{F}, \mathrm{O} \stackrel{(-)}{\nu})} \mathcal{M}_{i j}^{\mathrm{F}, \stackrel{(-)}{\nu})} \quad \forall j . \\
& C_{j}^{\mathrm{F}, \stackrel{(-)}{\nu})}=\sum_{i} K_{i}^{\mathrm{F}, \mathrm{O} \stackrel{(-)}{\nu_{\mu}}} \tilde{\mathcal{M}}_{i j}^{\mathrm{F}, \stackrel{(-)}{\nu})} \quad \forall j
\end{aligned}
$$



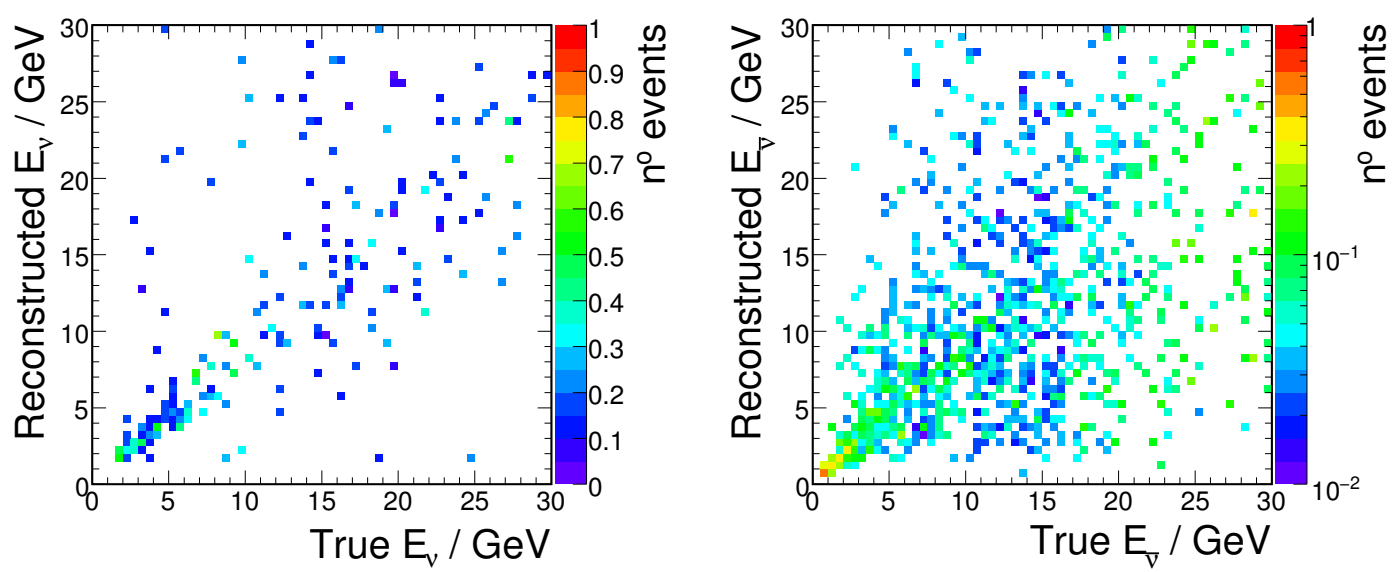

Figure 7.15: The true to reconstructed energy conversion matrices applied to neutrinos wrongly identified as antineutrinos (left) and vice versa (right) at the far detector.

\section{Tau Neutrino Background}

A small background exists of events from the charged current interactions of the $\stackrel{\left(\vec{\nu}_{\mu}\right.}{ }$ which have oscillated into $\stackrel{(}{\nu}_{\tau}$, the background coming predominantly from interactions by the process

$$
{ }^{56} \mathrm{Fe}+\nu_{\tau} \rightarrow X+\left(\tau^{-} \rightarrow \mu^{-}+\nu_{\tau}+\bar{\nu}_{\mu}\right)
$$

and similarly for $\bar{\nu}_{\tau}$. This background is predicted using a process similar to that by which the signal is predicted. This section can be omitted on a first reading.

Moving back to the $\stackrel{(-)}{\nu_{\mu}}$ flux predicted at the far detector, $F_{j}^{\mathrm{F}}, \stackrel{(\vec{\nu})}{\mu}$, the fraction of this which has oscillated to tau neutrinos can be calculated

$$
F_{i}^{\mathrm{F},} \stackrel{(-))}{\nu_{\tau}}=F_{i}^{\mathrm{F},} \stackrel{(-))}{\nu_{\mu}}\left[\sin ^{2}\left(2 \vec{\theta}_{23}\right) \sin ^{2}\left(\frac{1.27 \Delta m_{\mathrm{matm}}^{(-)^{2}} L}{E_{\left(-\vec{\nu}_{\mu}, i\right.}}\right)\right] \quad \forall i
$$

The spectrum of charged current interactions at the far detector can be found through multiplication by the charged current $\bar{\nu}_{\tau}$ cross sections, fiducial mass and 


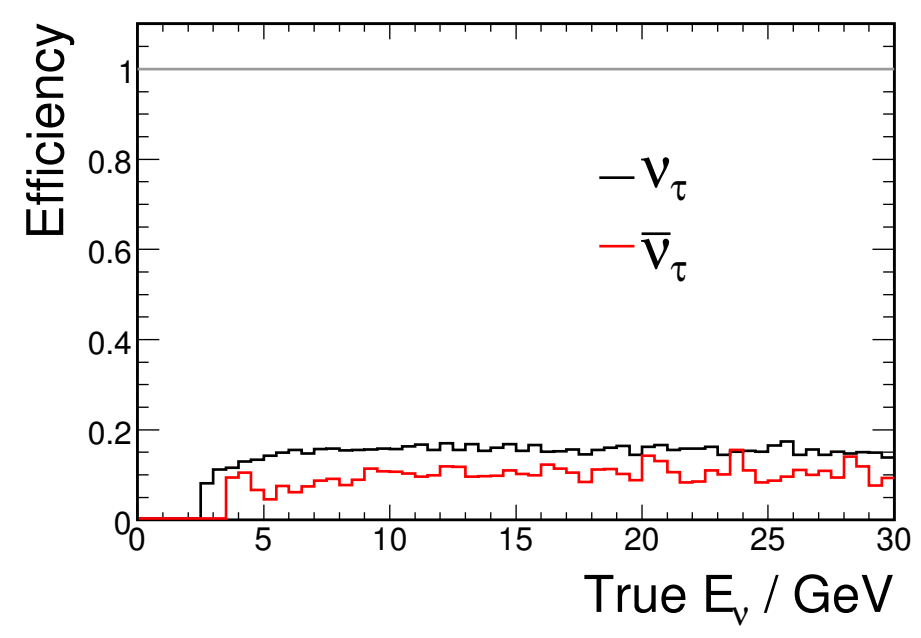

Figure 7.16: The far detector $\stackrel{(-)}{\nu_{\tau}}$ efficiency correction.

exposure:

$$
T_{i}^{\mathrm{F}, \stackrel{(-)}{\nu_{\tau}}}=F_{i}^{\mathrm{F}, \stackrel{(-)}{\nu_{\tau}}} X_{i}^{(-))} m_{F} p_{F} \quad \forall i
$$

A tau neutrino selection and reconstruction efficiency correction, $E_{i}^{\mathrm{F}}, \stackrel{(-))}{\nu_{\tau}}$ calculated as for the $\stackrel{(-)}{\nu_{\mu}}$ case (equation 7.14 ) can be applied to calculate the spectrum of

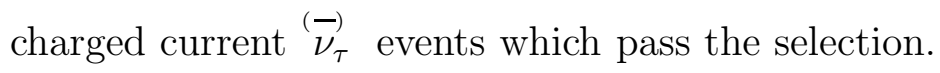

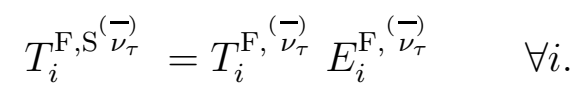

This efficiency correction is shown in figure 7.16 and never goes above 0.2 . This is expected as the branching fraction of tau leptons into muons is $\sim \frac{1}{5}$.

Finally, a true to reconstructed energy matrix $\mathcal{M}_{i j}^{\mathrm{F}},\left(\overrightarrow{\nu_{\tau}}\right)$ (figure 7.17 ) is produced as in the $\left(\overrightarrow{\nu_{\mu}}\right)$ case, to convert the $\left(\vec{\nu}_{\tau}\right.$ true energy spectrum in bins $i$ to a reconstructed energy spectrum in bins $j, R_{j}^{\mathrm{F}},\left(\overrightarrow{\nu_{\tau}}\right)$. 

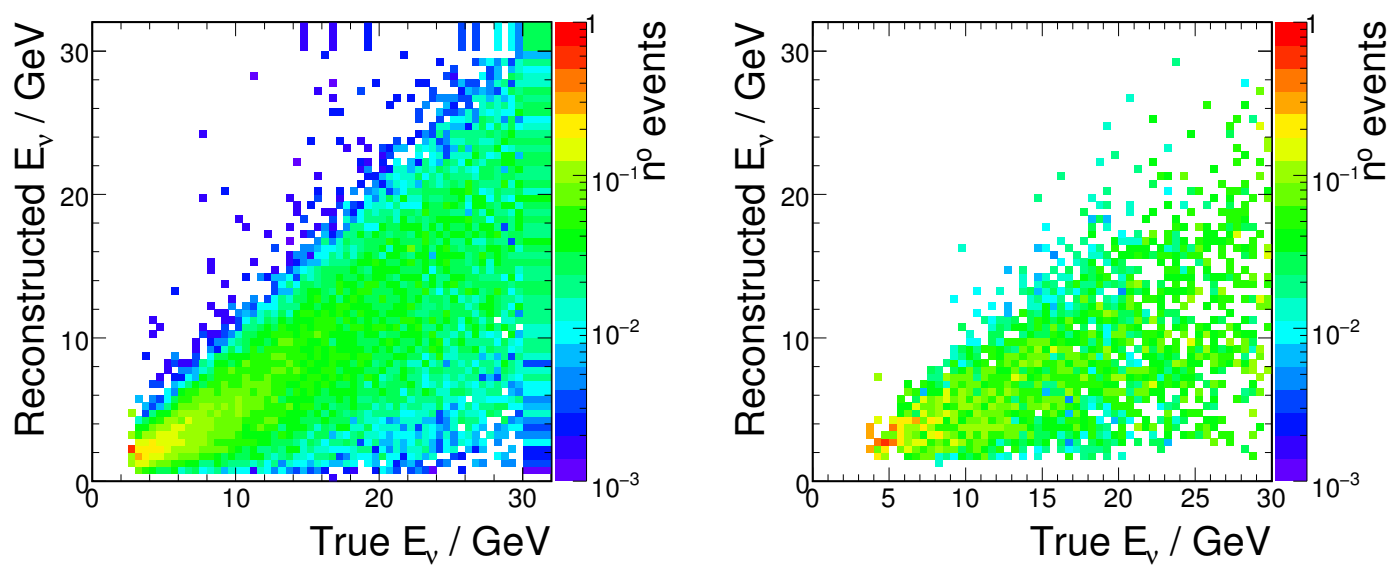

Figure 7.17: The true to reconstructed energy conversion matrices applied to the $\nu_{\tau}$ (left) and $\bar{\nu}_{\tau}$ (right) predicted spectra.

\section{Complete Prediction}

It now remains to add the impurities into the predicted signal distributions, to obtain the total far detector predicted spectra:

$$
\begin{array}{ll}
R_{i}^{\mathrm{F}, \nu_{\mu}}=R_{i}^{\mathrm{F}, \mathrm{P}, \nu_{\mu}}+C_{i}^{\mathrm{F}, \bar{\nu}_{\mu}}+Z_{i}^{\mathrm{F}, \nu_{\mu}}+R_{i}^{\mathrm{F}, \nu_{\tau}} & \forall i, \\
R_{i}^{\mathrm{F}, \bar{\nu}_{\mu}}=R_{i}^{\mathrm{F}, \mathrm{P}, \bar{\nu}_{\mu}}+C_{i}^{\mathrm{F}, \nu_{\mu}}+Z_{i}^{\mathrm{F}, \bar{\nu}_{\mu}}+R_{i}^{\mathrm{F}, \bar{\nu}_{\tau}} & \forall i .
\end{array}
$$

\subsection{Cross-Checking the Extrapolation}

To ensure the extrapolation is correctly implemented, the most basic check is to extrapolate a near detector Monte Carlo fake data spectrum, using that same Monte Carlo to create the near detector extrapolation matrices. The resulting far detector prediction should be compared to a similar fake data spectrum made from the same far detector Monte Carlo as is used to make the far detector extrapolation matrices. The results of this exercise are shown in figure 7.18. The agreement should not be expected to be exact: the near detector Monte Carlo is, at some 

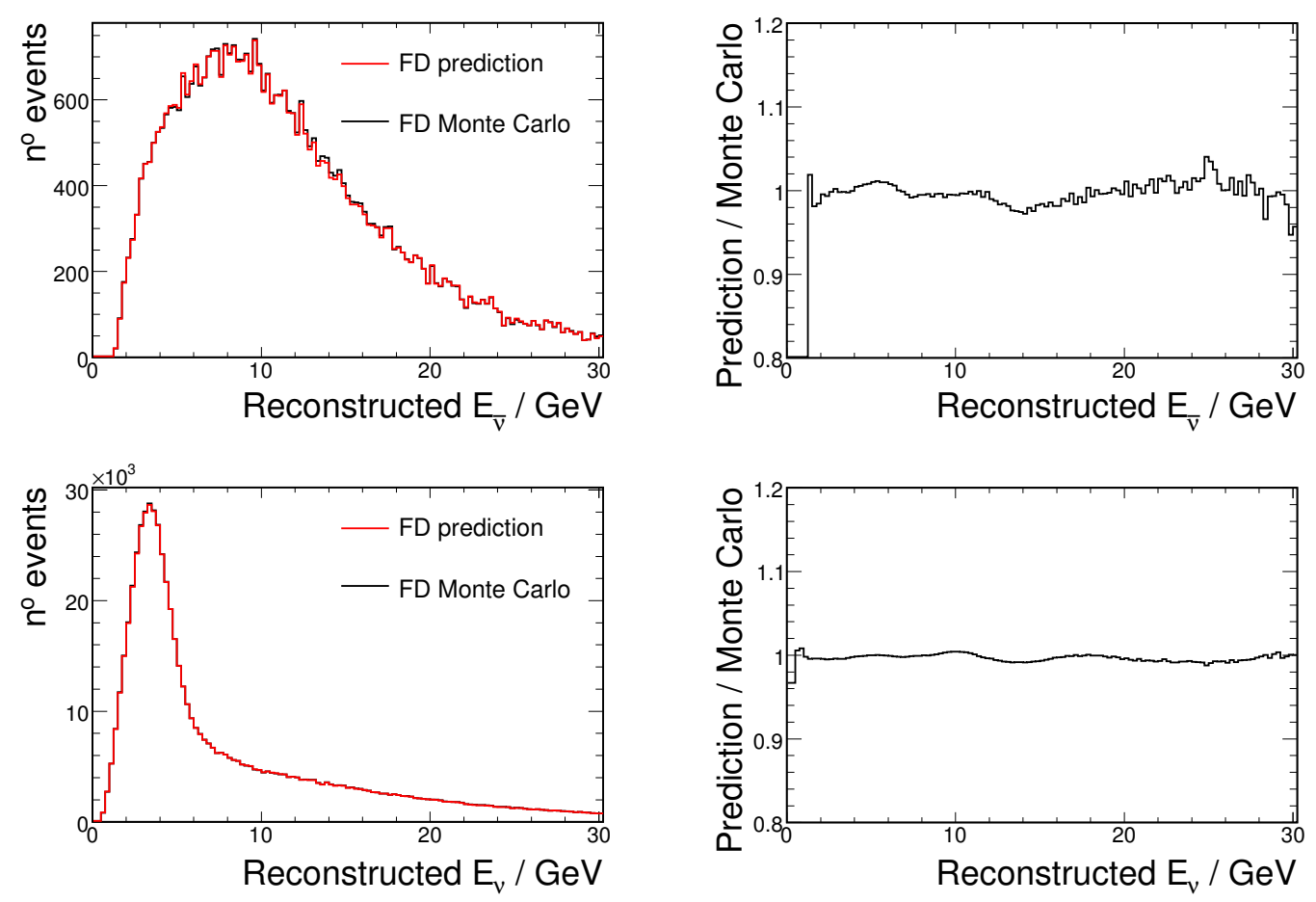

Figure 7.18: Left: the raw far detector Monte Carlo (black) and the prediction obtained by applying the beam matrix extrapolation to the near detector Monte Carlo (red) for the CC $\bar{\nu}_{\mu}$ (top) and $\nu_{\mu}$ (bottom) spectra. Right: the corresponding ratios of the prediction divided by the far detector Monte Carlo. 
level, statistically independent from the far detector Monte Carlo; the beam matrix is furthermore independent from them both. The $\nu_{\mu}$ prediction shows fluctuations at the level of $1 \%$; the $\bar{\nu}_{\mu}$ prediction (for which the Monte Carlo statistics are much lower) fluctuates at the $3 \%$ level. These fluctuations are much smaller than the statistics of the far detector data, so the agreement is considered easily adequate for the purposes of the analysis.

\subsection{Far Detector Fitting}

The far detector spectra predicted by the above extrapolation methods are compared to the data using the following likelihood function [58]:

$$
-2 \ln \lambda(\mathbf{W})=2 \sum_{i=1}^{N}\left[m_{i}(\mathbf{W})-d_{i}+d_{i} \ln \left(\frac{d_{i}}{m_{i}(\mathbf{W})}\right)\right]
$$

where $d_{i}$ is the number of data events in the $i$ th bin of reconstructed energy, and $m_{i}(\mathbf{W})$ the corresponding number of predicted events ( $\mathbf{W}$ representing the combination of oscillation parameters being measured). For the antineutrino analysis, only the antineutrino prediction and data are compared in forming the likelihood $\lambda$. The quantity $-2 \ln \lambda(\mathbf{W})$ is minimised (using the Minuit minimisation package [177]) to obtain the best fit oscillation parameters.

\subsection{Extrapolation of Near Detector Data}

Data from the two data-taking periods are extrapolated and fitted separately due to the differing Monte Carlo needed in each case (affecting all the histograms and matrices used for the extrapolation). The far detector predictions for the two periods are shown in figure 7.19 (with no oscillations applied), along with the far detector Monte Carlo corresponding to those periods. The prediction for 

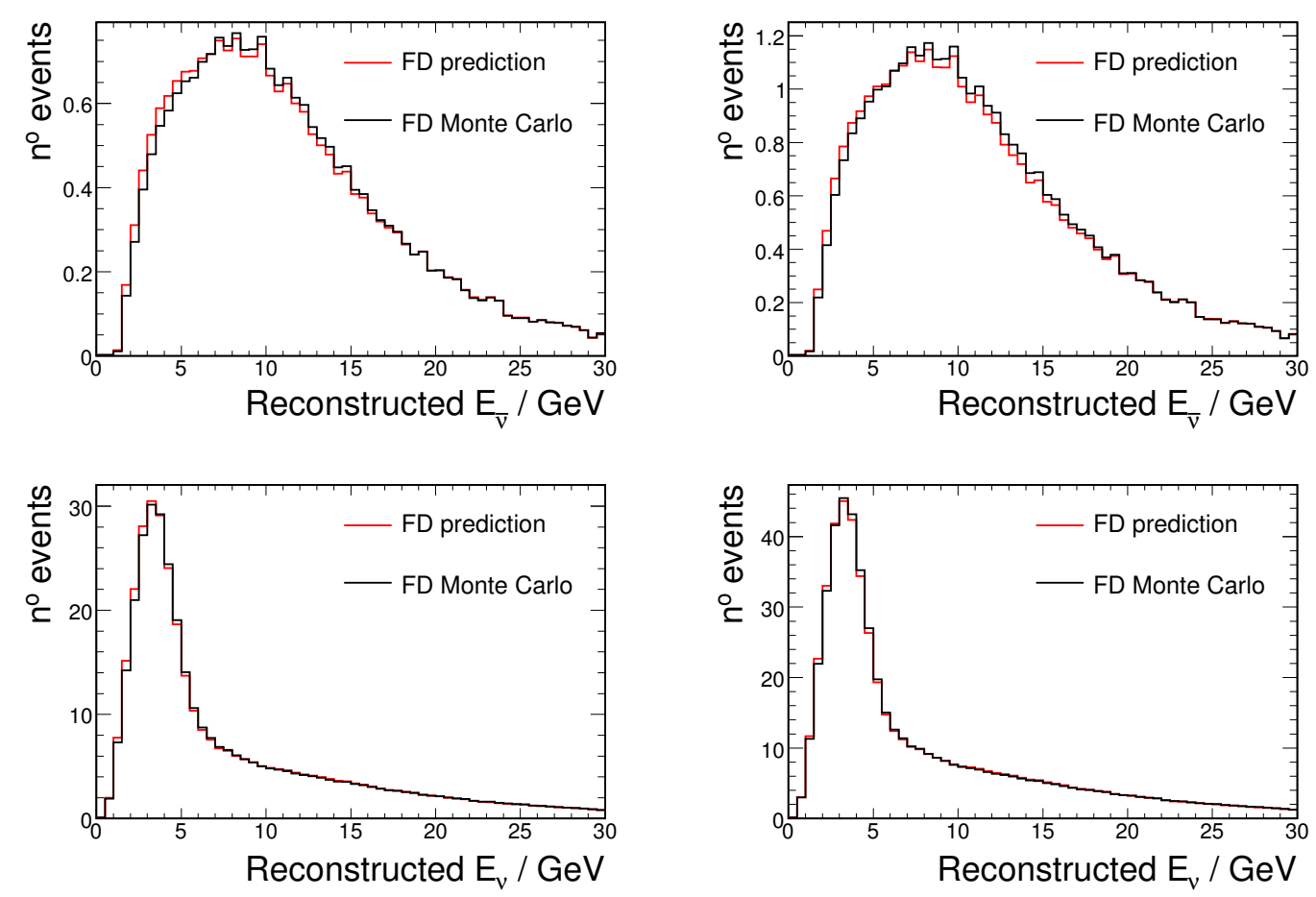

Figure 7.19: The far detector predictions obtained through extrapolating the near detector data for the $\mathrm{CC} \bar{\nu}_{\mu}$ (top) and $\nu_{\mu}$ (bottom) spectra, for the first and second data taking periods (left and right respectively). 


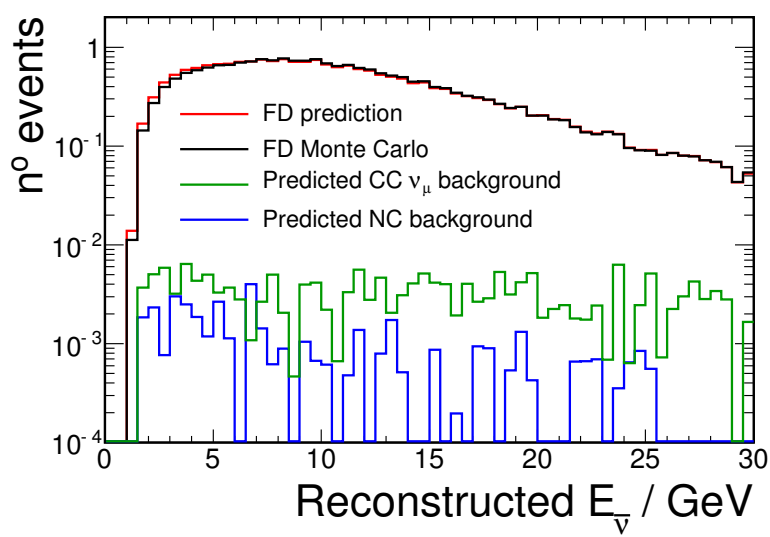

Figure 7.20: The CC $\bar{\nu}_{\mu}$ far detector prediction obtained through extrapolating the near detector data (red), shown with the far detector Monte Carlo spectrum (black) on a logarithmic $y$-axis scale in order to show the predicted background contributions (wrong-sign $\mathrm{CC}$ in green, $\mathrm{NC}$ in blue).

the second period is shown with a logarithmic $y$-axis in figure 7.20 to make the predicted backgrounds visible.

\subsection{Summary}

A method of extrapolating a measured reconstructed energy spectrum at the near detector to give a predicted, oscillated far detector spectrum has been adapted from previous MINOS analyses and developed for use in an antineutrino analysis allowing for the potential of differing $\nu_{\mu}$ and $\bar{\nu}_{\mu}$ oscillation parameters. This has required the introduction of a novel method of dealing with wrong-sign charged current backgrounds. The prediction will be compared to the far detector data using a likelihood function, which will be minimised to find the best fit oscillation parameters.

The extrapolation has been cross-checked through the application to the MINOS Monte Carlo simulation. Finally, the extrapolation procedure has been applied to the near detector data to obtain the far detector prediction in the case of no oscillations. 


\begin{tabular}{|l|l|}
\hline Symbol & \\
\hline$P^{\mathrm{N}}$ & ND purity correction \\
$\mathcal{M}^{\mathrm{N}}$ & ND reconstructed to true energy correction matrix \\
$E^{\mathrm{N}}$ & ND efficiency correction \\
$B$ & Beam matrix \\
$E^{\mathrm{F}}$ & FD efficiency correction \\
$W^{\mathrm{F}}$ & FD wrong-sign efficiency correction \\
$\mathcal{M}^{\mathrm{F}}$ & FD true to reconstructed energy correction matrix \\
$\tilde{\mathcal{M}}^{\mathrm{F}}$ & FD true to reconstructed energy correction matrix for wrong-sign events \\
$P^{\mathrm{F}}$ & FD purity correction \\
$N^{F}$ & NC contamination vector \\
\hline \hline$R^{\mathrm{N}}$ & Measured near detector reconstructed energy spectrum \\
$R^{\mathrm{N}, \mathrm{P}}$ & ND reconstructed energy spectrum after purity correction \\
$T^{\mathrm{N}, \mathrm{S}}$ & ND selected true energy spectrum \\
$T^{\mathrm{N}}$ & ND true energy spectrum (corrected for all efficiencies) \\
$F^{\mathrm{N}}$ & ND flux \\
$F^{\mathrm{F}}$ & FD flux \\
$T^{\mathrm{F}}$ & FD true energy spectrum (corrected for all efficiencies) \\
$T^{\mathrm{F}, \mathrm{S}}$ & FD selected true energy spectrum \\
$K^{\mathrm{F}}$ & FD wrong-sign selected true energy spectrum \\
$U^{\mathrm{F}, \mathrm{P}}$ & FD reconstructed energy unoscillated spectrum (no impurities) \\
$U^{\mathrm{F}}$ & FD reconstructed energy unoscillated spectrum \\
$Z^{\mathrm{F}}$ & FD spectrum of NC events \\
$T^{\mathrm{F}, \mathrm{O}}$ & FD selected true energy spectrum with oscillations \\
$R^{\mathrm{F}, \mathrm{P}}$ & FD reconstructed energy spectrum with oscillations (no impurities) \\
$C^{\mathrm{F}}$ & FD reconstructed energy spectrum of wrong-sign events \\
$R^{\mathrm{F}}$ & Complete FD prediction \\
\hline
\end{tabular}

Table 7.1: Symbols used for the spectra and correction matrices of the beam matrix extrapolation method. 


\section{Chapter 8}

\section{Systematic Uncertainties}

\subsection{Introduction}

A number of systematic uncertainties exist which could cause an error in the best fit to the antineutrino oscillation parameters, or cause a fake antineutrino appearance signal. These uncertainties could all result in an error in the simulated far detector spectrum through shifts in the event energies, errors in the number of selected signal events, or errors in the background estimation. This chapter lists all of the areas of uncertainty expected to contribute to the antineutrino analyses presented in this thesis, and estimates the effect of the uncertainties on those analyses.

\subsection{Hadronic Energy Measurement}

The conversion factor between the detector response to a hadronic shower and the corresponding measured hadronic energy is set by the calibration chain discussed in section 4.2. There are two ways in which this conversion factor could be incorrect. The absolute conversion factor (between detector response and absolute hadronic energy) could be incorrect in the same way at both detectors. Additionally, there 
is the possibility that the relative conversion factor between the two detectors could be incorrect (a difference between the 'true' factor and that used to generate the Monte Carlo). The $1 \sigma$ uncertainty on the absolute factor is a $5.7 \%$ scale of calorimetric shower energies. The uncertainties on the relative factors are $2.4 \%$ in the far detector and $2.3 \%$ in the near detector.

The calibrations used to obtain the hadronic energy scale were performed using cosmic muons. Any errors in the modelling of hadronic showers produced in the neutrino interactions (performed using the NEUGEN package [149]) in the detector simulation will therefore cause an additional, uncorrected data to Monte Carlo disagreement. Estimates of this effect have been made [178] by varying the cross sections, branching ratios and formation lengths involved in shower generation, and observing the effect on the hadronic energies of $\mathrm{CC} \nu_{\mu}$ events. These results are taken to be applicable to $\bar{\nu}_{\mu} \mathrm{CC}$ events. The study estimates an energy-dependent uncertainty on measured shower energies: for showers of energies $<0.5 \mathrm{GeV}$, this uncertainty is a $8.2 \%$ scale factor, the uncertainty dropping to $\sim 3.5 \%$ for showers of energies above $6 \mathrm{GeV}$.

The uncertainties from calibration and hadronic modelling are thought to be uncorrelated, and are therefore added in quadrature to give the energy dependence shown in figure 8.1. Also shown in this figure is an exponential parameterisation of the uncertainty as a function of shower energy $E_{\text {shw }}$ :

$$
\text { Uncertainty }=7 \%+\mathrm{e}^{-E_{\mathrm{shw}} /(1.5 \mathrm{GeV})} \times 4 \% \text {. }
$$

This parameterisation is used in the estimation of the effect of the uncertainty on the antineutrino analysis. 


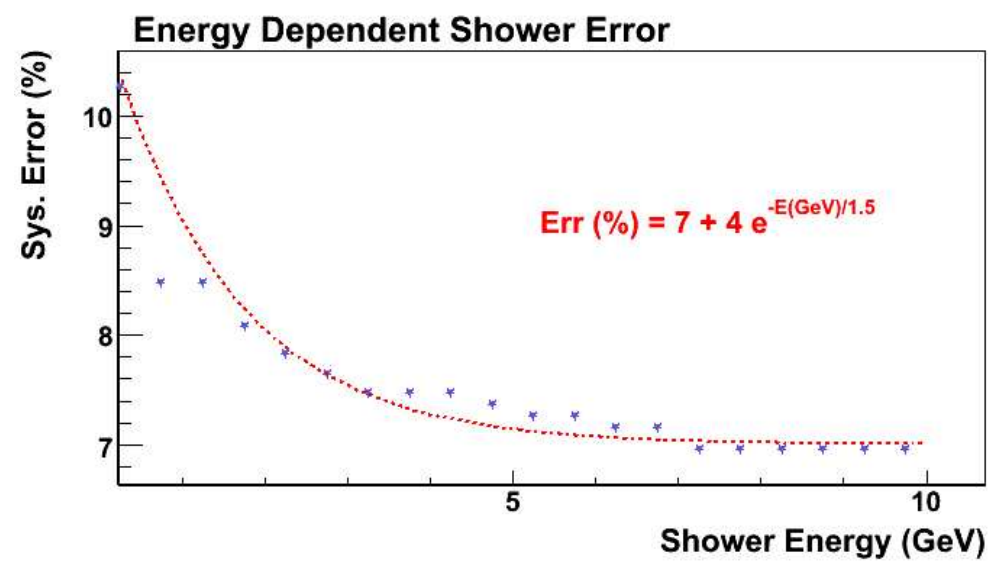

Figure 8.1: The absolute hadronic energy uncertainty as a function of shower energy estimated as discussed in the text (blue dots), and the parameterisation used in estimating the effect of the uncertainty on the antineutrino analysis (red line). Figure provided by [179].

\subsection{Muon Energy Measurement}

If a muon stops in the detector, its energy is measured from range. Comparisons of muons in the calibration detector with those in the Monte Carlo [180] showed a 3-4\% underestimate of muon range in the Monte Carlo. It is now suspected that some of this discrepancy was caused by uncertainties in the muon beam spectrum (in the energies of the muons). The uncertainty in the modelling of muon range is therefore expected to comprise a $2 \%$ uncertainty on the measured energy (identically in the near and far detectors).

Energies of muons which exit the detector are measured from their curvature in the magnetic field. The accuracy of these energy measurements is estimated through a comparison of the energy of stopping muons measured by curvature to that from range, along with direct estimates of the uncertainty on the magnetic field strength. See [174] for more details. This method of comparison to the range measurement means the aforementioned $2 \%$ uncertainty in energies measured by range also applies to curvature measurements. An additional 1\% uncertainty on the curvature measurements is estimated from uncertainties in the knowledge of 
the magnetic field strength (in both detectors).

The $2 \%$ range uncertainty is fully correlated in its effect on curvature and range measurements. $\mathrm{A} \pm 2 \%$ scale on all track energies, in both detectors, is therefore taken as a single systematic uncertainty. The extra $1 \%$ uncertainty on curvature is considered uncorrelated with the range uncertainty, so is treated separately. At the time of writing it is not clear how correlated this $1 \%$ is between the two detectors (as the field measurements were made with the same equipment some correlation is expected). To ensure the uncertainty is not underestimated, no correlation is assumed between the detectors: separate $\pm 1 \%$ uncertainties are considered at each detector.

\subsection{Relative Near to Far Detector Spectrum Nor- malisation}

Three factors contribute to an uncertainty in the relative normalisation of the measured energy spectra at the near and far detectors.

The exposure time of the far detector (proton-on-target counting), relative to that in the near detector, is uncertain to $1 \%$. This value was chosen to cover the spread between two independent attempts to calculate the exposure.

The fiducial mass of the far detector is uncertain to $2 \%$ (due to uncertainties in the steel and scintillator thicknesses and densities).

Scanning detector events by eye looked for evidence of an unmodelled near to far detector difference in the likelihood of the reconstruction software to fail to find a muon track. This difference was seen to be less than $3 \%$.

Adding these three uncertainties in quadrature yields a total $4 \%$ normalisation uncertainty. This uncertainty is used in both charged current $\nu_{\mu}$ and $\bar{\nu}_{\mu}$ analyses. 


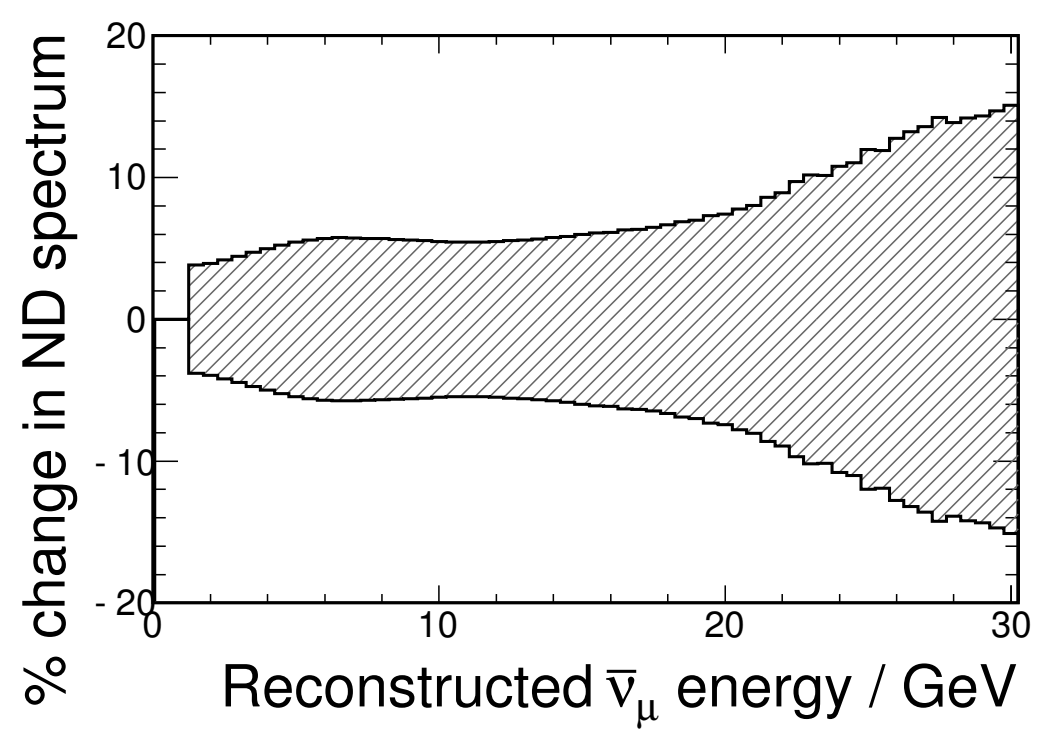

Figure 8.2: The error band on the $\bar{\nu}_{\mu}$ reconstructed energy spectrum at the near detector given by the beam reweighting parameters.

\subsection{Flux Uncertainties}

\subsubsection{General Beam Modelling}

The beam reweighting (section 3.7.1) provides a set of parameters which reweight Monte Carlo events to improve agreement between the near detector reconstructed energy spectra of data and Monte Carlo. These parameters, obtained by fitting data to Monte Carlo, are given $\pm 1 \sigma$ errors, combining to form an error band on the reconstructed energy spectra (shown in figure 8.2), correlated between the near and far detector. This error band is used as the $\pm 1 \sigma$ uncertainty on the neutrino flux. It comprises uncertainties due to overall proton-on-target counting, misalignment of the target and magnetic horn system, uncertainties in the horn currents and the fraction of the proton beam which hits the baffle. 


\subsubsection{Antineutrinos from Decay Pipe Parents}

One further uncertainty in the flux is the spectrum of antineutrinos arising from hadrons produced in the decay pipe (through hadrons produced in the target hitting the decay pipe wall, producing a further hadron which decays into an antineutrino). This effect is ignored for the neutrino beam as the number of neutrinos from hadrons produced in the target is so much higher as to render the fraction of decay-pipe originating neutrinos negligible. For antineutrinos, this decay pipe component makes up $17 \%$ of the near detector spectrum and $7 \%$ of the far detector spectrum.

The data analysed in this thesis was taken with the decay pipe under vacuum. After this data taking, it became necessary to fill the decay pipe with helium at atmospheric pressure. The upstream end of the decay pipe is sealed by a $\frac{1}{16}{ }^{-}$ inch thick aluminium window, which was found to be corroding due to the acidic environment caused by the beam. This resulted in a danger of implosion, thus the need for the helium as a safety measure.

The decay pipe component increases by around one third upon addition of the helium. Therefore a comparison of the measured and simulated near detector $\bar{\nu}_{\mu}$ spectra before and after the helium was added measures the accuracy of the decay pipe simulation. This study [181] confirmed the modelling with a sensitivity to within $42 \%$ of all antineutrinos produced from decay pipe hadrons. Therefore a uncertainty of $42 \%$ is taken as a scale factor on the number of antineutrinos produced from decay pipe parents.

Antineutrinos come from pions which are defocused by the NuMI magnetic focusing horns. These pions will be especially responsible for the antineutrino parents created in the decay pipe wall. It is possible that this defocusing field is less well modelled that the focusing field responsible for the neutrino beam. To put an upper limit on the uncertainty arising from the horn fields, near detector 

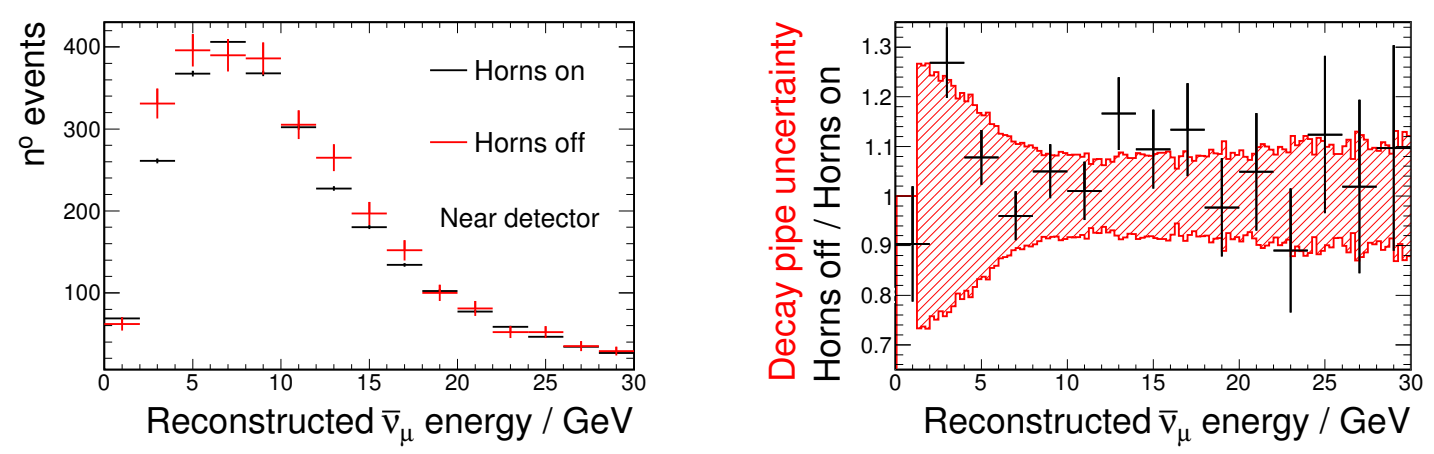

Figure 8.3: Left: the selected near detector $\bar{\nu}_{\mu}$ spectrum with the standard NuMI beam configuration (black) and with the focusing horns switched off (red). Right: the ratio of horn off to standard data (black) and an band showing the fractional effect of a $42 \%$ uncertainty on the decay pipe production (red).

data taken with the horns switched off (no field) is used. This is compared to the standard horn on data in figure 8.3 along with a band corresponding to the $42 \%$ uncertainty on the decay pipe production. The fact that the spectrum changes very little (less than 10\% across most energies) shows that the antineutrino beam is very robust against even such extreme changes in the defocusing field. Only one energy bin $(3-4 \mathrm{GeV})$ shows a larger change. This change in the focusing field is much larger than the uncertainty on that field. As the changes in all the energy bins are consistent with the red error band corresponding the the $42 \%$ decay pipe production uncertainty, this band is considered adequate to cover the total uncertainty.

An uncertainty in the geometry of the decay pipe could also produce a mismodelling of the decay pipe production. However the dimensions of the 600 metre-long iron decay pipe are known to centimetre precision, giving sub-per-cent level accuracy on this aspect of the modelling. 


\subsection{Cross Section Uncertainties}

Neutrino cross sections for the detector simulation are provided by the NEUGEN event generator [149], using the MODBYRS-4 model. Cross section uncertainties are modelled with changes in NEUGEN parameters [182]. The processes contributing to the neutrino interactions in MINOS are discussed in more detail in section 3.8.2.

At low energies (up to $\sim 1 \mathrm{GeV}$ ), quasi-elastic scattering and resonant production dominate neutrino interactions. Both of these processes are modelled in NEUGEN by a single parameter each, an axial mass, $M_{A}^{\mathrm{QE}}$ and $M_{A}^{\mathrm{Res}}$ respectively. This single-parameter modelling is not felt to well-model the data, and there are further uncertainties related to the use of the model in iron nuclei. An uncertainty on each axial mass of $15 \%$ is adequate to cover disagreements between the model and neutrino cross section data.

In the 10-30 GeV region, deep inelastic scattering (DIS) dominates neutrino interactions. Fitting to the MINOS data in this region, with input from the world average, indicated a $3 \%$ uncertainty, modelled as a scale factor on the total charged current $\nu_{\mu}+\bar{\nu}_{\mu}$ cross section.

The resonance to DIS transition region $(1-10 \mathrm{GeV})$ is modelled by a set of parameters $r_{i j k}, i=1,2$ referring to $\mathrm{CC}$, NC interactions, $j=1-4$ labelling the possible combinations of $\nu_{\mu}$ and $\bar{\nu}_{\mu}$ interacting with a proton or neutron $(1 \equiv \nu p$, $2 \equiv \nu n, 3 \equiv \bar{\nu} p, 4 \equiv \bar{\nu} n$ ), and $k$ quantifying the multiplicity of the final state. The values used for these parameters are $r_{i(1,4) 2}=0.1, r_{i(2,3) 2}=0.3$ and $r_{i j 3}=$ 1.0. Fits to data suggest uncertainties of \pm 0.1 on the $r_{i j 2}$ and \pm 0.2 on the $r_{i j 3}$ parameters [182]. The $r_{i j 3}$ parameters are bounded to be $\leq 1$, so no upwards shift is performed on this parameter.

The above are uncertainties in absolute cross sections, so are applicable to both neutrino and antineutrino interactions. However, antineutrino cross sections are 


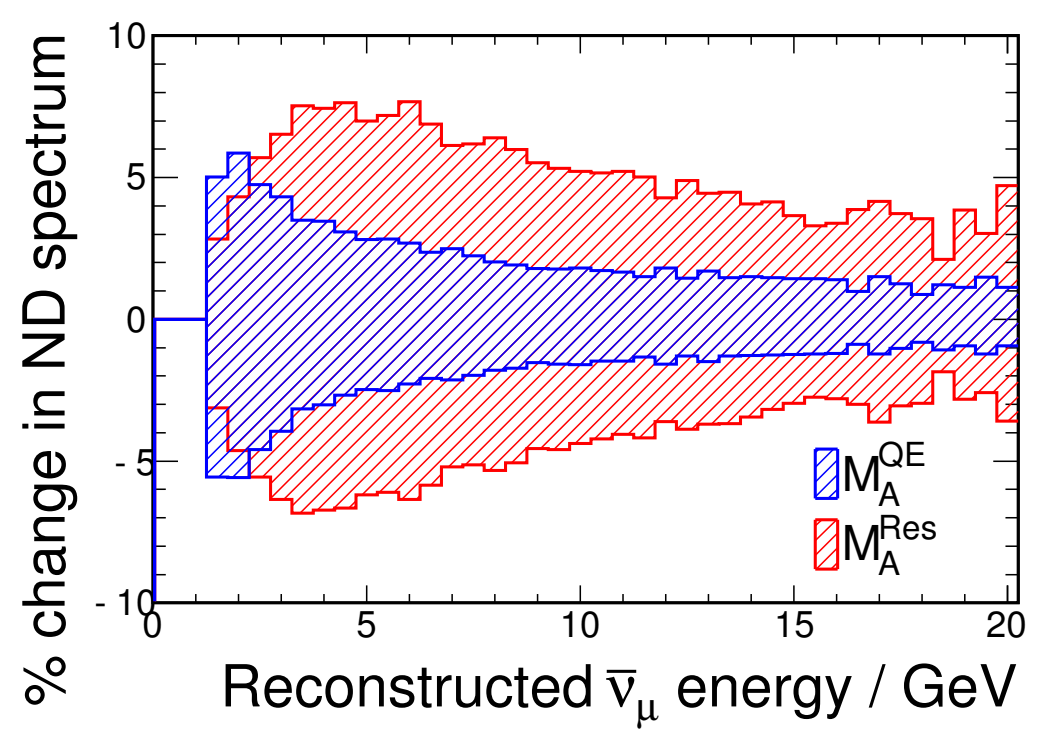

Figure 8.4: The per centage changes in the selected near detector $\bar{\nu}_{\mu}$ spectrum corresponding to the $15 \%$ systematic uncertainties in the quasi-elastic and resonance axial masses.

known most accurately from their ratio $R$ to the better measured neutrino cross sections. An additional set of uncertainties apply to antineutrino cross sections.

In the quasi-elastic and resonance region, uncertainties due to the extension of the model from free nucleons to iron nuclei are evaluated as the difference between $R$ measured for free nuclei and that measured for iron-bound nuclei: $8 \%$, modelled as scale factors on the quasi-elastic and resonance cross sections individually.

An overall uncertainty of $4 \%$ on $R$ is used to account for uncertainties in the $>10 \mathrm{GeV}$ DIS region due to QCD model uncertainties.

Fitting world data in the $1-10 \mathrm{GeV}$ region gives fit errors on the resonance to DIS transition region $\bar{\nu}_{\mu}$ parameters $r_{132}, r_{142}$ of \pm 0.2 , which are used as the systematic uncertainties for this study, with a caveat: the parameters are bounded to be greater than 0 . The default value of $r_{142}$ is 0.1 ; a -0.2 shift would take this below 0: so the parameter is set to 0 in this case.

Figures 8.4-8.6 show the effects on the selected near detector $\bar{\nu}_{\mu}$ spectrum of the systematic shifts in the charged current cross sections. 


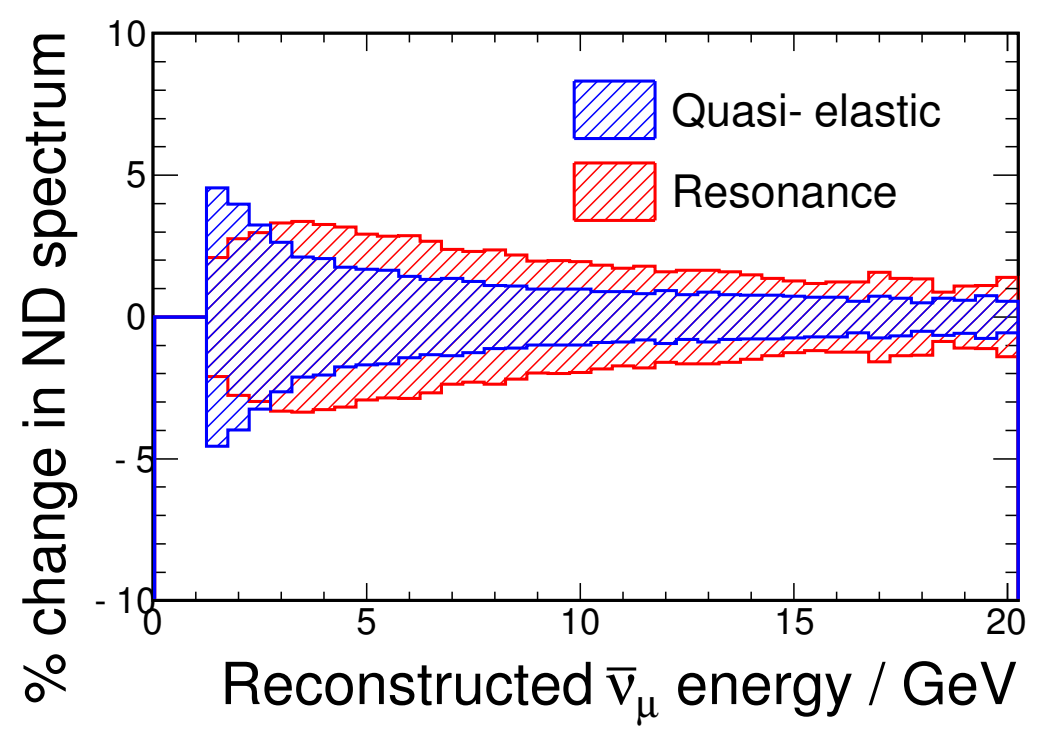

Figure 8.5: The per centage changes in the selected near detector $\bar{\nu}_{\mu}$ spectrum corresponding to the $8 \%$ systematic uncertainties in the charged current $\bar{\nu}_{\mu}$ quasielastic and resonance cross sections.

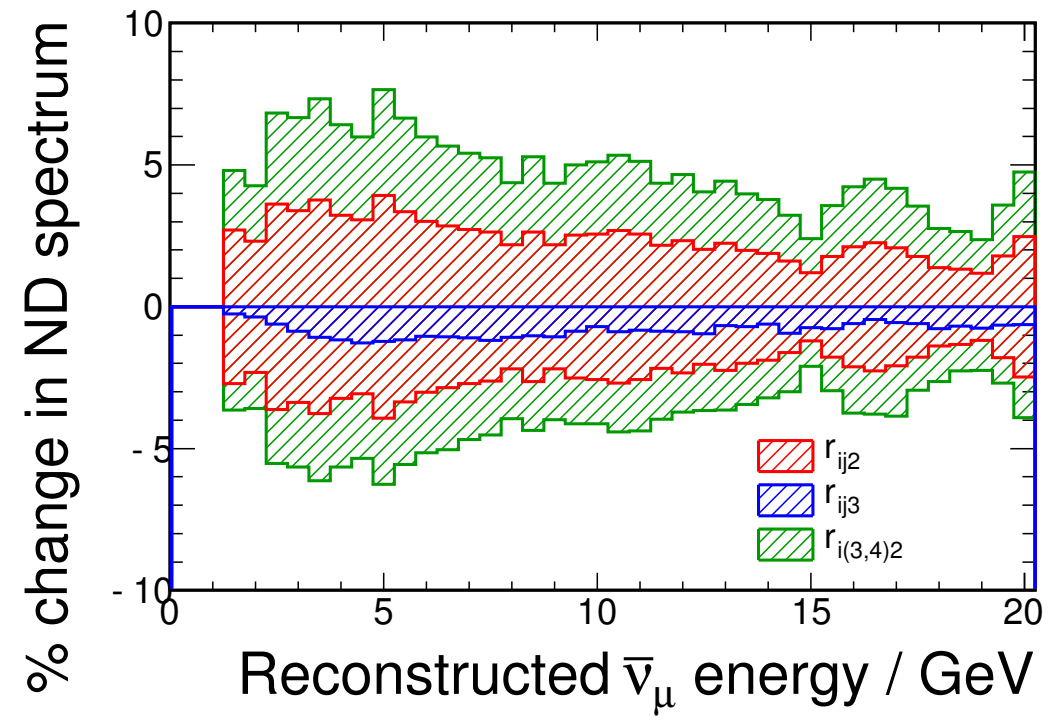

Figure 8.6: The per centage changes in the selected near detector $\bar{\nu}_{\mu}$ spectrum corresponding to the systematic uncertainties in the parameters governing the cross section in the DIS-resonance transition region. The systematic uncertainty on the $r_{i j 3}$ parameters is one-sided. 


\subsection{Neutral Current Background}

The MINOS neutrino analysis places a $50 \%$ uncertainty on the size of its neutral current background to account for neutral current hadronic shower mismodelling uncertainties. The uncertainty was estimated by removing the reconstructed muon from better understood CC events in both the data and Monte Carlo to form fake NC events [183]. This allowed more direct comparison of hadronic showers in the data and Monte Carlo.

Although this uncertainty was not estimated for the specific selection used in this analysis, the same value of $50 \%$ will be used. The selection cut requiring at least 35 planes in any track should take the selection out of the region where hadronic shower modelling can severely effect background estimation; furthermore, the selection retains so little NC background contamination (see figure 6.7) that even a larger uncertainty will not significantly affect the overall selected spectral shape.

\subsection{Charge Selection}

The charge of the muon track in an event is determined through its direction of curvature in the magnetic field. Three sources of uncertainty contribute to errors in charge identification: scattering, detector alignment and magnetic field uncertainties. An uncertainty in the magnetic field strength does not, in itself, produce a charge selection uncertainty (as the track still bends in the same direction, just by a different amount); but this uncertainty could take the level of curvature near a threshold at which multiple scattering or alignment effects could change the reconstructed direction of curvature. 


\subsubsection{Multiple Scattering}

An expression for the RMS angle of deflection (in a plane) of a charged particle undergoing Coulomb scattering is given in [58]:

$$
\theta_{0}=\theta_{\text {plane }}^{\text {rms }}=\frac{13.6 \mathrm{MeV}}{\beta c p} z \sqrt{\frac{x}{X_{0}}}\left[1+0.038 \ln \left(\frac{x}{X_{0}}\right)\right] .
$$

$\beta c$ and $p$ are the velocity and momentum of the particle, $z$ its charge in units of the electron charge, $x$ the distance travelled through the scattering medium and $X_{0}$ the radiation length of the medium.

Using the expressions for $X_{0}$ given in [58], for iron with $A=56 \mathrm{gmol}^{-1}, Z=26$ and $\rho=(7.85 \pm 0.03) \mathrm{gmol}^{-1}[140]$, one obtains $X_{0}=1.768 \mathrm{~cm}$.

This curvature due to scattering competes with that due to the magnetic field. The radius of curvature of a charged particle in such a field is given by

$$
r=\frac{p}{q B}
$$

where $B$ is the field strength and $q$ the charge of the particle. For a particle travelling a distance $x$, this corresponds to an angle of deflection

$$
\theta_{B}=\frac{x}{r}=\frac{x q B}{p}
$$

In figure 8.7 the bending angles $\theta_{B}$ and $\theta_{0}$ are compared for a muon passing through a single detector plane of steel $(2.54 \mathrm{~cm})$ perpendicular to a magnetic field of $1.3 \mathrm{~T}$ (the field strength in the saturated regions of the detectors). The muon energy has been assumed constant through the plane (the typical energy loss in a plane is $30 \mathrm{MeV}$ compared to the typical muon energies of $\mathcal{O}(1 \mathrm{GeV})$ or higher). The magnetic field causes more bending than RMS multiple scattering deviation at all energies. 


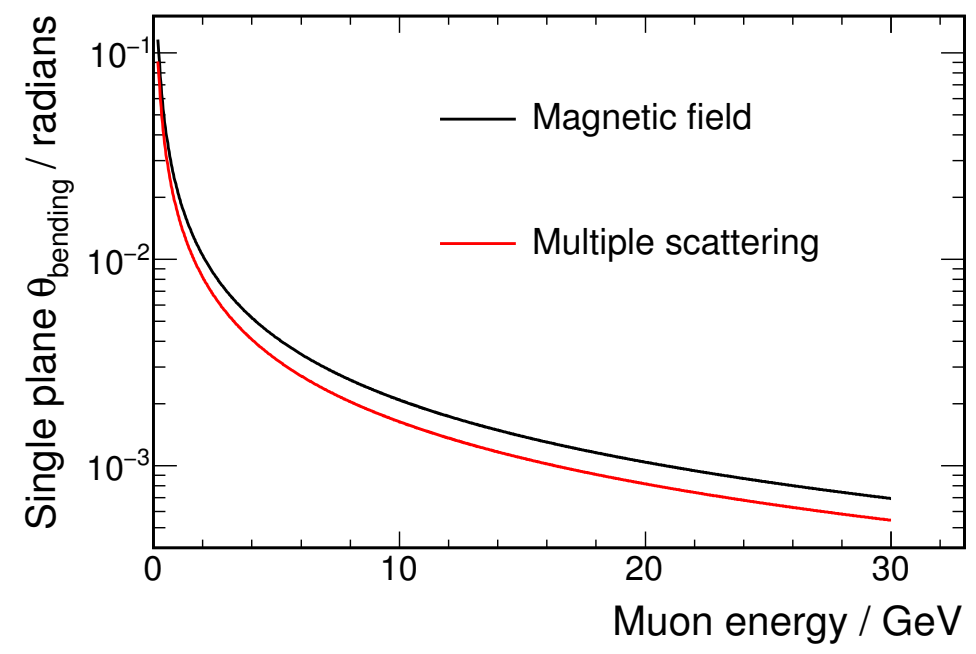

Figure 8.7: Black: the angle of bending induced in a single MINOS iron plane by the magnetic field, as a function of muon energy. Red: the corresponding RMS angle of multiple scattering.

In figure 8.8 the bending due to magnetic field and multiple scattering are compared for entire tracks. For the magnetic field, $\theta_{B}$ for each plane on the track (as calculated above) was summed, assuming constant muon energy through each plane and the muon having $30 \mathrm{MeV}$ less energy at each successive plane. $\theta_{0}$ was treated similarly, but summed in quadrature over successive planes as the curvature from multiple scattering is uncorrelated between planes. The bending is calculated assuming the tracks exit the detector after a certain number of planes (assuming the tracks have adequate initial energy to pass through this distance). A range of such maximum track lengths are calculated starting with 35 planes (the shortest tracks accepted by the CC $\bar{\nu}_{\mu}$ selection), going up to 500 planes (approximately the maximum possible track length in the far detector). It is this exiting which causes the discontinuity in the gradients in figure 8.8 (most visible in the 500 plane line): the discontinuity occurs when the track has enough energy to exit the detector. For tracks which do not exit, the final two planes have been neglected from the calculation: this prevents singularities in both bending formulae as $p \rightarrow 0$, and the assumption of constant energy loss is less applicable 


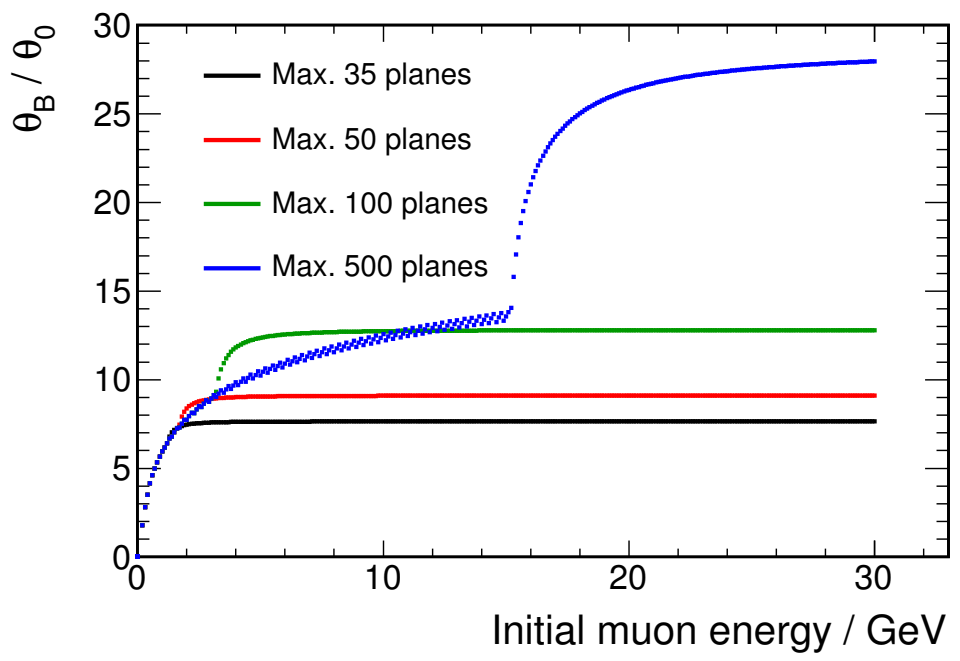

Figure 8.8: The ratio of the angle of bending due to magnetic field to the RMS angle of multiple scattering, for a muon track which exits the detector after a certain number of planes (see legend), as a function of the muon energy at the start of the track.

in these final planes.

The uncertainty on the magnetic field strength in MINOS is measured with two sources [140]. Magnetic induction coils on each detector plane measure the field strength to an accuracy of $2 \%$. Comparisons of muon energy measurement from range and curvature furthermore confirm the field strength to be modelled in the Monte Carlo to within 2\%. Furthermore, studies of muons in the calibration detector [184] showed the RMS deviation due to scattering to agree between data and simulation to within $2 \%$. The shortest accepted track length of 35 planes corresponds to an initial muon energy of $\sim 1 \mathrm{GeV}$. Figure 8.8 therefore shows that for all tracks included in the antineutrino analysis, bending from the magnetic field dominates that from multiple scattering by a factor of at least 5, up to almost 30 for longer, higher energy tracks. This is much greater than the magnetic field or scattering uncertainty, providing confidence that the selection is robust against mismodellings of the field or scattering in the Monte Carlo. 


\subsubsection{Alignment of Detector Planes}

The alignment of the detector planes is discussed in detail in [140]. A commercial optical measurement system was used during construction to ascertain the positions of the steel planes and scintillator modules to within $4 \mathrm{~mm}$ in the beam direction and $3 \mathrm{~mm}$ in the plane perpendicular to the beam. This system also kept any rotation of the scintillator modules to below $1 \mathrm{mrad}$ (corresponding to $<3 \mathrm{~mm}$ spacial offset in the ends of the scintillator strips) [185]. After installation, cosmic muons were used to confirm the alignment to within $1 \mathrm{~mm}[186,187]$.

Detector misalignment only becomes an important effect when the displacement that must be measured to determine curvature becomes comparable to the misalignment. This displacement, $d$, is defined as follows:

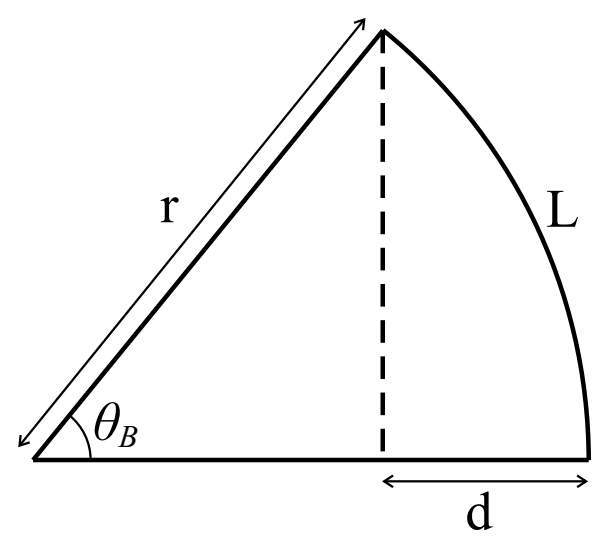

with $L$ the distance travelled by the muon through the magnetic medium (iron), and $\theta_{B}$ and $r$ the angle and radius of curvature induced by the magnetic field.

For a $30 \mathrm{GeV}$ muon which travels the full length of the far detector $L=$ $716.28 \mathrm{~cm}, r=7614.3 \mathrm{~cm}$ and $\theta_{B}=0.0941 \mathrm{rad}$ (assuming a magnetic field strength of $1.3 \mathrm{~T}$ ). This gives a displacement

$$
\begin{aligned}
d & =r\left(1-\cos \theta_{B}\right) \\
& =33.67 \mathrm{~cm} .
\end{aligned}
$$


A similar calculation for the $1 \mathrm{GeV}$ muon of the previous section yields $d=$ $30.0 \mathrm{~cm}$. These displacements are much greater than the few-millimetre alignment uncertainties, and indeed than the $4 \mathrm{~cm}$ scintillator strip width, confirming the analysis to be robust to such systematic errors.

To limit any sensitivity to muons of energies greater than $30 \mathrm{GeV}$ which will be even straighter and therefore more prone to alignment errors, no events with reconstructed neutrino energies above $30 \mathrm{GeV}$ will be used in the antineutrino analyses.

\subsubsection{Unforeseen Charge Selection Uncertainties}

The scattering model of equation 8.2 is stated to be accurate to at least $11 \%$, but to apply to only the central $98 \%$ of the distribution of scattering angles. This is because the scattering is roughly Gaussian at small angles, but has larger tails. Any mismodelling of these tails could produce an error in the charged current $\nu_{\mu}$ background estimate for this selection. To account for this, and any other unforeseen charge selection uncertainty, an estimate of the uncertainty is made from the near detector data.

A signature of any effect which causes a particle's charge to be misidentified is an incorrect measurement of energy through curvature. A sample of tracks with well-known energy is required, which can be obtained by taking tracks which stop in the detector: these have energies known to within $2 \%$ (section 8.3 ). The majority of the $\mathrm{CC}$ background falls in the region of $<10 \mathrm{GeV}$ reconstructed neutrino energy (figure 6.7); this is also the region in which the oscillation signature is expected to occur. Therefore only tracks from events with such a reconstructed neutrino energy are considered. From these events, tracks are taken which have a measured energy from range of $<5 \mathrm{GeV}$, and which pass all the antineutrino selection cuts of chapter 6 except the cuts on $C_{M}$ and $\frac{q}{p}$. The energy of these 


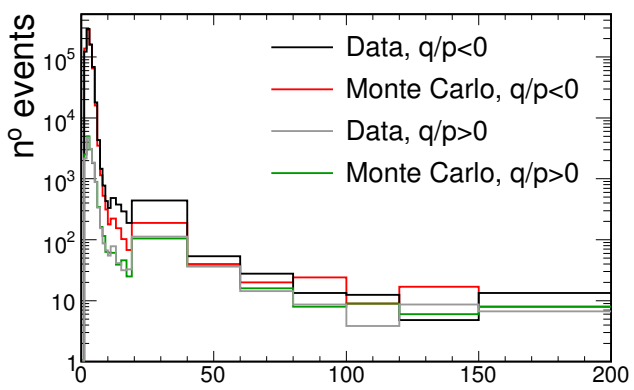

Track energy from curvature / GeV

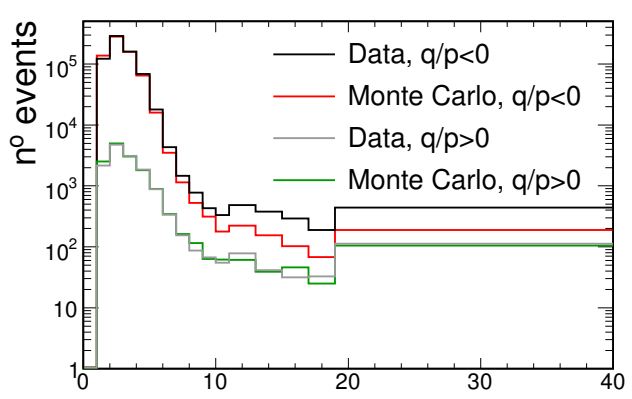

Track energy from curvature / GeV

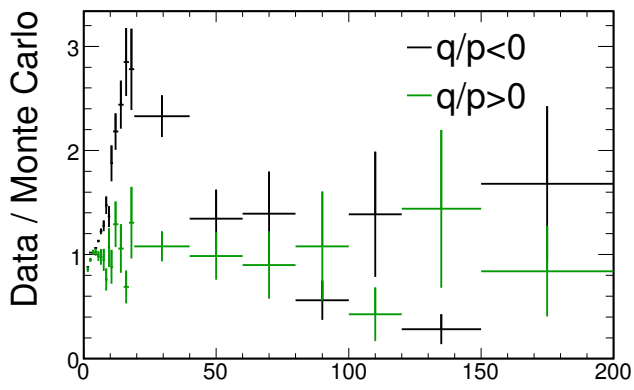

Track energy from curvature / GeV

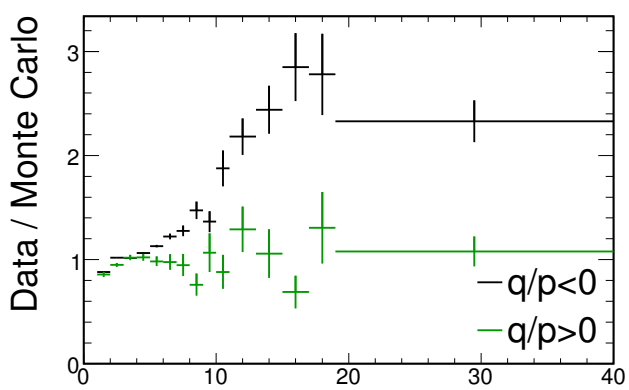

Track energy from curvature / GeV

Figure 8.9: Left: The track energy measured by curvature for contained events passing the $\bar{\nu}_{\mu}$ selection cuts (except the cuts on $\frac{q}{p}$ and $C_{M}$ ) which have a track energy measured from range of $<5 \mathrm{GeV}$ and a reconstructed neutrino energy of $<10 \mathrm{GeV}$, shown separately for tracks with $\frac{q}{p}>0$ and $\frac{q}{p}<0$. Right: the data to Monte Carlo ratio of the left hand plots. The bottom plots are identical to those at the top, but with the $x$-axis expanded.

tracks when measured from curvature is shown in figure 8.9, split into those with $\frac{q}{p}<0$ and $\frac{q}{p}>0$.

The majority of the tracks in figure 8.9 have, as expected, an energy measured from curvature of $<5 \mathrm{GeV}$; however there is a tail, stretching past $200 \mathrm{GeV}$, of tracks which have become very straight. Most of these tracks come from negative muons, thus having $\frac{q}{p}<0$; however above $\sim 50 \mathrm{GeV}$ the number in the $\frac{q}{p}>0$ and $\frac{q}{p}<0$ histograms are approximately equal, showing that for these straight tracks the reconstruction software has no sensitivity to the true charge.

The aim of figure 8.9 is to illustrate the level of agreement between data and Monte Carlo. There is a clear excess in the data for the $\frac{q}{p}<0$, at the $>100 \%$ level, showing the existence of some mismodelling in the curvature of tracks. However, 
this disagreement does not show up in the $\frac{q}{p}>0$ tracks, and therefore is not affecting the charged current $\nu_{\mu}$ background in the antineutrino selection.

The $\frac{q}{p}>0$ data to Monte Carlo ratio of figure 8.9 covers a range of approximately $0.5-1.5$. Therefore to cover the possible uncertainty allowed in the charged current $\nu_{\mu}$ background by this, a $\pm 50 \%$ scale factor is applied as a systematic uncertainty to this background, independently in each detector.

\subsection{Effect of Systematic Uncertainties on the Os- cillation Analysis}

To ascertain the effects of the various sources of systematic uncertainty on the oscillation analysis, a number of high statistics fake datasets are created from the Monte Carlo for both the near and far detectors, with oscillations applied to the far detector data. For each dataset the Monte Carlo is altered by $\pm 1 \sigma$ of each uncertainty. The extrapolation and fits are performed on each systematically shifted fake dataset. The shift in fitted parameters from the nominal (no systematic shift) case is taken as a measure of the impact of each systematic uncertainty on the analysis.

This study is performed with the neutrinos in the fake data oscillated at $\Delta m_{\mathrm{atm}}^{2}=2.43 \times 10^{-3} \mathrm{eV}^{2}$ and $\sin ^{2}\left(2 \theta_{23}\right)=1.0$ in all cases (the current MINOS best fit value [188]). Two sets of antineutrino oscillation parameters are studied: $\Delta \bar{m}_{\mathrm{atm}}^{2}=2.5 \times 10^{-3} \mathrm{eV}^{2}$ (chosen to be near to the value expected in the case of $\mathcal{C P} \mathcal{T}$ conservation) and $\Delta \bar{m}_{\mathrm{atm}}^{2}=6.0 \times 10^{-3} \mathrm{eV}^{2}$ (chosen to be in the region of MINOS's highest sensitivity to $\left.\Delta \bar{m}_{\text {atm }}^{2}\right) \cdot \sin ^{2}\left(2 \bar{\theta}_{23}\right)=1.0$ is used in both cases (as this is the expected value in the case of $\mathcal{C P} \mathcal{T}$ conservation, and also the value at which this analysis is most sensitive).

When fitting, no parameters are constrained to be in the physical region: this 


\begin{tabular}{|l|r|r|r|}
\hline \multicolumn{1}{|c|}{ Systematic } & Shift & $\delta\left(\Delta \bar{m}_{\text {atm }}^{2}\right) / 10^{-3} \mathrm{eV}^{2}$ & $\delta\left(\sin ^{2}\left(2 \bar{\theta}_{23}\right)\right)$ \\
\hline \hline Relative & $-4 \%$ & +0.46244 & -0.13821 \\
normalisation & $+4 \%$ & -0.3818 & +0.17131 \\
\hline Absolute hadronic & $-1 \sigma$ & -0.06236 & +0.0181 \\
energy measurement & $+1 \sigma$ & +0.05159 & -0.01013 \\
\hline Far detector & $-2.4 \%$ & -0.06542 & +0.0209 \\
hadronic energy & $+2.4 \%$ & +0.07797 & -0.023783 \\
\hline Near detector & $-2.3 \%$ & +0.05637 & -0.018026 \\
hadronic energy & $+2.3 \%$ & -0.05757 & +0.0195 \\
\hline Beam & $-1 \sigma$ & +0.01183 & -0.004 \\
modelling & $+1 \sigma$ & -0.01068 & +0.00364 \\
\hline Decay pipe & $-42 \%$ & -0.39953 & +0.19087 \\
production & $+42 \%$ & +0.29236 & -0.094595 \\
\hline Muon energy & $-2 \%$ & +0.02982 & -0.041541 \\
from range & $+2 \%$ & -0.01682 & +0.03461 \\
\hline Far det. muon & $-1 \%$ & -0.08311 & +0.02653 \\
energy (curvature) & $+1 \%$ & +0.10512 & -0.032065 \\
\hline Near det. muon & $-1 \%$ & +0.07582 & -0.023166 \\
energy (curvature) & $+1 \%$ & -0.07735 & +0.02584 \\
\hline Far det. wrong-sign & $-50 \%$ & +0.02296 & -0.00189 \\
CC background & $+50 \%$ & -0.02347 & +0.00228 \\
\hline Near det. wrong-sign & $-50 \%$ & -0.07711 & +0.02345 \\
CC background & $+50 \%$ & +0.07448 & -0.01997 \\
\hline NC background & $-50 \%$ & -0.01116 & +0.00845 \\
& $+50 \%$ & +0.01307 & -0.00943 \\
\hline
\end{tabular}

Table 8.1: The shifts in the measured antineutrino oscillation parameters induced by sources of systematic uncertainty at the estimated $1 \sigma$ level, at input oscillation parameters of $\Delta \bar{m}_{\mathrm{atm}}^{2}=2.5 \times 10^{-3} \mathrm{eV}^{2}, \sin ^{2}\left(2 \bar{\theta}_{23}\right)=1.0$.

could reduce any induced parameter shift, making the analysis appear unrepresentatively robust.

For the fake data oscillated at $\Delta \bar{m}_{\text {atm }}^{2}=2.5 \times 10^{-3} \mathrm{eV}^{2}$ the systematic shifts in the oscillation parameters are listed in tables 8.1 and 8.2; those for the fake data oscillated at $\Delta \bar{m}_{\text {atm }}^{2}=6.0 \times 10^{-3} \mathrm{eV}^{2}$ are listed in tables 8.3 and 8.4. The shifts are shown graphically in figures 8.10 and 8.11 for the $\Delta \bar{m}_{\text {atm }}^{2}=2.5 \times 10^{-3} \mathrm{eV}^{2}$ and $\Delta \bar{m}_{\text {atm }}^{2}=6.0 \times 10^{-3} \mathrm{eV}^{2}$ cases respectively (here all the shifts from cross-section related systematics are summed in quadrature to give a single shift).

The systematic uncertainties can be seen to be more important at the lower 


\begin{tabular}{|l|r|r|r|}
\hline \multicolumn{1}{|c|}{ Systematic } & Shift & $\delta\left(\Delta \bar{m}_{\text {atm }}^{2}\right) / 10^{-3} \mathrm{eV}^{2}$ & $\delta\left(\sin ^{2}\left(2 \bar{\theta}_{23}\right)\right)$ \\
\hline \hline$M_{A}^{\mathrm{QE}}$ & $-15 \%$ & -0.01195 & +0.00471 \\
& $+15 \%$ & +0.00602 & -0.00303 \\
\hline$M_{A}^{\text {Res }}$ & $-15 \%$ & -0.00093 & +0.00319 \\
& $+15 \%$ & -0.01641 & +0.00335 \\
\hline$r_{i j 2}$ & -0.1 & +0.01203 & -0.00401 \\
& +0.1 & -0.01131 & +0.00384 \\
\hline$r_{i j 3}$ & -0.2 & -0.00054 & +0.00117 \\
& +0.2 & 0 & 0 \\
\hline Total CC $\nu_{\mu}+\bar{\nu}_{\mu}$ & $-3.5 \%$ & -0.0008 & -0.00065 \\
cross section & $+3.5 \%$ & +0.0026 & +0.00054 \\
\hline $\bar{\nu}_{\mu}$ quasi-elastic & $-8 \%$ & -0.00458 & +0.00152 \\
cross section & $+8 \%$ & +0.00429 & -0.00139 \\
\hline $\bar{\nu}_{\mu}$ resonance & $-8 \%$ & -0.00563 & +0.00416 \\
cross section & $+8 \%$ & +0.00548 & -0.004 \\
\hline$r_{1(3,4) 2}$ & -0.2 & +0.01656 & -0.0048 \\
& +0.2 & -0.02917 & +0.00872 \\
\hline Total CC $\bar{\nu}_{\mu}$ & $-4 \%$ & +0.00566 & -0.00192 \\
cross section & $+4 \%$ & -0.00514 & +0.00174 \\
\hline
\end{tabular}

Table 8.2: As table 8.1.

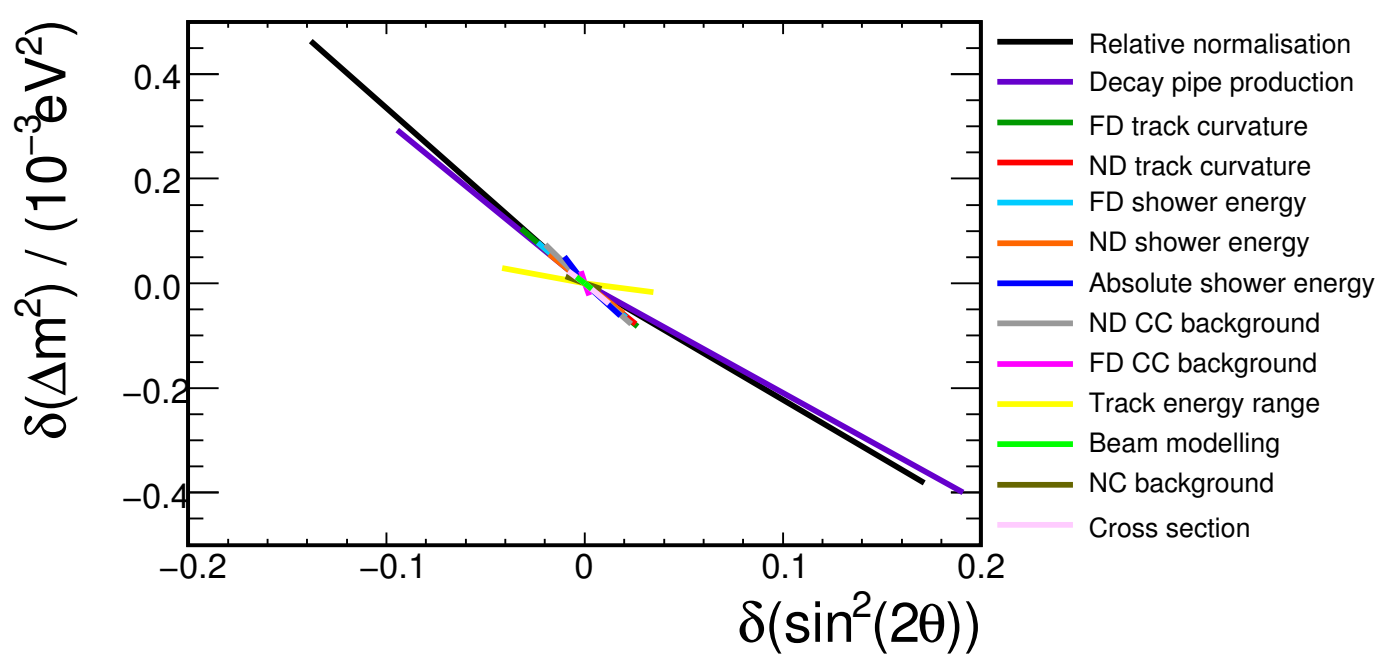

Figure 8.10: The systematic shifts of tables 8.1 and 8.2 (input oscillation parameters of $\left.\Delta \bar{m}_{\mathrm{atm}}^{2}=2.5 \times 10^{-3} \mathrm{eV}^{2}, \sin ^{2}\left(2 \bar{\theta}_{23}\right)=1.0\right)$. 


\begin{tabular}{|l|r|r|r|}
\hline \multicolumn{1}{|c|}{ Systematic } & Shift & $\delta\left(\Delta \bar{m}_{\text {atm }}^{2}\right) / 10^{-3} \mathrm{eV}^{2}$ & $\delta\left(\sin ^{2}\left(2 \bar{\theta}_{23}\right)\right)$ \\
\hline \hline Relative & $-4 \%$ & +0.09242 & +0.01031 \\
normalisation & $+4 \%$ & -0.08675 & -0.011729 \\
\hline Absolute hadronic & $-1 \sigma$ & -0.08143 & -0.003325 \\
energy measurement & $+1 \sigma$ & +0.08052 & +0.0018 \\
\hline Far detector & $-2.4 \%$ & -0.02259 & -0.003313 \\
hadronic energy & $+2.4 \%$ & +0.02456 & +0.00279 \\
\hline Near detector & $-2.3 \%$ & -0.00002 & +0.00186 \\
hadronic energy & $+2.3 \%$ & +0.0001 & -0.00192 \\
\hline Beam & $-1 \sigma$ & +0.00259 & -0.00149 \\
modelling & $+1 \sigma$ & -0.00223 & +0.00136 \\
\hline Decay pipe & $-42 \%$ & -0.08163 & -0.009733 \\
production & $+42 \%$ & +0.0602 & +0.00621 \\
\hline Muon energy & $-2 \%$ & -0.09507 & -0.00087 \\
from range & $+2 \%$ & +0.09598 & +0.00016 \\
\hline Far det. muon & $-1 \%$ & -0.02891 & -0.00389 \\
energy (curvature) & $+1 \%$ & +0.02978 & +0.00384 \\
\hline Near det. muon & $-1 \%$ & -0.0005 & +0.00246 \\
energy (curvature) & $+1 \%$ & -0.00023 & -0.00264 \\
\hline Far det. wrong-sign & $-50 \%$ & +0.0024 & +0.00405 \\
CC background & $+50 \%$ & -0.00236 & -0.00406 \\
\hline Near det. wrong-sign & $-50 \%$ & +0.00059 & -0.00287 \\
CC background & $+50 \%$ & -0.00032 & +0.00274 \\
\hline NC background & $-50 \%$ & -0.00257 & +0.00188 \\
& $+50 \%$ & +0.00273 & -0.00185 \\
\hline
\end{tabular}

Table 8.3: As table 8.1, for input oscillation parameters of $\Delta \bar{m}_{\mathrm{atm}}^{2}=6.0 \times 10^{-3} \mathrm{eV}^{2}$, $\sin ^{2}\left(2 \bar{\theta}_{23}\right)=1.0$. 


\begin{tabular}{|l|r|r|r|}
\hline \multicolumn{1}{|c|}{ Systematic } & Shift & $\delta\left(\Delta \bar{m}_{\text {atm }}^{2}\right) / 10^{-3} \mathrm{eV}^{2}$ & $\delta\left(\sin ^{2}\left(2 \bar{\theta}_{23}\right)\right)$ \\
\hline \hline$M_{A}^{\mathrm{QE}}$ & $-15 \%$ & -0.01041 & +0.00097 \\
& $+15 \%$ & +0.00978 & -0.00135 \\
\hline$M_{A}^{\text {Res }}$ & $-15 \%$ & -0.00573 & +0.00157 \\
& $+15 \%$ & +0.00213 & -0.00238 \\
\hline$r_{i j 2}$ & -0.1 & -0.00501 & +0.00061 \\
& +0.1 & +0.00478 & -0.00057 \\
\hline$r_{i j 3}$ & -0.2 & -0.00055 & -0.00018 \\
& +0.2 & 0 & 0 \\
\hline Total CC $\nu_{\mu}+\bar{\nu}_{\mu}$ & $-3.5 \%$ & +0.0023 & -0.00143 \\
cross section & $+3.5 \%$ & -0.00207 & +0.00134 \\
\hline $\bar{\nu}_{\mu}$ quasi-elastic & $-8 \%$ & -0.00327 & -0.00043 \\
cross section & $+8 \%$ & +0.00316 & +0.00043 \\
\hline $\bar{\nu}_{\mu}$ resonance & $-8 \%$ & -0.0034 & -0.00069 \\
cross section & $+8 \%$ & +0.00324 & +0.00065 \\
\hline$r_{1(3,4) 2}$ & -0.2 & -0.01139 & +0.00124 \\
& +0.2 & +0.0099 & -0.00163 \\
\hline Total CC $\bar{\nu}_{\mu}$ & $-4 \%$ & +0.00266 & -0.00125 \\
cross section & $+4 \%$ & -0.00242 & +0.00117 \\
\hline
\end{tabular}

Table 8.4: As table 8.1, for input oscillation parameters of $\Delta \bar{m}_{\mathrm{atm}}^{2}=6.0 \times 10^{-3} \mathrm{eV}^{2}$, $\sin ^{2}\left(2 \bar{\theta}_{23}\right)=1.0$.

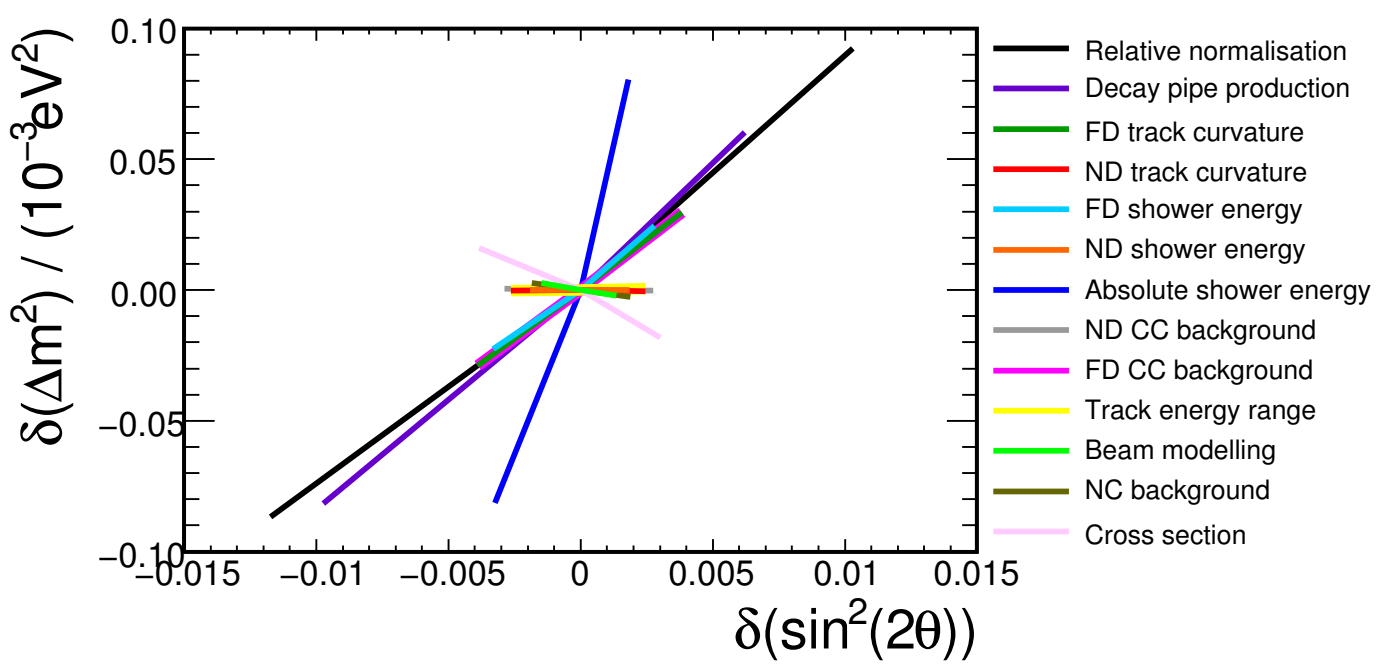

Figure 8.11: The systematic shifts of tables 8.3 and 8.4 (input oscillation parameters of $\left.\Delta \bar{m}_{\mathrm{atm}}^{2}=6.0 \times 10^{-3} \mathrm{eV}^{2}, \sin ^{2}\left(2 \bar{\theta}_{23}\right)=1.0\right)$. 
$\Delta \bar{m}_{\text {atm }}^{2}$, with the relative normalisation and decay pipe production systematics dominating, each at the level of $\sim 20 \%$ the value of both input oscillation parameters. At $\Delta \bar{m}_{\mathrm{atm}}^{2}=6.0 \times 10^{-3} \mathrm{eV}^{2}$ the dominant systematic uncertainties only contribute $1-2 \%$ the value of the input parameters, normalisation and decay pipe production still having amongst the largest effects but not dominating by as much: the absolute shower energy, in particular, now has a similar effect.

The success of the two-detector model can be clearly seen in reducing the effect of systematics which affect both detectors similarly; taking, as an example, the total CC $\bar{\nu}_{\mu}$ cross section uncertainty, compared to the relative normalisation uncertainty. Both produce a $4 \%$ scale of the observed energy spectrum, but the cross section uncertainty produces this at both detectors whilst the normalisation uncertainty produces it at only one detector. The normalisation uncertainty produces a shift of $\sim \pm 0.4 \times 10^{-3} \mathrm{eV}^{2}$ in $\Delta \bar{m}_{\mathrm{atm}}^{2}$ at a central value of $2.5 \times 10^{-3} \mathrm{eV}^{2}$, but the cross section uncertainty gives only a $\sim \pm 0.005 \times 10^{-3} \mathrm{eV}^{2}$ shift: a reduction in effect of almost three orders of magnitude as the extrapolation procedure allows the far detector prediction to be corrected for the cross section error measured by the near detector.

As the $\bar{\nu}_{\mu}$ oscillation measurement will be heavily statistically limited, these systematic uncertainties will not be included in the two-parameter contours showing the allowed regions. However, when a single-parameter error is quoted in a region near these input parameters, a systematic error will be quoted, formed from the sum in quadrature of all the parameter shifts obtained above. At $\Delta \bar{m}_{\text {atm }}^{2}=$ $2.5 \times 10^{-3} \mathrm{eV}^{2}$, this gives a $\Delta \bar{m}_{\text {atm }}^{2}$ systematic uncertainty of ${ }_{-0.582}^{+0.579} \times 10^{-3} \mathrm{eV}^{2}$ and a $\sin ^{2}\left(2 \bar{\theta}_{23}\right)$ uncertainty of ${ }_{-0.182}^{+0.265}$. At $\Delta \bar{m}_{\text {atm }}^{2}=6.0 \times 10^{-3} \mathrm{eV}^{2}$ the corresponding uncertainties are, for $\Delta \bar{m}_{\text {atm }}^{2},{ }_{-0.178}^{+0.172} \times 10^{-3} \mathrm{eV}^{2}$; and for $\sin ^{2}\left(2 \bar{\theta}_{23}\right),{ }_{-0.018}^{+0.015}$. 
8.10 Effect of Systematic Uncertainties on the $\nu_{\mu} \rightarrow \bar{\nu}_{\mu}$ Transition Analysis

\subsection{Effect of Systematic Uncertainties on the} $\nu_{\mu} \rightarrow \bar{\nu}_{\mu}$ Transition Analysis

To provide a systematic error on the level of $\bar{\nu}_{\mu}$ appearance a systematic error band on the far detector prediction is calculated. This is done at $\Delta \bar{m}_{\text {atm }}^{2}=2.5 \times$ $10^{-3} \mathrm{eV}^{2}, \sin ^{2}\left(2 \bar{\theta}_{23}\right)=1.0$ : a point near the assumed $\nu_{\mu}$ oscillation parameters $(\mathcal{C P} \mathcal{T}$ conservation is assumed for the appearance analysis).

Using the sets of systematically shifted fake data created for the oscillation systematic study above (at $\Delta \bar{m}_{\mathrm{atm}}^{2}=2.5 \times 10^{-3} \mathrm{eV}^{2}$ ), the ratio of the far detector prediction to fake data is calculated in each energy bin, for $\pm 1 \sigma$ of each systematic. Subtracted from this is the prediction to fake data ratio in the nominal (no systematic) case (otherwise any fluctuations here would counted multiple times: once for each systematic). The resulting histogram is an error band for a single systematic shift, giving the fractional prediction to fake data disagreement in each bin. The bands from each systematic shift are added in quadrature to give two overall error bands: an upper and lower band. Each bin of an individual error band is added to the total upper band if it is negative (signifying a deficit in the prediction), and to the lower band if it is positive (an excess in the prediction).

The systematic error band is shown in figure 8.12, as the fractional error on the prediction in $1 \mathrm{GeV}$ bins. Over most the spectrum $(5-20 \mathrm{GeV})$ the error remains at the 5-6\% level, dominated by the normalisation and decay pipe production systematics. Below $5 \mathrm{GeV}$ and above $20 \mathrm{GeV}$ the error increases to $10-20 \%$ as other systematics increasingly contribute. This error band will be used when discussing the results of the transition analysis in chapter 9 . 


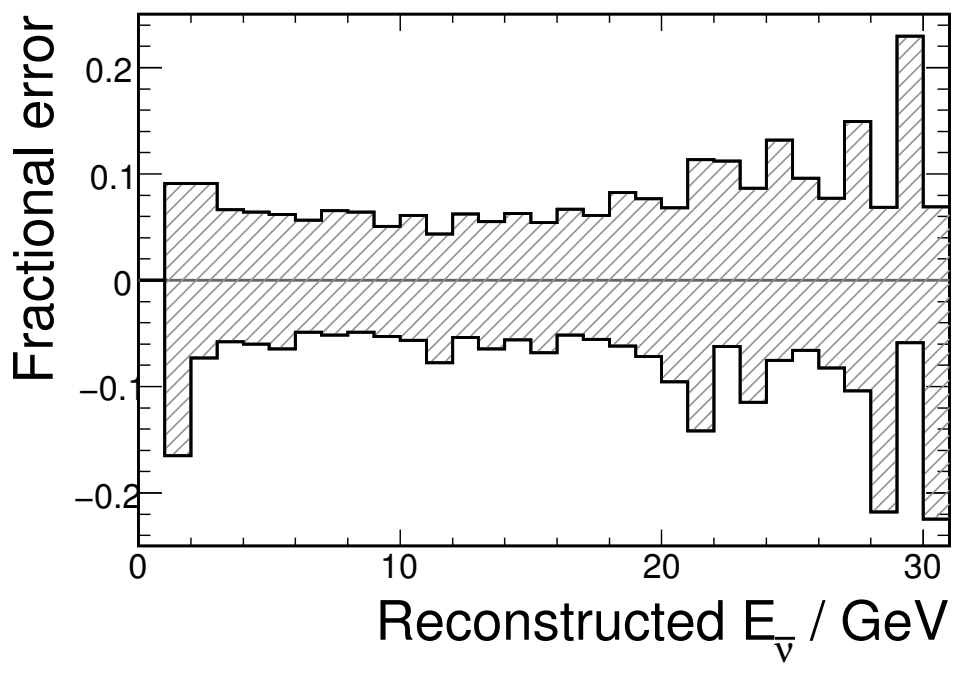

Figure 8.12: The systematic error band on the $\bar{\nu}_{\mu}$ prediction, shown as a fractional error on the predicted bin content.

\subsection{Summary}

All sources of systematic error have been discussed which are thought to affect the antineutrino analyses presented in this thesis. The effect of these sources of uncertainty on the oscillation analysis has been investigated. With true oscillation parameters of $\Delta \bar{m}_{\mathrm{atm}}^{2}=2.5 \times 10^{-3} \mathrm{eV}^{2}, \sin ^{2}\left(2 \bar{\theta}_{23}\right)=1.0$ the normalisation and decay pipe production systematics are found to dominate, giving uncertainties at the level of $\sim 20 \%$ of the parameter values. With true oscillation parameters of $\Delta \bar{m}_{\mathrm{atm}}^{2}=6.0 \times 10^{-3} \mathrm{eV}^{2}, \sin ^{2}\left(2 \bar{\theta}_{23}\right)=1.0$ the normalisation, decay pipe production and absolute shower energy systematics are the largest, giving uncertainties at the level of $1-2 \%$ the value of the oscillation parameters. A systematic error band on the far detector prediction has been calculated, which will be used in the $\nu_{\mu} \rightarrow \bar{\nu}_{\mu}$ transition analysis. 


\section{Chapter 9}

\section{Charged Current $\bar{\nu}_{\mu}$ Analysis}

\section{$9.1 \quad$ Introduction}

The far detector $\bar{\nu}_{\mu}$ data was not looked at until the rest of the analysis set out in this thesis was finalised, so as to minimise the possibility of biasing the answer towards any expected result. For the purposes of this thesis, the data has been opened to analysis independently of the rest of the collaboration. The other members of the collaboration will continue to perform extra cross checks prior to looking at the data (for example studies of multiple mock data sets, and more detailed analyses of the systematic uncertainties).

This chapter details the results of applying the antineutrino selection, extrapolation and fits developed in this thesis to the far detector data. The combination of the two low energy data taking periods gives an exposure of $3.21 \times 10^{20} \mathrm{PoT}$, the data from the two periods (of $1.27 \times 10^{20}$ PoT and $1.94 \times 10^{20}$ PoT) being separately compared to individual predictions made from the near detector data, using the statistical likelihood calculated through equation 7.16. 


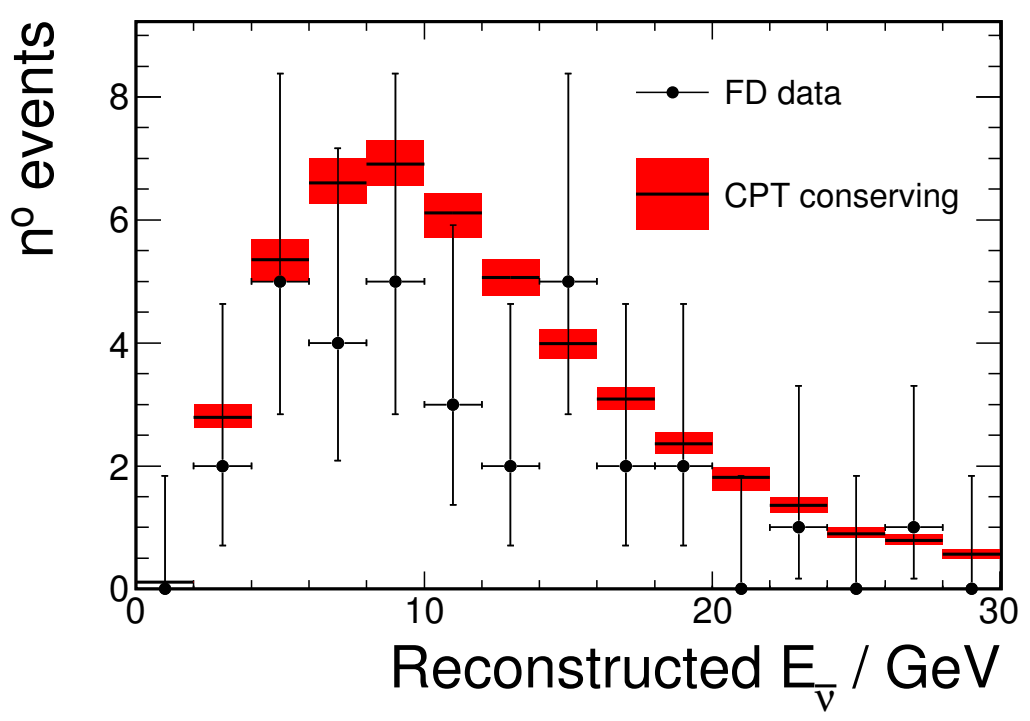

Figure 9.1: The far detector $\mathrm{CC} \bar{\nu}_{\mu}$ data (black dots) with the $\mathcal{C P} \mathcal{T}$-conserving prediction (black line) and systematic error band (red). The two data taking periods have been summed.

\subsection{Far Detector Data}

The far detector data is shown, summed over the two data taking periods, in figure 9.1, along with the prediction from the near detector data in the case of $\mathcal{C P} \mathcal{T}$-conserving oscillations $\left(\Delta \bar{m}_{\text {atm }}^{2}=2.43 \times 10^{-3} \mathrm{eV}^{2}\right)$. To the prediction has been added the systematic error band calculated in section 8.10.

For reconstructed energies below $30 \mathrm{GeV}$ (the energies used in all fits), 32 events are observed (9 in the first period, 23 in the second) whereas 47.8 are expected in the case of $\mathcal{C P} \mathcal{T}$-conserving oscillations (19.1 in the first period, 28.8 in the second). This is a statistical deficit of $2.29 \sigma(2.31 \sigma$ in the first period, $1.08 \sigma$ in the second, assuming Gaussian errors on the predicted number in all cases).

These numbers of events are shown in table 9.1, for all energies, and split into regions of $<10 \mathrm{GeV}$ and $>10 \mathrm{GeV}$ (the $<10 \mathrm{GeV}$ region being where most of the oscillation signal will occur for $\Delta \bar{m}_{\mathrm{atm}}^{2}=\Delta m_{\mathrm{atm}}^{2}$ ). A clear deficit is seen across all energies, and is at most pronounced in the region above $10 \mathrm{GeV}$, where no oscillation signal is expected. 


\begin{tabular}{|c|r|r|r|r|r|r|r|r|r|}
\hline \multirow{2}{*}{$\begin{array}{c}\text { Energy } \\
\text { region }\end{array}$} & \multicolumn{4}{|c|}{ Observed } & \multicolumn{3}{c|}{$\begin{array}{c}\text { Expected } \\
\left(\Delta \bar{m}_{\text {atm }}^{2}=2.43 \times 10^{-3} \mathrm{eV}^{2}\right)\end{array}$} & \multicolumn{3}{c|}{ Statistical deficit } \\
\cline { 2 - 9 } & Run I & Run II & Total & Run I & Run II & Total & Run I & Run II & Total \\
\hline \hline All energies & 10 & 26 & 36 & 20.2 & 30.5 & 50.8 & $2.27 \sigma$ & $0.81 \sigma$ & $2.08 \sigma$ \\
$<30 \mathrm{GeV}$ & 9 & 23 & 32 & 19.1 & 28.8 & 47.8 & $2.31 \sigma$ & $1.08 \sigma$ & $2.29 \sigma$ \\
$<10 \mathrm{GeV}$ & 4 & 12 & 16 & 8.7 & 13.1 & 21.8 & $1.59 \sigma$ & $0.30 \sigma$ & $1.24 \sigma$ \\
$10-30 \mathrm{GeV}$ & 5 & 11 & 16 & 10.4 & 15.7 & 26.0 & $1.67 \sigma$ & $1.19 \sigma$ & $1.96 \sigma$ \\
\hline
\end{tabular}

Table 9.1: Numbers of events observed and expected in the case of $\mathcal{C P} \mathcal{T}$-conserving oscillations $\left(\Delta \bar{m}_{\text {atm }}^{2}=2.43 \times 10^{-3} \mathrm{eV}^{2}\right.$, $\left.\sin ^{2}\left(2 \bar{\theta}_{23}\right)=1.0\right)$ in the far detector CC $\bar{\nu}_{\mu}$ spectrum, along with the corresponding statistical deficit observed in the data. 


\subsection{Oscillation Analysis}

Comparing the data of figure 9.1 with the prediction in the case of no oscillations (to either the $\nu_{\mu}$ or $\bar{\nu}_{\mu}$ components) gives $\chi^{2} / N_{\text {DoF }}=29.87 / 28$ (the $\chi^{2}$ being calculated in $2 \mathrm{GeV}$ bins up to $30 \mathrm{GeV}$ ). Applying oscillations under the assumption of $\mathcal{C P} \mathcal{T}$ conservation, $\Delta \bar{m}_{\mathrm{atm}}^{2}=\Delta m_{\mathrm{atm}}^{2}=2.43 \times 10^{-3} \mathrm{eV}^{2}$ (the current MINOS $\nu_{\mu}$ best fit point [188]), improves the fit to $\chi^{2} / N_{\text {DoF }}=25.70 / 28$. Throughout the rest of this chapter, the $\nu_{\mu}$ component of the prediction will be oscillated with this value of $\Delta m_{\mathrm{atm}}^{2}$.

The $\sim 2 \sigma$ depletion observed above $10 \mathrm{GeV}$ (table 9.1) means the fast oscillation limit of equation 2.5, obtained through taking $\Delta \bar{m}_{\mathrm{atm}}^{2} \rightarrow \infty$, gives a good fit to the spectrum. Indeed, with $\Delta \bar{m}_{\text {atm }}^{2}$ set to be effectively infinite and fitting for $\sin ^{2}\left(2 \bar{\theta}_{23}\right)$ only, a best fit of $\sin ^{2}\left(2 \bar{\theta}_{23}\right)=0.835$ is obtained with $\chi^{2} / \mathrm{N}_{\text {DoF }}=$ 20.11/28 (5.59 units of $\chi^{2}$ better than the $\mathcal{C P} \mathcal{T}$ conserving case).

Searching the full range of parameter space, the best fit oscillation parameters are $\Delta \bar{m}_{\text {atm }}^{2}=106.35 \times 10^{-3} \mathrm{eV}^{2}, \sin ^{2}\left(2 \bar{\theta}_{23}\right)=0.869$, with $\chi^{2} / \mathrm{N}_{\text {DoF }}=18.76 / 28$. This is $\Delta \chi^{2}=6.94$ better than the $\mathcal{C P} \mathcal{T}$ conserving case, disfavouring $\mathcal{C P} \mathcal{T}$ conservation at $\sqrt{6.94}=2.63 \sigma$; when drawing the two-parameter confidence intervals, $\mathcal{C P} \mathcal{T}$ conservation will fall outside the $95 \%$ interval (but within the $99 \%$ interval). The prediction at this best fit point is shown in figure 9.2 (combined over the two data taking periods).

The full $\chi^{2}$ surface is shown in figure 9.3, showing several local minima. In the same figure are shown the $68 \%, 90 \%$ and $99 \%$ confidence limits, along with the current MINOS best fit for the neutrino $\Delta m_{\mathrm{atm}}^{2}[188]$ which is consistent with the antineutrino $99 \%$ c.l., but not with the $90 \%$ or $68 \%$ limits. Using a $\Delta \chi^{2}=$ 1.0 around this global minimum gives $68 \%$ c.l. measurements of the oscillation parameters of $\Delta \bar{m}_{\text {atm }}^{2}=106.4_{-10.2}^{+8.9}($ stat $) \times 10^{-3} \mathrm{eV}^{2}, \sin ^{2}\left(2 \bar{\theta}_{23}\right)=0.87_{-0.23}^{+0.20}$ (stat) (noting that the upper limit includes the physical $\sin ^{2}\left(2 \bar{\theta}_{23}\right)$ boundary). 


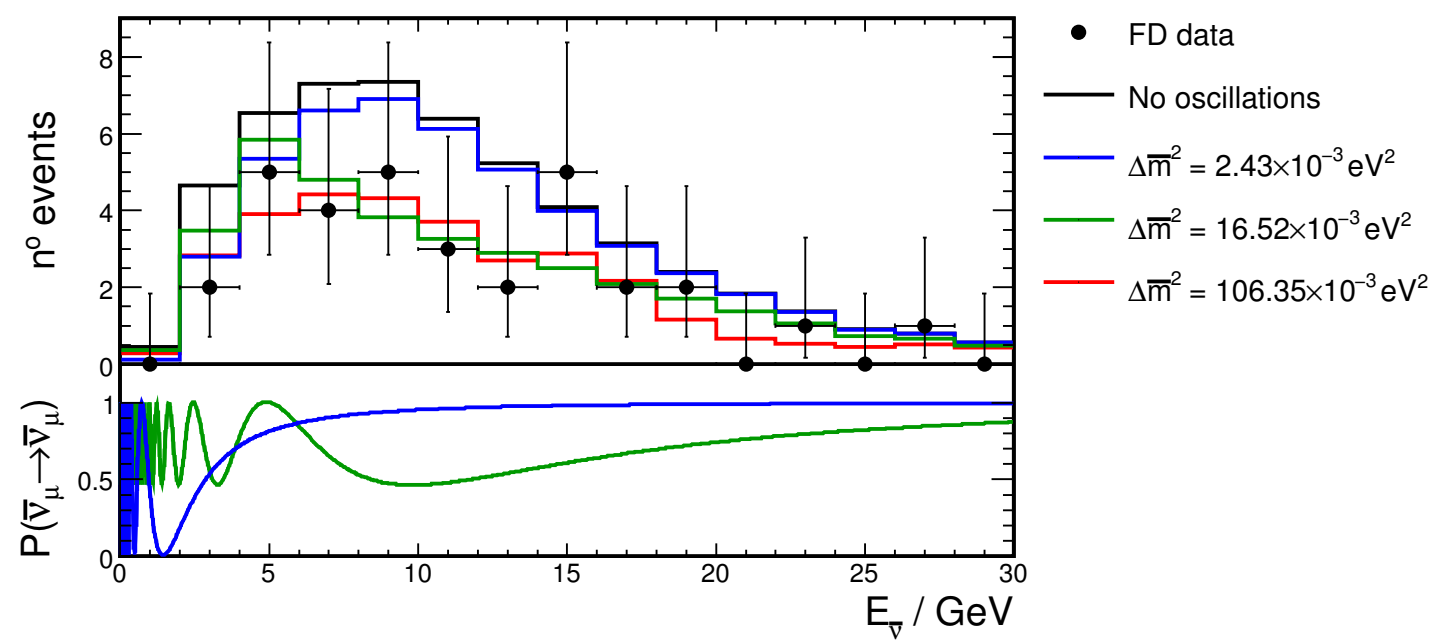

Figure 9.2: The reconstructed energy spectrum of the far detector $\bar{\nu}_{\mu} \mathrm{CC}$ data (black dots), unoscillated prediction (black line) and predictions at a variety of $\Delta \bar{m}_{\text {atm }}^{2}$ values (red, blue and green). Shown below the energy spectra are the oscillation functions (in true $\bar{\nu}_{\mu}$ energy) at two of the $\Delta \bar{m}_{\mathrm{atm}}^{2}$ values (see text).

The likelihood surface shows a local minimum around the $\Delta \bar{m}_{\mathrm{atm}}^{2}=16.52 \times$ $10^{-3} \mathrm{eV}^{2}$ region, and a spur of low $\chi^{2}$ jutting out towards $\sin ^{2}\left(2 \bar{\theta}_{23}\right)=1.0$ in the region of $\Delta \bar{m}_{\mathrm{atm}}^{2} \sim 3 \times 10^{-3} \mathrm{eV}^{2}$. To show why these regions exist, figure $9.2 \mathrm{ad}-$ ditionally shows the prediction at the $\chi^{2}$ minimum at $\Delta \bar{m}_{\mathrm{atm}}^{2}=16.52 \times 10^{-3} \mathrm{eV}^{2}$, $\sin ^{2}\left(2 \bar{\theta}_{23}\right)=0.540$, and the $\mathcal{C P} \mathcal{T}$ conserving prediction $\left(\Delta \bar{m}_{\text {atm }}^{2}=2.43 \times 10^{-3} \mathrm{eV}^{2}\right.$, $\left.\sin ^{2}\left(2 \bar{\theta}_{23}\right)=1.0\right)$ (which falls in the spur). The low $\chi^{2}$ in the $\Delta \bar{m}_{\text {atm }}^{2}=16.52 \times$ $10^{-3} \mathrm{eV}^{2}$ region is seen to be driven by an oscillation maximum falling at $\sim 5 \mathrm{GeV}$, which coincides with a high bin in the data. As $\Delta \bar{m}_{\text {atm }}^{2}$ decreases, the first oscillation minimum falls in this bin, describing the data badly and receiving a correspondingly high $\chi^{2}$ (forming the lower side of the local minimum). Once $\Delta \bar{m}_{\text {atm }}^{2}$ reaches the spur in $\chi^{2}$ around the $\mathcal{C P} \mathcal{T}$ conserving region, this minimum has moved past the $4-6 \mathrm{GeV}$ bin, once again modelling the data well. As this spur is highly consistent with the $\nu_{\mu}$ oscillation parameters, it may be showing sensitivity to the true $\bar{\nu}_{\mu}$ parameters. 

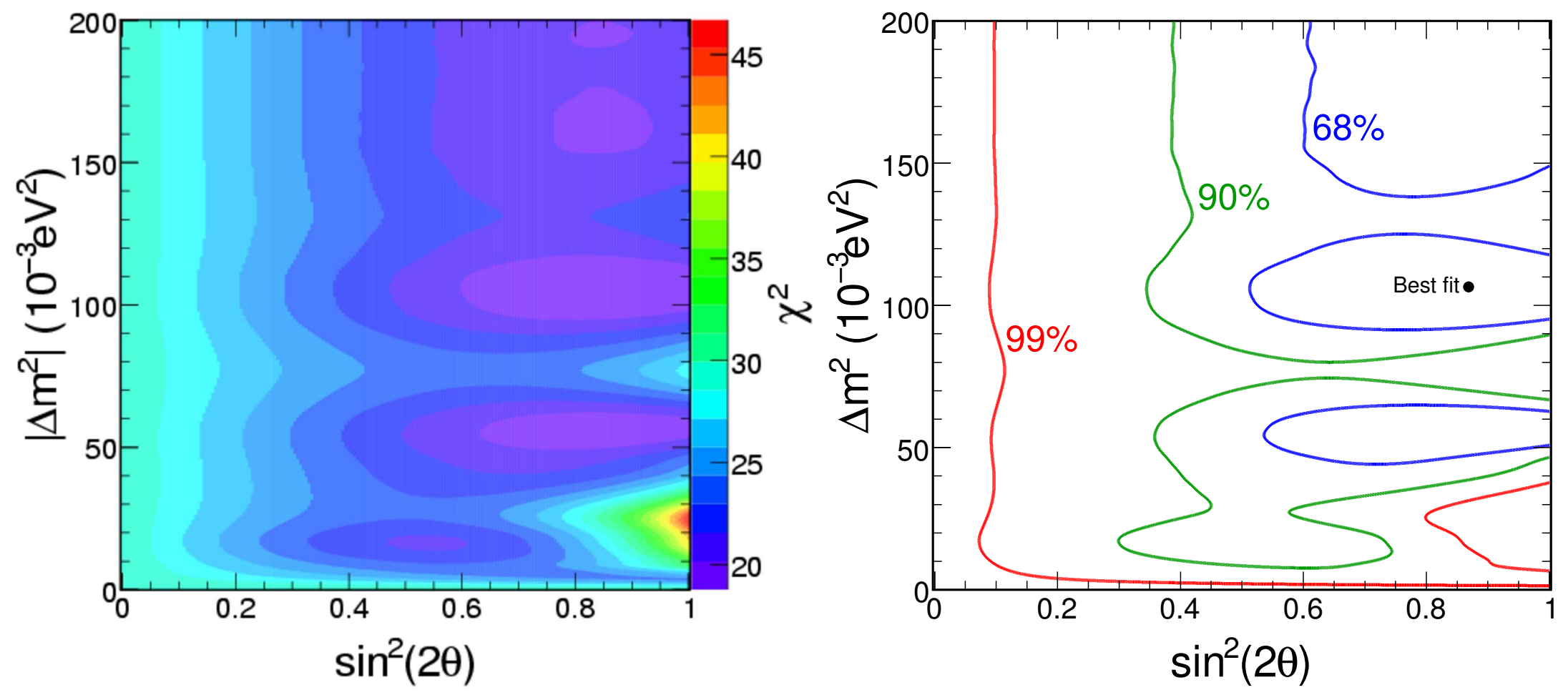

Figure 9.3: Left: the full $\chi^{2}$ surface as a function of the $\bar{\nu}_{\mu}$ oscillation parameters. Right: The $68 \%, 90 \%$ and $99 \%$ confidence intervals for the $\bar{\nu}_{\mu}$ oscillation parameters, calculated relative to the global $\chi^{2}$ minimum, obtained using $2 \mathrm{GeV}$ energy bins. 


\subsubsection{Reducing the Width of the Energy Bins}

$2 \mathrm{GeV}$ bins were chosen for the oscillation analysis shown above as this ensured all the bins were reasonably populated with events. It is reasonable to ask what extra information is obtained as the bin width is reduced towards a full unbinned likelihood treatment.

The smallest bin width investigated was $0.5 \mathrm{GeV}$. The global best fit point is still in the region of $\Delta \bar{m}_{\text {atm }}^{2}=100 \times 10^{-3} \mathrm{eV}^{2}$, but the $\Delta \chi^{2}$ between this and the $\mathcal{C P} \mathcal{T}$-conserving case is reduced by 0.72 units, to $\Delta \chi^{2}=6.2$ (or $\left.2.49 \sigma\right)$. The most noticeable feature is that the local $\chi^{2}$ minimum at $\Delta \bar{m}_{\mathrm{atm}}^{2} \sim 50 \times 10^{-3} \mathrm{eV}^{2}$ (see figure 9.3) is reduced. The full set of confidence intervals calculated in $0.5 \mathrm{GeV}$ bins are shown in figure 9.4 and can be seen to show the same general structure as those for $2 \mathrm{GeV}$ bins in figure 9.3 .

The next step would be to move onto a full unbinned likelihood fit. However, the interpretation of the data in terms of neutrino oscillations is dominated by the unexpected deficit above $10 \mathrm{GeV}$, which causes the data to favour high values of $\Delta \bar{m}_{\text {atm }}^{2}$. There is little further information to be obtained from the data beyond what has already been shown, and so the possibility of an unbinned likelihood is left for future analyses as the statistics increase.

\subsection{Single Parameter Fit to $\Delta \bar{m}_{\text {atm }}^{2}$}

Since this low-statistics experiment has minimal sensitivity to the antineutrino mixing angle, a single-parameter fit to $\Delta \bar{m}_{\text {atm }}^{2}$ can be performed, holding $\sin ^{2}\left(2 \bar{\theta}_{23}\right)=$ 1.0 (the neutrino best fit value).

A scan of the $\chi^{2}$ as a function of $\Delta \bar{m}_{\text {atm }}^{2}$ (using $2 \mathrm{GeV}$ energy bins) is shown in figure $9.5\left(\right.$ with $\left.\sin ^{2}\left(2 \bar{\theta}_{23}\right)=1.0\right)$. The series of local minima at high mass splittings are still visible, but a minimum at $\Delta \bar{m}_{\mathrm{atm}}^{2}=3.68 \times 10^{-3} \mathrm{eV}^{2}, \chi^{2} / \mathrm{N}_{\mathrm{DoF}}=24.21 / 28$ is very prominent (albeit not the minimum with the lowest $\chi^{2}$ ). As this is the only 


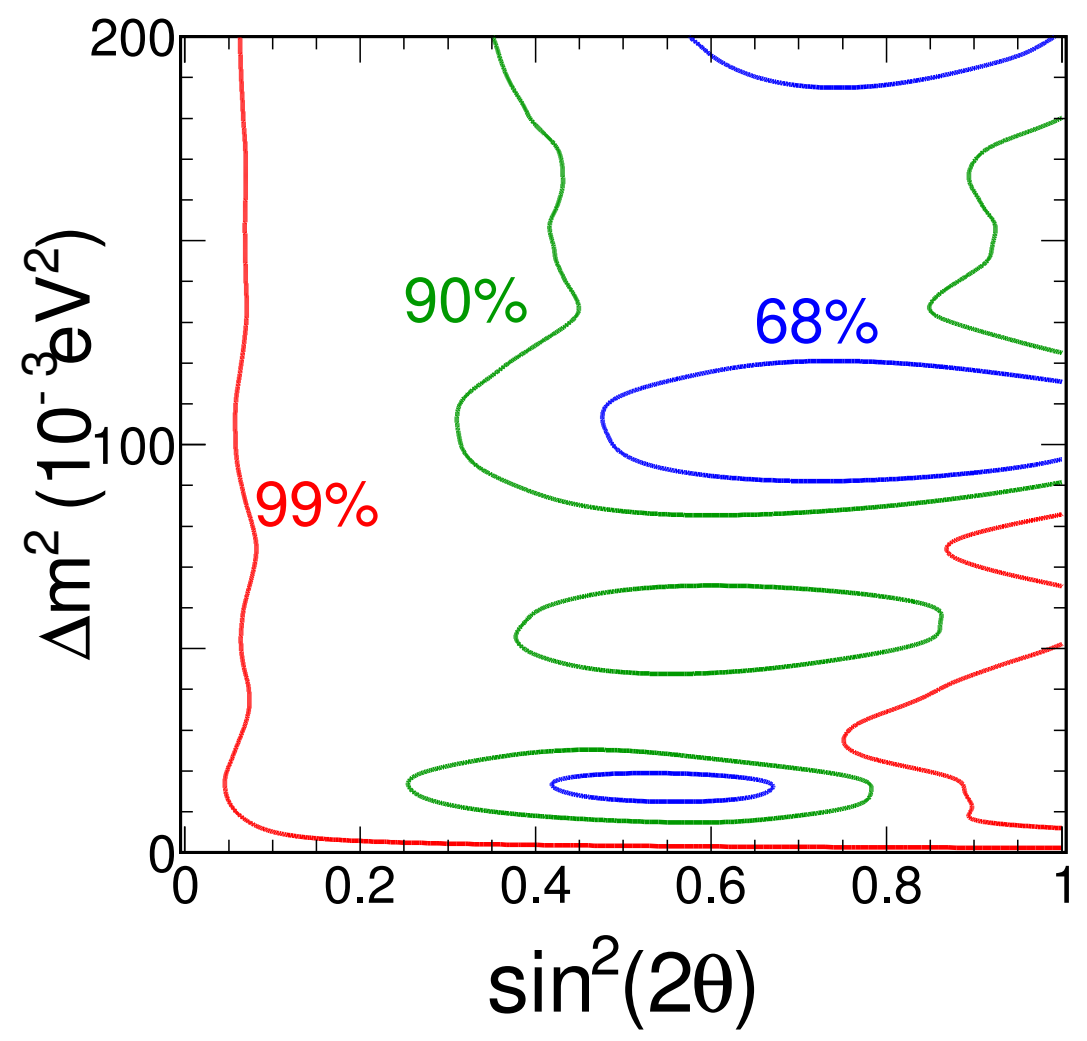

Figure 9.4: The 68\%, $90 \%$ and $99 \%$ confidence intervals for the $\bar{\nu}_{\mu}$ oscillation parameters, obtained using $0.5 \mathrm{GeV}$ energy bins.

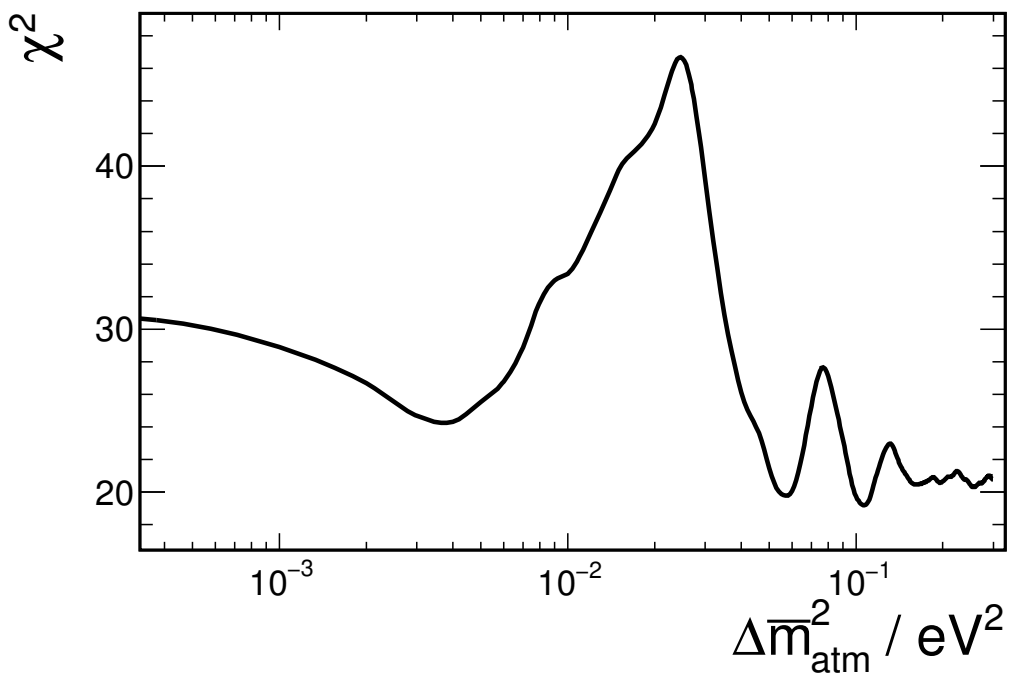

Figure 9.5: The $\chi^{2}$ as a function of $\Delta \bar{m}_{\mathrm{atm}}^{2}$ with $\sin ^{2}\left(2 \bar{\theta}_{23}\right)$ fixed to 1.0. 
minimum consistent with the world limits on $\Delta \bar{m}_{\text {atm }}^{2}$, the single parameter fit will be performed in this region. Using a $\Delta \chi^{2}=1$ and systematic errors calculated

in chapter 8 , the fit yields a $68 \%$ c.l. measurement of $\Delta \bar{m}_{\text {atm }}^{2}=3.68_{-1.00}^{+1.09}$ (stat.) \pm 0.58 (syst.) $\times 10^{-3} \mathrm{eV}^{2}$.

\section{$9.5 \bar{\nu}_{\mu}$ Appearance}

As any $\bar{\nu}_{\mu}$ appearance is expected to be the result of $\nu_{\mu} \rightarrow \bar{\nu}_{\mu}$ transitions, the appearance can only come from the $\nu_{\mu}$ that were found to have disappeared in the MINOS $\nu_{\mu}$ oscillation analysis [188]. Therefore oscillation parameters of $\Delta \bar{m}_{\mathrm{atm}}^{2}=\Delta m_{\mathrm{atm}}^{2}=2.43 \times 10^{-3} \mathrm{eV}^{2}$ are assumed. The observed $\nu_{\mu}$ disappearance occurs predominantly below energies of $10 \mathrm{GeV}$. Therefore the $\bar{\nu}_{\mu}$ appearance measurement is performed by comparing the predicted with observed numbers of events below $10 \mathrm{GeV}$, assuming these $\mathcal{C P} \mathcal{T}$ conserving oscillation parameters.

Using the systematic error band of figure 8.12, the total number of $\bar{\nu}_{\mu}$ events expected below $10 \mathrm{GeV}$ in the case of no appearance is $21.8 \pm 4.7$ (stat.) ${ }_{-1.2}^{+1.4}$ (syst.). 16 events were observed. This is a deficit of $0.98 \sigma$ (including the systematic error), and thus no evidence is observed for $\bar{\nu}_{\mu}$ appearance.

This result can be interpreted as a fraction of the $\mathrm{CC} \nu_{\mu}$ events observed to have disappeared in [188] which could have turned into antineutrinos. The number of $\nu_{\mu}$ events expected below $10 \mathrm{GeV}$ was 686.4 ; only 451 were observed. This gives 235.4 missing events. The combined selection and reconstruction efficiencies at the far detector below $10 \mathrm{GeV}$ (true neutrino energy) are $77.5 \%\left(\mathrm{CC} \nu_{\mu}\right)$ and $68.9 \%\left(\mathrm{CC} \bar{\nu}_{\mu}\right)$. The ratio of $\mathrm{CC} \nu_{\mu}$ to $\mathrm{CC} \bar{\nu}_{\mu}$ cross sections in the detector in this energy region is $\sim 2.5$. Using these as an approximate conversion factor says that $\sim 83.7$ additional $\mathrm{CC} \bar{\nu}_{\mu}$ events would be observed if the $\mathrm{CC} \nu_{\mu}$ oscillations happened purely through the $\nu_{\mu} \rightarrow \bar{\nu}_{\mu}$ channel. If a fraction $\alpha$ of the neutrinos oscillated through this channel, the additional number of selected $\mathrm{CC} \bar{\nu}_{\mu}$ events 
would therefore be $83.7 \alpha$. We measure a deficit of $5.8 \pm 4.7$ (stat.) ${ }_{-1.2}^{+1.4}$ (syst.) CC $\bar{\nu}_{\mu}$ events, which gives $\alpha=-0.069 \pm 0.056$ (stat.) ${ }_{-0.014}^{+0.017}$ (syst.).

\subsection{Summary}

The selected far detector charged current $\bar{\nu}_{\mu}$ events have been analysed in the context of oscillation and transition hypotheses. $\mathrm{A} \sim 2 \sigma$ deficit in events is seen, primarily in the 5-15 GeV region. This causes the data to slightly favour a high $\Delta \bar{m}_{\text {atm }}^{2}\left(\gtrsim 0.1 \mathrm{eV}^{2}\right)$ above other values. However, the low statistics dataset rules out very little oscillation parameter space (although compared to the global minimum no oscillations are disfavoured at the $>99 \%$ c.l.). Performing a singleparameter fit to $\Delta \bar{m}_{\text {atm }}^{2}$ in the region allowed by global limits, whilst holding $\sin ^{2}\left(2 \bar{\theta}_{23}\right)$ constant at 1.0, yields $\Delta \bar{m}_{\text {atm }}^{2}=3.68_{-1.00}^{+1.09}$ (stat.) \pm 0.58 (syst.) $\times 10^{-3} \mathrm{eV}^{2}$. Compared to the case of $\Delta \bar{m}_{\mathrm{atm}}^{2}=\Delta m_{\mathrm{atm}}^{2}$ a $0.98 \sigma$ deficit of charged current $\bar{\nu}_{\mu}$ events is observed, giving no evidence of $\nu_{\mu} \rightarrow \bar{\nu}_{\mu}$ transitions. 


\section{Chapter 10}

\section{Discussion}

\subsection{Summary}

This thesis has used data taken with the MINOS detectors from the NuMI beam to perform an analysis that has added to the current knowledge of neutrino oscillations in the atmospheric regime. This analysis has made the first use of the charged current $\bar{\nu}_{\mu}$ background in the NuMI beam to make the world's first direct measurement of $\bar{\nu}_{\mu}$ oscillations, work which will pave the way for future MINOS analyses as the amount of data increases.

Much of the work underlying this analysis has also been presented. Chapter 4 presented a method of using cosmic muons to track the response of the MINOS detectors, on a daily basis, to minimise the dependence of hadronic shower energy measurements on such time-dependent effects as detector aging and temperature changes. This formed part of a calibration chain which has allowed MINOS to come close to its original aim of measuring absolute and relative (detector-todetector) shower energies to within $5 \%$ and $2 \%$ respectively.

Chapter 5 developed two new muon charge-identification variables, and chap-

ter 6 developed these into a method of selecting antineutrino events from the NuMI beam. This selection aimed for high purity so as to minimise the potential for sys- 
tematic uncertainties, achieving near and far detector purities (in the $<10 \mathrm{GeV}$ region) of $96.5 \%$ and $98.8 \%$ respectively, the corresponding selection efficiencies being $74.4 \%$ and $70.9 \%$.

Chapter 7 explained, in detail, the beam matrix method of extrapolating a measured near detector energy spectrum to form a far detector prediction. The method was developed to allow the simultaneous extrapolation of charged current $\nu_{\mu}$ and $\bar{\nu}_{\mu}$ events with differing oscillation parameters.

Chapter 8 looked at the sources of systematic uncertainty which affect antineutrino oscillation analyses in the MINOS detectors, and showed their effect on the $\bar{\nu}_{\mu}$ oscillation measurement.

Finally, chapter 9 presented the analysis of the $\bar{\nu}_{\mu}$ data. The low statistics made this an inconclusive measurement, $\mathrm{a} \sim 2 \sigma$ deficit in the measured number of events above $10 \mathrm{GeV}$ reconstructed antineutrino energy favouring a high $\Delta \bar{m}_{\text {atm }}^{2}$ (but remaining consistent with the neutrino $\Delta m_{\text {atm }}^{2}$ at the $99 \%$ c.l.). No evidence for an appearance of charged current $\bar{\nu}_{\mu}$ events above that expected from the beam was observed.

\subsection{Antineutrino Analysis}

The measured antineutrino spectrum, shown in figure 9.1, revealed a $2 \sigma$ deficit in events in the region $10-30 \mathrm{GeV}$ : a region where negligible disappearance due to oscillations is expected. An almost identical deficit is observed at $0-10 \mathrm{GeV}$. The shape of the low-energy deficit, particularly below $6 \mathrm{GeV}$, means that a single parameter fit to $\Delta \bar{m}_{\text {atm }}^{2}$ in the region around the neutrino oscillation parameters, whilst keeping $\sin ^{2}\left(2 \bar{\theta}_{23}\right)$ fixed at 1.0, gives a measurement of $\Delta \bar{m}_{\text {atm }}^{2}=$ $3.68_{-1.00}^{+1.09}$ (stat.) \pm 0.58 (syst.) $\times 10^{-3} \mathrm{eV}^{2}$, which is consistent with the neutrino parameters. However, this leaves the higher energy region unexplained.

A candidate for the deficit is some unforeseen form of systematic uncertainty. 
The class of uncertainty which was the biggest concern before the far detector data had been observed was that of background estimation. With a large potential source of wrong-sign $\mathrm{CC} \nu_{\mu}$ background, a small uncertainty in the charge selection method could cause a sizable underestimate of the background. This was tackled through the development of the high purity selection of chapter 6 which aimed to cut so harshly on the background that the possible uncertainties would not be large enough to let significant extra background through. Additionally, chapter 8 made an estimate of the uncertainty on the amount of $\mathrm{CC} \nu_{\mu}$ background and found the uncertainty to be less than $50 \%$. This level of confidence would seem to be vindicated as such an error in background estimation would result in an excess of events in the data: not the observed deficit.

It is harder to conceive a mechanism which produces a deficit as this requires an error in the selection and reconstruction efficiency estimation. The two-detector model of MINOS aims to minimise such a possibility. Uncertainties in the beam spectrum, cross sections, and many detector effects act in the same way at both detectors such as to cancel though use of the extrapolation developed in chapter 7 . The only possibility is a factor which affects the far detector only, such as an uncertainty in the magnetic field strength or the alignment of the scintillator strips and steel planes. However, the deficit is seen across the full energy spectrum so must be affecting tracks which bend by a significant amount, as discussed in section 8.8.

To check that some specific aspect of the event selection is not responsible for the deficit, the far detector spectrum is obtained using a simple selection designed to be as independent as possible from that used for the analysis, whilst retaining comparable purity. The resultant spectrum is shown in figure 10.1 alongside a graph showing the estimated background levels. This selection uses only three cuts: two are used in the analysis selection $\left(\frac{q}{p}>0\right.$ and track length $\geq 35$ planes); the third is a cut on the neutral current discrimination variable used for the $\mathrm{CC}$ 
$\nu_{\mu}$ selection as described in section 6.5 , but at a much harsher value. ${ }^{1}$ In the region $10-30 \mathrm{GeV}$ only $63 \%$ of the expected events are observed (compared to the unoscillated far detector Monte Carlo), a similar deficit to the analysis selection for which only $60 \%$ of the expected events are observed. There is still the potential of a problem with the variable $\frac{q}{p}$ as this is common between both these selections, and is the most powerful curvature discriminant. The exercise is therefore repeated using the full analysis selection but without the $\frac{q}{p}$ cut; the resultant spectrum is also shown in figure 10.1. The selection purity is now much lower, but still only $59 \%$ of the expected events are seen in the $10-30 \mathrm{GeV}$ region suggesting any problem is not limited to $\frac{q}{p}$ (majority curvature is here providing the charge sensitivity).

Figure 10.2 shows the radial position (squared) of track vertices (the point at which the muon track is judged by the reconstruction software to have begun) for both the $\nu_{\mu}$ and $\bar{\nu}_{\mu}$ selections of chapter 6 (for the neutrino analysis, only tracks longer than 35 planes are shown, to increase the similarity to the antineutrino sample). The neutrino events show a slight drop-off in the data towards the edge of the detector beyond that which is modelled in the Monte Carlo. The low statistics of the antineutrino sample do not allow such an effect to be clearly seen; however, such an acceptance effect could be responsible for the deficit and is a good avenue for investigation as statistics increase.

As a second possibility, the deficit could be due to some $\mathcal{C P} \mathcal{T}$-violating physics effect. The deficit seen requires a large $\Delta \bar{m}_{\text {atm }}^{2}$, in the region of $0.1 \mathrm{eV}^{2}$ or higher (MINOS has no sensitivity to the detailed value of $\Delta \bar{m}_{\mathrm{atm}}^{2}$ in the region of $0.1 \mathrm{eV}^{2}$ or above). This would seem to be disfavoured by the world limits on $\Delta \bar{m}_{\text {atm }}^{2}$, discussed in section 2.13, of below $\sim 12 \mathrm{eV}^{2}$; but it is consistent with the observations of the LSND collaboration (see section 2.15) which observed $\bar{\nu}_{e}$ appearance from the channel $\bar{\nu}_{\mu} \rightarrow \bar{\nu}_{e}$ at a mass scale of the required order, with an allowed

\footnotetext{
${ }^{1}$ The cut requires the value of the $k$ nearest neighbour variable to be greater than 0.625 .
} 

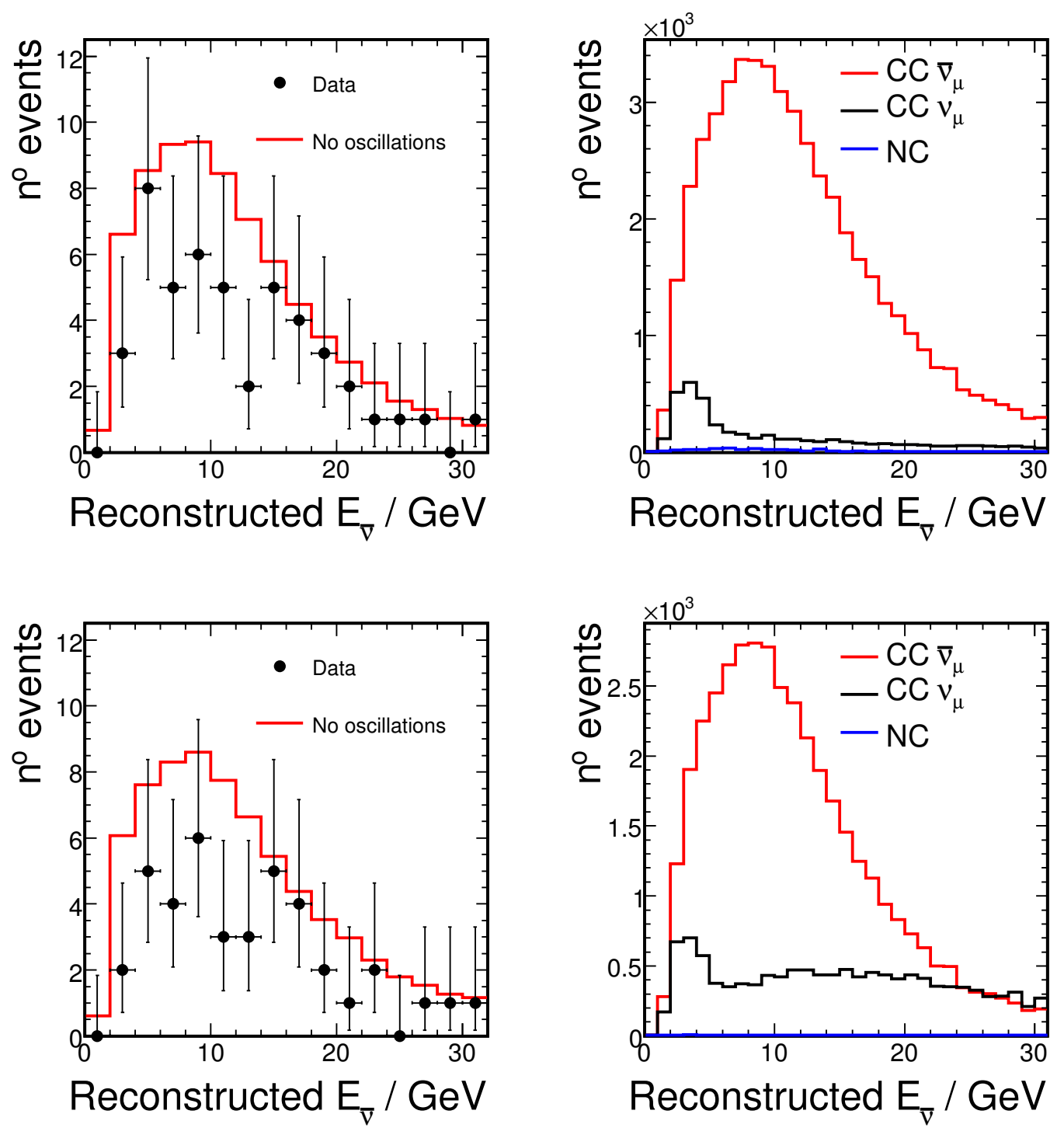

Figure 10.1: Left: the far detector data CC $\bar{\nu}_{\mu}$ spectrum, and Monte Carlo spectrum in the case of no oscillations, for two alternative selections: one based on the CC $\nu_{\mu}$ selection variable (top), and the analysis selection without the cut on $\frac{q}{p}$ (bottom). The right hand graphs show the components of the Monte Carlo spectra. 

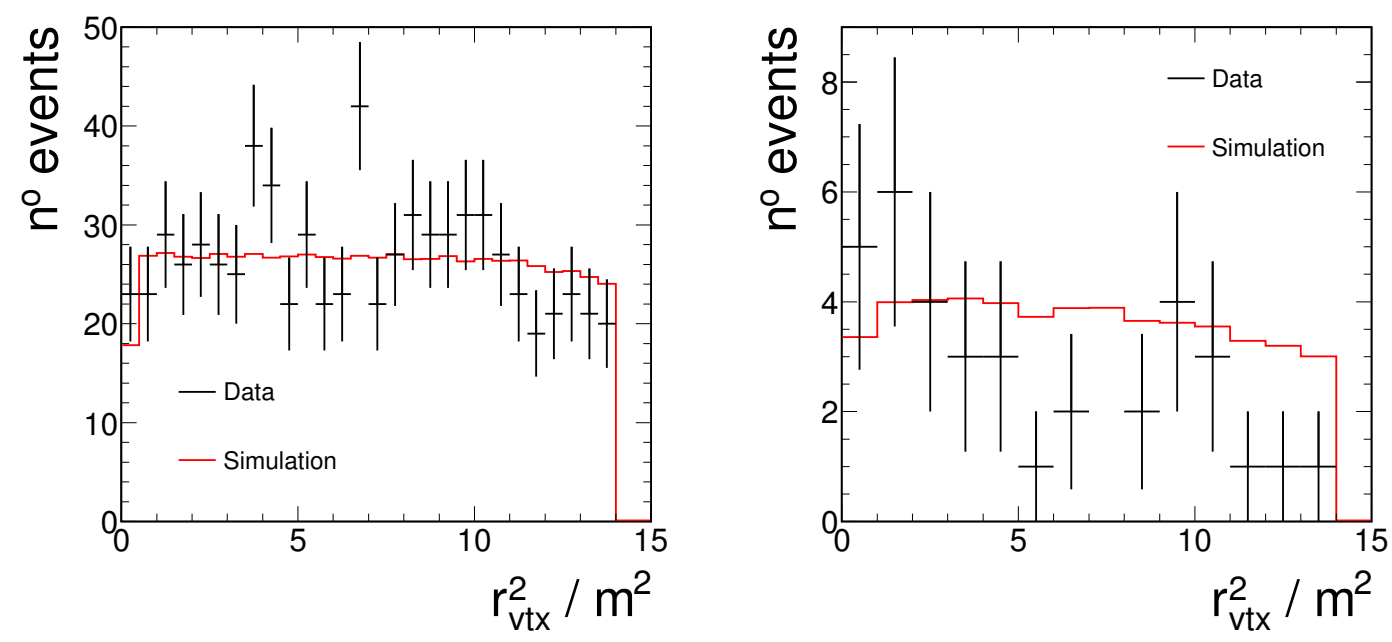

Figure 10.2: The radial position (squared) of the reconstructed track start points for the neutrino events (left) with tracks longer than 35 planes, and the antineutrino events (right), in the data (black) and simulation (red). The simulation has had oscillations applied at $\Delta m_{\mathrm{atm}}^{2}=\Delta \bar{m}_{\mathrm{atm}}^{2}=2.43 \times 10^{-3} \mathrm{eV}^{2}$, $\sin ^{2}\left(2 \theta_{23}\right)=\sin ^{2}\left(2 \bar{\theta}_{23}\right)=1.0$.

region including a large range of mixing angles right up to maximal mixing. MINOS could be observing the corresponding $\bar{\nu}_{\mu}$ disappearance. As discussed in section 2.15 many experiments have ruled out large areas of the allowed LSND parameter space; but none have completely covered it and the most direct cross check, the MiniBooNE collaboration, have run using neutrinos which will not demonstrate this signal if it is truly a result of $\mathcal{C P} \mathcal{T}$ violation (furthermore, the MiniBooNE collaboration observe an as yet unexplained excess in the $\nu_{e}$ spectrum at energies below those thought to be the relevant signal region).

A final possibility is that of a statistical fluctuation. At only $2 \sigma$ this is far from a definitive signal. It could be simple chance that too few events have been observed in this dataset, and as MINOS continues to take data the deficit could disappear. The MINOS collaboration will investigate this possibility more thoroughly before analysing the data, through the use of mock data sets: multiple samples of far detector data simulated with neutrino oscillations, at the same exposure as the real data. This will give a measure of the probability that any one data set could 
fluctuate to give an unexpected result such as that observed in this thesis.

With the current low statistics antineutrino sample, these three possibilities cannot be separated. Too little data exists to conclusively observe any systematic uncertainty should it exist, and the possibility of a statistical fluctuation cannot be eliminated. The puzzle will remain until more data is available.

\subsection{Outlook}

MINOS will continue to take data, at least into 2010. By mid 2009 the statistics presented in this thesis will already have been doubled, reaching an estimated exposure of $7.2 \times 10^{20}$ PoT.

The antineutrino analysis requires much more work, and data, to track down the observed deficit in events as either a statistical fluctuation or systematic mismodelling. Studies such as hand-scanning of the events, particularly those which only just failed the selection cuts, may throw some light on where the events have gone. If these cross checks do not reveal evidence for mismodelling, they will provide invaluable confidence in the validity of the analysis.

Even with $7.2 \times 10^{20}$ PoT of data, only $\sim 100$ charged current $\bar{\nu}_{\mu}$ events would be expected at the far detector without oscillations; and with a peak energy of $\sim 8 \mathrm{GeV}$ only about $10 \%$ of those would disappear with oscillations at $\Delta \bar{m}_{\text {atm }}^{2}=$ $2.43 \times 10^{-3} \mathrm{eV}^{2}$. This still provides very limited sensitivity to oscillations, as shown by the black line in figure 10.3: oscillations can only be observed at less than the $2 \sigma$ level. However, by reversing the current in the NuMI magnetic horns, negative pions can be focused which will decay to give a $\bar{\nu}_{\mu}$ beam. This gives MINOS a unique opportunity to make the first precision measurement of the antineutrino oscillation parameters in the atmospheric regime. At an expected exposure of $2.5 \times 10^{20}$ PoT per year, MINOS will be able to observe oscillations at $7 \sigma$ after two years of running: figure 10.3 also shows the sensitivities for this dedicated 


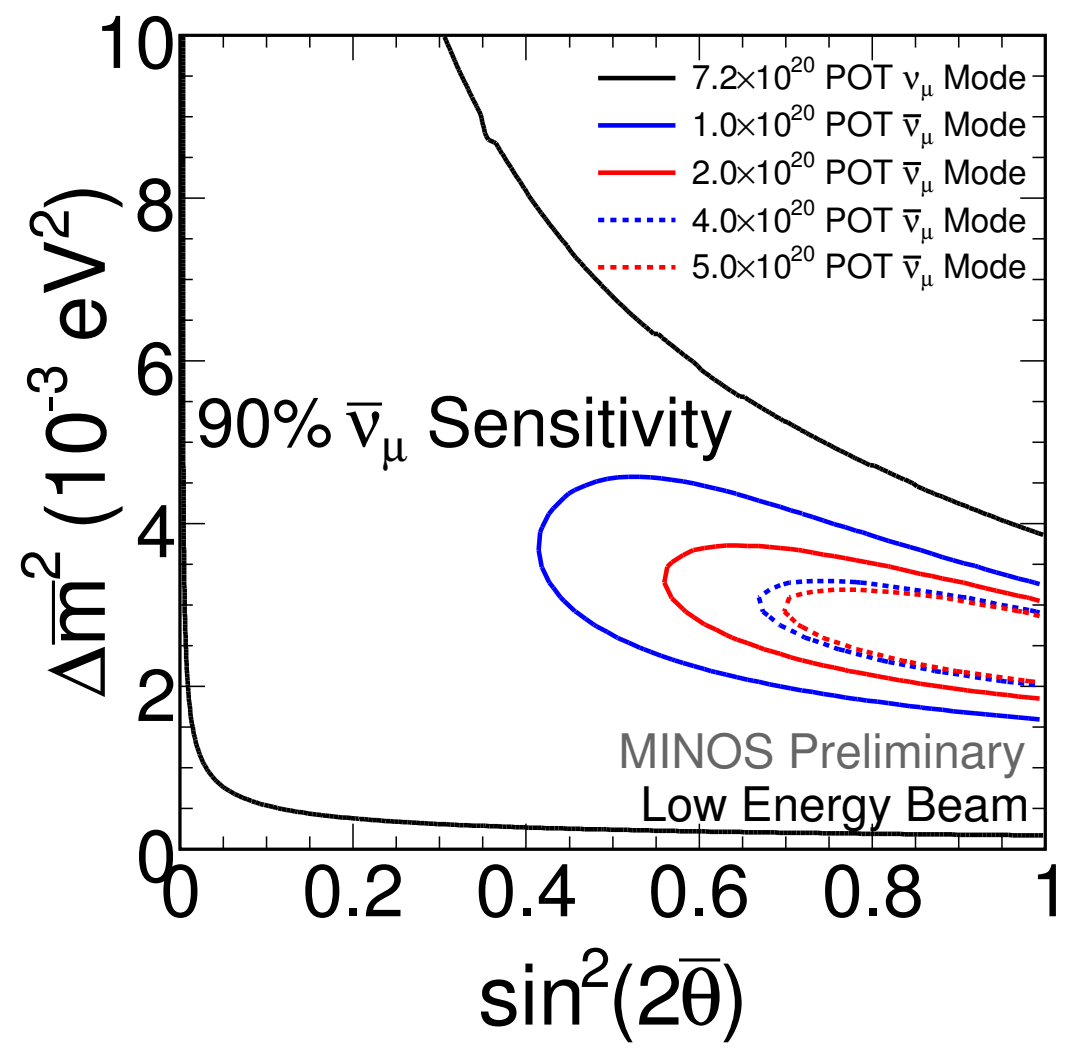

Figure 10.3: The MINOS sensitivity to the antineutrino oscillation parameters with the $\bar{\nu}_{\mu}$ component in the neutrino beam expected by summer 2009 (black line) and with a dedicated $\bar{\nu}_{\mu}$ beam (red and blue lines; a data-taking rate of $2.5 \times 10^{20}$ PoT per year is expected). 
$\bar{\nu}_{\mu}$ running mode. MINOS will be quickly be able to improve by an order of magnitude on the current world limits on $\Delta \bar{m}_{\text {atm }}^{2}$ (shown in figure 2.11).

The next generation long baseline neutrino oscillation experiments, T2K and No $\nu$ a, have no plans to run with antineutrinos for at least five years. This puts MINOS in a position to fill a gap in our experimental knowledge of neutrino oscillations by making a measurement that will stand unrivalled for the foreseeable future. Nevertheless, T2K and Nova both have the capability to run with a $\bar{\nu}_{\mu}$ beam, and if the deficit observed in this thesis does not resolve itself with increased MINOS statistics, this would be a motivation for $\bar{\nu}_{\mu}$ running in these next-generation experiments.

The observed deficit also makes the limits placed on $\nu_{\mu} \rightarrow \bar{\nu}_{\mu}$ transitions dubious: for this to be a meaningful measurement, there must be good confidence in the assumption of $\mathcal{C P} \mathcal{T}$ conservation in the underlying oscillation parameters; this confidence would be given by good agreement between the data and prediction above $10 \mathrm{GeV}$. As statistics increase, and light is shed on any systematic uncertainties causing the deficit, confidence in this transition measurement will increase.

\subsection{Conclusion}

The new measurements presented in this thesis have added to the world's knowledge of neutrino oscillations, and brought to light an unexpected and puzzling observation upon making the first ever direct measurement of $\bar{\nu}_{\mu}$ oscillations in the atmospheric regime. With the MINOS experiment continuing to take data, and the potential of $\bar{\nu}_{\mu}$ running, the collaboration is well placed to continue the work presented in this thesis.

Neutrinos have proved some of the most interesting particles to study in the 50 years since their discovery, the phenomenon of oscillations being one of the most 
exciting and surprising characteristics. The results in this thesis have shown that, although we view the field as one moving into an era of precision measurements, there is still much we do not know; there is still the potential for new surprises and discoveries, and as such the next 50 years promise to be just as exciting as the last. 


\section{Bibliography}

[1] W. Pauli, Letter, addressed to participants of the Tübingen conference on radioactivity 1930, Available from the CERN Document server: http: //cdsweb.cern.ch/record/83282P.

[2] O. Hahn and L. Meitner, Uber die Absorption der $\beta$-Strahlen einiger Radioelemente, Physikalische Zeitschrift 9, 321 (1908).

[3] O. von Baeyer, O. Hahn, and L. Meitner, Nachweis von $\beta$-Strahlen bei Radium D, Physikalische Zeitschrift 12, 378 (1911).

[4] J. Chadwick, Intensitätsverteilung im magnetischen Spektrum der $\beta$ Strahlen von Radium B+C, Verhandlungen der Deutschen Physikalischen Gesellschaft 16, 383 (1914).

[5] C. D. Ellis and W. A. Wooster, The Average Energy of Disintegration of Radium E, Proceedings of the Royal Society of London A117, 109 (1927).

[6] F. Rasetti, On the Raman Effect in Diatomic Gases, Procedings of the National Academy of Sciences of the United States of America 15, 234 (1929).

[7] F. Rasetti, On the Raman Effect in Diatomic Gases. II, Procedings of the National Academy of Sciences of the United States of America 15, 515 (1929). 
[8] W. Heitler and G. Herzberg, Gehorchen die Stickstoffkerne der Boseschen Statistik?, Naturwissenschaften 17, 673 (1929).

[9] J. Chadwick, The Existence of a Neutron, Proceedings of the Royal Society of London A136, 692 (1932).

[10] D. Iwanenko, The Neutron Hypothesis, Nature 129, 798 (1932).

[11] W. Heisenberg, Überden den Bau der Atomkerne. I., Zeitschrift für Physik 77, 1 (1932).

[12] W. Heisenberg, Überden den Bau der Atomkerne. II., Zeitschrift für Physik 78, $156(1932)$.

[13] W. Heisenberg, Überden den Bau der Atomkerne. III., Zeitschrift für Physik 80, 587 (1933).

[14] D. Iwanenko, Interaction of Neutrons and Protons, Nature 133, 981 (1934).

[15] F. Perrin, Possibilité d'émission de particules neutres de masse intrinséque nulle dans les radiactivités $\beta$, Comptes Rendus des Séances de l'Académie des Sciences 197, 1625 (1933).

[16] E. Fermi, Versuch einer Theorie der $\beta$-Strahlen. I., Zeitschrift für Physik 88, 161 (1934).

[17] F. L. Wilson, Fermi's Theory of Beta Decay, American Journal of Physics 36, 1150 (1968).

[18] J. Chadwick and D. Lea, An attempt to detect a neutral particle of small mass, Proceedings of the Cambridge Philosophical Society 30, 59 (1934).

[19] M. Nahmias, An attempt to detect the neutrino, Proceedings of the Cambridge Philosophical Society 31, 99 (1935). 
[20] F. Reines and C. Cowan, Detection of the Free Neutrino, Phys. Rev. 92, 830 (1953).

[21] F. Reines and C. Cowan, A Proposed Experiment to Detect the Free Neutrino, Phys. Rev. 90, 492 (1953).

[22] F. Reines, C. Cowan, F. Harrison, E. Anderson, and F. Hayes, Large Liquid Scintillation Detectors, Phys. Rev. 90, 493 (1953).

[23] F. Reines and C. Cowan, The Neutrino, Nature 178, 446 (1956).

[24] F. Reines, C. Cowan, F. Harrison, A. McGuire, and H. Kruse, Detection of the Free Antineutrino, Phys. Rev. 117, 159 (1960).

[25] R. H. Dalitz, Decay of $\tau$ Mesons of Known Charge, Phys. Rev. 94, 1046 (1954).

[26] D. M. Ritson et al., Characteristics of $K^{+}$-Particles, Phys. Rev. 101, 1085 (1956).

[27] G. Harris, J. Orear, and S. Taylor, Lifetimes of the $\tau^{+}$and $K_{L}^{+}$Mesons, Phys. Rev. 100, 932 (1955).

[28] V. Fitch and R. Motley, Mean Life of $K^{+}$Mesons, Phys. Rev. 101, 496 (1956).

[29] L. W. Alvarez, F. S. Crawford, M. L. Good, and M. L. Stevenson, Lifetimes of $K$ Mesons, Phys. Rev. 101, 503 (1956).

[30] T. D. Lee and C. N. Yang, Question of Parity Conservation in Weak Interactions, Phys. Rev. 104, 254 (1956).

[31] C. S. Wu, E. Ambler, R. W. Hayward, D. D. Hoppes, and R. P. Hudson, Experimental Test of Parity Conservation in Beta Decay, Phys. Rev. 105, 1413 (1957). 
[32] M. Goldhaber, L. Grodzins, and A. W. Sunyar, Helicity of Neutrinos, Phys. Rev. 109, 1015 (1958).

[33] R. P. Feynman and M. Gell-Mann, Theory of the Fermi Interaction, Phys. Rev. 109, 193 (1958).

[34] E. C. G. Sudarshan and R. E. Marshak, Chirality Invariance and the Universal Fermi Interaction, Phys. Rev. 109, 1860 (1958).

[35] E. D. Commins and P. H. Bucksbaum, Weak Interactions of Leptons and Quarks (Cambridge University Press, 1983).

[36] W. Heisenberg, Zur Theorie der "Schauer" in der Höhenstrahlung, Zeitschrift für Physik 101, 533 (1936).

[37] H. Yukawa, On the Interaction of Elementary Particles, Proc. Phys. Math. Soc. Japan 17, 48 (1935).

[38] S. L. Glashow, Partial Symmetries of Weak Interactions, Nucl. Phys. 22, 579 (1961).

[39] A. Salam and J. C. Ward, Electromagnetic and weak interactions, Phys. Lett. 13, 168 (1964).

[40] S. Weinberg, A Model of Leptons, Phys. Rev. Lett. 19, 1264 (1967).

[41] Gargamelle Neutrino Collaboration: F. J. Hasert et al., Search for elastic muon neutrino electron scattering, Phys. Lett. B46, 121 (1973).

[42] Gargamelle Neutrino Collaboration: F. J. Hasert et al., Observation of neutrino-like interactions without muon or electron in the Gargamelle neutrino experiment, Phys. Lett. B46, 138 (1973). 
[43] UA1 Collaboration: G. Arnison et al., Experimental Observation of Isolated Large Transverse Energy Electrons With Associated Missing Energy at $\sqrt{s}=$ 540 GeV, Phys. Lett. B122, 103 (1983).

[44] UA2 Collaboration: R. Banner et al., Observation of Single Isolated Electrons of High Transverse Momentum in Events with Missing Transverse Energy at the CERN $\bar{p} p$ Collider, Phys. Lett. B122, 476 (1983).

[45] UA1 Collaboration: G. Arnison et al., Experimental observation of lepton pairs of invariant mass around $95 \mathrm{GeV} / c^{2}$ at the CERN SPS collider, Phys. Lett. B126, 398 (1983).

[46] UA2 Collaboration: P. Bagnaia et al., Evidence for $Z^{0} \rightarrow e^{+} e^{-}$at the CERN anti-p p collider, Phys. Lett. B129, 130 (1983).

[47] C. D. Anderson and S. H. Neddermeyer, Cloud Chamber Observations of Cosmic Rays at 4300 Meters Elevation and Near Sea-Level, Phys. Rev. 50, $263(1936)$.

[48] S. H. Neddermeyer and C. D. Anderson, Note on the Nature of Cosmic-Ray Particles, Phys. Rev. 51, 884 (1937).

[49] M. Conversi, E. Pancini, and O. Piccioni, On the Disintegration of Negative Mesons, Phys. Rev. 71, 209 (1947).

[50] S. Tomonaga and G. Araki, Effect of the Nuclear Coulomb Field on the Capture of Slow Mesons, Phys. Rev. 58, 90 (1940).

[51] E. Fermi, E. Teller, and V. Weisskopf, The Decay of Negative Mesotrons in Matter, Phys. Rev. 71, 314 (1947).

[52] B. Pontecorvo, Nuclear Capture of Mesons and the Meson Decay, Phys. Rev. 72, 246 (1947). 
[53] O. Klein, Mesons and Nucleons, Nature 161, 897 (1948).

[54] S. Lokanathan and J. Steinberger, Search for Improbable Meson Decays, Phys. Rev. 98, 240 (1955).

[55] G. Danby et al., Observation of High-Energy Neutrino Reactions and the Existence of Two Kinds of Neutrinos, Phys. Rev. Lett. 9, 36 (1962).

[56] M. L. Perl, Reflections on the Discovery of the Tau Lepton 1995, Nobel Lecture; available at http://nobelprize.org/nobel_prizes/physics/ laureates/1995/perl-lecture.pdf.

[57] M. L. Perl et al., Evidence for Anomalous Lepton Production in $e^{+}-e^{-}$ Annihilation, Phys. Rev. Lett. 35, 1489 (1975).

[58] W.-M. Yao et al., Review of Particle Physics, Journal of Physics G: Nuclear and Particle Physics 33, 1 (2006).

[59] DONUT Collaboration: K. Kodama et al., Observation of Tau Neutrino Interactions, Phys. Lett. B504, 218 (2001).

[60] M. Gell-Mann and A. H. Rosenfeld, Hyperons and Heavy Mesons (Systematics and Decay), Annual Review of Nuclear Science 7, 407 (1957).

[61] B. Pontecorvo, Inverse beta processes and nonconservation of lepton charge, Sov. Phys. JETP 7, 172 (1958).

[62] B. Pontecorvo, Neutrino experiments and the question of leptonic-charge conservation, Sov. Phys. JETP 26, 984 (1968).

[63] Z. Maki, M. Nakagawa, and S. Sakata, Remarks on the unified model of elementary particles, Prog. Theor. Phys. 28, 870 (1962).

[64] B. Kayser, On the Quantum Mechanics of Neutrino Oscillation, Phys. Rev. D24, 110 (1981). 
[65] W. Burcham and M. Jobes, Nuclear and Particle Physics (Pearson Education Limited, 1995).

[66] R. Davis, D. S. Harmer, and K. C. Hoffman, Search for neutrinos from the sun, Phys. Rev. Lett. 20, 1205 (1968).

[67] J. N. Bahcall, Neutrino Astrophysics (Cambridge University Press, 1989).

[68] V. Gribov and B. Pontecorvo, Neutrino Astronomy and Lepton Charge, Phys. Lett. B28, 493 (1969).

[69] Kamiokande-II Collaboration: K. S. Hirata et al., Observation of ${ }^{8} \mathrm{~B}$ solar neutrinos in the Kamiokande-II detector, Phys. Rev. Lett. 63, 16 (1989).

[70] J. N. Bahcall and M. H. Pinsonneault, What Do We (Not) Know Theoretically about Solar Neutrino Fluxes?, Phys. Rev. Lett. 92, 121301 (2004).

[71] A. I. Abazov et al., Search for neutrinos from the Sun using the reaction ${ }^{71} \mathrm{Ga}\left(\nu_{e}, e^{-}\right){ }^{71} \mathrm{Ge}$, Phys. Rev. Lett. 67, 3332 (1991).

[72] GALLEX Collaboration: P. Anselmann et al., Solar neutrinos observed by GALLEX at Gran Sasso., Phys. Lett. B285, 376 (1992).

[73] SAGE Collaboration: J. Abdurashitov et al., Measurement of the Solar Neutrino Capture Rate by the Russian-American Gallium Solar Neutrino Experiment During One Half of the 22-Year Cycle of Solar Activity, ZhETP 122, 1 (2002).

[74] GALLEX Collaboration: W. Hampel et al., GALLEX Solar Neutrino Observations: Results for GALLEX IV, Phys. Lett. B447, 127 (1999).

[75] GNO Collaboration: M. Altmann et al., Complete results for five years of GNO solar neutrino observations, Phys. Lett. B616, 174 (2005). 
[76] Y. Fukuda et al., Solar Neutrino Data Covering Solar Cycle 22, Phys. Rev. Lett. 77, 1683 (1996).

[77] K. S. Hirata et al., Real-time, directional measurement of ${ }^{8} \mathrm{~B}$ solar neutrinos in the Kamiokande II detector, Phys. Rev. D 44, 2241 (1991).

[78] Super-Kamiokande Collaboration: S. Fukuda et al., The Super-Kamiokande Detector, Nucl. Instrum. Meth. A501, 418 (2003).

[79] Super-Kamiokande Collaboration: J. Hosaka et al., Solar neutrino measurements in Super-Kamiokande-I, Phys. Rev. D73, 112001 (2006).

[80] SNO Collaboration: B. Aharmim et al., Electron Energy Spectra, Fluxes, and Day-Night Asymmetries of ${ }^{8} \mathrm{~B}$ Solar Neutrinos from the 391-Day Salt Phase SNO Data Set, Phys. Rev. C72, 055502 (2005).

[81] L. Wolfenstein, Neutrino oscillations in matter, Phys. Rev. D17, 2369 (1978).

[82] S. P. Mikheev and A. Y. Smirnov, Resonance enhancement of oscillations in matter and solar neutrino spectroscopy, Sov. J. Nucl. Phys. 42, 913 (1985).

[83] KamLAND Collaboration: S. Abe et al., Precision Measurement of Neutrino Oscillation Parameters with KamLAND, Phys. Rev. Lett. 100, 221803 (2008).

[84] Borexino Collaboration: R. Tartaglia, BOREXINO: the technical description, Nucl. Instrum. Meth. A461, 327 (2001).

[85] Borexino Collaboration: C. Arpesella et al., New Results on Solar Neutrino Fluxes from 192 Days of Borexino Data, Phys. Rev. Lett. 101, 091302 (2008). 
[86] M. Chen, The SNO Liquid Scintillator Project, Nucl. Instrum. Meth. B145, $65(2005)$

[87] F. L. Oberauer and W. Potzel, A large liquid scintillator detector for lowenergy neutrino astronomy, Nucl. Phys. B (Proc. Suppl.) 138, 108 (2005).

[88] G. Barr, S. Robbins, T. Gaisser, and T. Stanev, Uncertainties in Atmospheric Neutrino Fluxes, Phys. Rev. D 74, 094009 (2006).

[89] Kamiokande Collaboration: K. S. Hirata et al., Experimental study of the atmospheric neutrino flux, Phys. Lett. B205, 416 (1988).

[90] NUSEX Collaboration: M. Aglietta et al., Experimental Study of Atmospheric Neutrino Flux in the NUSEX Experiment, Europhys. Lett. 8, 611 (1989).

[91] Fréjus Collaboration: K. Daum et al., Determination of the atmospheric neutrino spectra with the Fréjus detector, Z. Phys. C66, 417 (1995).

[92] IMB Collaboration: J. M. LoSecco et al., Test of Neutrino Oscillations Using Atmospheric Neutrinos, Phys. Rev. Lett. 54, 2299 (1985).

[93] IMB Collaboration: R. Becker-Szendy et al., Electron- and muon-neutrino content of the atmospheric flux, Phys. Rev. D 46, 3720 (1992).

[94] Kamiokande Collaboration: K. Hirata et al., Observation of a small atmospheric $\nu_{\mu} / \nu_{e}$ ratio in Kamiokande, Phys. Lett. 280, 146 (1992).

[95] MACRO Collaboration: S. Ahlen et al., Atmospheric neutrino flux measurement using upgoing muons, Phys. Lett. B357, 481 (1995).

[96] Soudan2 Collaboration: W. Allison et al., Measurement of the atmospheric neutrino flavour composition in Soudan 2, Phys. Lett. B391, 491 (1997). 
[97] Kamiokande Collaboration: Y. Fukuda et al., Atmospheric $\nu_{\mu} / \nu_{e}$ ratio in the multi-GeV energy range, Phys. Lett. B335, 237 (1994).

[98] Super-Kamiokande Collaboration: Y. Fukuda et al., Evidence for oscillation of atmospheric neutrinos, Phys. Rev. Lett. 81, 1562 (1998).

[99] Super-Kamiokande Collaboration: Y. Ashie et al., A measurement of atmospheric neutrino oscillation parameters by Super-Kamiokande I, Phys. Rev. D71, 112005 (2005).

[100] Super-Kamiokande Collaboration: C. Yanagisawa, Recent results from Super-Kamiokande, in Frontiers in Contemporary Physics 2001, Vanderbilt University, Nashville, Tennessee, USA (2001).

[101] Super-Kamiokande Collaboration: Y. Ashie et al., Evidence for an oscillatory signature in atmospheric neutrino oscillations, Phys. Rev. Lett. 93, 101801 (2004).

[102] V. Barger, J. G. Learned, S. Pakvasa, and T. J. Weiler, Neutrino Decay as an Explanation of Atmospheric Neutrino Observations, Phys. Rev. Lett. 82, 2640 (1999).

[103] G. L. Fogli, E. Lisi, A. Marrone, and D. Montanino, Status of Atmospheric Neutrino $\nu_{\mu} \rightarrow \nu_{\tau}$ Oscillations and Decoherence after the first K2K Spectral Data, Phys. Rev. D67, 093006 (2003).

[104] K2K Collaboration: M. H. Ahn et al., Measurement of neutrino oscillation by the K2K experiment, Phys. Rev. D74, 072003 (2006).

[105] The MINOS Collaboration, Preliminary Results from MINOS on $\nu_{\mu}$ Disappearance Based on an Exposure of $2.5 \times 10^{20} 120 \mathrm{GeV}$ Protons on the NuMI Target, (2007), Contributed to the XXIII International Symposium 
on Lepton and Photon Interactions at High Energy, Daegu, Korea, 13-18 August 2007.

[106] NOvA Collaboration: D. S. Ayres et al., NOvA proposal to build a 30kiloton off-axis detector to study neutrino oscillations in the Fermilab NuMI beamline, (2004).

[107] Y. Itow et al., The JHF-Kamioka neutrino project, (2001), hep-ex/0106019.

[108] Super-Kamiokande Collaboration: K. Abe et al., A measurement of atmospheric neutrino flux consistent with tau neutrino appearance, Phys. Rev. Lett. 97, 171801 (2006).

[109] OPERA Collaboration: M. Guler et al., OPERA: An appearance experiment to search for $\nu_{\mu} \leftrightarrow \nu_{\tau}$ oscillations in the CNGS beam. Experimental proposal, CERN-SPSC-2000-028.

[110] OPERA Collaboration: R. Acquafredda et al., First events from the CNGS neutrino beam detected in the OPERA experiment, New J. Phys. 8, 303 (2006).

[111] CHOOZ Collaboration: M. Apollonio et al., Search for neutrino oscillations on a long base-line at the CHOOZ nuclear power station, Eur. Phys. J. C27, $331(2003)$.

[112] T. Schwetz, Global fits to neutrino oscillation data, Phys. Scripta T127, 1 (2006).

[113] Double Chooz Collaboration: F. Ardellier et al., Double Chooz: A search for the neutrino mixing angle $\theta_{13}$, hep-ex/0606025 (2006).

[114] Daya Bay Collaboration: X. Guo et al., A precision measurement of the neutrino mixing angle $\theta_{13}$ using reactor antineutrinos at Daya Bay, hepex/0701029 (2007). 
[115] J. Christensen, J. Cronin, V. Fitch, and R. Turlay, Evidence for the $2 \pi$ Decay of the $K_{2}^{0}$ Meson, Phys. Rev. Lett. 13, 138 (1964).

[116] G. Lüders, Proof of the TCP Theorem, Annals of Physics 2, 1 (1957).

[117] A. Angelopoulos et al., First direct observation of time-reversal noninvariance in the neutral-kaon system, Phys. Lett. B444, 43 (1998).

[118] L. Wolfenstein, Violation of Time Reversal Invariance in $K^{0}$ Decays, Phys. Rev. Lett. 83, 911 (1999).

[119] M. Fidecaro and H.-J. Gerber, The Fundamental Symmetries in the Neutral Kaon System - a Pedagogical Choice, Rept. Prog. Phys. 69, 1713 (2006).

[120] J. Ellis, Theory of the Neutron Electric Dipole Moment, Nuclear Instruments and Methods in Physics Research A284, 33 (1989).

[121] C. A. Baker et al., Improved Experimental Limit on the Electric Dipole Moment of the Neutron, Phys. Rev. Lett. 97, 131801 (2006).

[122] S. Antusch and E. Fernandez-Martinez, Signals of $\mathcal{C P} \mathcal{T}$ Violation and NonLocality in Future Neutrino Oscillation Experiments, Phys. Lett. B665, 190 (2008).

[123] J. D. Chapman, Atmospheric Neutrino Observations in the MINOS Far Detector, PhD thesis University of Cambridge 2007.

[124] A. Strumia and F. Vissani, Implications of Neutrino Data Circa 2005, Nuclear Physics B726, 294 (2005).

[125] LSND Collaboration: A. Aguilar et al., Evidence for neutrino oscillations from the observation of $\bar{\nu}_{e}$ appearance in a $\bar{\nu}_{\mu}$ beam, Phys. Rev. D64, 112007 (2001). 
[126] The MiniBooNE Collaboration: A. A. Aguilar-Arevalo et al., A Search for electron neutrino appearance at the $\Delta m^{2} \sim 1 \mathrm{eV}^{2}$ scale, Phys. Rev. Lett. 98, 231801 (2007).

[127] H. Chen et al., A Proposal for a New Experiment Using the Booster and NuMI Neutrino Beamlines: MicroBooNE, (2007), http:// www-microboone.fnal.gov/Documents/MicroBooNE_10152007.pdf.

[128] Super-Kamiokande Collaboration: S. Fukuda et al., Tau neutrinos favored over sterile neutrinos in atmospheric muon neutrino oscillations, Phys. Rev. Lett. 85, 3999 (2000).

[129] Super-Kamiokande Collaboration: M. Shiozawa, Experimental results on atmospheric neutrinos in Super-Kamiokande-I, (2002), Contributed to Neutrino 2002, Munich, Germany, 25-30 May 2002.

[130] MINOS Collaboration: P. Adamson et al., Search for Active Neutrino Disappearance Using Neutral-Current Interactions in the MINOS Long-Baseline Experiment, Phys. Rev. Lett. 101, 221804 (2008).

[131] H. Klapdor-Kleingrothaus and I. Krivosheina, The Evidence for the Observation of $0 \nu \beta \beta$ Decay: The Identification of $0 \nu \beta \beta$ Events from the Full Spectra, Modern Physics Letters A21, 1547 (2006).

[132] A. Cooper, J. Guy, A. Michette, M. Tyndel, and W. Venus, Limits on Neutrino-Antineutrino Transitions from a Study of High-Energy Neutrino Interactions, Phys. Lett. B112, 97 (1982).

[133] S. Freedman et al., Limits on Neutrino Oscillations from $\bar{\nu}_{e}$ Appearance, Phys. Rev. D47, 811 (1993). 
[134] MINOS Collaboration: P. Adamson et al., First observations of separated atmospheric $\nu_{\mu}$ and $\bar{\nu}_{\mu}$ events in the MINOS detector, Phys. Rev. D73, $072002(2006)$.

[135] MINOS Collaboration: P. Adamson et al., Charge-separated atmospheric neutrino-induced muons in the MINOS far detector, Phys. Rev. D75, 092003 (2007).

[136] MINOS Collaboration: P. Adamson et al., Measurement of the atmospheric muon charge ratio at $\mathrm{TeV}$ energies with MINOS, Phys. Rev. D76, 052003 (2007).

[137] NCAS and MINOS Collaboration: S. Osprey et al., Sudden Stratospheric Warmings seen in MINOS Deep Underground Muon Data, (2009), Accepted for publication by Geophys. Res. Lett..

[138] J. Hylen et al., NuMI Technical Design Handbook, Internal NuMI report (2003).

[139] R. M. Zwaska, Accelerator Systems and Instrumentation for the NuMI Neutrino Beam, PhD thesis University of Texas at Austin, 2005.

[140] MINOS Collaboration: D. G. Michael et al., The Magnetized Steel and Scintillator Calorimeters of the MINOS Experiment, Nucl. Instrum. Meth. A569, 190 (2008).

[141] G. Drake, J. Dawson, and C. Nelson, Overview of the Front End Electronics for the MINOS Near Detector, NuMI-L-628 (1999).

[142] N. Felt et al., MINOS Far Detector Electronics User's Manual, Internal MINOS document (2001).

[143] P. Adamson et al., Calibration of the MINOS Near and Far Detector Readout Systems, To be submitted to Nucl. Instrum. Meth.. 
[144] A. Cabrera, Systematic Comparison of the MINOS Near and Far Detector Readout Systems, DPhil thesis, University of Oxford, 2005.

[145] M. A. Kordosky, Hadronic Interactions in the MINOS Detectors, PhD thesis University of Texas at Austin 2004.

[146] P. L. Vahle, Electromagnetic Interactions in the MINOS Detectors, PhD thesis University of Texas at Austin 2004.

[147] A. Fasso, A. Ferrari, P. R. Sala, and J. Ranft, FLUKA: Status and prospects for hadronic applications, Contributed to the International Conference on Advanced Monte Carlo for Radiation Physics, Particle Transport Simulation and Applications (MC 2000), Lisbon, Portugal, 23-26 October 2000.

[148] GEANT-Detector description and simulation tool, CERN Program Library, Long Writeup, W5013, http://wwwasd.web.cern.ch/wwwasd/ geant/index.html.

[149] H. Gallagher, The NEUGEN neutrino event generator, Nucl. Phys. Proc. Suppl. 112, 188 (2002).

[150] C. Zeitnitz and T. A. Gabriel, The GEANT - CALOR interface and benchmark calculations of ZEUS test calorimeters, Nucl. Instrum. Meth. A349, 106 (1994).

[151] C. J. Llewellyn Smith, Neutrino Reactions at Accelerator Energies, Phys. Rep. 3, 261 (1972).

[152] M. E. Dorman, Cross Section Measurements for Quasi-Elastic NeutrinoNucleus Scattering with the MINOS Near Detector, $\mathrm{PhD}$ thesis University College London 2008.

[153] D. Rein and L. M. Sehgal, Neutrino-Excitation of Baryon Resonances and Single Pion Production, Ann. Phys. 133, 79 (1981). 
[154] R. Devenish and A. Cooper-Sarkar, Deep Inelastic Scattering (Oxford University Press, 2004).

[155] R. E. Kalman, Trans. ASME, J. Bas. Eng. 82D, 35 (1960).

[156] R. Fruhwirth, Application of Kalman Filtering to Track and Vertex Fitting, Nucl. Instrum. Meth. A262, 4442 (1987).

[157] J. S. Marshall, A Study of Muon Neutrino Disappearance with the MINOS Detectors and the NuMI Neutrino Beam, PhD thesis University of Cambridge 2008 .

[158] MINOS Collaboration: D. G. Michael et al., Observation of muon neutrino disappearance with the MINOS detectors and the NuMI neutrino beam, Phys. Rev. Lett. 97, 191801 (2006).

[159] MINOS Collaboration: P. Adamson et al., Study of Muon Neutrino Disappearance using the Fermilab Main Injector Neutrino Beam, Phys. Rev. D77, 072002 (2008).

[160] J. Hartnell, Measurement of the Calorimetric Energy Scale in MINOS, DPhil thesis, University of Oxford, 2005.

[161] N. Tagg et al., Performance of Hamamatsu 64-anode photomultipliers for use with wavelength-shifting optical fibres, Nucl. Instrum. Meth. A539, 668 (2005).

[162] K. Lang et al., Characterization of 1600 Hamamatsu 16-anode photomultipliers for the MINOS Far detector, Nucl. Instrum. Meth. A545, 852 (2005).

[163] P. Symes, Preliminary Measurement of Neutrino Oscillation Parameters By NuMI/MINOS And Calibration Studies For Improving This Measurement, DPhil thesis, University of Sussex, 2005. 
[164] H. Bethe, Zur Theorie des Durchgangs schneller Korpuskularstrahlen durch Materie, Annalen der Physik (397), 325 (1930).

[165] F. Bloch, Zur Bremsung rasch bewegter Teilchen beim Durchgang durch Materie, Annalen der Physik (408), 285 (1933).

[166] C. Andreopoulos, P. Stamoulis, and G. Tzanakos, Temperature Effects on M16 PMTs, NuMI-Note-SCINT-988 (2002).

[167] P. Miyagawa, A. Weber, and C. Perry, Measurements of Gain and Noise Properties of Hamamatsu M64 PMT's, NuMI-L-590 (2000).

[168] Hamamatsu Photonics K.K., Photomultiplier Tubes: Basics and Applications (Hamamatsu Photonics K.K., 1999), Second edition.

[169] G. D. Barr, The Separation of Signals and Background in a Nucleon Decay Experiment, DPhil thesis, University of Oxford, 1987.

[170] A. B. P. Sousa, Aspectos da Detecção de Raios Cósmicos em A.M.S., Master's thesis, Instituto Superior Técnico 1987.

[171] C. Amsler et al., Review of Particle Physics, Phys. Lett. B667, 1 (2008).

[172] G. Tinti, private communication 2007.

[173] R. Brun and F. Rademakers, ROOT: An object oriented data analysis framework, Nucl. Instrum. Meth. A389, 81 (1997).

[174] R. Ospanov, A Measurement of Muon Neutrino Disappearance with the MINOS Detectors and NuMI Beam, PhD thesis University of Texas at Austin 2008.

[175] M. Szleper and A. Para, Neutrino Spectrum at the Far Detector; Systematic Erors, NuMI-B-781 (2001). 
[176] P. Renton, Electroweak Interactions (Cambridge University Press, 1990).

[177] F. James and M. Roos, MINUIT - A System for function minimization and analysis of the parameter errors and correlations, Comput. Phys. Commun. 10, $343(1975)$.

[178] M. Kordosky, H. Gallagher, and S. Dytman, Shower Energy Scale Uncertainty for the Run I+II CC Analysis, MINOS-doc-4287 (2008).

[179] R. Nichol, private communication 2008.

[180] R. Hatcher et al., Range/Energy Task Force Position Paper, MINOS-doc$3134(2007)$.

[181] A. I. Himmel, M. Ishitsuka, and D. Jaffe, Systematic Error on Antineutrinos from the Decay Pipe, MINOS-doc-4584 (2008).

[182] C. Andreopoulos et al., Updated Cross Section Model Uncertainties for the Charged Current Analysis, MINOS-doc-2989 (2007).

[183] J. Marshall, NC Background Update, MINOS-doc-3313 (2007).

[184] J. Thomas, Comparison of CalDet muons and simulation, MINOS-doc-3993 (2008).

[185] V. Bocean, Status Report on the Geodetic and Alignment Efforts for the NuMI Project at Fermilab, (2004), Proceedings of the Eighth International Workshop on Accelerator Alignment, Geneva, Switzerland.

[186] R. Ospanov and K. Lang, Alignment of the Near Detector Scintillator Modules using Cosmic Ray Muons, FERMILAB-TM-2411-E (2005).

[187] R. Ospanov and K. Lang, Alignment of the MINOS Far Detector, FERMILAB-TM-2410-E (2004). 
[188] MINOS Collaboration: P. Adamson et al., Measurement of Neutrino Oscillations with the MINOS Detectors in the NuMI Beam, Phys. Rev. Lett. 101, 131802 (2008). 